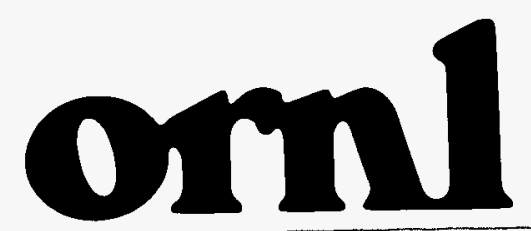

OAK RIDGE NATIONAL LABORATORY

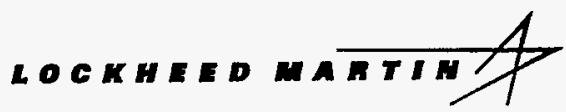

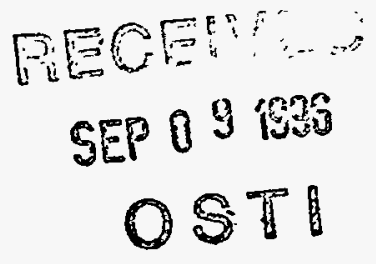

ORNL-6895

\title{
ORTEP-III: Oak Ridge Thermal Ellipsoid Plot Program for Crystal Structure Illustrations
}

Michael N. Burnett

Carroll K. Johnson

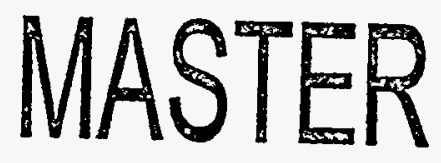


This report has been reproduced directly from the best available copy.

Avallable to DOE and DOE contractors from the Office of Scientific and Techntcal Information, P.O. Box 62, Oak Ridge, TN 37831; prices available from (423) 576-8401, FTS 626-8401.

Available to the public from the National Technical Information Service, U.S. Department of Commerce, 5285 Port Royal Rd., Springfield, VA 22161.

This report was prepared as an account of work sponsored by an agency of the United States Government. Neither the United States Government nor any agency thereof, nor any of their employees, makes any warranty, express or implied, or assumes any legal liability or responsibility for the accuracy, completeness, or usefulness of any information, apparatus, product, or process disclosed, or represents that its use would not infringe privately owned rights. Reference herein to any specific commercial product, process, or service by trade name, trademark, manufacturer, or otherwise, does not necessarily constitute or imply its endorsement, recommendation, or favoring by the United States Government or any agency thereof. The views and opinions of authors expressed herein do not necessarily state or refiect those of the United States Government or any agency thereof. 
Chemical and Analytical Sciences Division

\title{
ORTEP-II: OAK RIDGE THERMAL ELLIPSOI PLOT PROGRAM FOR CRYSTAL STRUCTURE ILLUSTRATIONS
}

Michael N. Burnett

Carroll K. Johnson

Date Published: July 1996

Research sponsored by the

Laboratory Directed Research and Development Program

\author{
Prepared by the \\ OAK RIDGE NATIONAL LABORATORY \\ Oak Ridge, Tennessee 37831-6285 \\ managed by \\ LOCKHEED MARTIN ENERGY RESEARCH CORP. \\ for the \\ U.S. DEPARTMENT OF ENERGY \\ under contract DE-AC05-960R22464
}




\section{DISCLAIMER}

Portions of this document may be illegible in electronic image products. Images are produced from the best available original document. 


\section{CONTENTS}

ACKNOWLEDGEMENTS.$\ldots \ldots \ldots \ldots \ldots \ldots \ldots \ldots \ldots \ldots \ldots \ldots \ldots \ldots \ldots \ldots$ vii

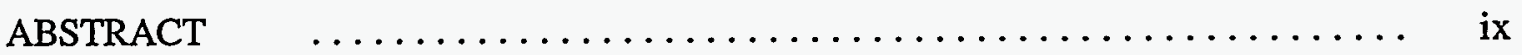

1. INTRODUCTION $\ldots \ldots \ldots \ldots \ldots \ldots \ldots \ldots \ldots \ldots \ldots \ldots \ldots \ldots \ldots \ldots$

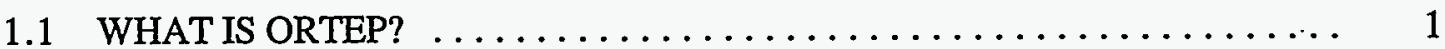

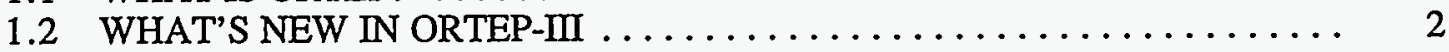

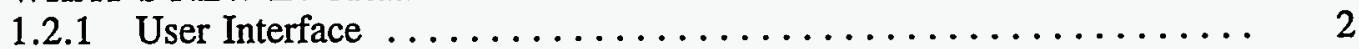

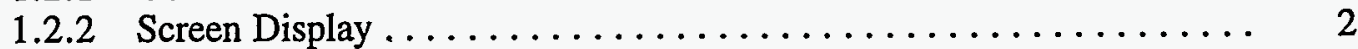

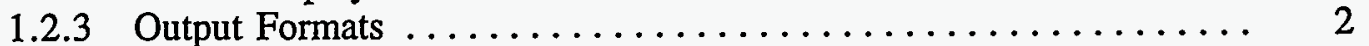

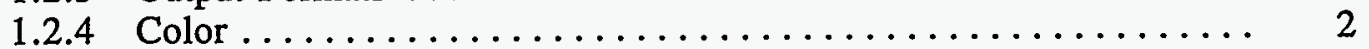

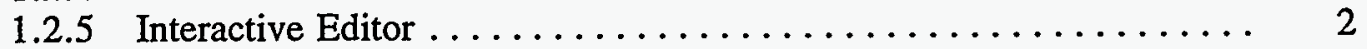

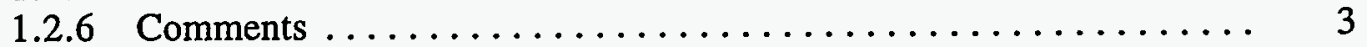

1.2.7 Alternate Formats for Atomic Parameters . . . . . . . . . . . 3

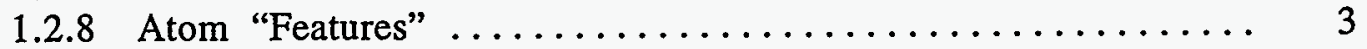

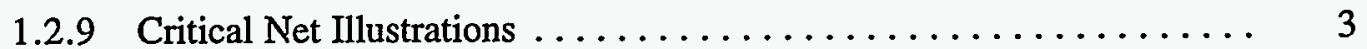

1.2.10 Symmetry Operator Format ................... 3

1.2.11 Miscellaneous Changes in ORTEP-III .............. 4

1.3 REPORT ORGANIZATION $\ldots \ldots \ldots \ldots \ldots \ldots \ldots \ldots \ldots \ldots \ldots \ldots \ldots \ldots \ldots \ldots$

2. PROGRAMMING ORTEP $\ldots \ldots \ldots \ldots \ldots \ldots \ldots \ldots \ldots \ldots \ldots \ldots \ldots \ldots \ldots \ldots \ldots \ldots$

2.1 GENERAL PRINCIPLES . . . . . . . . . . . . . . . $5 \ldots \ldots$.

2.2 PROGRAMMING A NONSTEREOSCOPIC ILLUSTRATION FOR ORTEP . $\quad 10$

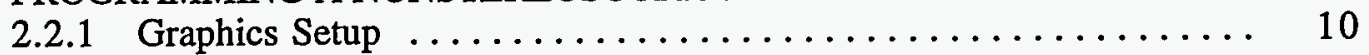

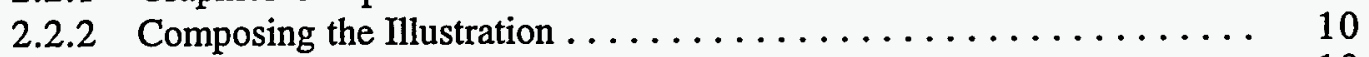

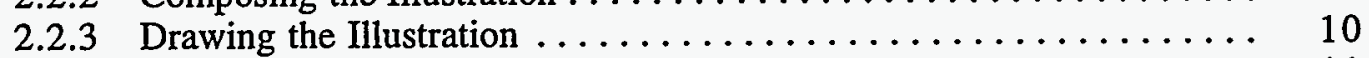

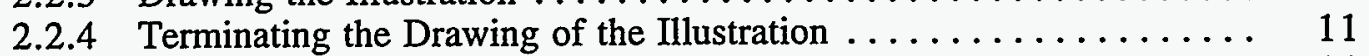

2.3 PROGRAMMING A STEREOSCOPIC ILLUSTRATION FOR ORTEP . . . . 11

2.3.1 Stereoscopic Rotations ....................... 11

2.3.3 Repeating a Sequence of Operations ................. 12

2.4 DRAWING THE CUBANE STRUCTURE: AN EXAMPLE ........... 12

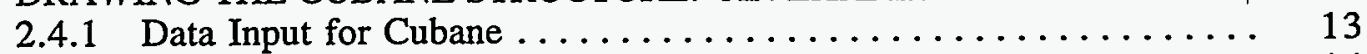

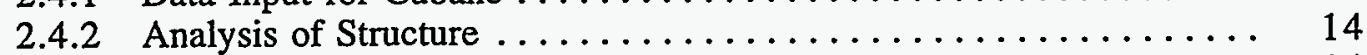

2.4.3 Programming the Cubane Illustration $\ldots \ldots \ldots \ldots \ldots \ldots \ldots \ldots . \ldots \ldots \ldots$

2.4.4 Illustration of the Example $\ldots \ldots \ldots \ldots \ldots \ldots \ldots \ldots \ldots \ldots \ldots \ldots$

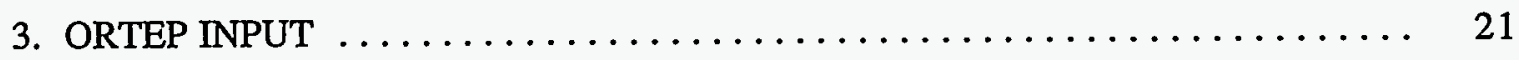

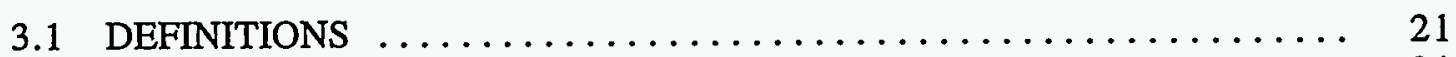

3.1.1 Atom Designator Code (ADC) and Addressable Point ......... 21

3.1.2 Vector Designator Code (VDC) .................. 21

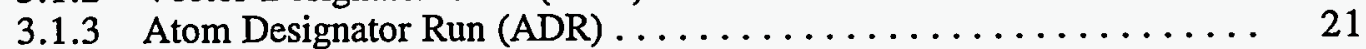

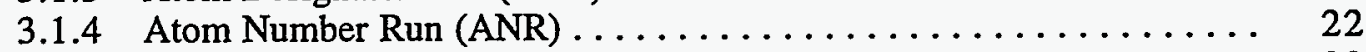

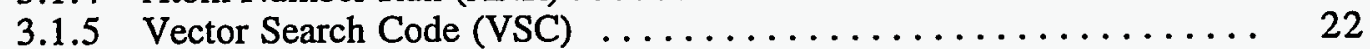

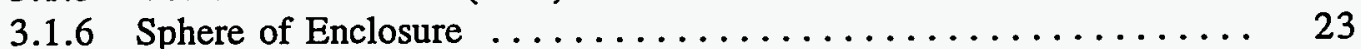

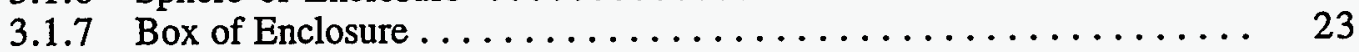


3.1.8 Reference, Working, and Standard Cartesian Coordinate Systems ... 23

3.1.9 Prime Parameters and Primer Constants ................ 23

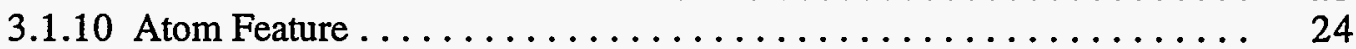

3.1 .11 Feature Number Run (FNR) . . . . . . . . . . . . . . . . 24

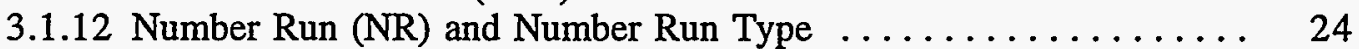

3.2 CRYSTAL STRUCTURE DATA INPUT $\ldots \ldots \ldots \ldots \ldots \ldots \ldots \ldots \ldots . \ldots \ldots$

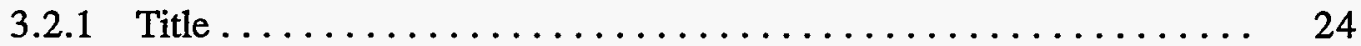

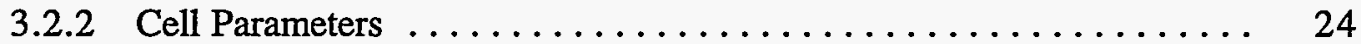

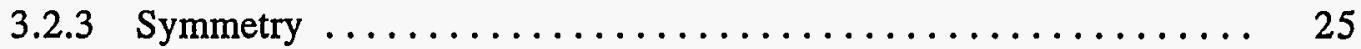

3.2 .4 Atom Parameters . . . . . . . . . . 27

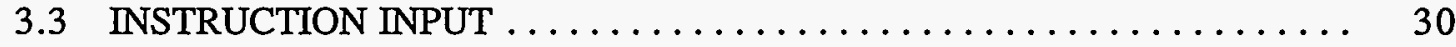

3.3.1 Instruction Format .......................... 30

3.3.2 Structure Analysis Instructions (100 Series) . . . . . . . . . 32

3.3.3 Plotter Control Instructions (200 Series) ............... 34

3.3.4 Drawing Parameter Instructions (300 Series) $\ldots \ldots \ldots \ldots \ldots \ldots . \ldots . \ldots . \ldots . \ldots$

3.3.5 ATOMS Array Instructions (400 Series) $\ldots \ldots \ldots \ldots \ldots \ldots \ldots . \ldots \ldots$

3.3.6 Orienting Instructions $(500$ Series) $\ldots \ldots \ldots \ldots \ldots \ldots \ldots \ldots . \ldots \ldots . \ldots \ldots$

3.3.7 Positioning and Scaling Instructions (600 Series) . . . . . . . . . . 44

3.3.8 Atom Plotting Instructions (700 Series) $\ldots \ldots \ldots \ldots \ldots \ldots \ldots . \ldots \ldots$

3.3.9 Bond Plotting Instructions ( 800 Series) $\ldots \ldots \ldots \ldots \ldots \ldots \ldots \ldots$

3.3.10 Label Plotting Instructions (900 Series) .............. 53

3.3.11 Save Sequence Instructions $(1100$ Series $) \ldots \ldots \ldots \ldots \ldots \ldots \ldots$

33.12 Overlap Correction Instructions $(1001,821,822) \ldots \ldots \ldots \ldots \ldots$

3.3.13 Termination Instructions (Negative Series) . . . . . . . . . 58

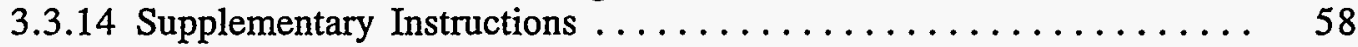

3.4 LIST OF FAULT INDICATORS $\ldots \ldots \ldots \ldots \ldots \ldots \ldots \ldots \ldots \ldots \ldots \ldots$

4. USING ORTEP-III $\ldots \ldots \ldots \ldots \ldots \ldots \ldots \ldots \ldots \ldots \ldots \ldots \ldots \ldots \ldots \ldots \ldots$

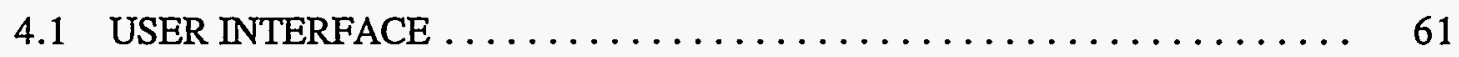

4.2 SCREEN DISPLAY OF THE ORTEP ILLUSTRATION $\ldots \ldots \ldots \ldots \ldots \ldots .62$

4.3 PLOTTING THE ORTEP ILLUSTRATIONS $\ldots \ldots \ldots \ldots \ldots \ldots \ldots \ldots .62$

4.4 INTERACTIVE EDITOR $\ldots \ldots \ldots \ldots \ldots \ldots \ldots \ldots \ldots \ldots \ldots \ldots \ldots \ldots \ldots \ldots$

4.5 ALTERNATE FORMATS FOR ATOMIC PARAMETERS $\ldots \ldots \ldots \ldots \ldots 64$

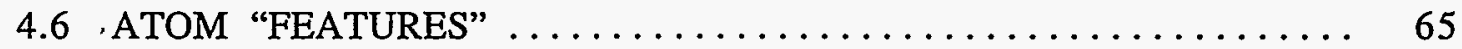

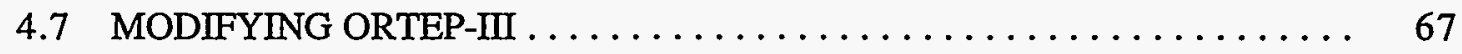

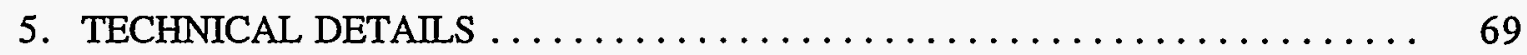

5.1 HOW ORTEP DRAWS ELLIPSOIDS $\ldots \ldots \ldots \ldots \ldots \ldots \ldots \ldots \ldots$

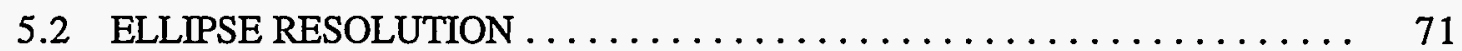

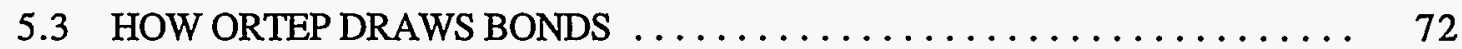

5.4 OPTIMAL PARAMETERS FOR STEREOSCOPIC DRAWINGS $\ldots \ldots \ldots \ldots \quad 74$

6. MATHEMATICS OF THERMAL-MOTION PROBABILITY ELLIPSOIDS $\ldots \ldots \quad 79$

6.1 PROBABILITY DENSITY FUNCTION OF A

TRIVARIATE NORMAL DISTRIBUTION . . . . . . . . . . . . . . . 79

6.2 EQUIPROBABILITY ELLIPSOIDS $\ldots \ldots \ldots \ldots \ldots \ldots \ldots \ldots \ldots \ldots \ldots \ldots . \quad 80$

6.3 CHARACTERISTIC FUNCTION OF A

TRIVARIATE NORMAL DISTRIBUTION $\ldots \ldots \ldots \ldots \ldots \ldots \ldots \ldots . \quad 80$

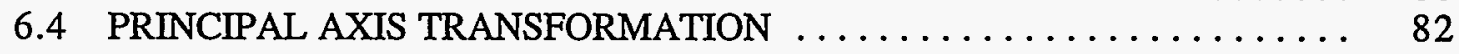




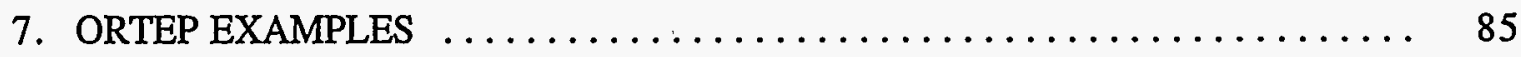

7.1 CELL PACKING - 5-HYDROXY-5-PHENYLNORBORNANONE $\ldots \ldots \ldots \quad 85$

7.2 HELICAL STRUCTURE - POLY-L-ALANINE $\ldots \ldots \ldots \ldots \ldots \ldots \ldots . \quad 89$

7.3 COORDINATION POLYHEDRA - POTASSIUM PERXENATE

NONAHYDRATE $\ldots \ldots \ldots \ldots \ldots \ldots \ldots \ldots \ldots \ldots \ldots \ldots \ldots \ldots \ldots \ldots, 93$

7.4 ATOM FEATURES - LYSOSOME MUTANT POLYPEPTIDE . . . . . . . 96

7.5 CRITICAL NET - SODIUM CHLORIDE $\ldots \ldots \ldots \ldots \ldots \ldots \ldots \ldots \ldots \ldots$

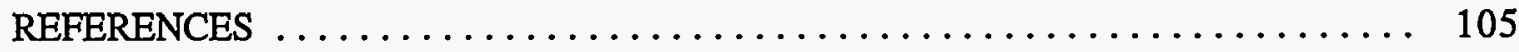

APPENDIX A - ORTEP-III SUBPROGRAMS $\ldots \ldots \ldots \ldots \ldots \ldots \ldots \ldots \ldots 107$

APPENDIX B — GLOSSARY OF VARIABLES IN ORTEP-III COMMONS $\ldots \ldots \ldots 113$

APPENDIX C — ORTEP-II FORTRAN SOURCE CODE LISTING $\ldots \ldots \ldots \ldots \quad 119$ 



\section{ACKNOWLEDGEMENTS}

From previous versions of this report: ${ }^{1,2}$

We are particularly indebted to our colleagues, Drs. H. A. Levy, W. R. Busing, G. M. Brown, and R. D. Ellison for many helpful discussions and to R. A. Hollister, a summer participant with the ORNL Mathematics Division, who helped plan and code several parts of the initial 1965 release of the program. The initial draft version of ORTEP was written as a subroutine for the Busing, Martin, and Levy Function and Error Program, OR FFE; and many of the concepts and several of the subroutines of OR FFE are incorporated into the present program. Several parts of EIGEN were taken from a program written by R. E. Funderlic and B. Franz from the Central Data Processing group. Subroutine AXEQB was adapted from a subroutine obtained from the Oak Ridge Central Data Processing Library.

Additional acknowledgements:

We wish to thank Oak Ridge National Laboratory for providing computer resources for our ORTEP-III (and related crystallographic topology) World Wide Web pages at

http://www.ornl.gov/ortep/ortep.html

We especially thank Dr. Martin Kroeker for setting up a European World Wide Web site to mirror Oak Ridge's ORTEP-III web site at

http://tutor.oc.chemie.th-darmstadt.de/Ortep3/ortep.html

We thank all the people who have used ORTEP over the years and who have modified the program and made it available for others to use, including those listed at

http://www.ornl.gov/ortep/kin.html

A number of people assisted with the testing of ORTEP-III before the program was released to the public. It is impossible to list everyone here, but we appreciate all their efforts. We particularly thank: Dr. John Bollinger, Karl A. Byriel, Bjorn Dalhus, Dr. Bill Harrison, Dr. John C. Huffman, Prof. Gerald G. Johnson, Jr., Dr. Martin Kroeker, Dr. Anthony Linden, Dr. Wolfgang Poll, Dr. James V. Silverton, and Dr. Beverly R. Vincent.

We also thank Dr. Tim Pearson at the California Institute of Technology for the free graphics package PGPLOT, which enabled us to create one version of ORTEP-III that would produce screen graphics on a wide range of computer hardware. Thanks to Christian T. Dum and John S. Salmento for assistance with their ports of PGPLOT for DOS and Macintosh personal computers, respectively.

Thanks go to Kate Crennell for producing an executable version of ORTEP-III for Acorn microcomputers running the RISC OS operating system, which can be found at

http://micros.hensa.ac.uk/micros/arch-riscos.html

and again to Dr. Kroeker for making a PC LINUX version available at

ftp://tutor.oc.chemie.th-darmstadt.de/pub/ortep/linux/ 


\begin{abstract}
This report describes a computer program for drawing crystal structure illustrations. Ball-and-stick type illustrations of a quality suitable for publication are produced with either spheres or thermal-motion probability ellipsoids on the atomic sites. The program can also produce stereoscopic pairs of illustrations which aid in the visualization of complex packing arrangements of atoms and thermal motion patterns. Interatomic distances, bond angles, and principal axes of thermal motion are also calculated to aid the structural study.
\end{abstract}




\section{INTRODUCTION}

\subsection{WHAT IS ORTEP?}

Appropriate illustrations are essential in any manuscript dealing with crystallographic structures. An often quoted expression might justifiably be paraphrased to read that a wellplanned figure is worth a thousand numbers. With the information explosion in the scientific literature, the author of a structure paper is obligated to provide the reader with "crystal clear" illustrations.

The Oak Ridge Thermal Ellipsoid Plot (ORTEP) program is a tool for drawing certain types of crystal structure illustrations. The program and the precision obtainable through machine plotting make feasible the production of detailed stereoscopic illustrations that are impractical to draw by conventional drafting methods. Several types of illustrations may be drawn with ORTEP. For a standard thermal motion drawing, an ellipsoid positioned on an atomic site represents a 3-D Gaussian probability density function showing the averaged atomic displacement as derived through the anisotropic temperature factor parameters for that atom. In critical net drawings, a very elongated or flattened ellipsoid of revolution, not positioned on an atomic center, represents the orientation of a saddle-point type critical point of the global density function. For simpler drawings of a crystal structure, all atoms are represented as spheres with sphere radii, or some other graphical variable, used to depict the chemical type of the atom. ORTEP cannot make van der Waal's type drawings, which require overlapping spheres or ellipsoids.

Since its inception, four major goals have driven the development of ORTEP. These are listed here in decreasing order of their assigned importance. (1) The program must produce high quality illustrations, including stereoscopic pairs of thermal-motion figures, as free as possible of visually distracting approximations. (2) The program must be general both with respect to the types of illustrations it can draw and the types of computing and plotting equipment that it can utilize. (3) The program must be easy to use, require a minimum of input, and be easy to modify. (4) The computation time should be minimized. Since generality is placed higher in this goal list than ease of use, the program originally designed in the 1960s lacks several of the user-friendly attributes of the 1990 s. However, the program seems to have survived the test of time better than some of its more user-friendly competitors. There were over 1000 citations of the $1965^{1}$ and $1976^{2}$ versions of the program in the 1995 Science Citation Index. ${ }^{3}$ Since ORTEP has become widely used, we decided not to make changes that would render existing ORTEP input data sets inoperative.

ORTEP-III is written in device-independent FORTRAN, and the code should compile and run on any computer system that has a FORTRAN compiler. Compiled versions of ORTEP-

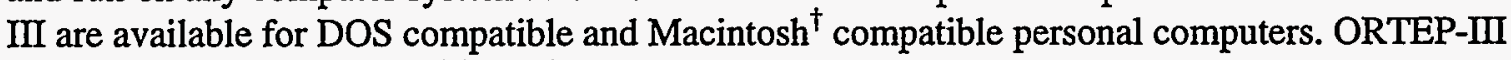
is available on the World Wide Web at

http://www.ornl.gov/ortep/ortep.html

or via anonymous ftp at $\mathrm{ftp}: / / \mathrm{ftp}$. ornl.gov/pub/ortep. Questions, comments, problems, suggestions, etc. may be sent via electronic mail to ortep@ornl.gov or via regular mail to either author of this report at P.O. Box 2008, Oak Ridge, TN 37831-6197, USA.

†Macintosh is a registered trademark of Apple Computer, Inc. 


\subsection{WHAT'S NEW IN ORTEP-III}

For compatibility with existing ORTEP data sets, ORTEP-III retains ALL the functionality of OR TEP, ${ }^{1}$ released in 1965 , and OR TEP-II, ${ }^{2}$ released in 1976. A brief description of the new capabilities in ORTEP-III is provided here. See the indicated sections of this report for more detailed information.

\subsubsection{User Interface}

ORTEP-III is a semi-interactive program that requests certain information from the user while the program executes. A default value for each item requested is provided in square brackets, and it will be used if the user simply hits the RETURN key (on some keyboards, the name of this key is ENTER). On systems that distinguish upper and lower case, care must be taken when entering file names to provide the correct case of the letters. (See 4.1.)

\subsubsection{Screen Display}

The screen drawing subroutines available in ORTEP-III use PGPLOT. PGPLOT is a free graphics library developed by T. J. Pearson at the California Institute of Technology. The package is written in FORTRAN and operates on a variety of platforms. Information about PGPLOT can be found on the World Wide Web at http://astro.caltech.edu/ tjp/pgplot or via e-mail to tjp@astro.caltech.edu. PGPLOT is not required to run ORTEP-III. The compiled versions of ORTEP-III for personal computers include the screen drawing capability. (See 4.2.)

\subsubsection{Output Formats}

Illustrations generated by ORTEP-III can be saved as Encapsulated Postscript ${ }^{\mathrm{TM}}$ or Hewlett-Packard Graphics Language (HPGL/2) ${ }^{\mathrm{TM}}$ files. ${ }^{\dagger}$ The files may be printed directly on a wide variety of printers and plotters or may be imported into a number of computer programs that accept these formats. (See 4.3.)

\subsubsection{Color}

By default, ORTEP-III plots its illustrations in black on a white background. The new 204 instruction allows color to be added to the illustrations if the output device supports color. Once a color is set, it remains in effect until another 204 instruction changes the color. A 204 with no parameter (or a "0") returns the plotting color to black (or pen \#1). The screen and Postscript drivers built into ORTEP-III define color value 2 as red, 3 as green, 4 as blue, 5 as cyan, 6 as magenta, and 7 as yellow. (See 3.3.3.)

\subsubsection{Interactive Editor}

ORTEP-III provides a simple line editor for editing the input file without exiting the program. When the editor is invoked, the instruction set from the input file is displayed on the screen with line numbers (\#) along with the editor commands shown below. (See 4.4.)

†Encapsulated Postscript is a registered trademark of Adobe Systems, Inc. HewlettPackard Graphics Language Version 2 (HPGL/2) is a registered trademark of Hewlett-Packard Corporation. 


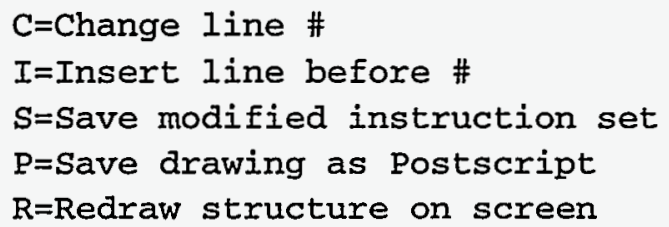

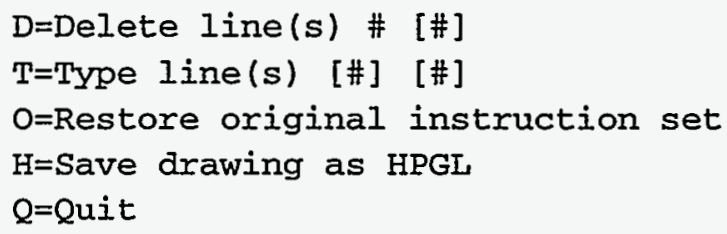

\subsubsection{Comments}

In previous versions of ORTEP, Format No. 3 trailer cards (see 3.3.1) were the only means of placing comments in the input file. This method still works in ORTEP-III, but a new method also exists. Among the ORTEP instructions, any line beginning with \# is treated as a comment and is totally ignored by the program. Such comments can only go in the instruction portion of the input data and only at places where a new instruction could begin, i.e., comments cannot go between instructions and their trailer (continuation) cards. CAUTION: These comment lines are not printed in the ORTEP output file, and they are lost if the input file is edited with ORTEP-III's line editor.

\subsubsection{Alternate Formats for Atomic Parameters}

ORTEP-III provides a way to input atom parameters that are available in a "nonstandard" format. If the sentinel value (column 1) on the last symmetry card (see 3.2.3) in the ORTEP input file is " 2 " instead of " 1 ", the program asks the user for the name of a file containing the atom parameters and branches to subroutine READIN to read the information. This subroutine may be recoded to read any desired format. (See 4.5.)

\subsubsection{Atom "Features"}

In earlier versions of ORTEP, atoms could be referenced only by their numeric positions in the input file; and atom number runs (ANR) (see 3.1.4) were used to select groups of atoms to be treated in the same manner. ORTEP-III"allows two optional attributes called "features" to be included with each atom, and feature number runs (FNR) can be used to select groups of atoms having particular features. To handle features, a new parameter, number run type, has been added to the 100 series, 400 series, 505, 506, 700 series, 800 series, and 1001 instructions. Features should prove especially useful for polymeric materials such as proteins or nucleic acids and for critical net illustrations. (See 4.6.)

\subsubsection{Critical Net Illustrations}

ORTEP-III can produce critical net illustrations that depict some canonical topological characteristics of the global ensemble of overlapping atomic-thermal-motion Gaussian density functions in a crystal. (See 7.5.)

\subsubsection{Symmetry Operator Format}

The symmetry operators in the ORTEP input file (see 3.2.3) may now be provided in a free format using the xyz coordinate triplet notation found in the International Tables for Crystallography. ${ }^{4}$ ORTEP is informed that this style for the symmetry operators is being used by having a " 1 " in column 1 of the cell parameter card (see 3.2.2). (A " 0 " or blank in that position indicates the old style is being used.) 
Symmetry cards using this style do not have a specific format with the following two exceptions: (1) the symmetry information on each card must not go beyond column 72 , and (2) column 1 must be blank on all symmetry cards other than the last one in the set, which must have a non-zero value in column 1 . Below is an example set of symmetry cards to illustrate the flexibility of this style.

$$
\begin{aligned}
& X, Y, Z \\
& X \quad-Y \quad Z+1 / 2 \\
& X+0.5, \quad .5+Y, \quad Z \\
& X+1 / 2,-Y+1 / 2,1 / 2+Z
\end{aligned}
$$

Letters may be either upper or lower case. Commas or spaces may be used to separate the components of the triplet. The three components may not have spaces within them. Decimal fractions may be used with or without an initial 0 . Fractions may precede or follow the letters.

\subsubsection{Miscellaneous Changes in ORTEP-III}

- No longer provides a choice of centered symbols.

- Adds a parameter LOGC to the 100 and 400 series instructions to control the logic used (union or intersection) when multiple screening conditions are applied to the atoms. (See 3.1.5.)

- Makes the parameters on the Format No. 2 trailer cards of the 100 and 400 series instructions optional. (See 3.1.5.)

- Increases number of symmetry cards from 48 to 96 . (See 3.2.3.)

- Increases number of atoms from 166 to 500. (See 3.2.4.)

- Makes entry of $\mathrm{VDC}_{2}$ on Type 6 and Type 7 temperature factor cards optional. (See 3.2.4.2.)

- Increases number of Format No. 2 trailer cards per instruction from 10 to 20. (See 3.3.1.)

- Adds a 205 instruction to change the plotting pen width. (See 3.3.3.)

- Makes "no retrace" (instruction 303) the default. (See 3.3.4.3.)

- Adds a 304 instruction to control the resolution (smoothing) of the ellipsoids. (See 3.3.4.4.)

- Allows atom screening on the 403/413 and 404/414 instructions. (See 3.3.5.3.)

- Changes 600 instructions to allow input of SCAL2 or ellipsoid probability. (See 3.3.7.)

- Adds a 706/716 instruction to add another standard ellipsoid type (open octant football) for drawing atoms. (See 3.3.8.)

- Adds lower case letters for labeling. (See 3.3.10.)

- Centers titles automatically if they begin in column 1 of Format No. 3 trailer cards following instructions 902, 903, and 913. (See 3.3.10.)

- Makes instruction number 1001 an alias for the 511 instruction of OR TEP-II. ${ }^{2}$ (See 3.3.12.)

\subsection{REPORT ORGANIZATION}

This report covers the following topics. First, Section 2.1 includes a summary table of the ORTEP instructions and is the part of the report to which the experienced user will routinely refer. The remainder of Section 2 provides an overview of how to program an ORTEP illustration, ending with a detailed example. Section 3 defines the terms used in this report and describes the ORTEP input in detail. Section 4 discusses the use of ORTEP-III in general terms while Section 5 looks at some of the more technical aspects. The mathematics of thermal-motion probability ellipsoids are described in Section 6. Lastly, several example structures illustrating a number of ORTEP's capabilities are provided in Section 7. The appendices provide brief descriptions of the subprograms and the more important variables that are used in ORTEP along with a listing of the entire program's FORTRAN code. 


\section{PROGRAMMING ORTEP}

\subsection{GENERAL PRINCIPLES}

Originally, the input to ORTEP was a set of punched cards. Now, the ORTEP input is a computer text file containing lines of information corresponding to the cards. However, since the input must generally be very precisely formatted as required by the FORTRAN code, it is still convenient to refer to the individual lines of input as "cards," and that practice is maintained in this report.

For those who may be unfamiliar with FORTRAN input, two points are in order. First, when a card field is specified to contain a particular numeric input item, the value should be entered in the rightmost positions of the field. Secondly, the value of " 0 " is assigned to a numeric input item whose card field is left blank.

ORTEP input consists of five types of information.

- Title card (see 3.2.1)

- Cell parameter card (see 3.2.2)

- Symmetry cards (see 3.2.3)

- Atomic parameter cards (see 3.2.4)

- Instruction cards (see Table 2.1 and 3.3)

ORTEP applies the concept of programming to the task of drawing illustrations. Using this approach, a set of basic building block operations have been developed that are put together by the user to "program" an illustration. These operations, which are termed instructions, control six types of activities.

- setting up the graphics

- composing an illustration

- drawing the illustration

- repeating a sequence of other instructions

- calculating and printing tables of ancillary information

- terminating the process

Table 2.1 is a summary of the ORTEP instructions. Each instruction starts on a separate card and consists of an identifying number and the parameters needed for the particular instruction. The general role of these instructions is explained in the remaining parts of this section, and the individual instructions are described in detail in Section 3.3. The simplest way to construct the program is, first, to select a "template" instruction set used for some previous related ORTEP drawing and then to scan through the list of instructions in Table 2.1 and pick out the relevant parameters and new instructions to modify the template.

In order to produce high quality illustrations with ORTEP, an iterative approach is generally required; that is, the illustration must usually be computed and plotted several times before an optimal figure is produced. With each trial, as many factors as possible are optimized to give a more informative and more aesthetically pleasing result. 
Table 2.1a. Summary table of Format No. 0 ORTEP-III instructions and Format No. 1 instruction continuation cards.

\begin{tabular}{|c|c|c|c|c|c|c|c|c|c|}
\hline Function & $1-3$ & $4-9$ & $10-18$ & $19-27$ & $28-36$ & $37-45$ & $46-54$ & $55-63$ & $64-72$ \\
\hline $\begin{array}{l}\text { Structure Analysis } \\
\text { Distances } \\
\text { (Format No. } 1 \text { trailer card) } \\
\text { Distances + angles } \\
\text { Principal axes } \\
\text { Distances single convolute } \\
\text { (Format No. } 1 \text { trailer card) } \\
\text { Dist. reiterate convolute }\end{array}$ & $\begin{array}{c}0,1 \text {, or } 2 \\
2 \\
0,1, \text { or } 2 \\
- \\
0,1, \text { or } 2 \\
2 \\
0,1, \text { or } 2\end{array}$ & $\begin{array}{l}101 \\
\frac{1}{102} \\
103 \\
105 \\
\frac{106}{106}\end{array}$ & $\begin{array}{l}\text { Org. ADR (f) } \\
- \\
\text { (same as 101) } \\
\text { Org. NR (f) } \\
- \\
\text { (same as } 105 \text { ) }\end{array}$ & $\begin{array}{l}\text { Org. ADR (t) } \\
\text { [LOGC] } \\
-\overline{N R}(t) \\
\text { Org. } \\
{[L O G C]}\end{array}$ & $\begin{array}{c}\text { Tar. ANR (f) } \\
- \\
\text { Tar. } \overline{N R}(f) \\
-\end{array}$ & $\begin{array}{c}\text { Tar. ANR (t) } \\
- \\
\text { Tar. } \overline{N R}(t) \\
-\end{array}$ & $\begin{array}{c}\mathrm{D}_{\max }(\AA) \\
- \\
- \\
\mathrm{D}_{\max }(\AA) \\
-\end{array}$ & $\begin{array}{c}- \\
- \\
\text { NR Type } \\
-\end{array}$ & E \\
\hline $\begin{array}{l}\text { Plotter Control } \\
\text { Initialize } \\
\text { Shift plot origin/terminate } \\
\text { Color } \\
\text { Pen width } \\
\end{array}$ & $\begin{array}{l}- \\
- \\
-\end{array}$ & $\begin{array}{l}201 \\
202 \\
204 \\
205 \\
\end{array}$ & $\begin{array}{l}{[\bar{X} \text { (in.) }]} \\
\text { ICOLOR } \\
\text { WIDTH }\end{array}$ & {$\left[\begin{array}{c}\overline{(i n} .) \\
- \\
-\end{array}\right.$} & $\begin{array}{l}- \\
- \\
-\end{array}$ & $\begin{array}{l}- \\
- \\
-\end{array}$ & $\begin{array}{l}- \\
\overline{-}\end{array}$ & $\begin{array}{l}- \\
- \\
-\end{array}$ & $\begin{array}{l}- \\
- \\
-\end{array}$ \\
\hline $\begin{array}{l}\text { Drawing Parameters } \\
\text { Dimensions and view } \\
\text { Title rotation } \\
\text { Retrace displace } \\
\text { Ellipse smoothness }\end{array}$ & $\begin{array}{l}- \\
- \\
-\end{array}$ & $\begin{array}{l}301 \\
302 \\
303 \\
304\end{array}$ & $\begin{array}{l}\text { X (in.) } \\
\text { THETA ( }) \\
\text { DISP (in.) } \\
\text { CHORD }\end{array}$ & $\begin{array}{l}Y \text { (in.) } \\
- \\
-\end{array}$ & $\begin{array}{c}\text { VIEW (in.) } \\
- \\
-\end{array}$ & $\begin{array}{c}\text { BRDR (in.) } \\
- \\
-\end{array}$ & $\begin{array}{l}- \\
- \\
-\end{array}$ & $\begin{array}{l}- \\
- \\
-\end{array}$ & $\begin{array}{l}- \\
- \\
-\end{array}$ \\
\hline $\begin{array}{l}\text { ATOMS Array } \\
\text { Run add }\end{array}$ & 0 or 1 & 401 & FROM (1) & $(-) \mathrm{TO}(1)$ & [FROM (2) & $(-)$ TO (2)] & [FROM (3) & $(-) \mathrm{TO}(3)]$ & $\ldots$ \\
\hline $\begin{array}{l}\text { Sphere add } \\
\text { (Format No. } 1 \text { trailer card) } \\
\text { Sphere subtract }\end{array}$ & $\begin{array}{l}0,1, \text { or } 2 \\
2 \\
0,1, \text { or } 2\end{array}$ & $\frac{402}{412}$ & $\begin{array}{c}\text { Org. ADR (f) } \\
- \\
\text { (same as 402) }\end{array}$ & $\begin{array}{l}\text { Org. ADR (t) } \\
\text { [LOGC] }\end{array}$ & $\begin{array}{c}\text { Tar. ANR (f) } \\
-\end{array}$ & $\begin{array}{c}\text { Tar. ANR }(t) \\
-\end{array}$ & $\mathrm{D}_{\max }(\AA)$ & $\overline{-}$ & $\overline{-}$ \\
\hline $\begin{array}{l}\text { Box add } \\
\text { (Format No. } 1 \text { trailer card) } \\
\text { Box subtract }\end{array}$ & $\begin{array}{l}0,1 \text {, or } 2 \\
2 \\
0,1, \text { or } 2\end{array}$ & $\frac{403}{413}$ & $\begin{array}{l}\text { Org. ADR (f) } \\
- \\
\text { (same as 403) }\end{array}$ & $\begin{array}{l}\text { Org. ADR }(t) \\
\text { [LOGC] }\end{array}$ & Tar. ANR (f) & Tar. ANR (t) & $\stackrel{a}{a} 2(\AA)$ & $\mathrm{b} / 2(\AA)$ & $\mathrm{c} / 2(\AA)$ \\
\hline $\begin{array}{l}\text { Triclinic box add } \\
\text { (Format No. } 1 \text { trailer card) }\end{array}$ & $\begin{array}{c}0,1, \text { or } 2 \\
2\end{array}$ & $\begin{array}{l}404 \\
-\end{array}$ & $\begin{array}{c}\text { Org. } A D R(f) \\
-\end{array}$ & $\begin{array}{l}\text { Org. } A D R(t) \\
\text { [LOGC] }\end{array}$ & Tar. ANR ( $f)$ & Tar. ANR (t) & $a / 2$ (fract.) & $\mathrm{b} / 2$ (fract.) & $c / 2$ (fract.) \\
\hline $\begin{array}{l}\text { Triclinic box subtract } \\
\text { Convolute add } \\
\text { (Format No. } 1 \text { trailer card) }\end{array}$ & $\begin{array}{c}0,1, \text { or } 2 \\
0,1 \text {, or } 2 \\
\quad 2\end{array}$ & $\begin{array}{c}414 \\
405 \\
-\end{array}$ & $\begin{array}{l}\text { (same as 404) } \\
\text { Org. NR (f) } \\
\quad-\end{array}$ & $\begin{array}{l}\text { Org. NR (t) } \\
\text { [LOGC] }\end{array}$ & $\begin{array}{c}\text { Tar. NR (f) } \\
-\end{array}$ & Tar. NR (t) & $\mathrm{D}_{\max }(\AA)$ & $\begin{array}{l}\text { NR Type } \\
-\end{array}$ & - \\
\hline $\begin{array}{l}\text { Convolute subtract } \\
\text { Reiterate convolute add }\end{array}$ & $\begin{array}{l}0,1, \text { or } 2 \\
0,1 \text {, or } 2 \\
2\end{array}$ & $\begin{array}{r}415 \\
406 \\
-\end{array}$ & $\begin{array}{l}\text { (same as 405) } \\
\text { Org. NR (f) }\end{array}$ & $\begin{array}{l}\text { Org. NR }(t) \\
\text { IOGCl }\end{array}$ & Tar. NR (f) & Tar. NR (t) & $D_{\max }(\AA)$ & NR Type & - \\
\hline $\begin{array}{l}\text { Reiterate convolute subt. } \\
\text { Zero ATOMS array }\end{array}$ & 0,1, or 2 & $\begin{array}{l}416 \\
410\end{array}$ & $\begin{array}{c}\text { (same as 406) } \\
\text { - }\end{array}$ & & - & - & & & - \\
\hline
\end{tabular}


Table 2.1a. Summary table of Format No. 0 ORTEP-III instructions and Format No. 1 instruction continuation cards.

\begin{tabular}{|c|c|c|c|c|c|c|c|c|c|}
\hline Function & $1-3$ & 4-9 & $10-18$ & $19-27$ & $28-36$ & $37-45$ & $46-54$ & $55-63$ & $64-72$ \\
\hline $\begin{array}{l}\text { Cartesian System } \\
\text { Explicit definition } \\
\text { Rotate reference } \\
\text { Rotate working } \\
\text { Translate reference } \\
\text { Origin at centroid } \\
\text { Centroid org./inertial axes }\end{array}$ & $\begin{array}{l}\overline{0} \\
\overline{0} 1 \\
\overline{0} \text { or } 2 \\
0 \text { or } 2\end{array}$ & $\begin{array}{l}501 \\
502 \\
503 \\
504 \\
505 \\
506 \\
\end{array}$ & $\begin{array}{l}\text { ORGN } \\
\text { Axis No. } \\
\text { Axis No. } \\
\Delta \mathrm{X} \text { (in.) } \\
-\end{array}$ & $\begin{array}{l}V 1(f) \\
\left.\text { Rotation ( }{ }^{\circ}\right) \\
\left.\text { Rotation ( }{ }^{\circ}\right) \\
\Delta Y \text { (in.) } \\
- \\
-\end{array}$ & $\begin{array}{l}\mathrm{VI}(\mathrm{t}) \\
\text { [Axis No. } \\
- \\
\Delta \mathrm{Z} \text { (in.) } \\
-\end{array}$ & $\begin{array}{c}\text { V2(f) } \\
\text { Rotation] } \\
- \\
- \\
- \\
-\end{array}$ & $\begin{array}{l}\mathrm{V} 2(\mathrm{t}) \\
\cdots \\
= \\
= \\
\end{array}$ & $\begin{array}{l}- \\
\cdots \\
- \\
- \\
-\end{array}$ & $\begin{array}{l}\text { Type } \\
\cdots \\
= \\
= \\
=\end{array}$ \\
\hline $\begin{array}{l}\text { Position and Scale } \\
\text { Explicit center and scale } \\
\text { Explicit center and auto scale } \\
\text { Explicit scale and auto center } \\
\text { Auto center and scale } \\
\text { Incr. position and incr. scale } \\
\text { Incr. position and auto scale } \\
\text { Incr. scale and auto center }\end{array}$ & $\begin{array}{l}\overline{-} \\
\overline{-} \\
\bar{z} \\
\bar{z}\end{array}$ & $\begin{array}{l}601 \\
602 \\
603 \\
604 \\
611 \\
612 \\
613 \\
\end{array}$ & $\begin{array}{l}\mathrm{X} 0 \text { (in.) } \\
\mathrm{X} 0 \text { (in.) } \\
- \\
\Delta \mathrm{X} 0 \text { (in.) } \\
\Delta \mathrm{X} 0 \text { (in.) } \\
-\end{array}$ & $\begin{array}{l}\text { Y0 (in.) } \\
\text { Y0 (in.) } \\
- \\
- \\
\triangle \mathrm{YO} \text { (in.) } \\
\triangle \mathrm{YO} \text { (in.) } \\
-\end{array}$ & $\begin{array}{c}\text { SCAL1 } \\
\overline{-} \\
\text { SCAL1 } \\
- \\
\triangle S C A L 1 \\
\overline{-} \\
\triangle S C A L 1\end{array}$ & $\begin{array}{l}\text { SCAL2* } \\
\text { SCAL2* } \\
\text { SCAL2* } \\
\text { SCAL2* } \\
\text { SCAL2* } \\
\text { SCAL2* } \\
\text { SCAL2* }\end{array}$ & $\begin{array}{l}z \\
z \\
z \\
=\end{array}$ & $\begin{array}{l}- \\
z \\
z \\
-\end{array}$ & $\begin{array}{l}\bar{z} \\
\bar{z} \\
\bar{z}\end{array}$ \\
\hline $\begin{array}{l}\text { Atom Plotting } \\
\text { Shaded octant football } \\
\text { (Format No. } 1 \text { trailer card) } \\
\text { Football } \\
\text { Open model } \\
\text { Boundary only } \\
\text { Explicit ellipsoid description } \\
\text { (Format No. } 1 \text { trailer card) } \\
\text { Open octant football } \\
\text { As above except } \\
\text { no printed output of } \\
\text { individual coordinates }\end{array}$ & $\begin{array}{l}0 \text { or } 1 \\
0 \text { or } 1 \\
0 \text { or } 1 \\
0 \text { or } 1 \\
0 \text { or } 1 \\
- \\
0 \text { or } 1 \\
0 \text { or } 1 \\
0 \text { or } 1 \\
0 \text { or } 1 \\
0 \text { or } 1 \\
0 \text { or } 1 \\
0 \text { or } 1\end{array}$ & $\begin{array}{c}701 \\
-702 \\
703 \\
704 \\
705 \\
- \\
706 \\
711 \\
712 \\
713 \\
714 \\
715 \\
716\end{array}$ & $\begin{array}{c}\overline{-} \\
\text { A0 (in.) } \\
\text { (same as 701) } \\
\text { (same as 701) } \\
\text { (same as 701) } \\
\text { NPLANE } \\
\text { A0 (in.) } \\
\text { (same as 701) } \\
\text { (same as 701) } \\
\text { (same as 701) } \\
\text { (same as 701) } \\
\text { (same as 701) } \\
\text { (same as 705) } \\
\text { (same as 701) }\end{array}$ & A1 (in.) & $\begin{array}{l}\text { NLINE } \\
\text { NR (f) }\end{array}$ & $\begin{array}{l}\text { NDASH } \\
\text { NR (t) }\end{array}$ & $\begin{array}{l}\text { Sym. hgt. (in.) } \\
\text { NR Type }\end{array}$ & II Offset (in.) & $\perp$ Offset (in.) \\
\hline
\end{tabular}

*Or probability (entered as negative whole number). 
Table 2.1a. Summary table of Format No. 0 ORTEP.III instructions and Format No. 1 instruction continuation cards.

\begin{tabular}{|c|c|c|c|c|c|c|c|c|c|}
\hline Function & $1-3$ & $4-9$ & $10-18$ & $19-27$ & $28-36$ & $37-45$ & $46-54$ & $55-63$ & $64-72$ \\
\hline $\begin{array}{l}\text { Bond Plotting } \\
\text { Explicit } \\
\text { Implicit stick } \\
\text { Implicit line } \\
\text { As above except } \\
\text { no printed output }\end{array}$ & $\begin{array}{c}{\left[\begin{array}{ll}1 & \&\end{array}\right] 2} \\
2 \\
2 \\
{\left[\begin{array}{ll}1 & \&\end{array}\right] 2} \\
2 \\
2\end{array}$ & $\begin{array}{l}801 \\
802 \\
803 \\
811 \\
812 \\
813\end{array}$ & $\begin{array}{c}\quad \mathrm{ADC}(\mathrm{f}) \\
\quad- \\
\text { - } \\
\text { (same as } 801 \text { ) } \\
\text { (same as 802) } \\
\text { (same as 803) }\end{array}$ & $\begin{array}{l}\text { ADC }(t) \\
\text { NR Type } \\
\text { NR Type }\end{array}$ & $\begin{array}{c}{[\mathrm{ADC}(\mathrm{f})} \\
- \\
-\end{array}$ & $\begin{array}{c}\operatorname{ADC}(t)] \\
-\end{array}$ & $\begin{array}{c}{[\mathrm{ADC}(\mathrm{f})} \\
- \\
-\end{array}$ & $\begin{array}{c}\operatorname{ADC}(\mathrm{t})] \\
- \\
-\end{array}$ & - \\
\hline $\begin{array}{l}\text { Label Plotting } \\
\text { Atom label } \\
\text { Regular title } \\
\text { Normal plane. vector title } \\
\text { General plane vector title } \\
\text { Nrm. plane bond-length label } \\
\text { (1 decimal place) } \\
\text { ( } 2 \text { decimal places) } \\
\text { ( } 3 \text { decimal places) } \\
\text { Gen. plane bond-length label } \\
\text { (1 decimal place) } \\
\text { ( } 2 \text { decimal places) } \\
\text { ( } 3 \text { decimal places) }\end{array}$ & $\begin{array}{l}-3 \\
3 \\
3 \\
- \\
- \\
- \\
- \\
-\end{array}$ & $\begin{array}{l}901 \\
902 \\
903 \\
913 \\
\\
904 \\
905 \\
906 \\
\\
\\
914 \\
915 \\
916\end{array}$ & $\begin{array}{c}\mathrm{ADC} 1 \\
\mathrm{ADC} 1 \\
\mathrm{ADC} 1 \\
\mathrm{ADC} 1 \\
\\
\text { ADC 1 } \\
\text { (same as 904) } \\
\text { (same as 904) } \\
\\
\text { ADC 1 } \\
\text { (same as 914) } \\
\text { (same as 914) }\end{array}$ & $\begin{array}{l}{[\mathrm{ADC} 2]} \\
{[\mathrm{ADC} 2]} \\
\mathrm{ADC} 2 \\
\mathrm{ADC} 2 \\
\mathrm{ADC} 2\end{array}$ & $\begin{array}{l}\text { X Reset (in.) } \\
\text { X Reset (in.) } \\
\text { X Reset (in.) } \\
\quad- \\
\text { X Reset (in.) }\end{array}$ & $\begin{array}{l}\text { Y Reset (in.) } \\
\text { Y Reset (in.) } \\
\text { Y Reset (in.) } \\
\quad- \\
\text { Y Reset (in.) }\end{array}$ & $\begin{array}{l}\text { HGT (in.) } \\
\text { HGT (in.) } \\
\text { HGT (in.) } \\
\text { HGT (in.) } \\
\text { HGT (in.) } \\
\text { HGT (in.) }\end{array}$ & $\begin{array}{l}\text { "I Offset (in.) } \\
\text { " Offset (in.) } \\
\text { ॥ Offset (in.) } \\
\text { "I Offset (in.) } \\
\text { " Offset (in.) } \\
\text { " Offset (in.) }\end{array}$ & $\begin{array}{l}\perp \text { Offset (in.) } \\
\perp \text { Offset (in.) } \\
\perp \text { Offset (in.) } \\
\perp \text { Offset (in.) } \\
\perp \text { Offset (in.) } \\
\perp \text { Offset (in.) }\end{array}$ \\
\hline $\begin{array}{l}\text { Overlap Correction } \\
\text { Atoms [and implicit bonds] } \\
\text { (Format No. } 1 \text { trailer card) } \\
\text { Explicit bonds } \\
\text { Implicit bonds }\end{array}$ & $\begin{array}{c}0 \text { [or 2] } \\
{\left[\begin{array}{cc}1 & \&] 2 \\
2\end{array}\right.} \\
\end{array}$ & $\begin{array}{l}1001 \\
821 \\
822 \\
\end{array}$ & $\begin{array}{c}0,1, \text { or } \\
\text { OVMRGN (in.) } \\
\text { ADC (f) } \\
-\end{array}$ & $\begin{array}{l}\text { NR Type } \\
\text { ADC (t) } \\
\text { NR Type }\end{array}$ & $\begin{array}{c}- \\
{[\mathrm{ADC}(\mathrm{f})} \\
-\end{array}$ & $\begin{array}{c}- \\
\mathrm{ADC}(\mathrm{t})] \\
-\end{array}$ & $\begin{array}{c}- \\
{[\mathrm{ADC}(\mathrm{f})} \\
- \\
\end{array}$ & $\begin{array}{c}- \\
\operatorname{ADC}(t)] \\
-\end{array}$ & - \\
\hline $\begin{array}{l}\text { Save Sequence } \\
\text { Start } \\
\text { End } \\
\text { Execute }\end{array}$ & - & $\begin{array}{l}1101 \\
1102 \\
1103\end{array}$ & - & $\begin{array}{l}- \\
-\end{array}$ & - & $\begin{array}{l}- \\
- \\
-\end{array}$ & - & $\begin{array}{l}- \\
- \\
-\end{array}$ & $\begin{array}{l}- \\
- \\
-\end{array}$ \\
\hline $\begin{array}{l}\text { Terminate } \\
\text { Next Structure }\end{array}$ & - & $\begin{array}{l}-1 \\
-2\end{array}$ & - & 二 & 二 & 二 & 二 & 二 & 二 \\
\hline
\end{tabular}


Table 2.1b. Summary table of Format No. 2 instruction continuation cards.

\begin{tabular}{|c|c|c|c|c|c|c|c|c|}
\hline \multirow{2}{*}{ Columns } & \multirow[b]{2}{*}{$\begin{array}{c}101,102,105 \\
106,402 / 412 \\
405 / 415 \\
406 / 416\end{array}$} & \multirow[b]{2}{*}{$\begin{array}{c}403 / 413 \\
404 / 414\end{array}$} & \multirow[b]{2}{*}{505,506} & \multicolumn{3}{|c|}{ Positive Number or Blank in Col. $43-48$} & \multicolumn{2}{|c|}{ Negative Number in Col. $43-48$} \\
\hline & & & & $801 / 811,821$ & $802 / 812,822,1001$ & $803 / 813$ & $\begin{array}{l}802 / 812, \\
822,1001\end{array}$ & $803 / 813$ \\
\hline 3 & 0 or 2 & 0 or 2 & 0 or 2 & 0 or 2 & 0 or 2 & 0 or 2 & 0 or 2 & 0 or 2 \\
\hline $4-9$ & 一 & 一 & - & 一 & - & - & - & 一 \\
\hline $10-12$ & [Org. NR (f) & [Org. NR (f) & $N R(f)$ & 一 & Org. NR (f) & Org. NR (f) & Org. NR (f) & Org. NR (f) \\
\hline $13-15$ & Org. NR $(t)]$ & Org. NR (t)] & NR $(t)$ & - & Org. NR (t) & Org. NR (t) & Org. NR (t) & Org. NR (t) \\
\hline $16-18$ & [Tar. NR (f) & [Tar. NR (f) & 一 & - & Tar. NR (f) & Tar. NR (f) & Tar. NR (f) & Tar. NR (f) \\
\hline $19-21$ & Tar. NR (t)] & Tar. NR (t)] & - & - & Tar. NR (t) & Tar. NR (t) & Tar. NR (t) & Tar. NR (t) \\
\hline $22-24$ & [NR type] & NR type & NR type & Bond type & Bond type & - & Bond type & 一 \\
\hline $25-30$ & {$\left[\mathrm{D}_{\min }(\AA)\right.$} & 一 & Weight & - & $\mathrm{D}_{\min }(\AA)$ & $\mathrm{D}_{\min }(\AA)$ & $\mathrm{D}_{\min }(\AA)$ & $\mathrm{D}_{\min }(\AA)$ \\
\hline $31-36$ & $\left.\mathrm{D}_{\max }(\AA)\right]$ & - & - & - & $\mathrm{D}_{\max }(\AA)$ & $\mathrm{D}_{\max }(\AA)$ & $\mathrm{D}_{\max }(\AA)$ & $\mathrm{D}_{\max }(\AA)$ \\
\hline $37-42$ & 一 & - & 一 & Bond radius $(\AA)$ & Bond radius $(\AA)$ & 一 & Bond radius $(\AA)$ & - \\
\hline $43-48$ & 一 & - & - & Persp. label hgt. (in.) & Persp. label hgt. (in.) & - & Poly. NR (f) & Poly. NR (f) \\
\hline $49-54$ & - & - & - & $\perp$ displacement (in.) & $\perp$ displacement (in.) & - & Poly. NR (t) & Poly. NR (t) \\
\hline $55-60$ & 一 & 一 & - & Nonp. label hgt. (in.) & Nonp. label hgt. (in.) & - & Poly. $D_{\min }(\AA)$ & Poly. $D_{\min }(\AA)$ \\
\hline $61-66$ & - & - & - & $\perp$ displacement (in.) & $\perp$ displacement (in.) & - & Poly. $D_{\max }(\AA)$ & Poly. $D_{\max }(\AA)$ \\
\hline $67-72$ & - & - & - & Digits indicator & Digits indicator & - & 一 & - \\
\hline
\end{tabular}




\subsection{PROGRAMMING A NONSTEREOSCOPIC ILLUSTRATION FOR ORTEP}

This section describes the general stepwise procedure to follow when writing an ORTEP program to draw a single nonstereographic illustration of the contents of one unit cell. The instruction numbers used are examples only, and often other instructions may be used instead.

\subsubsection{Graphics Setup}

The first instruction card is instruction 201 (see 3.3.3), which initializes plotting.

Next, instruction 301 (see 3.3.4.1) is needed to set the following drawing parameters: $x$ dimension for the plot boundary, $y$ dimension for the plot boundary, viewing distance for perspective projection (or " 0 " as a signal for parallel projection), and border (or margin) dimension inside the boundary. All values are supplied in inches.

\subsubsection{Composing the Illustration}

This step involves specifying which atoms are to be used as the figure subject, the rotational orientation of the figure, and the scaling and positioning of the figure relative to the drawing area. These three components of composition are implemented by the 400,500 , and 600 series instructions, respectively.

Atoms can be explicitly added to the figure with a 401 instruction (see 3.3.5.1). For unit cell content drawings, the 404 instruction (see 3.3.5.3) is useful. It defines a triclinic box of enclosure, and ORTEP determines which atoms appear in the figure.

A 501 instruction (see 3.3.6.1) can be used to orient the crystal axes relative to the $x$ and $y$ axes of the plot. If additional adjustment of the figure orientation is necessary, a 502 instruction (see 3.3.6.2) can be used after the 501 .

Scaling and positioning of the figure to fill the drawing area can be accomplished automatically with a 604 instruction (see 3.3.7.1).

\subsubsection{Drawing the Illustration}

Crystal structure illustrations of the ball-and-stick type are made up of three components: balls (atoms), sticks (bonds), and labels. The three components are drawn with the 700, 800, and 900 instruction series, respectively; the first two instruction series can also perform certain types of labeling. Before drawing any atoms or bonds, instruction 1001 (or 511) (see 3.3.12) should be used to calculate and store the information needed for the overlap correction.

The atom representation can be either a general ellipsoid or a boundary ellipse. In some cases, these become a sphere and a circle. Chemical symbols may be plotted simultaneously with the atoms. A 704 instruction (see 3.3.8) will draw circles for all the atoms of the subject and put the chemical symbols within the circles.

Bonds are not always necessary in a drawing; but for structures with molecules or with distinctive groupings, they are usually quite helpful. The most convenient method for describing and drawing bonds is instruction 812 (see 3.3.9.2). This instruction uses vector search codes (see 3.1.5) that reflect the user's knowledge of the structural chemistry and the interatomic distance ranges for the compound being drawn. Covalent bonds or any other desired type are found and 
drawn automatically from the list of atoms that make up the subject. If desired, the interatomic distance label can also be drawn with the bond.

Various types of labeling can be done with the 900 series instruction. The one that will most often be included is a caption for the figure, provided by the 902 instruction (see 3.3.10).

\subsubsection{Terminating the Drawing of the Illustration}

Instruction 202 with no parameters (see 3.3.3) terminates the plotting.

To terminate ORTEP, a -1 instruction (see 3.3.13) is used as the last instruction of the input.

\subsection{PROGRAMMING A STEREOSCOPIC ILLUSTRATION FOR ORTEP}

A stereoscopic pair of figures is simply two perspective views of the subject as seen from two different viewpoints (which are usually $5^{\circ}-6^{\circ}$ apart). This pair is produced with ORTEP by programming for two drawings. A few instructions in addition to those outlined in Section 2.2 are needed for producing stereo figures. These are the stereoscopic rotation instruction 503 and the 1100 series of instructions that are used to repeat a series of instructions. A program to draw a stereo pair would involve the following steps.

1. set up the graphics

2. compose the subject

3. stereo rotate subject for left-eye view

4. store overlap information

5. draw the subject

6. shift plot origin for second view

7. stereo rotate subject for right-eye stereo view

8. store overlap information

9. draw the subject

10. terminate plotting

11. terminate ORTEP

\subsubsection{Stereoscopic Rotations}

In general, one member of a detailed stereoscopic illustration cannot be drawn independently of the other member of the pair because certain features (e.g., which octant of an ellipsoid is shaded) must be done identically in the two drawings. In ORTEP the "stereoscopically sensitive decisions" are handled by using two Cartesian coordinate systems: the reference system and the working system (see 3.1.8). The steps involved in picture composition (see 2.2.2) and the stereoscopically sensitive decisions are always based on the reference system, but the drawing of the illustration (see 2.2.3) is always based on the working system. A stereoscopic rotation is simply a rotation of the working system from the reference system about the axis that is vertical while viewing the final result. For example, a nominal rotation of $+2.7^{\circ}$ about the plot's $y$ axis might be used for the left-eye view and a rotation of $-2.7^{\circ}$ about the same axis might be made before plotting the right-eye view, thus producing a total interocular angle of $5.4^{\circ}$. 


\subsubsection{Repeating a Sequence of Operations}

It should be noted that steps 8 and 9 in the program to draw a stereo pair, which actually comprise several ORTEP instructions, are identical to steps 4 and 5. The program can be shortened somewhat by using the "save sequence" instructions (see 3.3.11). An 1101 instruction (start save sequence) would be placed before step 4, and an 1102 instruction (end save sequence) after 5. Then steps 8 and 9 can be replaced by a single 1103 instruction (execute save sequence).

Any sequence of instructions can be saved in this manner and repeated as many times as desired with 1103 instructions. For example, the save sequence feature can be used to produce a complete series of views of a structure at $15^{\circ}$ intervals about an axis. Note that the instructions between the start and end instructions are both executed and saved the first time through.

\subsection{DRAWING THE CUBANE STRUCTURE: AN EXAMPLE}

The standard example for illustrating the use of ORTEP is the novel compound cubane $\left(\mathrm{C}_{8} \mathrm{H}_{8}\right)$, whose structure was published by Fleischer in $1964 .{ }^{5}$ In cubane, the carbon-carbon bonds lie along the edges of a cube within experimental error. The compound crystallizes with the trigonal symmetry of space group $\mathrm{R} \overline{3}$. The $\overline{3}$ axis lies along a body diagonal of the molecule, and as a result the compound contains only four unique atoms. These are one carbon and its attached hydrogen in general positions off the $\overline{3}$ axis ( $\mathrm{C} 1$ and $\mathrm{H} 1)$ and one carbon and its hydrogen in special positions on the 3 axis ( $\mathrm{C} 2$ and $\mathrm{H} 2$ ). Anisotropic temperature factor coefficients were fitted to the carbon atoms during the least-squares refinement of the structure, and isotropic temperature factors were used for the hydrogen atoms. The anisotropic temperature factors given for the carbon atoms are of the type called zero ${ }^{\dagger}$ in this report (see 3.2.4.2).

To draw the structure the following information is needed:

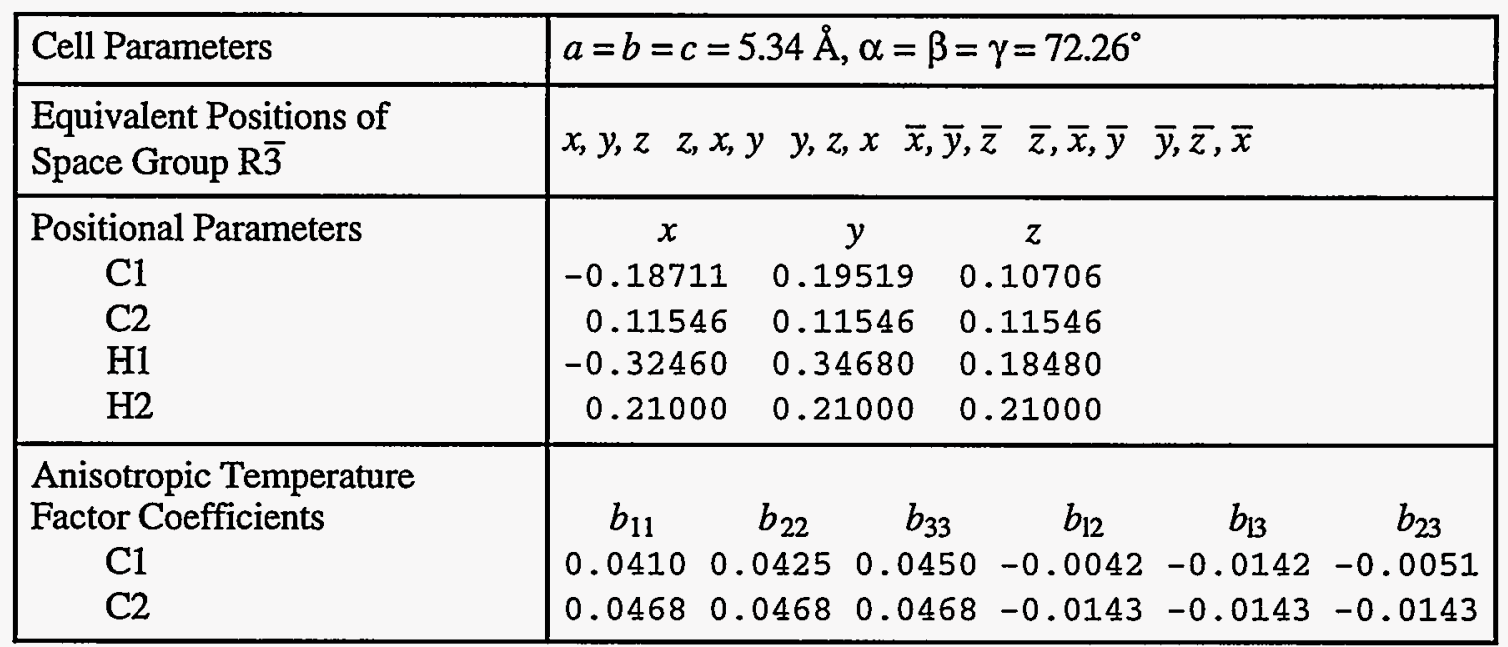

tOccasionally authors of structure papers neglect to define the equation for the anisotropic temperature factor coefficients. In the present case, the type can be determined from the comparative isotropic temperature factor listed in anisotropic form in Fleischer's Table II. ${ }^{5}$ In other instances, one must arbitrarily choose a type (usually 0,1 , or 4 in the USA), do the principal axis transformation, and then check that the principal values are correct, or at least reasonable. In particular, the principal values must all be positive. 


\subsubsection{Data Input for Cubane}

\subsubsection{Title Card}

The first line of input is a job title (see 3.2.1).

CUBANE, E.B. FLEISCHER, J. AM. CHEM. SOC., 86, 3889 (1964)

\subsubsection{Cell Parameter Card}

Cell parameters are provided on the second line (see 3.2.2). The "1" in the first column signals the format of the symmetry information that follows.
$1 \quad 5.34$
5.34
5.34
72.26
72.26
72.26

\subsubsection{Symmetry Cards}

The symmetry operators of the space group begin on line 3 of the input (see 3.2.3). The last of these has a numeric value other than " 0 " in column 1.

$$
\begin{gathered}
x, y, z \\
z, x, y \\
y, z, x \\
-x,-y,-z \\
-z,-x,-y \\
1-y,-z,-x
\end{gathered}
$$

\subsubsection{Atomic Parameter Cards}

Two cards are required for each atom. The first gives the atom's positional parameters (see 3.2.4.1), and the second provides its thermal parameters (see 3.2.4.2). The last atom has a " 1 " in column 1 of its thermal parameter card. Atoms are frequently referenced in the ORTEP instructions by their numeric position within this list.

Atoms 1 and 2 are entered with positional parameter Type 0 and anisotropic temperature factor Type 0 .

$\begin{array}{lllrrrr}\mathrm{C} 1 & & & -.18711 & .19519 & .10706 & 0 \\ 0 \quad .04100 & .04250 & .04500 & -.00420 & -.01420 & -.00510 & 0 \\ \mathrm{C} 2 & & & .11546 & .11546 & .11546 & 0 \\ 0 \quad .04680 & .04680 & .04680 & -.01430 & -.01430 & -.01430 & 0\end{array}$

Atoms 3 and 4 are entered with positional parameter Type 0 and with dummy sphere temperature factors (Type 7) with a radius of $0.1 \AA$ before scaling.

$\begin{array}{llllll}0^{\mathrm{H} 1} & & -.32460 & .34680 & .18480 & 0 \\ { }^{\mathrm{H} 2} & .10 & .21000 & .21000 & .21000 & 7 \\ & .10 & & & & 7\end{array}$


A dummy atom (atom 5) at the cell origin is also included with a blank card dummy sphere. This could also be entered with Type 7 as were atoms 3 and 4 .

$\begin{array}{lllll}\text { ORGN } & 0 & 0 & 0 & 0 \\ 1 & & & & 0\end{array}$

\subsubsection{Analysis of Structure}

The 100 series instructions (see 3.3.2) are neither associated with nor required for producing an illustration. They are shown here to demonstrate how they are used. A 101 instruction is used to obtain a tabulation of the atoms surrounding one atom or a series of several designated atoms. For example, to obtain a list of all atoms (hydrogen and carbon atoms) out to a distance of $3.61 \AA$ about the two carbons $\mathrm{C} 1$ and $\mathrm{C} 2$, the following 101 instruction would be used.

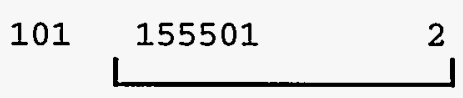

(a)

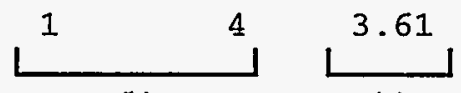

(b)

(c)

where the parts designate:

(a) origin atoms 1 through 2 of symmetry operation 1 ,

(b) target atoms 1 through 4 of all symmetry and translation operations, and

(c) a distance $D_{\max }$ of $3.61 \AA$.

A 102 instruction gives both interatomic distances and interatomic angles. The following instruction could be used to find all covalent bonds and bond angles about the two carbons.

$\begin{array}{llllll}102 & 155501 & 2 & 1 & 4 & 1.8\end{array}$

In this case a smaller $D_{\max }$ is used so that only the distances and angles of immediate interest would be computed since there are $n(n-1) / 2$ angles for $n$ interatomic vectors about an atom.

\subsubsection{Programming the Cubane Ilustration}

First, plotting is initialized with a 201 instruction (see 3.3.3).

201

The two plot boundary dimensions can be equal for the present illustration since the cubane molecule is a cube. A $2.8 \times 2.8$ inch boundary is specified with a 0.25 inch margin to give a $2.3 \times 2.3$ inch square working area. A 10 inch view distance might be reasonable to use in viewing a model of this size. These are set with a 301 instruction. (See 3.3.4.1.)

301

$$
2.8 \quad 2.8 \quad 10 \text {. }
$$$$
.25
$$

The subject of the illustration is a single complete cubane molecule. Since all the atoms of the molecule were not provided in the input atoms list, the "missing" atoms may be found by using a 402 instruction to specify a sphere of enclosure, centered on the dummy atom 5, which is at the center of a cubane molecule. A radius of $3.2 \AA$ should be adequate to find all the atoms and 
isolate a single molecule. The atoms found by this instruction are stored in the ATOMS array. (See 3.3.5.2.)

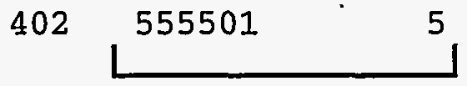

(a)

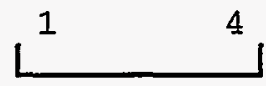

(b)

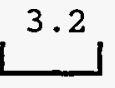

(c)

where the parts designate:

(a) a run of origin atom(s) representing sphere centers from atom 5 to atom 5 in symmetry position 55501 (in this example, a single sphere),

(b) a run of target atoms from atom 1 to atom 4, and

(c) a sphere radius of $3.2 \AA$.

A 501 instruction is used to establish a coordinate system for orienting the molecule. In this case, the coordinate system is defined along the edges of the cubane cube. The origin is positioned on the dummy atom 5 . The desired coordinate system orientation is defined by specifying two vectors from the special position atom 255501 to the two symmetry-related general-position atoms 155501 and 155502. (See 3.3.6.1.)

$\begin{array}{llllllll}501 & 555501 & 255501 & 155501 & 255501 & 155502 & 0 & 0\end{array}$

A 502 instruction is used to rotate the molecule relative to the established coordinate system to optimize the appearance of the illustration. A rotation of $25^{\circ}$ about the $y$ axis (axis 2) followed by a rotation of $28^{\circ}$ about the $x$ axis (axis 1 ) will produce a satisfactory view of the molecule. (See 3.3.6.2.)

$\begin{array}{lllll}502 & 2 & 25 . & 1 & 28 .\end{array}$

To position and scale the subject for projection onto the "drawing board" and to utilize all available space, a 604 instruction is used, which automatically sets the origin and drawing scale. It also sets the ellipsoid scale factor ratio SCAL2 to the value corresponding to $50 \%$ probability unless a non-zeto value is supplied on the instruction card. (See 3.3.7.1).
604
0
0
0
0

The stereoscopic rotation for the left eye is specified next with a 503 instruction. A rotation of $2.7^{\circ}$ about the $y$ axis (axis 2 ) is used. Later in the program, $a-2.7^{\circ}$ rotation about the same axis will be made for the right-eye view to give a total interocular angle of 5.4 ${ }^{\circ}$. (See 3.3.6.3.)

$\begin{array}{lll}503 & 2 & 2.7\end{array}$

Since the structure will be drawn twice, once for each eye, the "save sequence" feature is used to shorten the program. Note that the instructions between the start (1101) and end (1102) are both executed and saved the first time through. They can then be re-executed as many times as desired by using the "execute save sequence" instruction 1103. (See 3.3.11.) The 1101 instruction starts the save sequence. 
The 1001 (or 511) instruction stores the information needed for the overlap hidden-line correction (i.e., as a projected boundary ellipse for each atom in the ATOMS array and a quadrangle approximation for each bond). The bonds are specified with a Format No. 2 trailer card identical to that used in the 812 instruction described below. (See 3.3.12.1.)

21001

$$
\begin{array}{llllllll}
1 & 4 & 1 & 4 & 4 & 0.9 & 1.6 & .04
\end{array}
$$

The ATOMS array currently contains all the atom designators for one cubane molecule. The molecule is drawn in two separate steps so that the hydrogen and carbon atoms can be given different graphical representations. To draw the carbon atoms (ANR =1,2), a standard model produced with the 716 instruction (new in ORTEP-III) is used. [This model is the same as that produced with a 705/715 instruction that (a) draws the three principal-plane forward traces and the boundary-plane trace (NPLANE $=4$ ), (b) omits the reverse sides of the principal planes (NDOT = 0 ), (c) draws the forward principal axes without additional shading (NLINE =1), and (d) omits the reverse principal axes (NDASH $=0$ ).] In addition, chemical symbols are drawn with letters $0.07 \mathrm{in}$. high (before projection) and displaced from the atomic centers by $0.18 \mathrm{in}$. horizontally and 0.21 in. vertically. (See 3.3.8.)

1716

0
$0 \quad 0$
.07

2
.18

.21

The hydrogen atoms (ANR $=3,4)$ are drawn with a different standard model (instruction 712) than the carbon atoms. Chemical symbols are $0.07 \mathrm{in}$. high and offset $0.15 \mathrm{in}$. horizontally and 0.13 in. vertically.

$1 \quad 712$

0

0

0

3

.07

.15

.13

The most convenient procedure for drawing bonds is to use the implicit bond instruction 812 (see 3.3.9.2). All other information can be entered with a single Format No. 2 trailer card.

2812

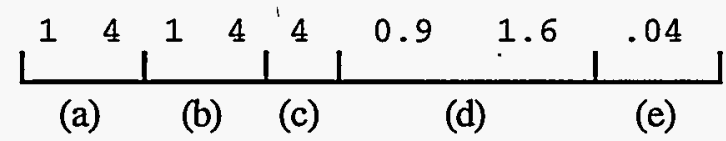

where the parts designate:

(a) origin atom number run of those atoms that must be at one end of each bond,

(b) target atom number run of those atoms that must be at the other end of each bond,

(c) bond type 4,

(d) the distance range, 0.9 to $1.6 \AA$, that will cover all covalent bond distances, and

(e) the bond radius, $0.04 \AA$. not desired.

The remaining fields on the card are blank since a complete set of bond distance labels is 
The last feature of the illustration is the labeling with 900 instructions (see 3.3.10). Because of the symmetry, there are only two different $\mathrm{C}-\mathrm{C}$ bond lengths in cubane. These are $\mathrm{C} 1-\mathrm{C} 1$ and $\mathrm{C} 1-\mathrm{C} 2$. One example of each of these bonds is labeled. For variety, one is labeled with a normal bond-length label and the other with a perspective label. The two bonds that can be labeled most advantageously are 155504-155503 and 255504-155505. The labels will be 0.07 in. high and displaced vertically from the bond center by -0.2 in.

$\begin{array}{llllllll}906 & 155504 & 155503 & 0 & 0 & .07 & 0 & -.2 \\ 916 & 255504 & 155505 & 0 & 0 & .07 & 0 & -.2\end{array}$

Finally, a caption for the illustration is drawn. This can conveniently be positioned by "hanging" it from the.dummy atom 555501 and "bouncing" it 1.8 in. from the left $x$ boundary and 0.3 in. from the lower $y$ boundary. The caption is $0.15 \mathrm{in}$. high.
$3 \quad 902 \quad 555501$
0
1.8
0.3
.15
0
0 CUBANE

The save sequence is now terminated.

1102

The plotting origin is then shifted 2.375 in. along $x$ with a 202 instruction in preparation for the right-eye view.

$202 \quad 2.375$

The stereo rotation of $-2.7^{\circ}$ about axis 2 is now performed for the right-eye view with a 503 instruction. (Note that this rotation starts with the reference orientation, not the previous working orientation.)

503

$$
2 \quad-2.7
$$

The save sequence is now repeated for the right eye. (Note that the ATOMS array contains the same information that it did when the first view was drawn.)

The illustration is now complete. Plotting is terminated with a 202 instruction with no parameters.

202

Finally, a -1 instruction terminates the program. (See 3.3.13.)

$-1$ 


\subsubsection{Illustration of the Example}

Figure 2.1 shows a stereoscopic illustration of cubane exactly as it was produced by ORTEP. The drawing has not been retouched. Note that one bond distance label was drawn in perspective along the bond, and the other was drawn as a regular label parallel to the page.

Note, too, that while most of the atom labels are easy to read and it is clear which atoms they are labeling, some are in locations where the atom being labeled is ambiguous and some are not corrected for overlap. The first problem is the result of labeling a number of atoms with the 700 instruction that draws the atoms. More precise placement can be accomplished by labeling atoms individually (see 7.1) though this can be a time consuming process.

Overlap, particularly for chemical symbols and bond distance labels, is often one of the major problems encountered when producing an illustration. For nonstereoscopic figures, it may be better to add the lettering after the drawing is completed. However, for stereoscopic figures, adding the lettering after the illustration is produced is unsatisfactory because of the necessity for exact relative placement of the lettering on the two views to maintain good stereopsis. In this case, it is best to let ORTEP place the lettering.

The ORTEP input consisting of the crystal data and ORTEP instructions used to produce the structure illustration immediately follows the figure. The example utilizes a wide range of instructions in order to demonstrate their use. As is the case with any programming system, there are many ways of doing a given problem. The user should examine the example carefully since several useful techniques are illustrated. Additional examples are provided in Section 7.

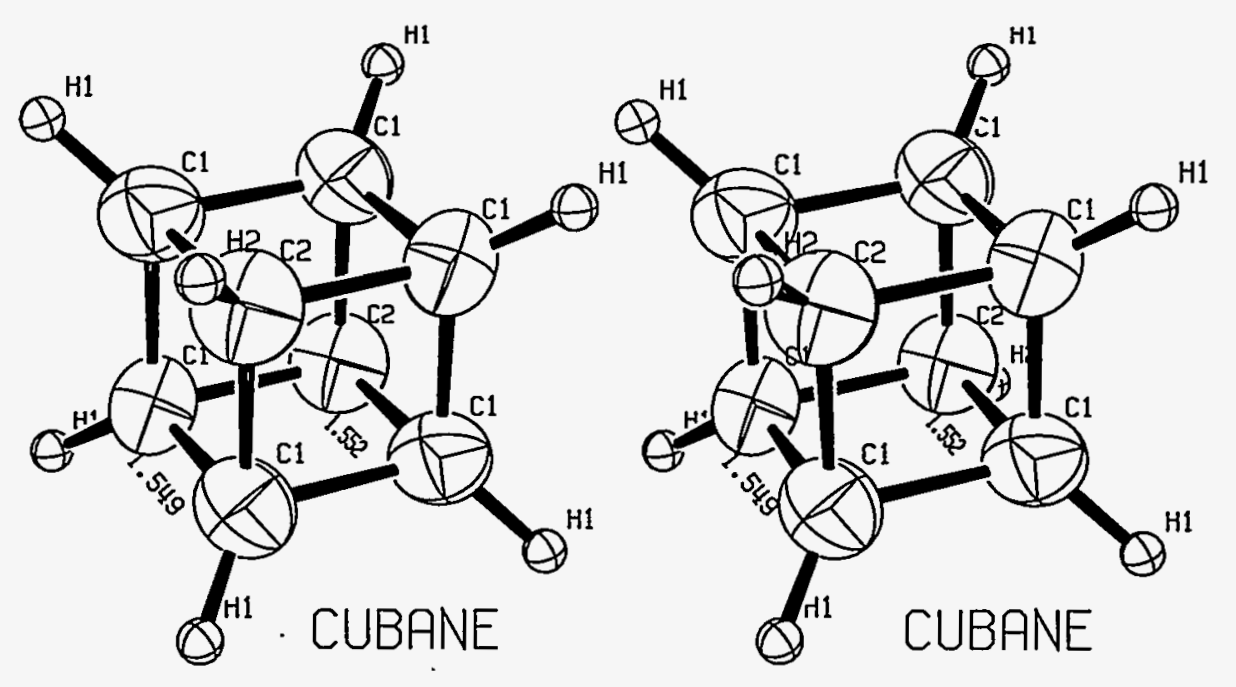

Fig. 2.1. Cubane drawn by ORTEP as a stereoscopic pair. 
CUBANE, E.B. FLEISCHER, J. AM. CHEM. SOC., 86, 3889 (1964)

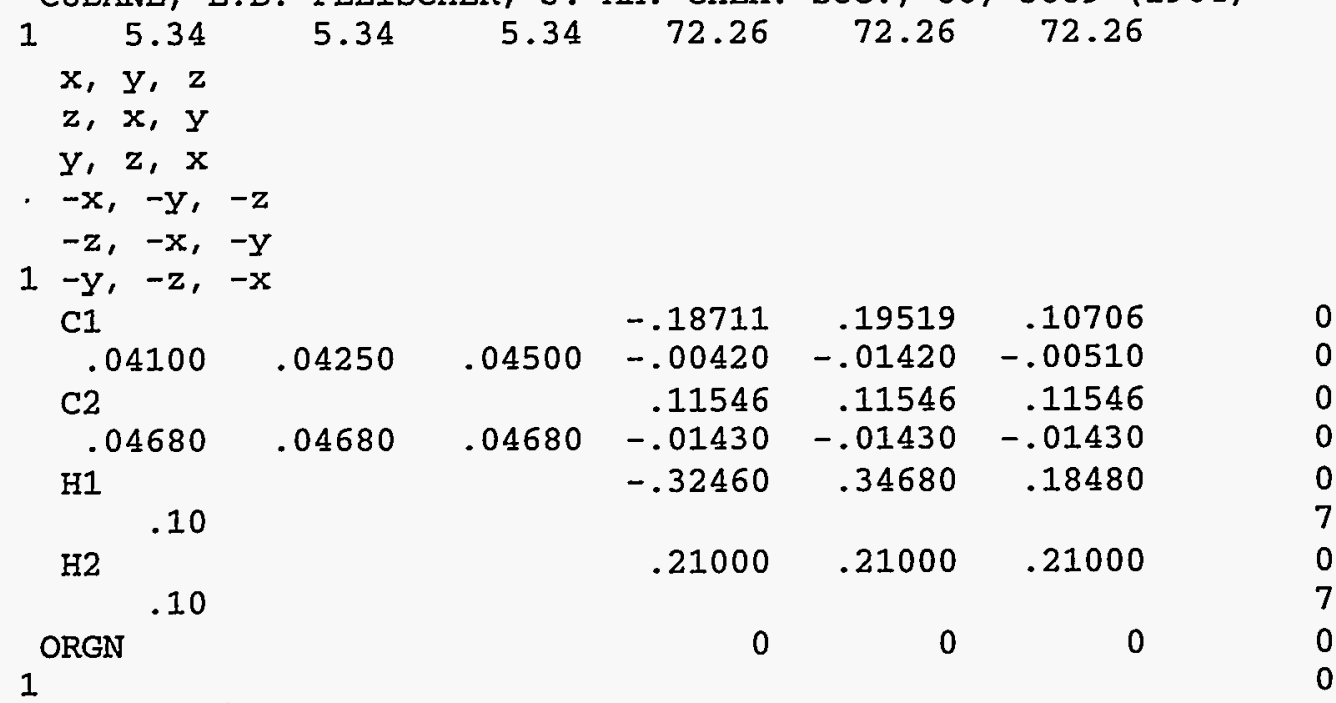

\# FIND ALL NEIGHBOR ATOMS AROUND C1 AND C2 OUT TO 3.61 A $\begin{array}{llllll}101 & 155501 & 2 & 1 & 4 & 3.61\end{array}$

\# FIND COVALENT BONDS AND BOND ANGLES AROUND CARBONS $\begin{array}{llllll}102 & 155501 & 2 & 1 & 4 & 1.8\end{array}$

\# INITIALIZE PLOTTING 201

\# $2.8 \times 2.8$ BOUNDARY, $2.3 \times 2.3$ INSIDE 0.25 MARGIN, VIEW FROM 10 INCHES $\begin{array}{lllll}301 & 2.8 & 2.8 & 10 . & .25\end{array}$

\# ENCLOSER SPHERE OF RADIUS 3.2 A ABOUT DUMMY ATOM 5,555,01 $\begin{array}{llllll}402 & 555501 & 5 & 1 & 4 & 3.2\end{array}$

\# ORIGIN ON DUMMY ATOM 555501, VECTORS ALONG 2 EDGES OF CUBANE $\begin{array}{llllll}501 & 555501 & 255501 & 155501 & 255501 & 155502\end{array}$

\# ROTATE 25 DEGREES ABOUT $Y$, THEN 28 DEGREES ABOUT NEW X $\begin{array}{lllll}502 & 2 & 25 . & 1 & 28 .\end{array}$

\# AUTOMATIC SCALE AND POSITION, 50 PERCENT PROBABILITY ELLIPSOIDS $\begin{array}{lllll}604 & 0 & 0 & 0 & 0\end{array}$

\# STEREO ROTATION OF 2.7 DEGREES ABOUT Y FOR LEFT EYE $\begin{array}{lll}503 & 2 & 2.7\end{array}$

\# START SAVE SEQUENCE 1101

\# STORE PROJECTED ATOMS AND BONDS FOR OVERLAP, MARGIN SET BY DEFAULT 21001

$$
\begin{array}{llllllll}
1 & 4 & 1 & 4 & 4 & 0.9 & 1.6 & .04
\end{array}
$$

\# DRAW CARBON ATOM ELLIPSOIDS, ATOM NUMBER RUN 1-2 1716

$$
12
$$$$
\begin{array}{lll}
.07 & .18 \quad .21
\end{array}
$$

\# DRAW HYDROGEN ATOM SPHERES, ATOM NUMBER RUN 3-4

1712

34

$.07 \quad .15$

\# TYPE 4 BONDS .04 A MEAN RADIUS, ATOMS 1-4 TO ATOMS 1-4, 0.9-1.6 A 2812

$$
\begin{array}{llllllll}
1 & 4 & 1 & 4 & 4 & 0.9 & 1.6 & .04
\end{array}
$$

\# LABEL BOND 155504-155503 WITH REGULAR BOND DISTANCE LABEL $\begin{array}{llllllll}906 & 155504 & 155503 & 0 & 0 & .07 & 0 & -.2\end{array}$

$\begin{array}{ccccccc}\text { \# LABEL BOND } & 255504-155505 & \text { WITH } & \text { PERSPECTIVE } & \text { BOND } & \text { DISTANCE LABEL } & \\ 916 & 255504 & 155505 & 0 & 0 & .07 & 0\end{array}$ 


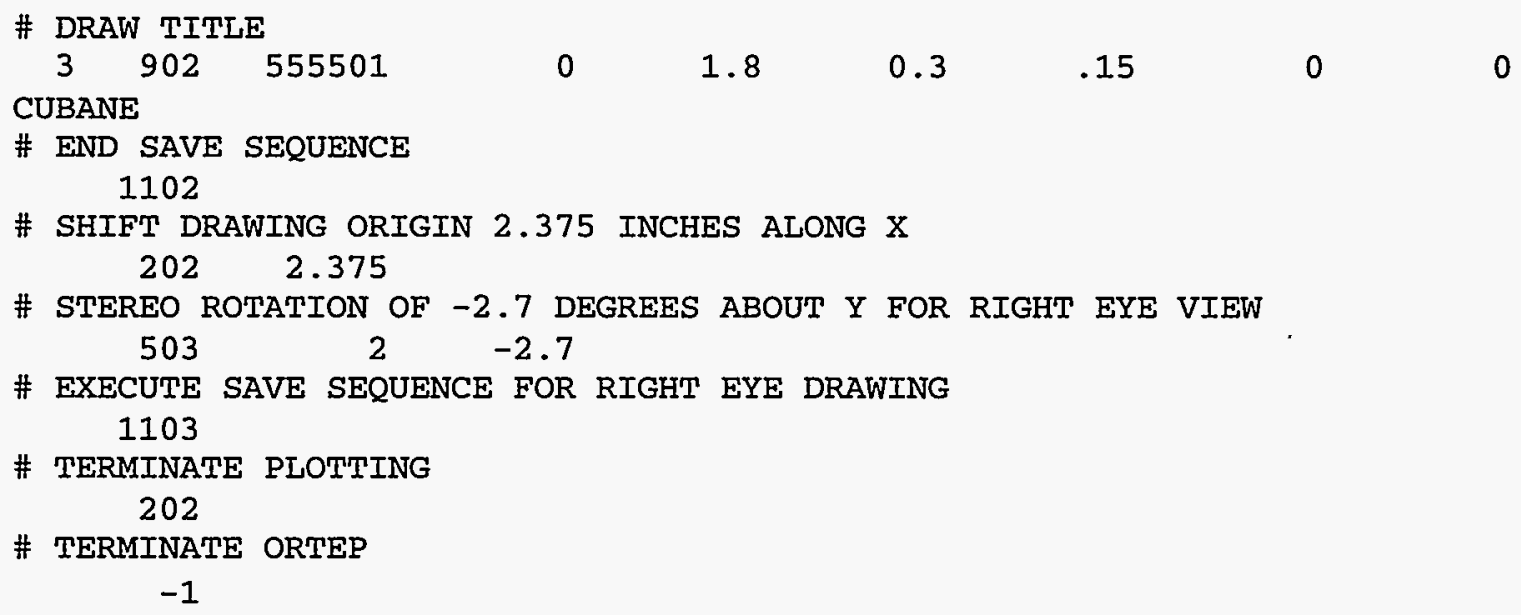




\section{ORTEP INPUT}

\subsection{DEFINITIONS}

Several terms must be defined before the instructions can be explained.

\subsubsection{Atom Designator Code (ADC) and Addressable Point}

A five-component atom designator code is used to specify a particular atom in the crystal within a reasonable distance from the crystallographically defined origin.

$$
\mathrm{ADC}=\mathrm{AN}^{*} 10^{5}+(\mathrm{TA}+5) * 10^{4}+(\mathrm{TB}+5) * 10^{3}+(\mathrm{TC}+5) * 10^{2}+\mathrm{SN},
$$

where

$\mathrm{AN}=$ atom number $(0 \leq \mathrm{AN} \leq \mathrm{NATOM} \leq 500)-$ the numerical position of the atom in the input list of atoms in the asymmetric unit, which contains NATOM atoms. Atom 0 is not in the input atom list but refers to the crystal origin point $(0,0 ., 0$.).

$\mathrm{TA}, \mathrm{TB}, \mathrm{TC}=$ crystal lattice translation digits - translations along cell edges $\mathbf{a}, \mathbf{b}$, and $\mathbf{c}$, respectively. Each digit in an ADC can range from 1 to 9; consequently, it is possible to move up to 4 cells in any direction from the origin cell 555.

$\mathrm{SN}=$ symmetry operator number $(0 \leq \mathrm{SN} \leq \mathrm{NSYM} \leq 96)-$ the numerical position of the symmetry operator in the input list of symmetry operators, which contains NSYM entries. Symmetry operator number 0 is not in the input list but refers to an identity operator. However, the identity operation (corresponding to position $x, y, z)$ generally must be somewhere in the input symmetry operator list and is usually the first operator.

Example: An atom designator code of 347502 refers to atom 3 moved through symmetry operation 2 , then translated -1 cell translation along $\mathbf{a},+2$ cell translations along $\mathbf{b}$, and 0 cell translations along $\mathbf{c}$.

An addressable point in the crystal is any point for which an atom designator code exists. In general, the addressable region is approximately a $9 \times 9 \times 9$ block of unit cells.

\subsubsection{Vector Designator Code (VDC)}

A vector designator code defines a vector using two atom designator codes. The vector direction is from the first to the second.

Example: 253704263704 is a vector along the positive a direction of the crystal lattice.

\subsubsection{Atom Designator Run (ADR)}

An atom designator run is a straight run sequence of atoms that is defined using two atom designator codes with a "-" preceding the second of the two. The run hierarchy is: first, atom number $\mathrm{AN}$; second, symmetry operator number $\mathrm{SN}$; third, a translation $\mathrm{TA}$; fourth, $\mathbf{b}$ translation TB; and last, $\mathbf{c}$ translation TC. 
Example: ADR (145502-245603) will generate the 8-atom run 145502, 245502, 145503, 245503, 145602, 245602, 145603, 245603.

The following exceptions are allowed in Org. ADRs of instructions 101, 102, 402/412, 403/413, and 404/414 only:

- The "-" may be omitted from the second ADC.

- If the second atom in the atom designator run has the same symmetry and translation components as the first atom, the second atom may be represented by its atom number component alone.

- If the symmetry and translation components of both atoms are 55501, both atoms may be represented by their atom number components alone.

Example: ADR (345502-745502) may also be represented as (345502 745502) or (345502 7). ADR (355501-755501) may also be represented as (355501 755501) or (3555017) or (3 7).

\subsubsection{Atom Number Run (ANR)}

An atom number run is a subset of the atom designator run in which only the atom number AN changes. Normally, an ANR is entered by using only the atom number values for the first and last members of the sequence without a "-".

Example: (1 4) will designate atoms 1, 2, 3, and 4 of the input list.

\subsubsection{Vector Search Code (VSC)}

A vector search code consists of two number runs and a distance range. It is used for finding interatomic distances that have a particular chemical significance, such as covalent and coordination bonds.

Example: Suppose that metal atoms are numbers 1 and 2 in the atom list, oxygen atoms are 6-12, and the interatomic distance range between metals and oxygens is $1.9 \AA$ to $2.4 \AA$. The metal-to-oxygen vectors can be specified by the vector search code (1 2) (6 12) (1.92.4). Several variations of this basic code are used in ORTEP.

The vector searches in ORTEP-III for the 100 instructions and 400 instructions have been generalized to allow both atom number runs and feature number runs (see 3.1.11) with the number run type (see 3.1.12) specified in column 24. In addition, it is no longer necessary to specify all three screening ranges found on this card (i.e., origin, target, and distance range). Any ranges not specified (i.e., zero or blank in the maximum value entry) are omitted from the screening. If there is more than one vector search card for an instruction, the parameter LOGC controls the logic of the screening. If the value is " 0 " (the default), an atom satisfying the screening conditions on any one of the No. 2 trailer cards will be retained (i.e., it is a logical union of the results). If the value is " 1 ", an atom must satisfy the screening conditions on all the cards to be retained (i.e., it is an intersection of the resuits). If a value of " 1 " is needed for LOGC, a " 1 " is placed in column 27 of a Format No. 1 trailer card that goes between the main instruction card and the Format No. 2 VSC cards. If the value for LOGC is " 0 ", the Format No. 1 card is not needed. 


\subsubsection{Sphere of Enclosure}

A sphere of enclosure specifies some or all of the atoms lying within a sphere of radius $D_{\max }$ about a given "origin" atom without the necessity of delineating each atom individually. The sphere of enclosure is said to contain a complete population if all addressable atoms within the $D_{\max }$ radius are included. If the sphere of enclosure contains only certain types of atoms that are derived from a group of sequential atoms in the input list or atoms having particular features, then the sphere is said to have a partial population. Finally, the population (complete or partial) of the sphere of enclosure can be screened as selectively as desired through the use of vector search codes (see 3.1.5), and the resulting content is called a vector screened population.

A sphere of enclosure can be centered on any addressable atom, but the origin atom should not be chosen in the outermost cells because of the possibility of having nonaddressable points within the $D_{\max }$ radius.

\subsubsection{Box of Enclosure}

A box of enclosure is a parallelepiped that can be centered about any addressable point and assigned arbitrary orientation and dimensions. The orientation depends upon either the unit cell axes (triclinic box of enclosure) or the reference axes (rectangular box of enclosure). The box of enclosure can have a complete population, a partial population, or a vector screened population as described for the sphere of enclosure (see 3.1.6).

\subsubsection{Reference, Working, and Standard Cartesian Coordinate Systems}

Many of the ORTEP calculations use fractional coordinates based on the crystal axes a, $\mathbf{b}$, and $\mathbf{c}$ (triclinic coordinate system); but other steps necessitate the introduction of orthonormal base vector triplets (Cartesian coordinate systems). Two Cartesian systems-reference and working-are utilized. The reference (major) system is used for all operations except plotting, where the working (minor) system is used. For a right-eye or left-eye stereo view, the working system is moved from the reference system by rotation about an axis of the reference system. However, certain decisions made while plotting must still be referred to the reference system to maintain accurate stereopsis. The user can define and orient the two Cartesian systems through the series 500 instructionș. Until a 500-series instruction is given, a "standard Cartesian system" is utilized for both the reference and working systems. The orthonormal base vectors of the standard system are oriented as follows:

$$
\begin{aligned}
& x \text { axis along } \mathbf{a}, \\
& y \text { axis along }(\mathbf{a} \times \mathbf{b}) \times \mathbf{a}, \\
& z \text { axis along }(\mathbf{a} \times \mathbf{b})=\mathbf{c}^{*},
\end{aligned}
$$

where $\mathbf{a}, \mathbf{b}$, and $\mathbf{c}$ are crystal axes and $\times$ denotes the outer vector product (cross product). The symbol $c^{*}$ refers to a reciprocal axis.

\subsubsection{Prime Parameters and Primer Constants}

The more basic among the many settable parameters in ORTEP gre the prime parameters. The default values assigned to these prime parameters are often similar or identical from one problem to the next. Among the first things ORTEP does is a call to subroutine PRIME, which sets as many prime parameters as possible to reasonable default primer constant values. For 
example, the maximum plot dimensions (instruction 301) are set to $10.5 \mathrm{in}$. for $x_{\max }$ and $8.0 \mathrm{in}$. for $y_{\max }$, and the overall scale for plotting (instruction 600 series) is set to $1.0 \mathrm{in} . / \mathrm{A}$. If the value assigned to a particular constant by the PRIME subroutine is satisfactory, the user does not have to change the value with ORTEP instructions.

\subsubsection{Atom Feature}

An atom feature is a user-defined characteristic of a group of atoms that may be assigned to the atoms in the ORTEP input as needed for a given task. For example, the atomic number may be provided with each atom to make selecting atoms of the same element easier. Up to two features may be assigned to each atom. These are referred to as Feature \#1 and Feature \#2. (See 4.6.)

\subsubsection{Feature Number Run (FNR)}

A feature number run is used to identify those atoms having a particular atom feature within a specified value range.

\subsubsection{Number Run (NR) and Number Run Type}

A number run is a generic term that refers to both atom number runs (ANR) (see 3.1.4) and feature number runs (FNR) (see 3.1.11). The number run type identifies the number run. Number run type 0 refers to an ANR, type 1 refers to an FNR on Feature \#1, and type 2 refers to an FNR on Feature \#2.

\subsection{CRYSTAL STRUCTURE DATA INPUT}

\subsubsection{Title}

The first card in the ORTEP input is a title card with FORMAT (18A4), consisting of up to 72 characters of alphanumeric identification information. This will appear periodically in the output file.

\subsubsection{Cell Parameters}

The second input card contains the cell parameters with FORMAT (I1,F8.6,5F9:6). Any one of the following four input alternatives may be used.

\begin{tabular}{|c|c|c|c|c|}
\hline Columns & Type A & Type B & Type C & Type D \\
\hline 1 & $\begin{array}{c}\text { Symmetry } \\
\text { format indicator }\end{array}$ & $\begin{array}{l}\text { Symmetry } \\
\text { format indicator }\end{array}$ & $\begin{array}{c}\text { Symmetry } \\
\text { format indicator }\end{array}$ & $\begin{array}{c}\text { Symmetry } \\
\text { format indicator }\end{array}$ \\
\hline $2-9$ & $\mathbf{a}(\AA)$ & $\mathbf{a}(\AA)$ & $\mathbf{a}^{*}\left(\AA^{-1}\right)$ & $\mathbf{a}^{*}\left(\AA^{-1}\right)$ \\
\hline $10-18$ & $\mathbf{b}(\AA)$ & $\mathbf{b}(\AA)$ & $\mathbf{b}^{*}\left(\AA^{-1}\right)$ & $b^{*}\left(\AA^{-1}\right)$ \\
\hline $19-27$ & c $(\AA)$ & c $(\AA)$ & $c^{*}\left(\AA^{-1}\right)$ & $c^{*}\left(\AA^{-1}\right)$ \\
\hline $28-36$ & $\alpha\left(^{\circ}\right)$ & $\cos \alpha$ & $\alpha^{*}\left({ }^{\circ}\right)$ & $\cos \alpha^{*}$ \\
\hline $37-45$ & $\beta\left(^{\circ}\right)$ & $\cos \beta$ & $\beta^{*}\left({ }^{\circ}\right)$ & $\cos \beta^{*}$ \\
\hline $46-54$ & $\gamma\left({ }^{\circ}\right)$ & $\cos \gamma$ & $\gamma^{*}\left({ }^{\circ}\right)$ & $\cos \gamma^{*}$ \\
\hline
\end{tabular}


No indicator is needed to specify the input type. The routine assumes that $\mathbf{a} \geq 1.0 \AA, \mathbf{a} *<1.0 \AA^{-1}$, $\alpha\left(\right.$ or $\left.\alpha^{*}\right) \geq 1.0^{\circ}$, and $|\cos \alpha|\left(\right.$ or $\left.\mid \cos \alpha^{*}\right)<1.0$. The parameters $\mathbf{a}^{*}$, etc., refer to the reciprocal unit cell such that $a^{*} \mathbf{a}^{*}=1$. All four types will be printed in the output regardless of which type was used for input.

An integer value in column 1 of the cell parameter card indicates the format used for the crystal symmetry cards that follow.

\subsubsection{Symmetry}

Crystal symmetry in ORTEP-III may be supplied in either of two styles. The first of these is identical to that of OR TEP- $\Pi^{2}$ and is triggered by having a " 0 " or blank in column 1 of the cell parameter card. A " 1 " in that position indicates the symmetry operators are provided in a free format using the xyz coordinate triplet notation found in the International Tables for Crystallography. ${ }^{4}$ These two styles are referred to as Type 0 and Type 1 , respectively.

The number of symmetry cards (NSYM) may not exceed 96 . At least one (the identity operator) is required. The reason for the maximum of 96 is that the symmetry operator number (SN) occupies only two places in the $\mathrm{ADC}$ (see Sect. 3.1.1). If it is not possible to supply all the symmetry operators for the space group (or if the user chooses not to supply all of them), each unique atom in the ORTEP input file will require multiple entries with those lattice centering translations added that are not provided in the symmetry cards. (See 7.5 for an example.)

A Type 0 symmetry card has FORMAT (I1,F14.10,3F3.0,2(F15.10,3F3.0)) and will be interpreted in one of two ways, depending on the value of the number in columns 70-72. If that number is $<5.0$, the card is interpreted as a crystallographic symmetry operation; but if the number is $\geq 5.0$, the card is interpreted as a general helix-screw symmetry operation ${ }^{\dagger}$ along the $c^{*}$ crystal axis (third axis of the standard Cartesian system; see 3.1.8). The two symmetry types can be intermixed if desired.

tThe general helix-screw symmetry operation is not an allowed element of a crystallographic group, so the molecular environment of the transformed unit will not in general be identical to that of the untransformed unit (unless the crystal is considered to be one dimensional). This input is simply an expedient for use in plotting helical polymer structure models with minimum input. In general, it would be possible to produce the same results by specifying the complete crystallographic asymmetric unit and normal crystallographic symmetry transformations.

This input mode is only meaningful if the cell angles $\alpha$ and $\beta$ are $90^{\circ}$, so that $\mathrm{c}$ lies along $c^{*}$ and the helix can continue uninterrupted from cell to cell along the $\mathrm{c}$ axis. 


\begin{tabular}{|c|c|c|}
\hline Columns & $\begin{array}{l}\text { (a) Crystallographic symmetry } \\
(70-72<5)\end{array}$ & $\begin{array}{l}\text { (b) Helix symmetry } \\
(70-72 \geq 5)\end{array}$ \\
\hline 1 & $\neq 0$ last card only & $\neq 0$ last card only \\
\hline $2-15$ & $T_{1}$ & $T_{1}$ \\
\hline $16-18$ & $S_{11}$ & 一 \\
\hline $19-21$ & $S_{12}$ & - \\
\hline $22-24$ & $S_{13}$ & 一 \\
\hline $25-39$ & $T_{2}$ & $T_{2}$ \\
\hline $40-42$ & $S_{21}$ & - \\
\hline $43-45$ & $S_{22}$ & - \\
\hline $46-48$ & $S_{23}$ & - \\
\hline $49-63$ & $T_{3}$ & $T_{3}$ \\
\hline $64-66$ & $S_{31}$ & $L$ \\
\hline $67-69$ & $S_{32}$ & $M$ \\
\hline $70-72$ & $S_{33}$ & $N$ \\
\hline
\end{tabular}

(a) Crystallographic symmetry: Transformed triclinic coordinates $\left(X_{1}, Y_{1}, Z_{1}\right)$ are obtained from input triclinic coordinates $(X, Y, Z)$ by

or in matrix notation

$$
\begin{aligned}
& X_{1}=T_{1}+S_{11} X+S_{12} Y+S_{13} Z \\
& Y_{1}=T_{2}+S_{21} X+S_{22} Y+S_{23} Z \\
& Z_{1}=T_{3}+S_{13} X+S_{32} Y+S_{33} Z
\end{aligned}
$$

$$
\mathbf{X}_{1}=\mathbf{T}+\mathbf{S X}
$$

where $\mathbf{T}=\left(T_{1}, T_{2}, T_{3}\right)$ as fractions of cell edges.

Only symmetry cards for general symmetry equivalent positions are permitted. Symmetry cards that explicitly designate special positions such as $X, X, X ; X, X, Z ; X, Y, 0 ; 1 / 4, Y, 0 ;$ and $1 / 4,3 / 4,0$ are not allowed.

(b) Helix screw symmetry:

$$
\mathbf{X}_{1}=\mathbf{T}+\mathbf{S X}
$$

where $\mathbf{T}=\left(T_{1}, T_{2}, T_{3}+L^{\prime} N\right)$ as fractions of cell edges and $\mathbf{S}$ is a counterclockwise rotation of $L \cdot M / N$ cycles about the $\mathrm{c}^{*}$ axis.

Example: The Pauling and Corey right-handed alpha helix of poly-L-alanine repeats after 13 turns and 47 residues and can be represented by 47 symmetry cards with $N=47 ; M=13 ; L=0,1, \ldots, 46 ; T_{1}, T_{2}, T_{3}=0$. The input atom list then contains the contents of one residue. (See 7.2.) 
Type 1 crystallographic symmetry cards do not have a specific format with the following two exceptions: (1) the symmetry information on each card must not go beyond column 72 , and (2) column 1 must be " 0 " (or blank) on all symmetry cards other than the last one in the set, which must be non-zero. Below is an example set of Type 1 symmetry cards to illustrate the flexibility of this style.

$$
\begin{aligned}
& X, Y, Z \\
& X \quad-Y \quad Z+1 / 2 \\
& X+0.5, \quad Y+.5, \quad Z \\
& 1 X+1 / 2,-y+1 / 2,1 / 2+z
\end{aligned}
$$

As shown, letters may be either upper or lower case. Commas or blanks may be used to separate the components of the triplet. The three components may not have blanks within them. Decimal fractions may be used with or without an initial 0 . Fractions may precede or follow the letters.

Regardless of how the symmetry information is provided, the last card of the set must have a non-zero value in column 1 to signal the end of the symmetry cards. If the value is " 1 ", the atom parameter information immediately follows in the ORTEP input file as described in Section 3.2.4. If the value is " 2 ", the atom parameter information is read from a different file (see 4.5), and the ORTEP instructions follow the symmetry cards.

\subsubsection{Atom Parameters}

Two cards are required for each input atom. The first contains the chemical symbol, positional parameters, and feature information, if needed, The second contains temperature factor information or other information that specifies how the atom is to be represented on the drawing. Several alternate inputs are possible for each of the two cards, and the number in column 63 denotes the type used on that particular card. The number of atoms (variable NATOM) may range

\begin{tabular}{|c|c|c|c|c|}
\hline Columns & Type 0 & Type 1 & Type 2 & Type 3 \\
\hline $1-6$ & \multicolumn{4}{|c|}{ Up to six alphanumeric characters centered in the six-place field } \\
\hline $7-9$ & \multicolumn{4}{|c|}{ - } \\
\hline $10-18$ & [Feature \#1] & [Feature \#1] & [Feature \#1] & $x_{0}(\AA$, Cartesian $)$ \\
\hline $19-27$ & [Feature \#2] & [Feature \#2] & [Feature \#2] & $y_{0}(\AA$, Cartesian $)$ \\
\hline $28-36$ & $x$ (fractional, crystal) & $x(\AA$, crystal $)$ & $x(\AA ̊$, Cartesian $)$ & $r(\AA$, cylindrical $)$ \\
\hline $37-45$ & $y$ (fractional, crystal) & $y(\AA$, crystal $)$ & $y(\AA$, Cartesian $)$ & $\phi\left({ }^{\circ}\right.$, cylindrical $)$ \\
\hline $46-54$ & $z$ (fractional, crystal) & $z(\AA$, crystal $)$ & $z(\AA$, Cartesian $)$ & $z(\AA$, cylindrical $)$ \\
\hline
\end{tabular}
from 1 to 500 .

\subsubsection{Positional Parameters}

The positional parameter cards have FORMAT (A6,3X,6F9.0). 
Type 0 is the normal input based on triclinic coordinates. Coordinates in Angstroms along the unit cell vectors may be entered with Type 1 . Type 2 may be used to place a model described in Cartesian coordinates onto a general triclinic lattice. The orientation of the Cartesian system $x y z$ in the general lattice abc is the standard type described in Section 3.1.8 with $x$ along a and $z$ along $c^{*}$. Type 3 is similar to Type 2 except that cylindrical coordinates $r, \phi, z$ are used and the axis of the system can be displaced from zero in the $x y$ Cartesian plane by the displacement $x_{0}, y_{0}$. Cylindrical coordinates are often used to describe helical structures. The $x_{0}, y_{0}$ translation should be zero if helical symmetry operators are used. This translation feature is meant to be used for explicitly describing the contents of a multiple helix cell.

Column fields $10-18$ and 19-27 on Type 0,1 , and 2 positional parameter cards may be used in ORTEP-III to enter "feature" information about the atoms (see 3.1.10 and 4.6). Normally, these fields are ignored by ORTEP so any numeric values may be here or the fields may be blank. The information in these fields will be interpreted as atom features only if instructions are invoked that specifically look at atom features. Features cannot be entered on Type 3 positional parameter cards.

\subsubsection{Temperature Factors}

Temperature factor cards have FORMAT (I1,F8.0,5F9.0,7X,F2.0).

\begin{tabular}{|c|c|c|c|c|c|c|}
\hline \multirow{2}{*}{$\begin{array}{c}\text { Columns } \\
1\end{array}$} & $\begin{array}{c}\text { Type } \\
0,1,2,3,10\end{array}$ & $\begin{array}{c}\text { Type } \\
4,5,8,9\end{array}$ & \multicolumn{2}{|c|}{ Type 6} & \multicolumn{2}{|c|}{ Type 7} \\
\hline & \multicolumn{4}{|c|}{ A sentinel $\neq 0$ for last atom only } & & \\
\hline $2-9$ & $b_{11}$ & $U_{11}$ & $B$ & $B$ & $R$ & $R$ \\
\hline $10-18$ & $b_{22}$ & $U_{22}$ & 0 & 0 & 0 & 0 \\
\hline $19-27$ & $b_{33}$ & $U_{33}$ & 0 & $\mathrm{VDC}_{1}$ (from) & 0 & $\mathrm{VDC}_{1}$ (from) \\
\hline $28-36$ & $b_{12}$ & $U_{12}$ & 0 & $\mathrm{VDC}_{1}$ (to) & 0 & $\mathrm{VDC}_{1}$ (to) \\
\hline $37-45$ & $b_{13}$ & $U_{13}$ & 0 & {$\left[\mathrm{VDC}_{2}\right.$ (from) } & 0 & {$\left[\mathrm{VDC}_{2}\right.$ (from) } \\
\hline $46-54$ & $b_{23}$ & $U_{23}$ & 0 & $\mathrm{VDC}_{2}$ (to)] & 0 & $\mathrm{VDC}_{2}$ (to)] \\
\hline $55-63$ & $0,1,2,3,10$ & $4,5,8,9$ & $6(\operatorname{or} 0)$ & $6($ or 0$)$ & 7 & 7 \\
\hline
\end{tabular}

Anisotropic temperature factor Types $0,1,2,3$, and 10 use the following formula for the complete temperature factor.

$$
\text { Base }^{-D\left(b_{11} h^{2}+b_{22} k^{2}+b_{33} l^{2}+c b_{12} h k+c b_{13} h l+c b_{23} k l\right)}
$$

The coefficients $b_{i j}(i, j=1,2,3)$ of the various types are defined with the following constant settings.

$$
\begin{array}{ll}
\text { Type 0: } & \text { Base }=e, c=2, D=1 \\
\text { Type 1: } & \text { Base }=e, c=1, D=1 \\
\text { Type 2: } & \text { Base }=2, c=2, D=1 \\
\text { Type 3: } & \text { Base }=2, c=1, D=1 \\
\text { Type 10: } & \text { Base }=e, c=2, D=2 \pi^{2}
\end{array}
$$


Anisotropic temperature factor Types 4,5,8, and 9 use the following formula for the complete temperature factor, in which $\mathbf{a}_{1}^{*}, \mathbf{a}_{2}, \mathbf{a}_{3}$ are reciprocal cell dimensions.

$$
\exp \left[-D\left(\mathbf{a}_{1}^{* 2} U_{11} h^{2}+\mathbf{a}_{2}^{* 2} U_{22} k^{2}+\mathbf{a}_{3}^{* 2} U_{33} l^{2}+C \mathbf{a}_{1}^{*} \mathbf{a}_{2}^{*} U_{12} h k+C \mathbf{a}_{1}^{*} \mathbf{a}_{3}^{*} U_{13} h l+C \mathbf{a}_{2}^{*} \mathbf{a}_{3}^{*} U_{23} k l\right)\right]
$$

The coefficients $U_{i j}(i, j=1,2,3)$ of the various types are defined with the following constant settings.

$$
\begin{array}{ll}
\text { Type 4: } & C=2, D=1 / 4 \\
\text { Type 5: } & C=1, D=1 / 4 \\
\text { Type 8: } & C=2, D=2 \pi^{2} \\
\text { Type 9: } & C=1, D=2 \pi^{2}
\end{array}
$$

Type 6 allows the input of the Debye-Waller isotropic temperature factor $B$, which is used as follows:

$$
\exp \left(-B \sin ^{2} \theta / \lambda^{2}\right)
$$

where $\lambda$ is the wavelength and $\theta$ is the Bragg angle. The parameter $B$ is related to mean-square displacement $\overline{\mu^{2}}$ of the atom from its mean position by the relation

$$
B=8 \pi^{2} \overline{\mu^{2}} \text {. }
$$

When the isotropic temperature factor is used, the atom is represented as an isotropic ellipsoid (sphere) with equal principal axes of length $\mu$. When the field in columns 19-27 is " 0 " or blank, the directions of the principal axes are along the standard Cartesian system axes (see 3.1.8). However, these arbitrary orthogonal vectors can be reoriented by using the two vector designator codes $\mathrm{VDC}_{1}$ and $\mathrm{VDC}_{2}$; then the three new principal-axis vectors will be $\mathrm{VDC}_{1},\left(\mathrm{VDC}_{1} \times\right.$ $\left.\mathrm{VDC}_{2}\right)$, and $\mathrm{VDC}_{1} \times\left(\mathrm{VDC}_{1} \times \mathrm{VDC}_{2}\right)$. This is strictly an artistic feature of no physical significance.

Type 7 allows the input of arbitrary spheres of radius $\bar{\mu}=R$ in Angstroms. The vector triplet orientation is specified as with Type 6 . An additional capability allows a completely blank card (except perhaps column 1) to be used for a temperature factor card. In this case the program assumes Type 7 with an $R=0.1 \AA$.

If $\mathrm{VDC}_{2}$ is omitted on Type 6 or Type 7 temperature factor cards, the program will choose one of the three lattice vectors for $\mathrm{VDC}_{2}$.

A Type 10 temperature factor input card may be used to load Cartesian temperature factors having components in the standard Cartesian system (see 3.1.8). This capability complements the Type 3 Cartesian positional parameter input system (see 3.2.4.1) and is useful for plotting mean-square displacements caused by internal molecular motions as calculated from spectroscopic normal-coordinate analyses.

New in ORTEP-III is the method for specifying the orientations and sizes of the elongated pass (cigar-shaped) and flattened pale (pancake-shaped) ellipsoids used in critical net illustrations (see 7.5) without giving their quadratic form coefficients. The temperature factor card following the atom parameter card for a pass or pale has the format shown below. 


\begin{tabular}{cc} 
Columns & \\
\hline 1 & A sentinel $\neq 0$ for last atom only \\
$2-9$ & Unique axis length $(\AA)$ \\
$10-18$ & Second (and third) axis length $(\AA)$ \\
$19-27$ & $\mathrm{VDC}_{1}$ (from) \\
$28-36$ & $\mathrm{VDC}_{1}$ (to) \\
$37-45$ & {$\left[\mathrm{VDC}_{2}\right.$ (from) } \\
$46-54$ & $\mathrm{VDC}_{2}$ (to)] \\
63 & 7
\end{tabular}

$\mathrm{VDC}_{1}$ is a vector parallel with the unique axis of the cigar-shaped pass or pancakeshaped pale and $\mathrm{VDC}_{2}$ is a second vector not parallel with $\mathrm{VDC}_{1}$ such that $\mathrm{VDC}_{1} \times \mathrm{VDC}_{2}$ is a second principal axis of that ellipsoid. If $\mathrm{VDC}_{1}$ and $\mathrm{VDC}_{2}$ are parallel, $\mathrm{VDC}_{2}$ is replaced by a suitable lattice translation vector. $\mathrm{VDC}_{2}$ may be omitted from the input if desired, and the program will choose one of the three lattice vectors for $\mathrm{VDC}_{2}$.

\subsection{INSTRUCTION INPUT}

The instructions are the commands used in programming an illustration, and there is no required sequence for the instructions, except as indicated by the programming logic.

\subsubsection{Instruction Format}

There are four different formats used for ORTEP instructions, which are numbered 0,1 , 2, and 3. Format No. 0 is always used for a new instruction and includes the instruction number. Some instructions require trailer (continuation) cards, which use Format Nos. 1, 2, and 3. The program is informed what the format of the next card will be with the value in the "look ahead" field, column 3 . The program action is also influenced by this information.

\begin{tabular}{cl}
$\begin{array}{c}\text { "Look ahead" } \\
\text { on current card } \\
\text { (column 3) }\end{array}$ & \multicolumn{1}{c}{ Next card will be } \\
0 or blank & Format No. 0, a new instruction \\
1 & $\begin{array}{l}\text { Format No. 1, continuation of } \\
\text { present instruction }\end{array}$ \\
2 & $\begin{array}{l}\text { Format No. 2, continuation of } \\
\text { present instruction }\end{array}$ \\
3 & $\begin{array}{l}\text { Format No. 3, alphanumeric } \\
\text { information }\end{array}$
\end{tabular}

\section{Program action}

Execute present instruction; then read next new instruction card

Read continuation card and then check its "Look ahead"

Read continuation card and then check its "Look ahead"

Read alphanumeric information and execute instruction; then read next new instruction card 
The Format No. 0 instruction card and the Format No. 1 parameter continuation card have the FORMAT (I3,I6,7F9.0), but the former includes the instruction number while the latter does not. A maximum of 19 Format No. 1 continuation cards per instruction is permitted.

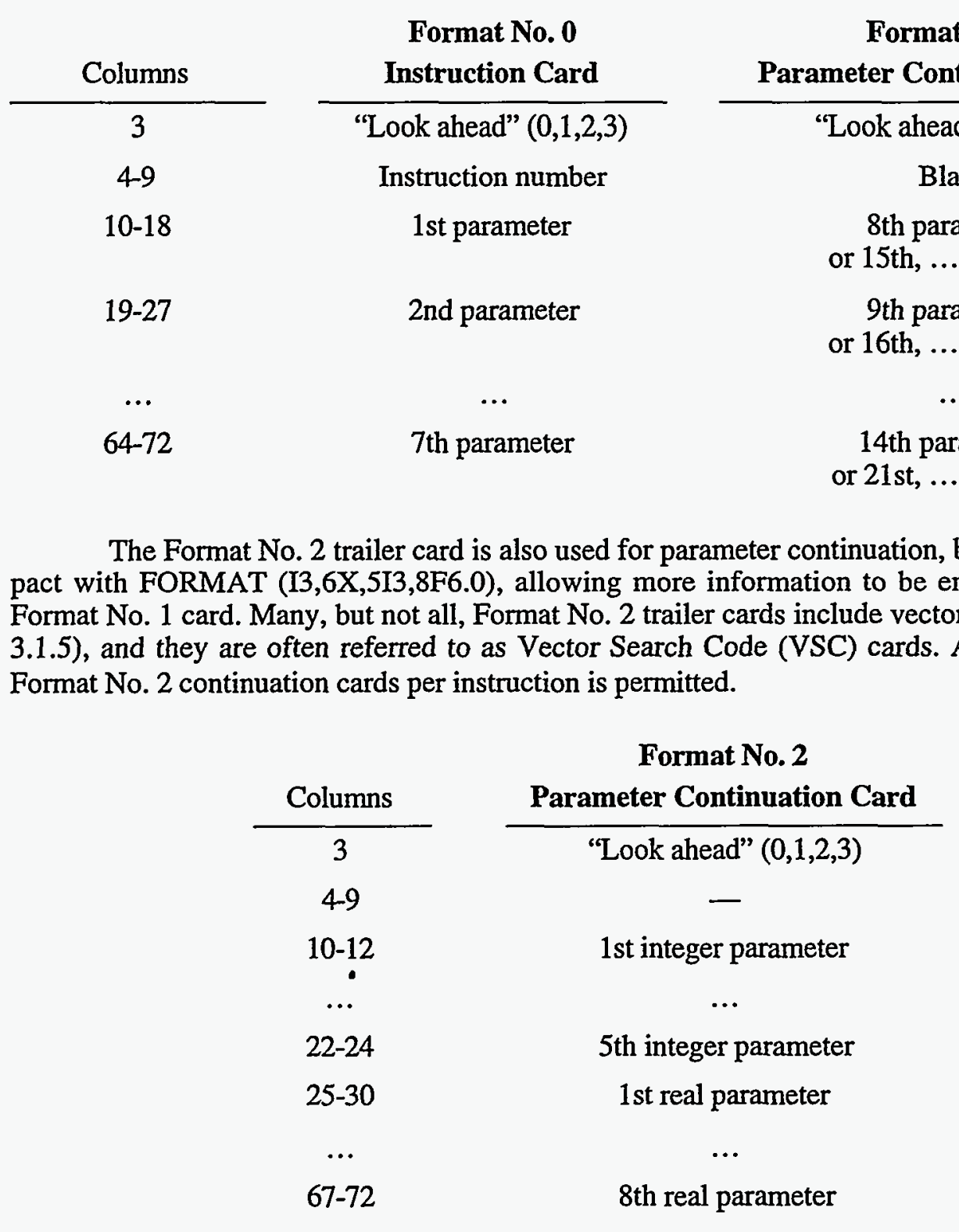

Format No. 3 trailer cards with FORMAT (18A4) are used for entering label information and are required for instructions 902,903 , and 913 . As a trailer to these, they may contain up 72 characters of alphanumeric information in columns 1-72, centered about columns 36 and 37 or beginning in column 1 for automatic centering. These may also be used with other instructions as a device to transfer comments about the particular instruction to the ORTEP output listing. There is no "look ahead" column in Format No. 3; the next card must be a new instruction card. 


\subsubsection{Structure Analysis Instructions (100 Series)}

This series of instructions is not connected with drawing illustrations but rather with obtaining a convenient tabulation of the chemically interesting aspects of a crystal structure, including interatomic distances and angles and principal axes of thermal motion. If the ORTEP output is omitted (see 4.1), these instructions do nothing.

\subsubsection{Instructions 101 and 102}

These instructions call subroutine SEARC, which finds all "target" atoms within a sphere of enclosure of radius $D_{\max }$ about a particular "origin" atom. The instruction card has an atom designator run (see 3.1.3) of one or several origin atoms (Org. ADR) and an atom number run of target atoms (Tar. ANR). The Org. ADR allows calculation of several spheres successively with a printout of results after each one.

Example: Suppose there are nine atoms in the input list and we want to find all atoms surrounding atoms 365502,465502 , and 565502 out to a maximum radius of $4 \AA$.

$$
\begin{array}{llllll}
101 & 365502 & 5 & 1 & 9 & 4.0
\end{array}
$$

The selection of the interatomic vectors from a particular origin atom to the target atoms can be narrowed with a vector search code on a Format No. 2 continuation card (see 3.3.1). The first line of the instruction defines a limiting sphere of vectors, and the VSC finds the subset of vectors that satisfy the additional restriction. (See 3.1.5.)

Example: Suppose we want to limit the results of the search in the preceding example to the shell of atoms that lie 2.0-2.7 $\AA$ from each of the origin atoms.

$\begin{array}{rrrrrrrrrr}2 & 101 & 365502 & & & 5 & 1 & 9 & 4.0\end{array}$

Vectors found about a particular origin atom are stored in a table of dimension 200 sorted on distance. Duplicate vectors (not duplicate distances) are eliminated. If more than 200 acceptable atoms are found about an origin atom, the 200 shortest vectors are saved. At the end of the search about each origin atom, the distances are printed out along with the atom designator codes, chemical symbols, and triclinic crystal coordinates for the origin and target atoms. If the instruction is 102, all possible interatomic angles and interatomic distances for the edges opposite the angles are also calculated and printed for the stored vectors. There will be $n(n-1) / 2$ angles for $n$ vectors. The tabulation of atom designator codes, which is obtained automatically when these instructions are given, is often useful for planning an illustration. 


\begin{tabular}{|c|c|c|c|}
\hline Columns & $\begin{array}{l}\text { Instructions } \\
101 \text { and } 102\end{array}$ & Columns & $\begin{array}{l}\text { Format No. } 2 \\
\text { Vector Search Code } \\
\text { (if used) }\end{array}$ \\
\hline 3 & 0,1, or 2 & 3 & 0 or 2 \\
\hline $7-9$ & 101 or 102 & 4-9 & - \\
\hline $10-18$ & Org. ADR (from) & $10-12$ & [Org. NR (from) \\
\hline $19-27$ & Org. ADR (to) & $13-15$ & Org. NR (to)] \\
\hline $28-36$ & Tar. ANR (from) & $16-18$ & [Tar. NR (from) \\
\hline $37-45$ & Tar. ANR (to) & $19-21$ & Tar. NR (to)] \\
\hline \multirow[t]{9}{*}{$46-54$} & \multirow[t]{4}{*}{$D_{\max }(\AA)$} & 24 & [NR type] \\
\hline & & $25-30$ & {$\left[D_{\min }(\AA)\right.$} \\
\hline & & $31-36$ & $\left.D_{\max }(\AA)\right]$ \\
\hline & & $\begin{array}{c}\text { Format No. } 1 \\
\text { Continuation Card }\end{array}$ & \\
\hline & Columns & (if needed) & \\
\hline & 3 & 2 & \\
\hline & $7-9$ & - & \\
\hline & $10-18$ & - & \\
\hline & 27 & LOGC & \\
\hline
\end{tabular}

\subsubsection{Instruction 103}

Principal axes of thermal motion (or arbitrary spheres, according to the temperature factor input) for all atoms in the input list are calculated. The printout contains root-mean-square amplitudes of displacement along the principal axes of the trivariate normal probability density function and direction cosines for the principal axes relative to the reference Cartesian base vectors. A symmetric covariance dispersion matrix based on the reference Cartesian system is also printed out. The diagonal elements are the mean-square displacements along the reference Cartesian axes.

\begin{tabular}{|c|c|}
\hline Columns & Instruction 103 \\
\hline $7-9$ & 103 \\
\hline $10-72$ & Blank \\
\hline
\end{tabular}

\subsubsection{Instructions 105 and 106, Convoluting Sphere of Enclosure and Reiterative Convo-} luting Sphere of Enclosure

These instructions utilize the ATOMS array and thus can only be used after one or more atoms have been placed in the table by a $40 n$ instruction (see 3.3.5). The contents of the array are returned to the condition of entry at the conclusion of instructions 105 and 106. 
All atoms in the ATOMS array that have atom or feature numbers within the origin number run (Org. NR) of the instruction are used as origin points. Interatomic distances for all neighboring atoms (whether or not in the ATOMS array) are found out to the specified radius. Vector search codes on Format No. 2 continuation cards may be used for screening if desired. (See 3.1.5.)

Instructions 105 and 106 are similar, except that instruction 106 keeps repeating the "convolution" process until no new atoms with atom numbers within the Org. NR are found. Instruction 106 is useful for molecular structures where the atoms in the input asymmetric unit do not form an intact molecule. In a case of this nature, it is advisable to place a single atom into the ATOMS array with a 401 instruction and let a 106 instruction find the molecule. Care must be taken to specify a $D_{\max }$ that will enclose only bonded atoms. The 106 instruction should not be used for structures forming infinite chains. Instead, a short sequence of 105 instructions can be used for this case.

\begin{tabular}{|c|c|c|}
\hline Columns & $\begin{array}{l}\text { Instructions } \\
105 \text { and } 106\end{array}$ & Columns \\
\hline 3 & 0,1, or 2 & 3 \\
\hline $7-9$ & 105 or 106 & 49 \\
\hline $10-18$ & Org. NR (from) & $10-12$ \\
\hline $19-27$ & Org. NR (to) & $13-15$ \\
\hline $28-36$ & Tar. NR (from) & $16-18$ \\
\hline $37-45$ & Tar. NR (to) & $19-21$ \\
\hline $46-54$ & $D_{\max }(\AA)$ & 24 \\
\hline \multirow[t]{8}{*}{63} & NR type & $25-30$ \\
\hline & & $31-36$ \\
\hline & & $\begin{array}{c}\text { Format No. } 1 \\
\text { Continuation Card }\end{array}$ \\
\hline & Columns & (if needed) \\
\hline & 3 & 2 \\
\hline & $7-9$ & - \\
\hline & 18 & - \\
\hline & 27 & LOGC \\
\hline
\end{tabular}

Format No. 2

Vector Search Code

(if used)

0 or 2

[Org. NR (from)

Org. NR (to)]

[Tar. NR (from)

Tar. NR (to)]

[NR type]

$\left[D_{\min }(\AA)\right.$

$\left.D_{\max }(\AA)\right]$

Format No. 1

Continuation Card

3.3.3 Plotter Control Instructions (200 Series)

The 200 series is a group of instructions that control the device, real or virtual, that receives the information to output the ORTEP illustration. This may be, for example, an actual hardware plotter, a computer monitor, or a file of page description language instructions that will produce a hard copy of the illustration when the file is downloaded to a printer/plotter. Because no single word can describe all of the possibilities, the word "plotter" will be used in this report. 
The 200 instructions control plotter initialization, plot origin, color control, termination, and any other peripheral commands that are required for a particular equipment configuration or plotting package. When the program is modified for a different equipment configuration, the series 200 instructions, which are executed through subroutine F200, should be redefined to suit the user's requirements.

The 201 instruction initializes plotting and must be executed before any plotting can take place. It is a safe policy always to make this the first instruction card. If the 201 and 301 instructions are omitted, all calculations are carried out but no plotting is done. The 202 instruction with no parameters is used to terminate plotting. With parameters, the 202 instruction may be used to shift the plot origin. The 201/202 (with no parameters) form an initialize/terminate pair and must always appear in pairs in the ORTEP input file. The pair may occur more than once (unlike OR TEP- ${ }^{2}$ ). Each call to 201 begins a new plot page.

Instruction 204 is used to change the color of (or plotter pen used for) subsequent plotting. The default value is 0 for black (or pen \#1). The screen and Postscript drivers built into ORTEP-III define color value 2 as red, 3 as green, 4 as blue, 5 as cyan, 6 as magenta, and 7 as yellow. (The value 1 is also black or pen \#1.)

Instruction 205 is used to vary the thickness of subsequently plotted lines. The unit for width is thousandths of an inch, and the default value is 5. For both the 204 and 205 instructions, if columns 10-18 contain " 0 " or are blank, the default settings are restored.

\begin{tabular}{|c|c|c|c|c|c|}
\hline \multirow[t]{2}{*}{ Columns } & \multirow[t]{2}{*}{$\begin{array}{c}\text { Instruction } \\
201\end{array}$} & \multicolumn{2}{|c|}{$\begin{array}{c}\text { Instruction } \\
202\end{array}$} & \multirow[t]{2}{*}{$\begin{array}{c}\text { Instruction } \\
204\end{array}$} & \multirow[t]{2}{*}{$\begin{array}{c}\text { Instruction } \\
205\end{array}$} \\
\hline & & $\begin{array}{l}\text { Shift plot } \\
\text { origin }\end{array}$ & $\begin{array}{l}\text { Terminate } \\
\text { plotting }\end{array}$ & & \\
\hline $7-9$ & 201 & 202 & 202 & 204 & 205 \\
\hline $10-18$ & - & $\begin{array}{l}\text { Shift along } x \\
\text { (in.) }\end{array}$ & - & $\begin{array}{c}\text { Color } \\
\text { (or pen \#) }\end{array}$ & Pen width \\
\hline $19-27$ & - & $\begin{array}{l}\text { Shift along } y \\
\text { (in.) }\end{array}$ & - & - & - \\
\hline
\end{tabular}

\subsubsection{Drawing Parameter Instructions (300 Series)}

This is a set of instructions for specifying the drawing dimensions, viewing distance, general lettering orientation, pen displacement for line retracing, and ellipse smoothness.

\subsubsection{Instruction 301, Drawing Boundaries, Margin, and Viewing Distance}

This instruction defines the maximum $x$ and $y$ dimensions of the drawing and the margin, all in inches. The boundary dimensions must not exceed those allowed by the plotter. The program will prevent the pen from getting closer than $0.1 \mathrm{in}$. to any boundary. The drawing margin is a constant width border inside the entire boundary. When automatic scaling is used (600 series), the center points of the atoms are prevented from falling in the margin; but the atom representation, which has a finite size, may extend into that area. To accommodate this extension into the margin, the margin width should be large when the overall drawing scale and the ellipsoid scale are expected to be large. 
In addition, instruction 301 specifies the perspective viewing distance, in inches, from the plane of the drawing. An entry of " 0 " for view distance is used to indicate an infinite view distance, and the crystal structure is then mapped in parallel projection normal to the drawing board.

\begin{tabular}{|c|c|c|}
\hline Columns & Instruction 301 & Primer Constant \\
\hline $7-9$ & 301 & - \\
\hline $10-18$ & Drawing $x_{\max }$ (in.) & 10.5 \\
\hline $19-27$ & Drawing $y_{\max }$ (in.) & 8.0 \\
\hline $28-36$ & View distance (in.) & 0. (parallel projection) \\
\hline $37-45$ & Margin width (in.) & 0.5 \\
\hline
\end{tabular}

\subsubsection{Instruction 302, Title Rotation}

For regular titles and chemical symbols, the title rotation is specified with instruction 302. The lettering base line for all lettering is rotated counterclockwise by an angle theta, in degrees, from the $x$ axis of the plotter. Although any value is allowed, $0^{\circ}$ and $-90^{\circ}$ are the values most often used so that the finished drawing has either the $y$ plotter axis vertical or the $x$ plotter axis vertical.

\begin{tabular}{|c|c|c|}
\hline Columns & Instruction 302 & Primer Constant \\
\hline $7-9$ & 302 & - \\
\hline $10-18$ & $\theta\left(^{\circ}\right)$ & 0. \\
\hline
\end{tabular}

\subsubsection{Instruction 303, Retrace Displacement}

Instruction 303 directs certain lines to be made heavier than others by retracing over the path several times with slight pen displacements (DISP) from the original path. For example, if DISP is greater than 0 ., the forward half of the principal plane trace of ellipsoids is drawn heavier than the hidden half so the eye does not confuse the two halves. Also, all regular lettering (but not perspective lettering) is gone over four times to give it a boldface appearance. In ORTEP-III, retracing is turned off by default with the primer constant for DISP set to 0 . in. Retracing can be turned on with the 303 instruction, using a value for DISP greater than 0 . ( 0.005 in. usually works well).

\begin{tabular}{|c|c|c|}
\hline Columns & Instruction 303 & Primer Constant \\
\hline $7-9$ & 303 & - \\
\hline $10-18$ & DISP (in.) & 0. \\
\hline
\end{tabular}

\subsubsection{Instruction 304, Ellipse Smoothness}

Instruction 304 may be used to adjust the smoothness of the plotted ellipses as a function of their size. Smoother ellipses require more computational time and produce larger illustration files. (See 5.2.) Smaller smoothness factors produce smoother ellipses, and larger values produce ellipses with more "jaggies". Factors in the range 0.5-3.0 are recommended; the default value is 1. A value of 0 will draw all size ellipses with 128 points, the maximum provided in ORTEP. 


\begin{tabular}{ccc} 
Columns & & Instruction $\mathbf{3 0 4}$ \\
\hline $\begin{array}{c}7-9 \\
10-18\end{array}$ & & 304 \\
Smoothness factor
\end{tabular}

\subsubsection{ATOMS Array Instructions (400 Series)}

This series allows the user to specify which atoms are to be included in the illustration. The atom designators for the chosen atoms are stored in the ATOMS array for future use by other instructions. The ATOMS array as currently dimensioned holds 500 input atoms, but it can be changed by redimensioning the array in COMMON and setting the variable NATOM in subroutine PRIME to the new value.

Groups of atoms are added to or eliminated from the ATOMS array (which is set to zero at the start of the program) with the $40 n$ and $41 n$ series, respectively. The groups can be denoted by atom designator runs (see 3.1.3), spheres of atoms about any center point (see 3.1.6), and boxes of atoms centered on any point (see 3.1.7). Duplicate entries of the same atomic position are prevented by the program. The content of the ATOMS array is printed in the ORTEP output file after each 400 series instruction.

\subsubsection{Instructions 401/411, Atom Designator Run Add/Eliminate}

These instructions may contain: (a) atom designator codes for single atoms; ( $b$ ) atom designator runs for several atoms in a run; $(c)$ blank fields (except between the two entries of a run); and $(d)$ any combinations of $(a),(b)$, and $(c)$. Since up to 19 Format No. 1 continuations are possible per instruction, up to 70 runs can be made per instruction and an unlimited number of instructions can be used. The ATOMS array and overlap calculation, however, will only accept the first 500 atoms.

\begin{tabular}{|c|c|}
\hline Columns & Instructions 401/411 \\
\hline 3 & 0 (or 1 if continued) \\
\hline $7-9$ & 401 or 411 \\
\hline \multicolumn{2}{|l|}{$10-18$} \\
\hline . & As described above \\
\hline $64-72$ & \\
\hline
\end{tabular}

\subsubsection{Instructions 402/412, Sphere of Enclosure Add/Eliminate}

These instructions allow the user to build or modify the ATOMS array by specifying the contents (complete, partial, or vector screened) of a sphere of enclosure (see 3.1.6) about any addressable point. Instruction 402 adds the contents of the spheres to the ATOMS array omitting positional duplications. The 412 instruction eliminates atoms within the spheres from the ATOMS array if they are present in that array. The instructions call subroutine SEARC, and the instruction input details are identical to those of instructions 101 and 102 (see 3.3.2.1) except for the instruction number. In the ORTEP output file, only the ATOMS array atom designator codes are printed and not the coordinates and interatomic distances. If the origin atoms on which the spheres of enclosure are centered are to be saved, the target atom number run (Tar. ANR) must 
contain those atom numbers. Vector search codes on Format No. 2 continuation cards may be used for screening if desired. (See 3.1.5.)

\begin{tabular}{|c|c|c|c|}
\hline Columns & $\begin{array}{c}\text { Instructions } \\
\mathbf{4 0 2 / 4 1 2}\end{array}$ & Columns & $\begin{array}{l}\text { Format No. } 2 \\
\text { Vector Search Code } \\
\quad \text { (if used) }\end{array}$ \\
\hline 3 & 0,1, or 2 & 3 & 0 or 2 \\
\hline $7-9$ & 402 or 412 & 49 & - \\
\hline $10-18$ & Org. ADR (from) & $10-12$ & [Org. NR (from) \\
\hline $19-27$ & Org. ADR (to) & $13-15$ & Org. NR (to)] \\
\hline $28-36$ & Tar. ANR (from) & $16-18$ & [Tar. NR (from) \\
\hline $37-45$ & Tar. ANR (to) & $19-21$ & Tar. NR (to)] \\
\hline \multirow[t]{9}{*}{$46-54$} & $D_{\max }(\AA)$ & 24 & [NR type] \\
\hline & & $25-30$ & {$\left[D_{\min }(\AA)\right.$} \\
\hline & & $31-36$ & $\left.D_{\max }(\AA)\right]$ \\
\hline & & $\begin{array}{c}\text { Format No. } 1 \\
\text { Continuation Card }\end{array}$ & \\
\hline & Columns & (if needed) & \\
\hline & 3 & 2 & $\cdot$ \\
\hline & $7-9$ & - & \\
\hline & $10-18$ & 一 & \\
\hline & 27 & LOGC & \\
\hline
\end{tabular}

\subsubsection{Instructions 403/413 and 404/414, Box of Enclosure Add/Eliminate and Triclinic Box of Enclosure Add/Eliminate}

The 403/413 instructions allow the user to build or modify the ATOMS array by specifying the contents of a box of enclosure (see 3.1.7) about any addressable point (or atom designator run of addressable points). The three axes of the box are parallel to the three base vectors of the reference Cartesian system, and the semidimensions of the box are specified on the instruction card. If an orientation of the box different from the standard orientation (see 3.1.8) is desired, then a 501 or a 502 instruction, or both, should be used before this instruction to reorient the reference Cartesian system. After this instruction has been executed, the reference system can undergo further reorientation as desired for plotting purposes, etc.

The 404/414 instructions are identical to the 403/413 instructions except that the triclinic box of enclosure is bounded by planes parallel to the principal planes of the crystal lattice. The semidimensions $a / 2, b / 2, c / 2$ refer to fractional (triclinic) coordinates. To specify the contents of the conventional unit cell, one would use $a / 2=b / 2=c / 2=.5$, and the Org. ADR would refer to a point in the input atom list at $(.5, .5, .5)$.

As in the case of the $402 / 412$ instruction, the origin atom on which the box is centered will not be included unless the target atom number run includes the origin atom number. Subrou- 
tine SEARC is used by this instruction, and the instruction input details are similar to those of instructions $402 / 412$ except that $D_{\max }$ is replaced by the semidimension $a / 2$ of the box and the following fields on the card are used to specify the other two semidimensions $b / 2$ and $c / 2$. The box dimensions must be chosen carefully so that the ATOMS array does not overflow. Vector search codes on Format No. 2 continuation cards may be used for screening if desired. (See 3.1.5.)

\begin{tabular}{|c|c|c|c|}
\hline Columns & $\begin{array}{c}\text { Instructions } \\
403 / 413 \text { and } 404 / 414\end{array}$ & Columns & $\begin{array}{l}\text { Format No. } 2 \\
\text { Continuation Card } \\
\text { (if used) }\end{array}$ \\
\hline 3 & 0,1, or 2 & 3 & 0 or 2 \\
\hline $7-9$ & $403,404,413$, or 414 & $4-9$ & 一 \\
\hline $10-18$ & Org. ADR (from) & $10-12$ & [Org. NR (from) \\
\hline $19-27$ & Org. ADR (to) & $13-15$ & Org. NR (to)] \\
\hline $28-36$ & Tar. ANR (from) & $16-18$ & [Tar. NR (from) \\
\hline $37-45$ & Tar. ANR (to) & $19-21$ & Tar. NR (to)] \\
\hline $46-54$ & $a / 2$ ( $\AA$ or fraction) & 24 & [NR type] \\
\hline $55-63$ & $b / 2$ ( $\mathrm{A}$ or fraction $)$ & & \\
\hline $64-72$ & $c / 2$ ( $\mathrm{A}$ or fraction) & & \\
\hline
\end{tabular}

Format No. 1

Continuation Card

$\frac{\text { Columns }}{3}$

$7-9$

$10-18$

27 (if needed)

2

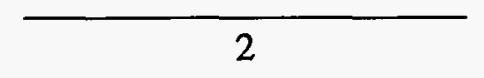

LOGC

3.3.5.4 Instructions 405/415 and 406/416, Convoluting Sphere of Enclosure Add/Eliminate and Reiterative Convoluting Sphere of Enclosure Add/Eliminate

These instructions are used in the same manner as instructions 105 and 106 (see 3.3.2.3). Their function is to add atoms to or eliminate atoms from the ATOMS array. A valid origin atom must be placed in the ATOMS array with a 401, 402, 403, or 404 instruction before these instructions are used. All atoms in the ATOMS array that have atom or feature numbers within the origin number run (Org. NR) of the instruction are used as origin points of convolution. Vector search codes on Format No. 2 continuation cards may be used for screening if desired. (See 3.1.5.)

An example use for the 405 instruction is to complete the coordination shells around metal atoms without having to identify the coordination shell atoms individually. Another use might be to obtain a cluster of atoms out to the $n$th neighbor when only the distance to the first neighbor is known. This can be accomplished by using $n$ consecutive 405 instructions with $D_{\max }$ slightly more than the first neighbor distance. 
Instruction 406 is useful for describing molecular compounds where an unfortunate choice of atoms for the input asymmetric unit does not allow the-molecule to be described by a run. $D_{\max }$ must be chosen judiciously so that the search does not cross molecular boundaries.

Instruction 406 has an added feature that makes it useful for limiting the ATOMS array to those atoms in an asymmetric unit of the unit cell. An example is the examination of electron density maps. When direct methods are used for solving crystal structures, stereoscopic drawings provide a rapid means for screening the $E$ maps. If the interpolated positions of the largest peaks in the Fourier synthesis $E$ map are taken as atom positions, then ORTEP can start at a given peak (usually the largest) and do a reiterative sphere-of-enclosure add to isolate a molecule if one is present. In order to terminate the convolution procedure when extraneous "bridging peaks" link the molecules, a feature has been added to the 406 instruction to prevent multiple instances of an atom (in any of its equivalent positions) from being entered in the ATOMS array. To invoke this feature, a Format No. 1 continuation card with a " 1 " in column 18 (ASYMUNIT) is added to the 406 instruction. The 406 instruction operates in its usual manner if the continuation card is omitted or has a "0" or blank in column 18.

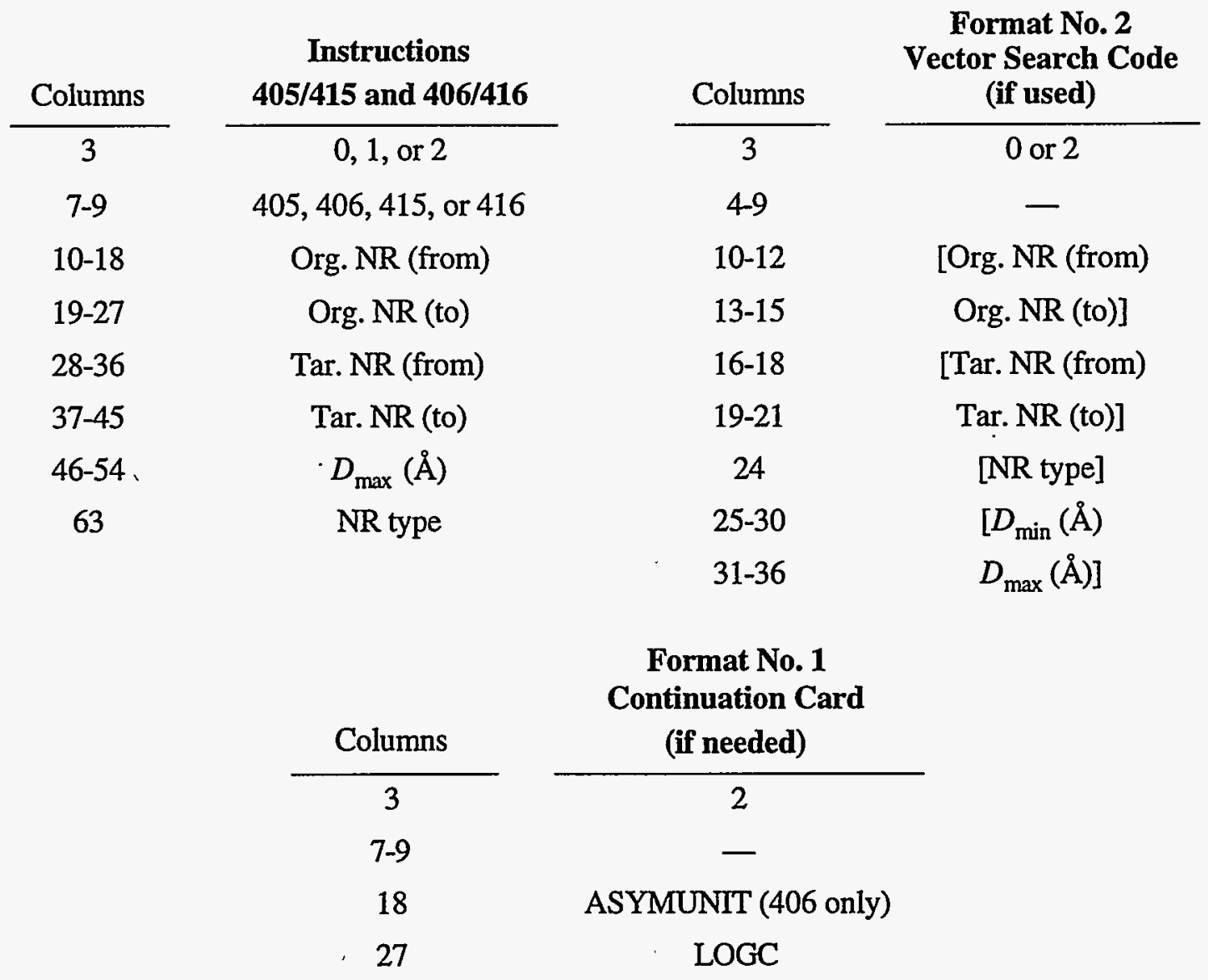




\subsubsection{Instruction 410, Clear ATOMS Array}

This instruction clears the ATOMS array to zero. When the program is first entered, the array is automatically set to zero.

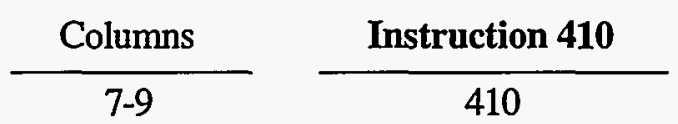

\subsubsection{Orienting Instructions (500 Series)}

For information on instruction 511, see Section 3.3.12.

Any Cartesian coordinate system is based on three orthonormal base vectors and an origin point. In the absence of any 500 instructions, ORTEP calculates the base vectors of the reference and working Cartesian systems (see 3.1.8) from the input cell parameters and sets the origin to $(0 ., 0 ., 0$.$) .$

This series of instructions can be used to reorient the reference and working Cartesian systems. Each time the reference system is redefined or rotated, the working system is automatically made coincident with the reference system. The working system can be displaced from the reference system by rotating about the $x$ or $y$ axis of the reference system with a 503 instruction. The working system is always positioned from the reference system and does not depend on any previous working system orientation. After each 500 series instruction, the base vectors of the relevant Cartesian system are printed in the ORTEP output file. These vectors are based on the triclinic coordinate system. The postfactor transformation matrix for converting from triclinic coordinates to Cartesian coordinates is also printed out. The inverse transformation matrix may be formed by placing the three base vectors together in row vector form.

\subsubsection{Instruction 501, Explicit Reference Cartesian System Assignment}

Instruction 501 allows the user to define the reference Cartesian system explicitly. The origin point in the model (ORGN) is specified with an atom designator code. The three orthonormal base vectors can be described by two non-collinear vectors, and ORTEP provides the two following separate techniques for performing this operation, using vector cross products of the two vectors $\mathbf{u}$ and $\mathbf{v}$. Type 1 produces base vectors that are roughly along the general triclinic coordinate axes of the crystal.

$$
\text { Type } 0 \quad \text { Type } 1
$$

$\begin{array}{lcc}\text { Base vector } 1 \text { ( } x \text { axis }) & \mathbf{u} & \mathbf{u} \\ \text { Base vector } 2(y \text { axis }) & \mathbf{u} \times \mathbf{v} & (\mathbf{u} \times \mathbf{v}) \times \mathbf{u} \\ \text { Base vector } 3(z \text { axis }) & \mathbf{u} \times(\mathbf{u} \times \mathbf{v}) & \mathbf{u} \times \mathbf{v}\end{array}$

The reference system $x$ and $y$ axes will parallel the plotter $x$ and $y$ axes, and the origin point ORGN will lie in the plane of the plotter. The viewer will be looking into the $z$ axis vector of the coordinate system from the viewing distance set by the 301 instruction (see 3.3.4.1) directly above the origin point. 


\begin{tabular}{|c|c|c|}
\hline Columns & Instruction 501 & $\begin{array}{c}\text { Effective } \\
\text { Primer Constant }\end{array}$ \\
\hline $7-9$ & 501 & - \\
\hline $10-18$ & ORGN (ADC) & 000000 \\
\hline $19-27$ & Vector $\mathbf{u}$ (from ADC) & 155501 \\
\hline $28-36$ & Vector $\mathbf{u}$ (to ADC) & 165501 \\
\hline $37-45$ & Vector $\mathbf{v}$ (from ADC) & 155501 \\
\hline $46-54$ & Vector $\mathbf{v}$ (to ADC) & 156501 \\
\hline $55-63$ & 一 & - \\
\hline 72 & Type & 1 \\
\hline
\end{tabular}

\subsubsection{Instruction 502, Reference Cartesian System Rotation}

The crystal model can be given any desired orientation with a series of rotations of the model about the reference system axes. In general, three rotations (e.g., those of an Eulerian system) are sufficient to achieve any orientation, but for convenience an unlimited number of rotations are permitted by ORTEP. In addition, rotations of $120^{\circ}$ about the body diagonal of the reference Cartesian system are permitted (achieved by a cyclic permutation of reference base vectors).

Each operation requires two fields on the instruction card. For axial rotations, the first field of each pair will have the number 1,2 , or 3 to indicate rotation about the $x, y$, or $z$ axis of the reference system, respectively. The second field will have the rotation angle in degrees for a right-handed rotation of the model about the designated axis (i.e., a positive angle signifies a counterclockwise rotation of the structure with the designated axis pointing toward the observer). The body diagonal rotation is designated by either a -1 or a -2 in the first field to indicate a $120^{\circ}$ or a $240^{\circ}$ right-handed rotation about the body diagonal, respectively, and the second field is blank. A -3 would rotate the structure completely around and thus not change its previous orientation. If desired, each rotation can be executed with a separate 502 instruction card.

\begin{tabular}{|c|c|}
\hline Columns & Instruction 502 \\
\hline 3 & 0 (or 1 if continued) \\
\hline $7-9$ & 502 \\
\hline $17-18$ & $1,2,3,-1$, or -2 \\
\hline $19-27$ & $\phi\left(^{\circ}\right)$ (if value in previous field is positive) \\
\hline $28-36$ & \multirow{2}{*}[\ldots]{} \\
\hline $37-45$ & \\
\hline $46-54$ & \multirow{2}{*}[\ldots]{} \\
\hline $55-63$ & \\
\hline
\end{tabular}

\subsubsection{Instruction 503, Working Cartesian System Rotation (Stereoscopic Rotation)}

To define an orientation of the working system that is not coincident with the reference system, a 503 instruction may be used, which allows one rotation about one axis of the reference 
system. Actually any number of successive rotations can be made, but the effect is not cumulative since the starting point for each rotation is always the reference system. Body diagonal rotations are not permitted.

A 503 rotation normally precedes each member of a stereoscopic pair of plots. (See 3.3.6.4 and 5.4.) The rotation is about axis 2 if the stereo pair is to be viewed with the $x$ axis parallel to the observer's interocular line and about axis 1 if the $y$ axis is to be parallel to that line.

\begin{tabular}{|c|c|}
\hline Columns & Instruction 503 \\
\hline $7-9$ & 503 \\
\hline 18 & 1 or 2 \\
\hline $19-27$ & $\omega\left(^{\circ}\right)$ \\
\hline
\end{tabular}

\subsubsection{Instruction 504, Reference Cartesian System Origin Translation}

Instruction 504 is used to translate the origin of the reference Cartesian system along the $x, y$, and $z$ axes of the reference system. Stereo by translation of origin can be achieved with instruction 504, which may be used in place of the 503 instruction. However, the 504 instruction should not be used when the ellipsoids have internal structure because the octants selected for shading may not be the same on both stereo views. (See 5.4.)

\begin{tabular}{|c|c|}
\hline Columns & Instruction 504 \\
\hline $7-9$ & 504 \\
\hline $10-18$ & Translation along $x$ axis (in.) \\
\hline $19-27$ & Translation along $y$ axis (in.) \\
\hline $28-36$ & Translation along $z$ axis (in.) \\
\hline
\end{tabular}

\subsubsection{Instruction 505, Reference Cartesian System Origin at Centroid}

This instruction finds the first moment (i.e., centroid or center of gravity) of the atoms in the ATOMS array and makes this point the origin point (ORGN) of the reference and working coordinate systems. The base vectors of the coordinate systems are unchanged from their previous values. A weighting scheme and screening may be applied to the atoms used to calculate the centroid by using Format No. 2 trailer cards. If no trailer cards are used, all atom positions are entered with unit weights.

\begin{tabular}{cccccc} 
Columns & Instruction 505 & & Columns & & $\begin{array}{c}\text { Continuation Card } \\
\text { (if used) }\end{array}$ \\
\cline { 1 - 1 } \cline { 5 - 6 } & 0 or 2 & & 3 & & or 2 \\
$7-9$ & 505 & & $10-12$ & & NR (from) \\
& & $13-15$ & & NR (to) \\
& & 24 & & NR type \\
& & $25-30$ & & Weight
\end{tabular}




\subsubsection{Instruction 506, Reference Cartesian System Origin at Centroid and Inertial Axis Coordinate System}

The calculation described for instruction 505 is performed; then the second moment matrix about the centroid is calculated, and the reference and working coordinate systems are set up along the principal axes of this matrix. This principal axis system of coordinates is along the inertial axes of the configuration of atoms in the ATOMS array. The $x$ axis is along the long axis of the configuration (i.e., the minimal axis of inertia) and the $z$ axis is along the short axis of the configuration (i.e. the maximal axis of inertia). The overlap along the $z$-view direction is often minimized by this option. Furthermore, the $x y$ plane is the least-squares best plane for the atomic configuration. Format No. 2 trailer cards may be used to supply weights and screening.

\begin{tabular}{|c|c|c|c|}
\hline Columns & $\begin{array}{c}\text { Instruction } \\
506\end{array}$ & Columns & $\begin{array}{c}\text { Format No. } 2 \\
\text { Continuation Card } \\
\text { (if used) }\end{array}$ \\
\hline 3 & 0 or 2 & 3 & 0 or 2 \\
\hline \multirow[t]{5}{*}{$7-9$} & 506 & $4-9$ & - \\
\hline & & $10-12$ & NR (from) \\
\hline & & $13-15$ & NR (to) \\
\hline & & 24 & NR type \\
\hline & & $25-30$ & Weight \\
\hline
\end{tabular}

\subsubsection{Positioning and Scaling Instructions (600 Series)}

These instructions are used to direct the placement of the origin point ORGN (specified by instruction 501, 505, or 506) onto the drawing (dimensioned by instruction 301). In addition the three-dimensional assembly of atoms (chosen by the 400 series instruction) constituting the model is scaled. The atomic centers of the model will then be hanging in space above and below the drawing board, correctly positioned to be projected from the eye point described with instruction 301 .

A second scaling parameter SCAL2 scales the ellipsoid (or sphere) size. It is a dimensionless scale factor ratio used to modify all rms displacement values before plotting equiprobability ellipsoids or spheres. A listing of SCAL2 values vs. probability is given in Table 6.1. The primer constant for SCAL2 is 1.54 , corresponding to $50 \%$ probability. ORTEP-III saves the user the time of looking up the SCAL2 value corresponding to a desired probability. If the value entered in columns 37.45 is a negative whole number in the range -1 to -99 , it is interpreted as the probability of the ellipsoids or spheres, and the value of SCAL2 is set by the program by table lookup, using the values in Table 6.1.

\subsubsection{Normal Modes of Positioning and Scaling}

Several normal modes of operation are available to the user for positioning and scaling the model. Instruction 601 requires the user to supply a complete explicit description of position $(\mathrm{X} 0, \mathrm{YO})$ and scale (SCAL1). At the other extreme, instruction 604 automatically scales and positions the model so that the peripheral projected atom centers will touch two opposite borders and the peripheral atoms in the remaining dimensions will be centered on the drawing. An intermediate mode is available through 602 , which provides automatic scaling after explicit positioning. 
This usually allows only one edge of the model to touch a border. Finally, instruction 603 requires an explicit scale and does automatic centering. In general, the 604 instruction is the easiest and safest one to use, but situations arise in which the user should not relinquish control to the program.

If the instruction's entry for X0, Y0, SCAL1, or SCAL2 is " 0 " or blank, the primer constant value is used. This means that an $\mathrm{X} 0$ or $\mathrm{Y} 0$ cannot be entered as exactly zero. If zero is wanted, a small nonzero number should be entered.

\begin{tabular}{|c|c|c|c|c|c|}
\hline Columns & $\begin{array}{l}\text { Instruc- } \\
\text { tion } 601\end{array}$ & $\begin{array}{l}\text { Instruc- } \\
\text { tion } 602\end{array}$ & $\begin{array}{l}\text { Instruc- } \\
\text { tion } 603\end{array}$ & $\begin{array}{l}\text { Instruc- } \\
\text { tion } 604\end{array}$ & $\begin{array}{l}\text { Primer } \\
\text { Constant }\end{array}$ \\
\hline $7-9$ & 601 & 602 & 603 & 604 & - \\
\hline $10-18$ & X0 & $\mathrm{X} 0$ & - & 一 & 8.5 \\
\hline $19-27$ & Y0 & Y0 & - & - & 5.5 \\
\hline $28-36$ & SCAL1 & - & SCAL1 & - & 1.0 \\
\hline $37-45$ & $\begin{array}{l}\text { SCAL2 or } \\
\text { probability }\end{array}$ & $\begin{array}{l}\text { SCAL2 or } \\
\text { probability }\end{array}$ & $\begin{array}{l}\text { SCAL2 or } \\
\text { probability }\end{array}$ & $\begin{array}{l}\text { SCAL } 2 \text { or } \\
\text { probability }\end{array}$ & 1.54 \\
\hline
\end{tabular}

\subsubsection{Incremental Modes of Positioning and Scaling}

Additional flexibility is provided through the incremental instructions 611,612 , and 613 . These allow the user to "nudge" the model or modify the scale factor SCAL1, or both, after the parameters have been initially set with a previous 600 series instruction. The 611 instruction adds $\triangle \mathrm{X} 0, \Delta \mathrm{YO}$ to the previous $\mathrm{X} 0, \mathrm{Y} 0$ position for the ORGN placement and multiplies the existing SCAL1 by $\triangle S C A L 1$ (except if $\triangle S C A L 1=0$, SCAL1 is unmodified). Instruction 612 increments the position and then does an automatic scaling; 613 first increments the scale (by multiplying by $\triangle S C A L 1)$ and then automatically repositions.

A conservative general approach is to follow a 604 with a 611 having $\triangle \mathrm{XO}=0, \Delta \mathrm{Y} 0=0$ and $\triangle \mathrm{SCAL} 1=0.9$. This will simply reduce the scale $10 \%$ about the origin so that there is more space for labels, etc.

\begin{tabular}{|c|c|c|c|}
\hline Columns & Instruction 611 & Instruction 612 & Instruction 613 \\
\hline $7-9$ & 611 & 612 & 613 \\
\hline $10-18$ & $\Delta \mathrm{X} 0$ & $\Delta \mathrm{X} 0$ & - \\
\hline $19-27$ & $\Delta \mathrm{YO}$ & $\Delta \mathrm{YO}$ & 一 \\
\hline $28-36$ & $\triangle \mathrm{SCAL1}$ & 一 & $\triangle$ SCAL1 \\
\hline $37-45$ & $\begin{array}{l}\text { SCAL } 2 \text { or } \\
\text { probability }\end{array}$ & $\begin{array}{l}\text { SCAL } 2 \text { or } \\
\text { probability }\end{array}$ & $\begin{array}{l}\text { SCAL } 2 \text { or } \\
\text { probability }\end{array}$ \\
\hline
\end{tabular}

\subsubsection{Atom Plotting Instructions (700 Series)}

These instructions produce various representations of the atom based on the familiar balland-stick molecular model. In the general case, the ball is an ellipsoid representing a contour surface of equal probability density of thermal motion displacement. Alternatively, when thermal 
motion is not being portrayed, the ball can be a sphere of arbitrary dimension. The 700 series also has provision for labeling the atomic site with the corresponding chemical symbol. The instructions in this series draw the atoms in the ATOMS array that project onto the usable part of the drawing area, defined with the 301 instruction. Atoms found to be out of bounds are bypassed, and a Fault Message (NG = 10) (see 3.4) is printed in the ORTEP output file. An atom is out of bounds under the following conditions: (1) its $z$ coordinate in the scaled reference Cartesian system is greater than $1 / 2$ the viewing distance, (2) its center after projection falls outside the limiting boundary of the drawing board, or (3) its projected center falls in the outermost $3 / 4$ of the drawing margin. (See 3.3.4.1.)

An ellipsoid, for graphical purposes in ORTEP, is considered to be composed of ellipses and straight lines. The ellipses are of two types-principal ellipses and boundary ellipses. Relative to the viewpoint, a principal ellipse is further subdivided into a front half and a back, or hidden, half. There are three principal ellipses per ellipsoid, corresponding to the three principal planes. The boundary ellipse is the edge of the ellipsoid as seen from the viewpoint. The front and back halves of the principal ellipses meet at the boundary ellipse. The straight line segments of the ORTEP ellipsoid are the forward principal axes, reverse principal axes, and octant-shading lines.

Fig. 3.1 shows various combinations of these elements along with the ORTEP instruction number and parameter values to produce each. It is obvious that certain of these combinations are better representations than others. Instructions 701, 702, 703, and 706 generate specific ellipsoid types as shown in Fig. 3.1. Instruction 704 draws the boundary ellipse alone. If an atom is entered as a sphere, the boundary will be circular before projection and slightly elliptical after perspective projection. Instruction 705 allows the user to make up any representation from the basic components.

Chemical symbols up to six alphanumeric characters in length may be included with each atom in the ORTEP input file. These symbols can be put onto the illustration with one 700 series or several 900 series instructions. The 700 series places the center of the six-character field of each atom in the same position relative to the atom center for all the drawn atoms; the 900 series allows the user to position each symbol individually. The 700 series requires only three parameters as follows: (1) symbol height in inches, (2) parallel (left/right) offset in inches, and (3) perpendicular (up/down) offset in inches. The parameters refer to the model before projection, and they will change slightly during perspective projection. The parallel and perpendicular offset refer to the exact center of the six-character input field and are relative to the lettering base line set up with the 302 instruction. A symbol height of " 0 " or blank will cause the symbol drawing routine to be bypassed. If NPLANE $=0$ in a 705/715 instruction and the symbol height is greater than zero, then chemical symbols alone are drawn on the atomic sites. 


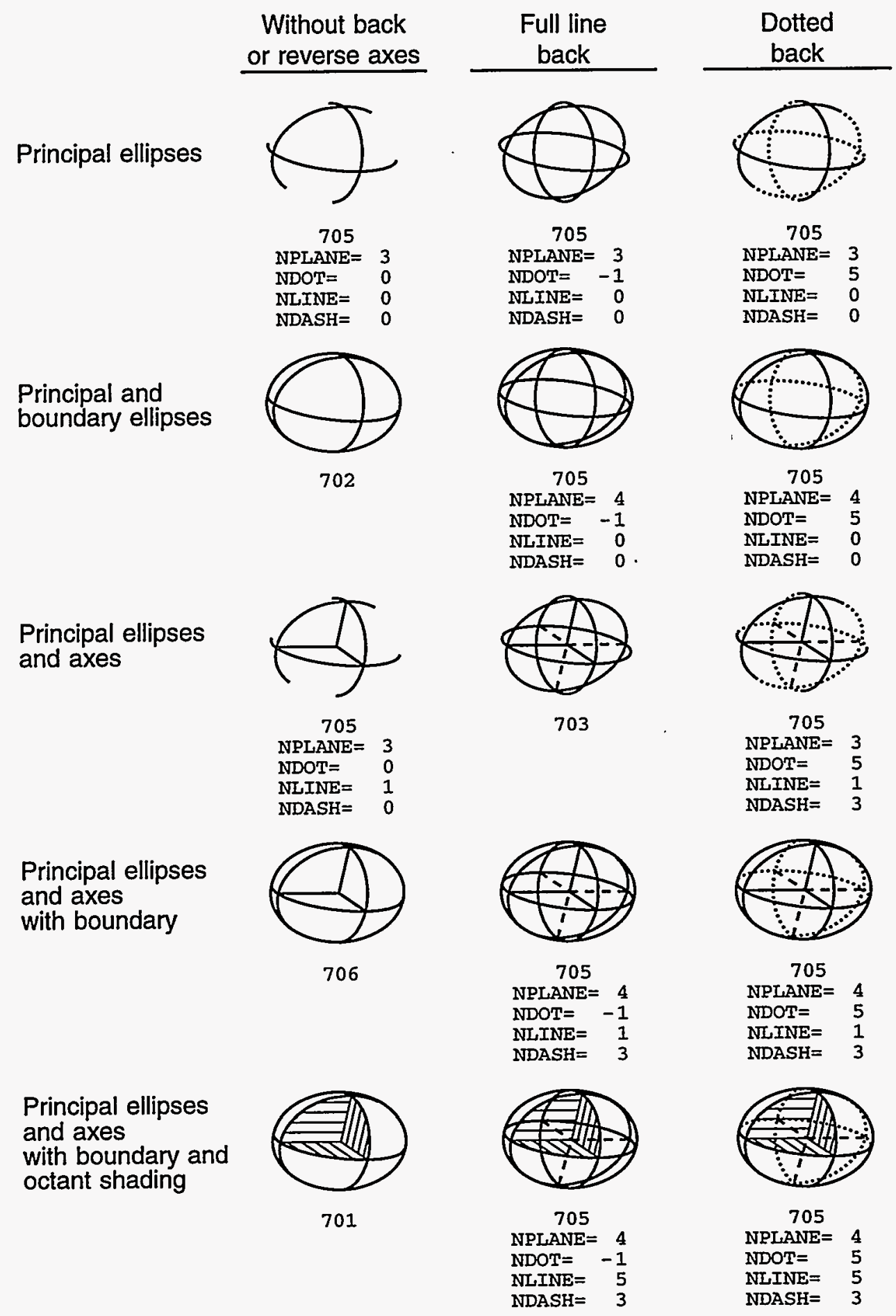

Fig. 3.1. Various combinations of ellipsoid components showing ORTEP instruction number and parameter values to produce each. 
It is possible to vary the thickness of the boundary ellipse line by making it a function of $z$, the height of the atom from the drawing board. This option is normally used with the 704 (boundary only) instruction but will work for any 700 instruction. Entries are put in the $A_{0}$ and $A_{1}$ fields of the instruction continuation card to specify the coefficients of

$$
\Delta \mathrm{R}(z)=\mathrm{A}_{0}+\mathrm{A}_{1} z
$$

where

$\Delta \mathrm{R}$ is the increase in radial dimension to be added to the width of the single pen line, $\mathrm{A}_{0}$ is $\Delta R$ for an atom at $z=0$, and

$A_{1}$ is the rate of increase in radial dimension with $z$.

Example: Assume the atoms of a scaled model range from 5 in. below to 5 in. above the drawing board and the pen width is $0.2 \mathrm{~mm}$ (.008 in.). If we want the closest ellipse boundary to be five times as thick as the farthest, then $\Delta \mathrm{R}(-5 \mathrm{in})=$. $0, \Delta R(5)=0.008 \times(5-1)=0.032$ in.; thus $A_{0}=0.016$ in. and $A_{1}=0.0032$ in.

The program widens the line by stepping radially in increments of DISP, which is set by primer constant to 0 . in. A 303 instruction must be used to give DISP a positive value for this line thickening process to work.

Selected types of atoms from the ATOMS array can be drawn without having to alter the contents of the array. This is accomplished by using a number run (NR) code that includes the atom types that are to be drawn with a particular 700-series instruction. This feature is particularly useful when two or more different atom representations are used, such as for the carbon and hydrogen atoms in the cubane example. If no NR is entered, then all atoms in the array are drawn.

The ORTEP file output for the 701-706 instructions consists of the following:

- $x, y$ plotter coordinates: the coordinates, in inches, for the projected atom center on the plot, measured from the lower left-hand corner of the limiting boundary. This is the fixed plotter coordinate system with the origin set by the plotter driver.

- $x, y, z$ working Cartesian coordinates: the coordinates, in inches, for the oriented and scaled atomic model before projection. The $x$ and $y$ axes parallel the plotter $x$ and $y$ axes, and the origin of the system is in the plane of the plotter at the point $x_{0}, y_{0}$ (see 3.3.7) in plotter coordinates. The point ORGN of the scaled model is at this point (see 3.3.6.1).

- $x, y, z$ triclinic coordinates, in fractions of the unit-cell edges relative to the crystal unit-cell origin.

- Principal axes of thermal motion, consisting of (a) principal values of rootmean-square displacement and (b) direction cosine for principal vectors relative to the working Cartesian system.

- The atom designator code and chemical symbol for the atom.

Instructions 711-716 are identical to 701-706 except that the $71 n$ series suppresses all ORTEP file output except fault messages. 


\begin{tabular}{|c|c|c|}
\hline Columns & $\begin{array}{c}\text { Instructions } \\
\mathbf{7 0 1}, \mathbf{7 0 2}, \mathbf{7 0 3}, \mathbf{7 0 4}, \mathbf{7 0 6}, \\
\mathbf{7 1 1}, \mathbf{7 1 2}, \mathbf{7 1 3}, \mathbf{7 1 4}, \mathbf{7 1 6}\end{array}$ & $\begin{array}{l}\text { Instructions } \\
705 \text { and } 715\end{array}$ \\
\hline 3 & $\begin{array}{l}1 \text { (if atom selection or } \\
\text { boundary retracing is } \\
\text { desired; otherwise } 0 \text { ) }\end{array}$ & $\begin{array}{l}1 \text { (if atom selection or boundary } \\
\text { retracing is desired; otherwise 0) }\end{array}$ \\
\hline $7-9$ & $\begin{array}{l}701,702,703,704,706 \\
711,712,713,714, \text { or } 716\end{array}$ & 705 or 715 \\
\hline $10-18$ & - & $\begin{array}{l}\text { NPLANE } \\
\quad=0: \text { no ellipsoid components } \\
\quad=1: \text { boundary ellipse only } \\
=3: \text { principal ellipses only } \\
=4: \text { boundary + principal ellipses }\end{array}$ \\
\hline $19-27$ & - & $\begin{array}{l}\text { NDOT (back side of principal ellipses) } \\
\text { <0: } \quad \text { solid line back side } \\
\text { =0: } \quad \text { back side omitted } \\
=3: \quad 4 \text { dots on back side } \\
=4: \quad 8 \text { dots on back side } \\
=5: \quad 16 \text { dots on back side } \\
=6: \quad 32 \text { dots on back side }\end{array}$ \\
\hline $28-36$ & - & $\begin{aligned} \text { NLINE (forward principal axes and shading) } \\
\quad=0: \quad \text { no forward axes or shading } \\
\quad=1: \quad \text { forward principal axes only } \\
=N: \text { forward axes }+(N-1) \text { line shading }\end{aligned}$ \\
\hline $37-45$ & - & $\begin{array}{l}\text { NDASH (dashed reverse principal axes) } \\
\quad=0: \quad \text { no reverse axes } \\
\quad=N: \text { dashed reverse axes with } N \text { dashes }\end{array}$ \\
\hline $46-54$ & Symbol height (in.) & Symbol height (in.) \\
\hline $55-63$ & Parallel offset (in.) & Parallel offset for symbols (in.) \\
\hline \multirow[t]{8}{*}{6472} & Perpendicular offset (in.) & Perpendicular offset for symbols (in.) \\
\hline & \multicolumn{2}{|c|}{$\begin{array}{c}\text { Format No. } 1 \\
\text { Parameter Continuation }\end{array}$} \\
\hline & Columns & (if used) \\
\hline & $10-18$ & {$\left[\mathrm{~A}_{0}\right.$ (in.) $]$} \\
\hline & $19-27$ & {$\left[\mathrm{~A}_{1}\right.$ (in.) $]$} \\
\hline & $28-36$ & [NR (from) \\
\hline & $37-45$ & $\mathrm{NR}(\mathrm{to})]$ \\
\hline & 54 & [NR type] \\
\hline
\end{tabular}




\subsubsection{Bond Plotting Instructions (800 Series)}

The bond plotting instructions are grouped into two general types, explicit and implicit, depending on how the bonds are specified. Explicit bonds require a vector designator code (see 3.1.2) for each bond. Implicit bonds make use of vector search codes (see 3.1.5) to find pairs of atoms from the ATOMS array set up by the 400 series instructions.

There are two types of bonds that can be drawn, line bonds and stick bonds. The line bond is a very crude, but rapid, method useful in drawing preliminary illustrations. It is constructed by placing the symbol $*$ on the two atom sites and drawing a single straight line between them. Line bonds are always specified implicitly.

The more elaborate bond is the stick bond, which could also be called a conical bond because of its accentuated perspective taper. (The accentuated taper may be increased or decreased by changing the value assigned to TAPER in subroutine PRIME.) Each end of the bond intersects either an ellipsoid or an enveloping cone (tangent cone) that has its apex at the viewpoint and is tangent to an ellipsoid. In general, the ellipsoid intersection.is automatically used if the axis of the bond intersects the ellipsoid at a point that is visible to the viewpoint; otherwise, the tangent cone intersection is used, so that the bond appears to terminate at the boundary of the ellipsoid. However, the user can specify that the ellipsoid intersection always be used in order to make the skeleton type model (such as produced by the 703 instruction) appear even more transparent. The radius of the stick bond and the number of lines used to draw the bond are specified by input parameters.

Bond-distance labels can be drawn automatically with stick bonds but not with line bonds. The bond-distance label numbers are in Angstrom units with one, two, or three places past the decimal point. The bond labels on the illustration will have their base lines parallel to the stick bonds and will be right side up for the viewer. The height of the label in inches and the perpendicular offset distance for the center of the label relative to the center of the bond are parameters to be specified by the user. With the current primer constant for FORE, if the sine of the angle between the bond and the mean viewing vector is greater than 0.5 , the lettering is done in perspective along the bond. When the sine of the angle is less than 0.5 , the perspective lettering would be excessively foreshortened; the lettering is then made parallel to the plane of the drawing with its base line parallel to the projected bond. Different lettering heights and different perpendicular offset distances can be assigned to the perspective and nonperspective bond-distance labels.

All 800 instructions require Format No. 2 trailer cards to provide a number of bond parameters as follows:

- Bond type (for stick bonds) is designated by an integer NBOND, where $-5 \leq$ $\mathrm{NBOND} \leq 5$. The negative integers denote that both ends of the bonds terminate at the ellipsoids. The positive integers denote bonds ending either at the ellipsoid or the tangent cone, as described previously in this section. An entry of zero draws no bond. A magnitude of 1 for NBOND produces two lines, one for each bond edge, $180^{\circ}$ apart in the plane normal to the bond axis. Lines are drawn $90^{\circ}, 45^{\circ}, 22.5^{\circ}$, or $11.25^{\circ}$ apart for NBOND magnitudes of $2,3,4$, or 5 , respectively. The back side of the bond is not drawn. 
- The bond radius (mean value for stick bonds) is in Angstrom units. Values between 0.01 and $0.06 \AA$ usually give good results. Any positive value may be used as long as it is smaller than the scaled ellipsoid minimum semidimension. The bond radius is not changed by the ellipsoid scale factor ratio SCAL2. The bond radius should not be made "vanishingly small" (e.g., $r<$ $0.005 \AA$ ) if the overlap feature (see 3.3.12) is used because numerical rounding may cause incorrect hidden-line elimination.

- The height of perspective labels for bond distances is entered as zero if no bond distances are to be labeled. Positive values denote the lettering height in inches before projection.

- The perpendicular offset for bond distance perspective labels (in inches) specifies the offset of the center of the distance label relative to the center of the bond.

- The height of regular labels for bond distances is entered as zero if no bond distances corresponding to foreshortened bonds are to be drawn. Positive values give the lettering height in inches before projection.

- The perpendicular offset for bond-distance regular labels (in inches) specifies the offset of the center of the distance label relative to the center of the bond.

- The significant digits indicator is $-1,0$, or 1 , denoting bond distance labels with one, two, or three digits, respectively, after the decimal place.

Instructions 801,802 , and 803 differ from 811,812 , and 813 only in the ORTEP output file listing. The second group has no output except error messages. The first group lists: plotter coordinates in inches, scaled Cartesian coordinates (in inches) of atoms before projection, and triclinic crystal coordinates for the atoms of each bond. The interatomic bond distance in Angstroms is also listed. If an atom of a bonded pair is out of bounds, a fault message (NG =10) is printed in the ORTEP output file. If the bond is hidden and cannot be drawn, fault message NG = 14 is printed. Fault NG = 13 signifies that an imaginary intersection was found with a bond radius larger than the ellipsoid semidimension. (See 3.4.)

\subsubsection{Explicit Bonds}

Explicit stick bonds are produced with the $801 / 811$ instructions. The bonds are described with two atom designator codes for each bond. The atom designator codes go on the 801/811 card and on Format No. 1 trailer cards if more than three atom pairs are needed. The two atom designator codes for a bond must be in adjacent fields, but blank fields can be inserted between the different bonds. Since there are seven fields available per card, it is a good idea to use only two, four, or six of them so that the card sequence within the instruction (other than first and last cards) will be unimportant. As mentioned earlier, a Format No. 2 trailer card is required. 
Instructions

\begin{tabular}{c} 
Columns \\
\hline 3 \\
$7-9$ \\
$10-18$ \\
$19-27$ \\
$28-36$ \\
$37-45$ \\
$46-54$ \\
$55-63$
\end{tabular}

\section{1/811}

2 (or 1 if more

than 3 pairs of

ADCs are needed)

801 or 811

$\mathrm{ADC}_{1}$ (from)

$$
\mathrm{ADC}_{1} \text { (to) }
$$

$\left[\mathrm{ADC}_{2}\right.$ (from)

$\mathrm{ADC}_{2}$ (to)]

[...

...]
Columns

3

22-24

$37-42$

43-48

49-54

55-60

61-66

67-72
Format No. 2

Continuation Card

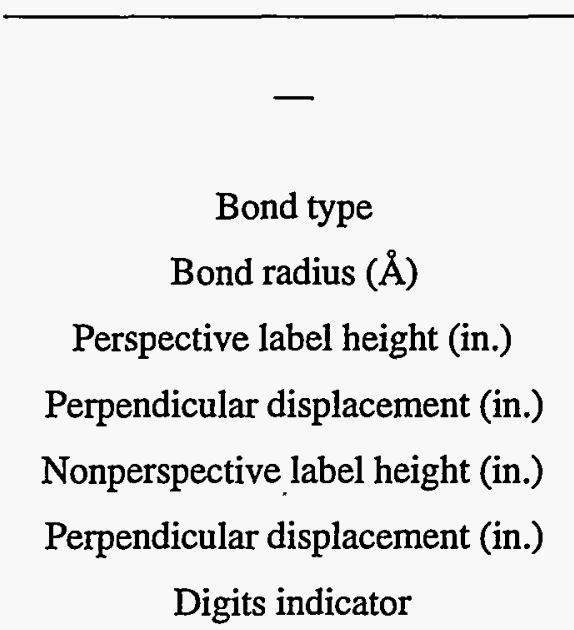

\subsubsection{Implicit Bonds}

Instructions 802/812 are used for implicit stick bonds, and 803/813 are used for implicit line bonds. The symbol drawn on the atomic sites by the $803 / 813$ instructions may be made larger or smaller by redefining the SCAL2 factor, which is controlled by the 600 series of instructions (see 3.3.7). Number run type takes a non-zero (or non-blank) value only when working with atom "features" (see 3.1.10-12 and 4.6). At least one Format No. 2 trailer card is required.

The use of vector search code (VSC) cards for the implicit bond plotting instructions has been extended to include a provision for drawing coordination polyhedra while suppressing the unwanted bonds. In addition to describing the bond with origin and target number runs and the $D_{\min }$ to $D_{\max }$ range, a condition can be imposed to require that both atoms must be within a specified "polyhedral distance range" of an atom in the ATOMS array that is included in a "polyhedron" number run. This option is brought into play by a negative value in columns 43 to 48 of the VSC card.

\begin{tabular}{ccc} 
Columns & & $\begin{array}{c}\text { Instructions } \\
\mathbf{8 0 2 / 8 1 2} \text { and } \mathbf{8 0 3 / 8 1 3}\end{array}$ \\
\cline { 1 - 1 } 3 & & 2 \\
$7-9$ & & 802 or 812 \\
$10-18$ & & - \\
27 & & NR type
\end{tabular}


Instructions 802/812

Format No. 2 Vector Search Code

\begin{tabular}{|c|c|c|c|c|}
\hline \multirow[b]{2}{*}{ Columns } & \multicolumn{2}{|c|}{ Formal so. 2 rector sear con coue } & \multicolumn{2}{|c|}{ 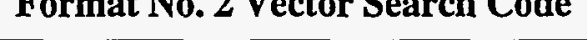 } \\
\hline & $\begin{array}{c}\text { Positive value } \\
\text { in col. } 43-48\end{array}$ & $\begin{array}{c}\text { Negative value } \\
\text { in col. } 43-48\end{array}$ & $\begin{array}{l}\text { Positive value } \\
\text { in col. } 43-48\end{array}$ & $\begin{array}{c}\text { Negative value } \\
\text { in col. } 43-48\end{array}$ \\
\hline 3 & 0 or 2 & 0 or 2 & 0 or 2 & 0 or 2 \\
\hline $10-12$ & Org. NR (from) & Org. NR (from) & Org. NR (from) & Org. NR (from) \\
\hline $13-15$ & Org. NR (to) & Org. NR (to) & Org. NR (to) & Org. NR (to) \\
\hline $16-18$ & Tar. NR (from) & Tar. NR (from) & Tar. NR (from) & Tar. NR (from) \\
\hline $19-21$ & Tar. NR (to) & Tar. NR (to) & Tar. NR (to) & Tar. NR (to) \\
\hline $22-24$ & Bond type & Bond type & - & 一 \\
\hline $25-30$ & $D_{\min }(\AA)$ & $D_{\min }(\AA)$ & $D_{\min }(\AA)$ & $D_{\min }(\AA)$ \\
\hline $31-36$ & $D_{\max }(\AA)$ & $D_{\max }(\AA)$ & $D_{\max }(\AA)$ & $D_{\max }(\AA)$ \\
\hline $37-42$ & Bond radius $(\AA)$ & Bond radius $(\AA)$ & - & - \\
\hline $43-48$ & $\begin{array}{l}\text { Perspective } \\
\text { label hgt. (in.) }\end{array}$ & $\begin{array}{l}\text { Polyhedron } \\
\text { NR (from) }\end{array}$ & - & $\begin{array}{l}\text { Polyhedron } \\
\text { NR (from) }\end{array}$ \\
\hline $49-54$ & $\begin{array}{c}\text { Perpendicular } \\
\text { displacement (in.) }\end{array}$ & $\begin{array}{l}\text { Polyhedron } \\
\text { NR (to) }\end{array}$ & - & $\begin{array}{l}\text { Polyhedron } \\
\text { NR (to) }\end{array}$ \\
\hline $55-60$ & $\begin{array}{l}\text { Nonperspective } \\
\text { label hgt. (in.) }\end{array}$ & $\begin{array}{l}\text { Polyhedron } \\
D_{\min }(\AA)\end{array}$ & - & $\begin{array}{l}\text { Polyhedron } \\
D_{\min }(\AA)\end{array}$ \\
\hline $61-66$ & $\begin{array}{c}\text { Perpendicular } \\
\text { displacement (in.) }\end{array}$ & $\begin{array}{l}\text { Polyhedron } \\
D_{\max }(\AA)\end{array}$ & - & $\begin{array}{l}\text { Polyhedron } \\
D_{\max }(\AA)\end{array}$ \\
\hline $67-72$ & Digits indicator & - & & \\
\hline
\end{tabular}

Instructions 803/813

Format No. 2 Vector Search Code 
ORTEP-III provides the following character set for labels:

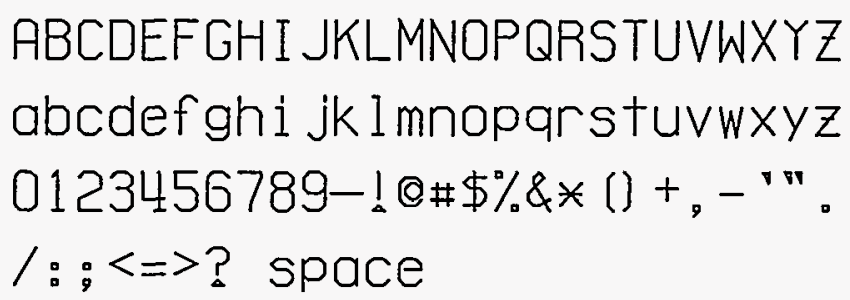

General titles and bond length labels can be drawn either in perspective or parallel to the plane of the drawing (nonperspective). Chemical symbols are always drawn parallel to the plane. Instructions 913-916 produce perspective lettering, and instructions 901-906 produce regular plane lettering.

Two vectors, the upright vector and the base-line vector, are needed to describe a lettering plane. In ORTEP, the upright lettering vector is always parallel to the plane of the drawing. For perspective lettering the base-line vector is a general vector in three dimensions. In the nonperspective case, the base-line vector is either along the projection of a general vector or along the vector (in the plane of the drawing) that is oriented with a 302 title rotation instruction (theta base line). If theta is zero, then the theta base-line vector is along the plotter positive $x$ axis.

The exact center of the label is always referred to when specifying the position of the label. The program goes through the following steps to position the center point of the label onto the drawing.

(1) A point $P 1$ is found that is either the position of atom A (columns 10-18) or the mean of two atom positions (atom $A$ and atom $B$ ) if an atom designator exists in the atom B field (columns 19-27) of the instruction card.

(2) A point $P 2$ is found by (a) translating from $P 1$ along the base-line vector for the distance specified by the parallel offset and then (b) translating along the upright vector by the perpendicular offset distance.

(3) A point $P 3$ is found by projecting $P 2$ onto the plane of the drawing.

(4) If the $x$ edge reset is greater than 0 , then $x$ is reset to this value. If $x$ edge reset is less than $0, x$ is reset to the positive $x$ plot boundary minus $1 x$ edge reset . No resetting is done if $x$ edge reset equals 0 . The $y$ parameter is handled in the same manner with $y$ edge reset.

Instruction 901 produces a nonperspective atom label with theta base line, using the atom label for atom $\mathrm{A}$.

A nonperspective title with theta base line is drawn with the 902 instruction. The title must be provided with the instruction on a Format No. 3 trailer card. The title should be centered about columns 36-37 of that card. However, if the title begins in column 1, it will be centered automatically (a new feature in ORTEP-III). 
A nonperspective general title with normal plane lettering is produced by the 903 instruction and with perspective lettering by 913 . The general vector is from atom $A$ to atom $B$. The title must be provided with the instruction on a Format No. 3 trailer card. The title should be centered about columns $36-37$ of that card. If the title begins in column 1 , it will be centered automatically.

Instructions $904,905,906,914,915$, and 916 produce bond-length labels. The first three are for nonperspective lettering with one, two, and three places after the decimal point, respectively; and the last three are for the corresponding bond-length labels with perspective. The general vector is from atom $A$ to atom $B$. Note that the sense of the vector is important in order to have the label right side up.

The format for the entire 900 series is as follows:

\begin{tabular}{|c|c|}
\hline Columns & 900 Instructions \\
\hline 3 & 0 (or 3 for 902,903 , and 913 ) \\
\hline $7-9$ & $9 n n$ (instruction number) \\
\hline $10-18$ & $\mathrm{ADC}$ for atom $\mathrm{A}$ \\
\hline $19-27$ & {$[\mathrm{ADC}$ for atom $\mathrm{B}]$} \\
\hline $28-36$ & $x$ edge reset (in.) \\
\hline $37-45$ & $y$ edge reset (in.) \\
\hline $46-54$ & Lettering height (in.) \\
\hline $55-63$ & Parallel offset (in.) \\
\hline $64-72$ & Perpendicular offset (in.) \\
\hline
\end{tabular}

\subsubsection{Save Sequence Instructions (1100 Series)}

It is often desirable to repeat a sequence of instructions one or more times with other instructions inserted between the repetitions. The 1100 series allows the user to do this without the necessity of putting in duplicate sequences of instruction cards. It is not an elaborate looping device, but it does give additional flexibility to the system.

The three instructions in this series are 1101 to start the save sequence, 1102 to terminate the save sequence, and 1103 to execute the save sequence. All instruction cards and their trailer cards between the 1101 and 1102 instructions are executed and saved. Each subsequent 1103 instruction then repeats all the saved instructions. There are no parameters to be entered with the 1100 series instructions.

Instructions

$\frac{\text { Columns }}{6-9} \quad \frac{1101,1102 \text {, and } 1103}{1101,1102, \text { or } 1103}$




\subsubsection{Overlap Correction Instructions (1001, 821, 822)}

(Instruction 511 in OR TEP-II ${ }^{2}$ has been renumbered to 1001 in ORTEP-III. Instruction 511 continues to work as it did in OR TEP- $\Pi,{ }^{2}$ but users should use 1001 in the future.)

Instruction 1001 activates the hidden line removal feature of ORTEP, which corrects the illustration for overlapping atoms and bonds. It stores the projected atom boundary ellipses for all atoms in the ATOMS array. It is important that the contents of the ATOMS array, the scaling and positioning, and the structure orienting parameters (controlled by the 400,600 , and 500 series of instructions, respectively) not be changed between the 1001 instruction and the drawing of the atoms and bonds by the 700 and 800 series of instructions. Otherwise, the projected outlines may be destroyed or become inappropriate.

The projected outlines of bonds are approximated as quadrangles. Those of implicitly specified bonds may be stored for overlap correction by adding Format No. 2 trailer cards to the 1001 instruction that are identical to those of the 802/812 instructions used subsequently for plotting the implicit bonds. Alternatively, implicitly specified bonds may be stored with one or more 822 instructions with the Format No. 2 trailer card set. A 1001 instruction must precede the first 822 . If more than one $802 / 812$ instruction is used to draw the bonds, there probably should be a corresponding 822 provided for each.

Explicit bonds may only be stored by using one or more 821 instructions following a 1001 instruction. These will be identical to the $801 / 811$ instructions used subsequently for plotting the explicit bonds except for the instruction number.

As currently dimensioned, the maximum numbers of projected atoms and projected bonds that can be stored are 500 and 599, respectively. A list of the projected atoms and bonds is given in the ORTEP output file.

The projected outline information for atoms and bonds must be recalculated for each member of a stereo pair; consequently the 1001 and 821 or 822 instructions are usually the first instructions within the save sequence. The old overlap information is deleted whenever a new 500 or 600 series or 1001 instruction is executed.

All details inside atoms and bonds, including chemical symbols drawn with 700 series instructions and bond-distance labels drawn with 800 series instructions, will be corrected for overlap. Lettering drawn by the 700 and 800 series instructions that is outside the atom or bond boundaries will not be corrected for overlap unless the overlying atom or bond outline actually intersects (in projection) the atom or bond being drawn. Labels and symbols drawn with the 900 series instructions will not be corrected for overlap.

\subsubsection{Instruction 1001, Projected Outline Storage}

A constant width overlap margin (i.e., a blank strip at the intersection of overlapping elements) is included in the dimensions of each projected atom ellipse and projected bond quadrangle. The width of this margin (in inches) may be specified as a parameter with the 1001 instruction if desired; otherwise, the margin is set by default. The default value is calculated as follows: 


$$
\begin{aligned}
& \text { for } \mathrm{SCAL1}<0.25: \quad \text { OVMRGN }=\max \left(0.010, \mathrm{SCALl}^{1 / 2} \times 0.05\right) \\
& \text { for } \operatorname{SCAL1} \geq 0.25: \quad \text { OVMRGN }=\max \left(0.025, \mathrm{SCALl}^{1 / 2} \times 0.03\right)
\end{aligned}
$$

\begin{tabular}{|c|c|}
\hline Columns & Instruction 1001 \\
\hline 3 & 2 (if bonds are to be stored; otherwise 0 ) \\
\hline $6-9$ & 1001 \\
\hline $10-18$ & $\begin{array}{l}\text { Overlap Margin } \\
0: \text { default value (described above) } \\
1: 0.0 \text { in. } \\
\text { Value in range } 0-1.0 \text { in. }\end{array}$ \\
\hline 27 & NR type \\
\hline
\end{tabular}

Some users prefer an overlap margin of 0.0 for stereoscopic drawings.

If the value in column 3 is "2", Format No. 2 trailer cards are included. In general, all the trailer cards included with the $802 / 812$ instructions are used. (See 3.3.9 and 3.3.12.3.)

New on this card in ORTEP-III is the second parameter, which specifies the number run type of the number runs that follow on the Format No. 2 trailer cards.

\subsubsection{Instruction 821, Explicit Bond Outline Storage}

If explicit bonds are to be stored for the overlap correction, the attached atoms must be in the ATOMS array even though this is not a requirement for the $801 / 811$ instructions used for plotting the explicit bonds. In general, the 821 instructions will be identical to the $801 / 811$ instructions (except for the instruction number) used subsequently for plotting the explicit bonds. (See 3.3.9.)

\subsubsection{Instruction 822, Implicit Bond Outline Storage}

Usually, the information on implicit bond outlines is stored with the 1001 card. However, if more than 20 Format No. 2 trailer cards are needed to specify all bonds, the extra ones can be entered with this instruction. Also, 822 instructions in addition to the 1001 may be used to treat different atom features in different ways. In general, the 822 instructions will be identical to the $802 / 812$ instructions (except for the instruction number) used subsequently for plotting the implicit bonds. (See 3.3.9.)

$\begin{array}{ccc}\text { Columns } & & \text { Instruction } \mathbf{8 2 2} \\ 3 & 2 \\ 7-9 & 822 \\ 10-18 & - \\ 27 & \text { NR type }\end{array}$




\subsubsection{Termination Instructions (Negative Series)}

The -1 instruction terminates ORTEP, exiting the program via subroutine EXITNG.

The -2 instruction terminates the current structure and reinitializes ORTEP to read another structure from the same input file starting with a new title card. As many structures as desired may be cascaded in the input file in this manner before exiting with a -1 instruction. The user's initial input about the destinations for the drawing and text output hold for the entire set of structures. At the end of each structure, the user is offered the opportunity to save the drawing, view it on the screen, or edit the current instruction set before proceeding to the next structure.

(See 4.1.).

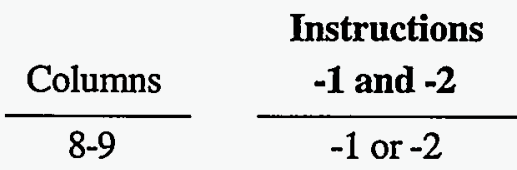

\subsubsection{Supplementary Instructions}

The instructions available in ORTEP-III have numbers less than 1200. Any instruction in the input file with a number greater than or equal to 1200 will cause ORTEP to branch to subroutine SPARE to execute the code found there. As distributed, this is a dummy subroutine containing the single FORTRAN statement RETURN. It is provided to give users a simple way to add their own instructions without having to understand the full program logic. Subroutine SPARE has a single parameter INST, which is the instruction number.

\subsection{LIST OF FAULT INDICATORS}

ORTEP checks for certain errors, and when one of these occurs, an error message, "FAULT NG $=N G A D C m$ " is written in the ORTEP output file. The meanings of fault indicator number $N G$ is explained in Table 3.1. $A D C$ and $m$ identify the atom code and the instruction involved (if these are relevant). If possible, corrective measures are made by ORTEP and the calculation proceeds; otherwise, the job is terminated by calling subroutine EXITNG. 
Table 3.1. ORTEP fault indicators.

\begin{tabular}{|c|c|c|c|}
\hline NG & $\begin{array}{l}\text { Subroutine } \\
\text { Involved }\end{array}$ & Fault & Action \\
\hline 1 & PRELIM & $\begin{array}{l}\text { No sentinel found after read- } \\
\text { ing } 96 \text { symmetry cards }\end{array}$ & Tries to read parameter cards \\
\hline 2 & PRELIM & $\begin{array}{l}\text { No sentinel found after read- } \\
\text { ing the parameter cards for } \\
505 \text { atoms }\end{array}$ & Tries to read instruction cards \\
\hline 3 & PRELIM & $\begin{array}{l}\text { Anisotropic temperature factor } \\
\text { coefficients form a matrix } \\
\text { which is not positive definite }\end{array}$ & $\begin{array}{l}\text { EXIT after printing out all } \mathrm{rms} \\
\text { principal values (imaginary } \\
\text { ones are listed negative) }\end{array}$ \\
\hline 4 & ATOM, PAXES & $\begin{array}{l}\text { Symmetry operation number is } \\
\text { higher than the number of } \\
\text { input operations }\end{array}$ & Omit atom \\
\hline 5 & ATOM, PAXES & $\begin{array}{l}\text { Atom number is higher than } \\
\text { the number of input atoms }\end{array}$ & Omit atom \\
\hline 6 & EIGEN & $\begin{array}{l}\text { Null temperature factor matrix } \\
\text { or failure in bisection routine }\end{array}$ & $\begin{array}{l}\text { EXIT, after printing out all } \\
\text { principal values }\end{array}$ \\
\hline 7 & EIGEN & $\begin{array}{l}\text { Eigenvector routine failure } \\
\text { due to null vector }\end{array}$ & $\begin{array}{l}\text { EXIT, after printing out all } \\
\text { principal values }\end{array}$ \\
\hline 8 & INITSC & Error initializing screen driver & EXIT \\
\hline 9 & MAIN, SPARE & $\begin{array}{l}\text { Unidentified instruction num- } \\
\text { ber }\end{array}$ & Omit faulty instruction \\
\hline 10 & BOND, F700 & Atom out of bounds & Omit atom \\
\hline 11 & F800 & No vector search codes & Omit instruction \\
\hline 12 & F600, SEARC & $\begin{array}{l}\text { Insufficient number of atoms } \\
\text { in ATOMS array }\end{array}$ & EXIT \\
\hline 13 & BOND & $\begin{array}{l}\text { Imaginary bond intersection } \\
\text { (i.e., bond larger than atom) }\end{array}$ & Omit bond \\
\hline 14 & BOND & Hidden (end-on) bond & Omit bond \\
\hline 15 & F900 & Null vector as base line & Omit label \\
\hline 16 & STORE & ATOMS array is full & Omit all succeeding atoms \\
\hline 17 & LAP700, LAPAB & $\begin{array}{l}\text { Maximum number of } \\
\text { projected ellipses ( } 20) \text { over an } \\
\text { atom or bond to be drawn } \\
\text { exceeded }\end{array}$ & $\begin{array}{l}\text { Additional ellipses not } \\
\text { corrected for overlap }\end{array}$ \\
\hline 18 & LAP800, LAPAB & $\begin{array}{l}\text { Maximum number of quadran- } \\
\text { gles }(30) \text { over an atom or bond } \\
\text { to be drawn exceeded }\end{array}$ & $\begin{array}{l}\text { Additional quadrangles } \\
\text { (bonds) not corrected for over- } \\
\text { lap }\end{array}$ \\
\hline
\end{tabular}




\section{USING ORTEP-III}

This section provides some general information on using ORTEP-III, and it describes how some aspects of the program work to help users who wish to modify the program's operation.

\subsection{USER INTERFACE}

ORTEP-III is a semi-interactive program that requests certain control information from the user while the program executes. A default value for each item is provided in square brackets, and it will be used if the user simply hits the RETURN key (on some keyboards, the name of this key is ENTER). When ORTEP starts, the user is asked to supply three basic items of information.

Enter instruction set file name or "exit" [TEP.IN]:

Drawing to (1) Screen, (2) Postscript file, (3) HPGL file, or (0) Omit [1]:

Text output to (1) File, (2) Screen, or (0) Omit [0] :

The first of these is the ORTEP input file name with a default name of TEP.IN. The user may supply a file name or may enter "exit" or "EXIT" to stop program execution. Care must be taken when entering a file name to match the case of the letters on those systems that distinguish upper and lower case.

The second item requested is the destination of the ORTEP drawing with the default being the screen. The alternatives of an Encapsulated Postscript (EPS) file or HPGL/2 (HewlettPackard Graphics Language) file are chosen if the user enters a " 2 " or " 3 ", respectively. A choice of " 2 " or " 3 " causes ORTEP to ask about the orientation of the drawing.

(1) Portrait or (2) Landscape orientation [1]:

One further piece of information is needed for an EPS landscape drawing.

How tall is printer page in inches? [11.00]:

If the ORTEP illustration is saved in a file, the file will be named TEPnnn.PRN where $n n n$ is a number starting with the value 001 . The program sets the value so old illustration files are not overwritten. Once the file name is determined by the program, the name is displayed on the screen. If a different naming convention is preferred, the code for naming the files is in subroutines INITPS and INITHP.

The third main user inquiry is the destination of ORTEP's text output. The default value here is to omit the output. An entry of " 2 " displays the output on the screen. If a " 1 " is entered to save the output in a file, the user is asked to supply a file name.

Enter output file name [TEP.out]:

The default name is derived from the name of the input file by adding or substituting the extension ".out". A different output file name may be typed in. CAUTION: If the name chosen here is the same as an existing file, it will be overwritten. 
Before the program exits, the user is given another opportunity to save the drawing or view it on the screen. In addition, an option to use ORTEP's internal editor is provided.

(1) Save drawing as Postscript file

(2) Save drawing as HPGL file

(3) Redraw structure on screen

(4) Edit instruction set

[Quit] :

The wording of the user requests and the code to handle the user input are in subroutine UINPUT. Default values are set in subroutine DFLTS and held in COMMON DFL.

\title{
4.2 SCREEN DISPLAY OF THE ORTEP ILLUSTRATION
}

The screen drawing subroutines available in ORTEP-III use PGPLOT. PGPLOT is a free graphics library developed by T. J. Pearson at the California Institute of Technology. The package is written in FORTRAN and operates on a variety of platforms. Information about PGPLOT can be found on the World Wide Web at http://astro.caltech.edu/ tjp/pgplot or via e-mail to tjp@astro.caltech.edu.

PGPLOT creates a window on the screen that represents an $81 / 2 \times 11$ inch drawing surface in landscape orientation on which ORTEP draws its illustration. After viewing the illustration, the user must click in the EXIT box with the mouse and hit RETURN to go back to the user input dialog. If the mouse does not operate, two RETURNs should work.

Users can interact with the illustration to identify atoms displayed on the screen. This is done by positioning the cursor (with a mouse or with the keyboard's arrow keys) on a displayed atom and clicking the mouse button or typing the letter " $a$ ". The atom's label (if it has one) and atom designator code are displayed. Whether a mouse will work depends on how PGPLOT operates on the particular platform. The cursor must be positioned within $1 / 16$ inch of the atom center. If ORTEP identifies two or more atom centers within $1 / 16$ inch of the cursor, it will provide the identification of the atom center closest to the cursor position along with question marks. Exercise caution when atoms lie directly over or very near each other.

\subsection{PLOTTING THE ORTEP ILLUSTRATIONS}

The destination of the ORTEP illustration is controlled by the value stored in the variable NDRAW, which is set from user input in subroutine UINPUT and subsequently held in COMMON NS. The pre-defined values for NDRAW built into ORTEP-III are listed below. Exercise caution if the illustration destination code is altered. If a new destination is needed, it is probably a good idea to create a new value for NDRAW that is different from those already programmed.

\author{
NDRAW $=0:$ no illustration \\ NDRAW $=1:$ screen output \\ NDRAW $=2$ : EPS file output \\ NDRAW = 3: HPGL file output \\ NDRAW = 9: RESERVED for future use
}


Seven subroutines control the plotting of illustrations produced by ORTEP-III-F200, PLOT, INIT $x x$, COLR $x x$, PEN $x x$, PENW $x x$, and END $x x$ [ $x x$ refers to a specific plotting device (see below)]. These may be modified to produce the output required to plot the illustrations on a variety of devices.

Subroutine F200 responds to 200 series instructions in the ORTEP input file. A 201 instruction produces a call to INIT $x x$, which contains the specific initialization code for device $x x$. Similarly, a 202 instruction with no parameters (i.e., blanks or "0"s) produces a call to END $x x$, which contains the device's termination code. When a 202 instruction with one or two non-zero parameters is received by F200, it interprets the parameters as the $x$ - and $y$-shift, respectively, in the plotting origin. These values are stored in the variables XTRANS and YTRANS, respectively, that are held in COMMON TRFAC. (These variables are both initially set to " 0 " when the 201 is received.) The 204 instruction produces a call to COLRxx to change the color of the plot. PENW $x x$ is called to change the thickness of the plotted lines in response to a 205 instruction. For compatibility with OR TEP- $\Pi^{2}$ the 203 instruction is treated as a 201.

The illustration is produced when ORTEP calls subroutine PLOT with the arguments $X$, $\mathrm{Y}$, and IPEN. $\mathrm{X}$ and $\mathrm{Y}$ are the $x, y$ position (in inches) where the plotting device's pen should move, and IPEN is a flag that specifies whether the pen should be down (producing a line, IPEN=2) or up (not producing a line, IPEN=3) as it moves to its new position. These values are sent to PEN $x x$, which contains the specific code for device $x x$ to handle the pen movements. The values of XTRANS and YTRANS are added to $X$ and $Y$, respectively, in PEN $x x$.

Two functional plotting "devices" are included in the ORTEP-III distribution. These do not actually control physical devices but instead create files containing EPS and HPGL/2 descriptions of the illustrations. These files may be downloaded to printers/plotters that accept those particular page description languages. ORTEP-III itself does not automatically download (or spool) the files for printing/plotting. Some computer graphics programs and word processors will import these files. The EPS specific codes are in subroutines INITPS, COLRPS, PENPS, PENWPS, and ENDPS. The HPGL specific codes are in subroutines INITHP, COLRHP, PENHP, PENWHP, and ENDHP.

Since every user will not have PGPLOT, the five subroutines that control screen drawing-INITSC, COLRSC, PENSC, PENWSC, and ENDSC - are not functional in the ORTEP-III source code distribution, i.e., they are "dummy" subroutines containing only the single FORTRAN instruction RETURN. However, on systems that have PGPLOT implemented, alternate code for these five subroutines is available in the distribution and should be substituted for the dummy versions. INITSC creates the window in which the ORTEP illustration is drawn. The size and orientation of the window can be changed by modifying the code in INITSC.

The PGPLOT screen driver code includes a subroutine named CURSSC that has no counterpart in the EPS and HPGL drivers. This code allows users to identify atoms displayed on the screen (see 4.2).

\subsection{INTERACTIVE EDITOR}

ORTEP-III provides a simple line editor for editing the input file without exiting the program. NOTE: Comments in the input file beginning with \# will not be displayed and will not be retained if the instruction set is saved. The editor cannot be used to create comments beginning with \#. (See 1.2.6.) 
and branches to subroutine READIN to read the information. This subroutine may be modified and the program recompiled to read any desired format.

Subroutine READIN has 16 parameters as described below. Upon entry, only IU, the input file unit number, has been set by the calling routine. All other parameters must be set in READIN before control is returned to the calling routine. The READIN subroutine distributed with ORTEP-III may be used to read small protein fragments (500 atoms or less) in the Brookhaven Protein Data Bank format. (See 7.4.)

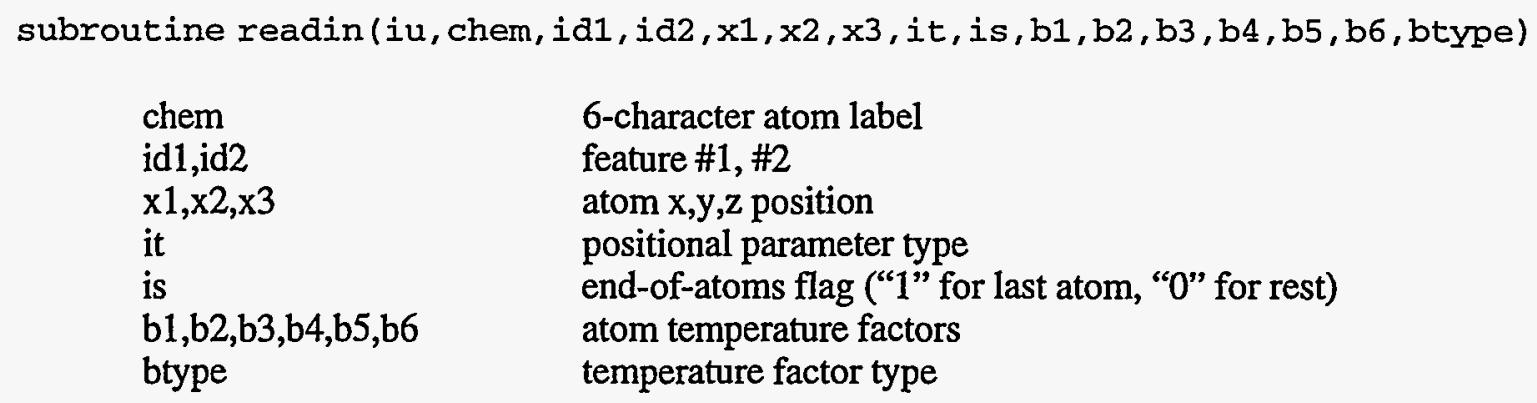

\subsection{ATOM 'FEATURES'}

In earlier versions of ORTEP, atoms could be referenced only by their numeric positions in the input file. Thus, atom number runs (ANR) (see 3.1.4) were used to select groups of atoms to be treated in the same manner. ORTEP-III allows two optional attributes called "features" (see 3.1.10) to be provided with each atom, and feature number runs (FNR) (see 3.1.11) can be used to select groups of atoms having particular features. Features should prove especially useful for polymeric materials such as proteins or nucleic acids as will be seen in the examples below.

The two atom features are stored in the $\operatorname{INTEGER} * 2$ variables $\operatorname{IDENT}(1, n)$ and $\operatorname{IDENT}(2, n)$ where $n$ is the atom number in the input list. The first of these contains Feature \#1 of the atom and the second contains Feature \#2. These can be assigned values as appropriate to the required task.

For typical ORTEP input, features can be entered in columns 10-18 and 19-27 on Type 0, 1 , and 2 atom positional parameter cards (see 3.2.4.1). The values (or zeroes) in these fields are read in and stored in temporary real variables. They are also truncated to integers and stored in $\operatorname{DENT}(1, n)$ and $\operatorname{IDENT}(2, n)$. If these fields happen to contain numeric values unrelated to feature definition, they will be ignored and will not interfere in any way with the operation of the program. However, if commands are invoked that specifically look at feature values, ORTEP will assume these numbers represent features of the atoms.

To illustrate this new concept, suppose a crystal contains four elements but the user wishes to plot only the atoms of one element, say sulfur. If all the sulfur atoms are grouped together in the input file, a single ANR would select them. If the sulfur atoms do not occur together in the input file, several ANRs would be needed to select all of them. In either case, the user would have to count the atoms in the input file to determine the starting and ending position numbers of the sulfur atoms. On the other hand, if the atomic number of each atom is recorded as Feature \#1 in the input list, a single FNR to find all atoms with the value 16 for Feature \#1 would select all sulfur atoms regardless of their positions in the input list. 
The most common use for features will probably be with macromolecules. For example, in a protein, a feature field can be a convenient place to store a residue number or a code representing a structural feature of an amino acid. Atom parameters for these large molecules are likely to be read into ORTEP with the subroutine READIN (see 4.5) In this case, code can be written in READIN that sets the values of $\operatorname{IDENT}(1, n)$ and $\operatorname{IDENT}(2, n)$ directly. Another place where features can be useful is in critical net illustrations (see 7.5) where it is advantageous to distinguish the peak, pass, pale, and pit critical points.

To handle features, a new parameter, number run type (see 3.1.12), has been added to the 100 series, 400 series, 505, 506, 700 series, 800 series, and 1001 instructions. See the instruction descriptions in Section 3 for the details.

Example: A protein contains 60 amino acids with a total of 500 atoms. Feature \#1 of each atom has the sequence number of the amino acid containing the atom. Feature \#2 has codes for the structural characteristics of the atom: 1 for an $\alpha$ carbon, 2 for a $\mathrm{C}=\mathrm{O}$ carbon in the peptide link, 3 for a nitrogen in the peptide link, 4 for all other atoms.

- To select all the atoms in amino acids 9-17:

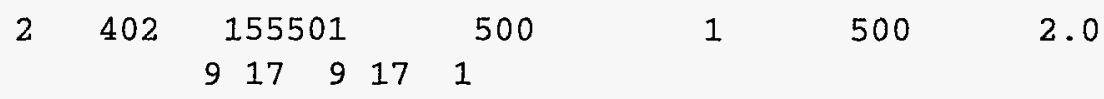

- To select all the atoms in the protein backbone:

$4021^{155501} 31$ 3 $2^{500}$

1500

2.0

- To select all the backbone atoms in amino acids 9-17:

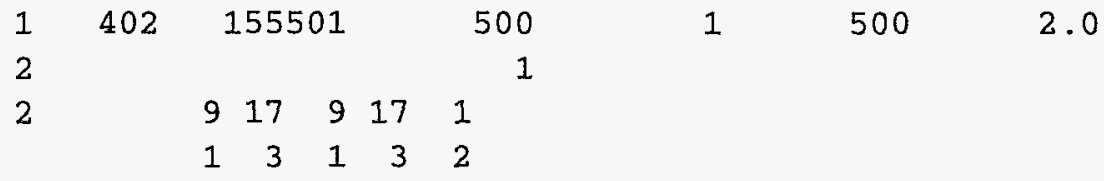

- To draw all atoms in the protein:

$1 \quad 712$

1500

- To draw the atoms in the protein backbone:

$1 \quad 712$

- To draw the atoms in amino acids 9-17: 
- To draw the bonds between the atoms in amino acids 9-17:

$2 \quad 812$

$9 \begin{array}{llllllll} & 17 & 9 & 17 & 1 & 0.9 & 2.0 & .04\end{array}$

- To correct for overlap among the atoms and bonds in amino acids 9-17:

21001

1

$\begin{array}{lllllllll}9 & 17 & 9 & 17 & 1 & 0.9 & 2.0 & .04\end{array}$

\subsection{MODIFYING ORTEP-III}

Appendix $A$ is a listing and brief description of the subprograms that make up ORTEPIII, and Appendix B has the same information for the major variables that are used. The entire FORTRAN program listing is in Appendix C. This information is provided to help users who wish to make changes to the program. 
.

$:$ 


\section{TECHNICAL DETAIIS}

Publications by Heading, ${ }^{8}$ Springer, ${ }^{9}$ Todd, ${ }^{10}$ and Korn and Korn ${ }^{11}$ provide background on many of the projective and analytical geometry concepts discussed in this section.

\subsection{HOW ORTEP DRAWS ELLIPSOIDS}

Fig. 3.1 illustrates various ellipsoid graphical representations that can be drawn with ORTEP. The major components in the representations are the three principal ellipses and the boundary (outline) ellipse. The principal ellipses have a front half and a back (hidden) half. The entire boundary ellipse is visible.

An ellipse is approximated by connecting a series of points on the ellipse with straight line segments. Points on an ellipse having a general orientation in three dimensions are computed; then each of these points is projected onto the drawing board for plotting.

The basic algorithm for finding the points along a given general ellipse utilizes the properties of conjugate diameters. Assume that we have the three principal axis vectors $\mathbf{V}_{1}, \mathbf{V}_{2}, \mathbf{V}_{3}$ of the general ellipsoid and a vector $\mathbf{V}_{\mathbf{4}}$ from the center of the ellipsoid to the viewpoint. The vector $\mathbf{V}_{5}$ normal to the polar plane (see Fig. 5.1), whose pole is the viewpoint, can be obtained from

$$
\mathbf{V}_{5}=\mathrm{A} \mathbf{V}_{4}
$$

where $\mathbf{A}$ is the matrix for the ellipsoid that is defined by

$$
\mathbf{X}^{\mathrm{T}} \mathbf{A} \mathbf{X}=d,
$$

where $d$ is a constant.

The boundary ellipse is defined by two conjugate vectors, one of which is any vector $\mathbf{V}_{6}$ perpendicular to $V_{5}$ and the second is $V_{7}$, where

$$
V_{7}=V_{5} \times A V_{6}
$$

The assumption made for this boundary ellipse derivation is that the view distance is large compared to the ellipsoid size. Therefore, the boundary ellipse defined above always lies on the diametral polar plane (see Fig. 5.1).

A principal ellipse that lies in the plane of the principal axis vectors $\mathbf{V}_{1}$ and $\mathbf{V}_{\mathbf{2}}$ will have the third principal axis vector $V_{3}$ normal to the plane of the ellipse. The intersection of this principal ellipse with the boundary ellipse is along the vector $V_{8}$ where

$$
v_{8}=v_{5} \times v_{3}
$$

This vector divides the front and back (hidden) sides of the principal ellipse. A vector conjugate to $V_{8}$ and in the principal plane containing $V_{1}$ and $V_{2}$ is $V_{9}$, where

$$
V_{9}=V_{3} \times A V_{8}
$$




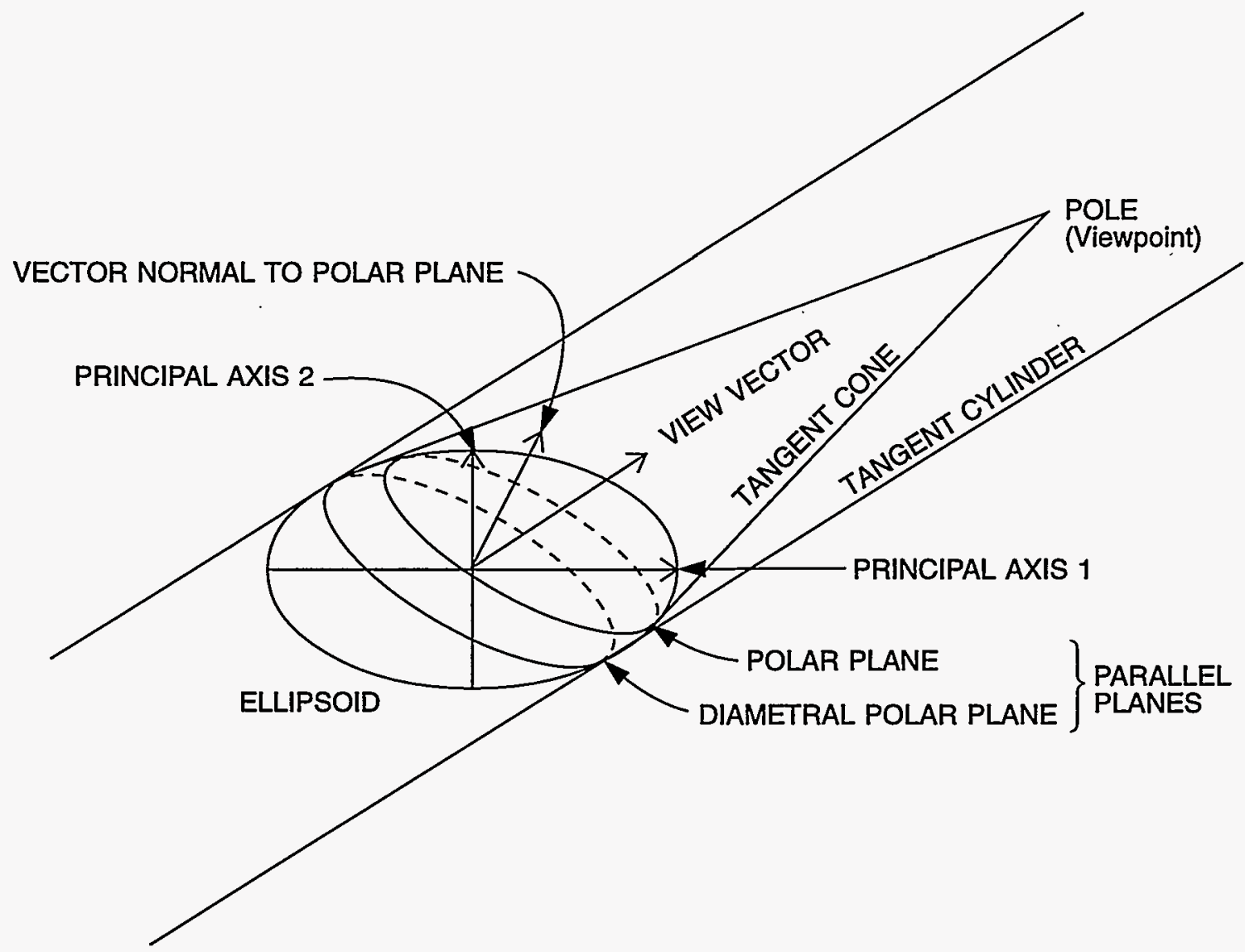

Fig. 5.1. Polar planes formed by tangent cylinder and tangent cone.

After the conjugate vectors have been found, their lengths are adjusted to make them satisfy Eq. 5.1 .2 by letting $X=s I$, where $I$ is a unit vector. Solving for $s$, we obtain

$$
s=\left[d /\left(\mathbf{I}^{\mathrm{T}} \mathbf{A}\right)\right]^{1 / 2} .
$$

A conjugate vector pair is expanded into an ellipse by subroutine RADIAL. Since an ellipse is centrosymmetric, the two conjugate vectors and their negatives give four vectors whose endpoints lie on the ellipse. By performing a vector sum of two adjacent vectors and dividing the resultant vector components by $\sqrt{2}$, we can obtain an additional vector. After doing this for all adjacent pairs, we then have a total of eight vectors. This process can be repeated as many times as desired except that the scaling constant will be different for each cycle. The constant is described by

$$
\operatorname{CONST}_{i}=\sqrt{2\left[1+\cos \left(\pi / 2^{i}\right)\right]}=2 \cos \left(\pi / 2^{i+1}\right),
$$

where $i$ is the cycle number.

This total process may be thought of as taking a planar radial set of equally spaced unit vectors and performing a deformation and scaling on the space in which it is described. In geometry, this deformation is called an affine transformation. 
Complete details on drawing ellipsoids can be obtained from the FORTRAN coding of subroutines F700 and RADIAL.

\subsection{ELLIPSE RESOLUTION}

With printer/plotter resolution improvements, highly complex stereo drawings with each image of the pair about 2.25 inches wide can now be produced directly without photographic reduction. As a result, it has become necessary to adjust the resolution (smoothness) of the plotted elliptical curves in ORTEP to take full advantage of the output devices' capabilities.

ORTEP produces its ellipses by "stretching" an equal-area circle to the shape of the ellipse. (Since ORTEP draws all its shapes with straight lines, the circle is, in fact, a polygon, and the smoothness of the ellipse depends on the number of vertices, or spokes, in the polygon.) ORTEP can produce circles having $16,32,64$, and 128 spokes. The selection is a function of the radius of the circle. The default radii for changing to circles with fewer spokes are 0.09375 in. $(128 \rightarrow 64), 0.375 \mathrm{in} .(64 \rightarrow 32)$, and 0.75 in. $(32 \rightarrow 16)$. These default values are smaller than those in OR TEP-II. ${ }^{2}$

The smoothness of ellipses can be altered with the new 304 instruction in ORTEP-III, which allows the user to enter a smoothness factor. A factor of 1 sets the circle radii for changing to other spoke angles to the default values given above. A factor less than 1 produces smoother ellipses, and values greater than 1 produce ellipses with more "jaggies". If the factor is 0 , all ellipses, regardless of their size, will be drawn from circles having 128 spokes.

Fig. 5.2 shows three groups of ellipses produced with smoothness factor settings of 3,2 , and 0 . Although perfectly smooth ellipses are the ideal, it may be necessary to balance their appearance against the computational time to produce them and the sizes of the resultant files containing the ORTEP illustration. Table 5.1 shows the sizes of the EPS and HPGL files of the concentric ellipses in Fig. 5.2 as a function of the smoothness factor. Computational time was not determined for these examples, but it may become a significant factor on slower computers.

Table 5.1. Size of ORTEP illustration files as a function of ellipse smoothness factor settings.

\begin{tabular}{|c|c|c|}
\hline $\begin{array}{c}\text { Smoothness } \\
\text { Factor }\end{array}$ & $\begin{array}{c}\text { Size of } \\
\text { EPS File }\end{array}$ & $\begin{array}{c}\text { Size of } \\
\text { HPGL File }\end{array}$ \\
\hline 0. & 35544 & 28558 \\
\hline 0.5 & 29400 & 23566 \\
\hline 1. & 21464 & 17118 \\
\hline 2. & 11992 & 9422 \\
\hline 3. & 8408 & 6510 \\
\hline 5. & 7640 & 5886 \\
\hline 10. & 5848 & 4430 \\
\hline
\end{tabular}



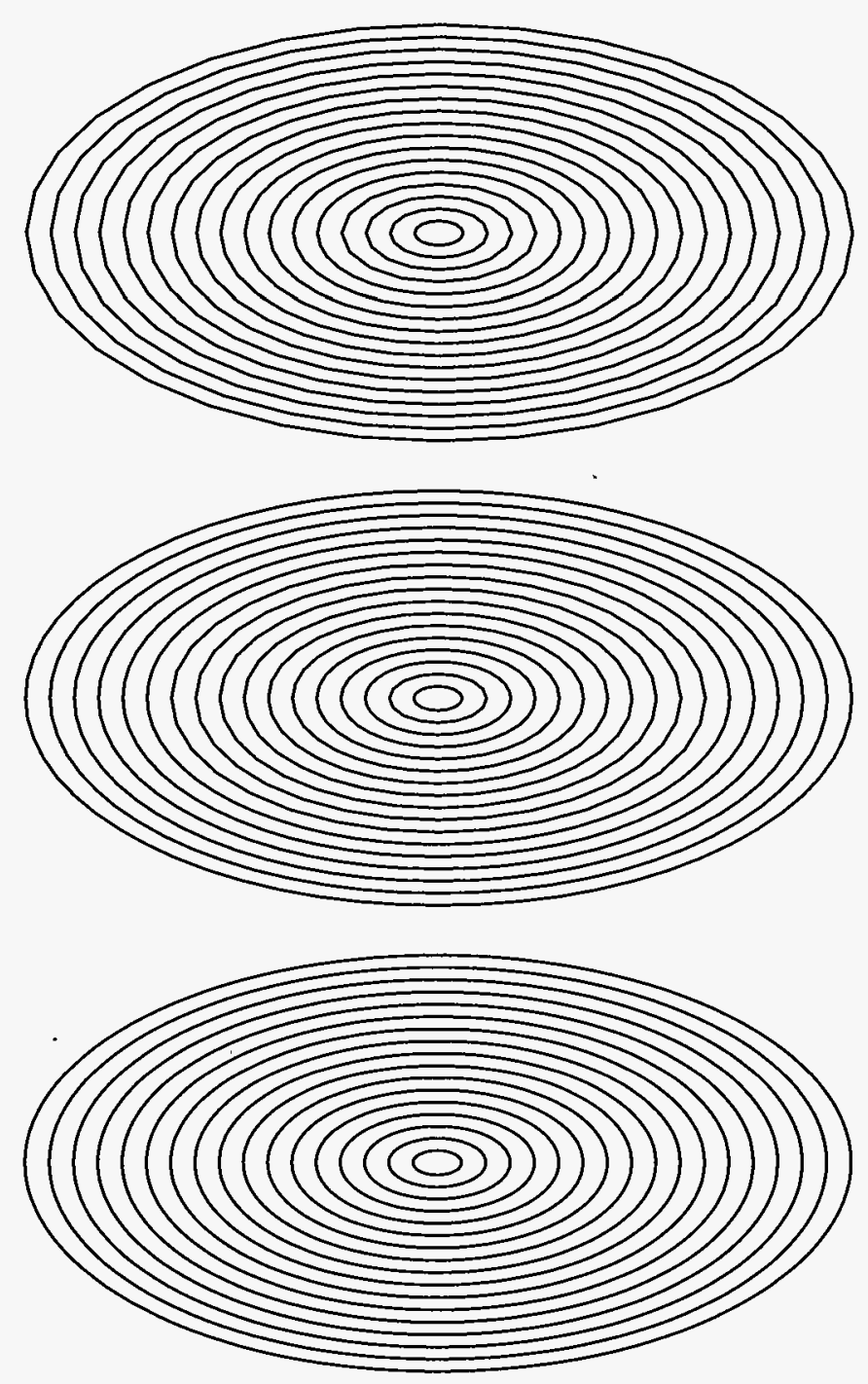

Fig. 5.2. Three sets of concentric ellipses produced by ORTEP with smoothness factor settings of 3,2 , and 0 , respectively, starting from the top.

\subsection{HOW ORTEP DRAWS BONDS}

The major difficulty when drawing bonds is obtaining the intersection where the bond penetrates the ellipsoid. Three quadrics are used in subroutine BOND to calculate bond intersection. These three are the ellipsoid, the tangent cylinder, and the tangent cone.

The ellipsoid is described in matrix notation as

$$
\mathbf{X}^{\mathrm{T}} \mathbf{A} \mathbf{X}=d
$$

where $d$ is a constant and $\mathbf{X}$ is any vector from the center to the surface of the ellipsoid. The matrix $\mathrm{A}$ is $3 \times 3$ symmetrical with components $a_{i j}(i, j=1,2,3)$. 
The elliptic cylinder tangent to the ellipsoid and with its axis along $z$ is described by

$$
\mathbf{X}^{\mathbf{T}} \mathbf{B} \mathbf{X}=d,
$$

where

$$
\mathbf{B}=\left(\begin{array}{ccc}
a_{11}-\frac{a_{13} a_{31}}{a_{33}} & a_{12}-\frac{a_{23} a_{31}}{a_{33}} & 0 \\
a_{12}-\frac{a_{13} a_{32}}{a_{33}} & a_{22}-\frac{a_{23} a_{32}}{a_{33}} & 0 \\
0 & 0 & 0
\end{array}\right)
$$

and $d$ is the constant used in Eq. 5.3.1. The tangent cylinder is used when it is necessary to terminate the bond at the boundary of the ellipsoid when a parallel projection is used.

To find the intersection of a cylindrical bond along $V_{b}$ with radius $r$ with either the ellipsoid or the tangent cylinder, we proceed as follows:

1. Form a radial set of vectors $\mathbf{V}_{r_{j}}$ of length $r$ normal to $\mathrm{V}_{\mathbf{b}}$.

2. Take a unit vector $\mathbf{I}$ parallel to $\mathrm{V}_{\mathrm{b}}$ and let

$$
\mathbf{X}_{j}=\mathbf{V r}_{j}+s \mathbf{I},
$$

where $s$ is a constant to be determined. Substituting in Eq. 5.3.1, we obtain

$$
s^{2} I^{\mathrm{T}} \mathbf{A} I+2 s V_{r}^{\mathrm{T}} \mathbf{A} I+V_{r}^{\mathrm{T}} \mathrm{A} V_{r}-d=0 ;
$$

and solving for $s$, we get

$$
s=\frac{-V_{r}{ }^{\mathrm{T}} \mathbf{A} I+\sqrt{\left(\mathbf{V r}^{\mathrm{T}} \mathbf{A} \mathbf{I}^{2}-\left(\mathbf{I}^{\mathrm{T}} \mathbf{A I}\right)\left(\mathbf{V r}^{\mathrm{T}} \mathbf{A} V_{r}-d\right)\right.}}{\mathbf{I}^{\mathrm{T}} \mathbf{A I}} .
$$

The elliptic cone that is tangent to the ellipsoid and has its apex on the viewpoint can be obtained from the matrix $A$ and the vector $V u$, which extends from the center of the ellipsoid to the viewpoint. This is performed in the following steps:

1. The ellipsoid is transformed with a rotation matrix to a new Cartesian frame of reference that has the $z$ axis along the view vector $V u$.

2. The tangent cone can now be described as

$$
\mathbf{Y}^{\mathrm{T}} \mathbf{C Y}=\mathbf{0}
$$

where $\mathrm{Y}$ is a vector originating from the vertex (viewpoint) of the cone and 


$$
\mathbf{C}=\left(\begin{array}{ccc}
a_{11}+\frac{a_{13} a_{31}}{K-a_{33}} & a_{12}+\frac{a_{13} a_{32}}{K-a_{33}} & \frac{K a_{13}}{K-a_{33}} \\
a_{21}+\frac{a_{23} a_{31}}{K-a_{33}} & a_{22}+\frac{a_{23} a_{32}}{K-a_{33}} & \frac{K a_{23}}{K-a_{33}} \\
\frac{K a_{31}}{K-a_{33}} & \frac{K a_{32}}{K-a_{33}} & \frac{K a_{33}}{K-a_{33}}
\end{array}\right), K=d /\left(\mathrm{Vu}^{\mathrm{T}} \mathrm{Vu}_{\mathrm{u}}\right)
$$

3. The frame of reference is rotated back to its original orientation with a rotation matrix that is the inverse of the one used in step 1. Note that the origin is now on the viewpoint rather than on the ellipsoid center.

To find the length, $s$, of a vector $s I$ extending from any point $p$ inside the cone to the surface of the cone, we let

$$
\mathbf{Y}=\mathbf{V}_{\mathbf{p}}+s \mathbf{I}
$$

and obtain from Eq. 5.3.7

$$
\left(\mathrm{V}_{\mathbf{p}}+s \mathbf{I}\right)^{\mathrm{T}} \mathbf{C}\left(\mathrm{V}_{\mathrm{p}}+s \mathbf{I}\right)=0
$$

then solving for $s$, we obtain

$$
s=\frac{-V_{p}^{T} C I+\sqrt{\left(V_{p}^{T} C I\right)^{2}-\left(I^{T} C I\right)\left(V_{p}^{T} C V_{p}\right)}}{I^{T} C I}
$$

The vector $V_{p}$ from the vertex to $p$ is formed by

$$
V_{p}=-V_{u}+V_{r},
$$

where $V_{r}$ is any member of a radial set such as that described for the regular ellipsoid intersection.

\subsection{OPTIMAL PARAMETERS FOR STEREOSCOPIC DRAWINGS}

For optimal viewing of stereoscopic drawings, the origins of the two views should be separated by $2.2-2.4$ in. and the stereo rotation between the two should be $5^{\circ}-6^{\circ}$.

Fig. 5.3 was created when it was common practice to produce "large" ORTEP drawings that would be photographically reduced to give the optimal origin separation. ${ }^{1,2}$ The reduced drawings would then typically be viewed with a stereoscope. Under these circumstances, Fig. 5.3 provides a picture of the relationship among the various parameters that must be taken into consideration when producing the drawing. ${ }^{12,13}$ 


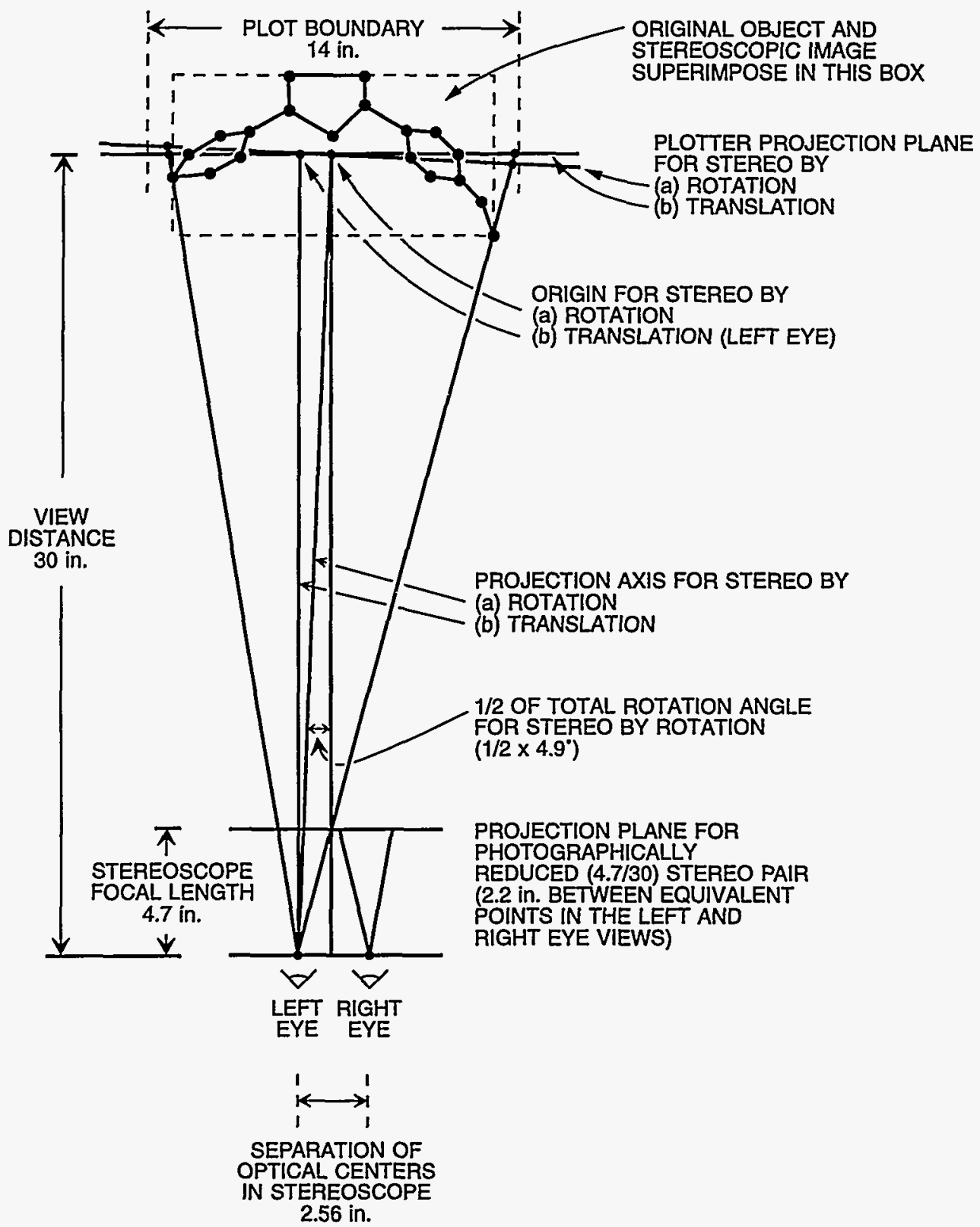

Fig. 5.3. Geometrical relations among the stereoscopic perspective projection parameters for a typical ORTEP drawing.

In Fig. 5.3, the available plotting area for each projection is assumed to be $14 \mathrm{in.} \mathrm{horizon-}$ tally and at least 11 in. vertically. The scaled mathematical object is within a box 12 in. wide, 9 in. (or more) high, and 6 in. deep with the plane of the plotter halfway back into the box. The stereoscopic image seen through a stereoscope with a 4.7-in. focal length and a 2.56-in. separation between optical centers should appear superimposed on the original object. The parameters for both "translation stereo" and "rotation stereo" are shown. The appropriate linear dimensions can be scaled to accommodate other plotting areas and still produce the same final stereoscopic image. 
With the advent of high resolution printers/plotters, the need to reduce large drawings has diminished, and high quality drawings with the optimal origin separation can be produced directly. For those who view such drawings without the aid of a stereoscope, the viewing distance set in ORTEP should be the actual physical distance that will be used to view the pair. If a stereoscope is used for viewing, the viewing distance set in ORTEP is a function of the device's focal length and the location of the drawing's origin.

The cubane example in Section 2.4 can be used as a tool for determining the optimal view distance parameter. When the stereo cubane drawing is observed under usual viewing conditions, users should compare the front and back faces to decide if the molecule actually looks like a cube. If the faces appear out of proportion with each other, adjust the view distance parameter until the cube looks correct.

Theoretically, the best stereoscopic fidelity is obtained by translation of the origin rather than by stereo rotation of the object; however, the comparison of results given in Fig. 5.4 shows that the differences are indeed minor and nearly impossible to detect.

A
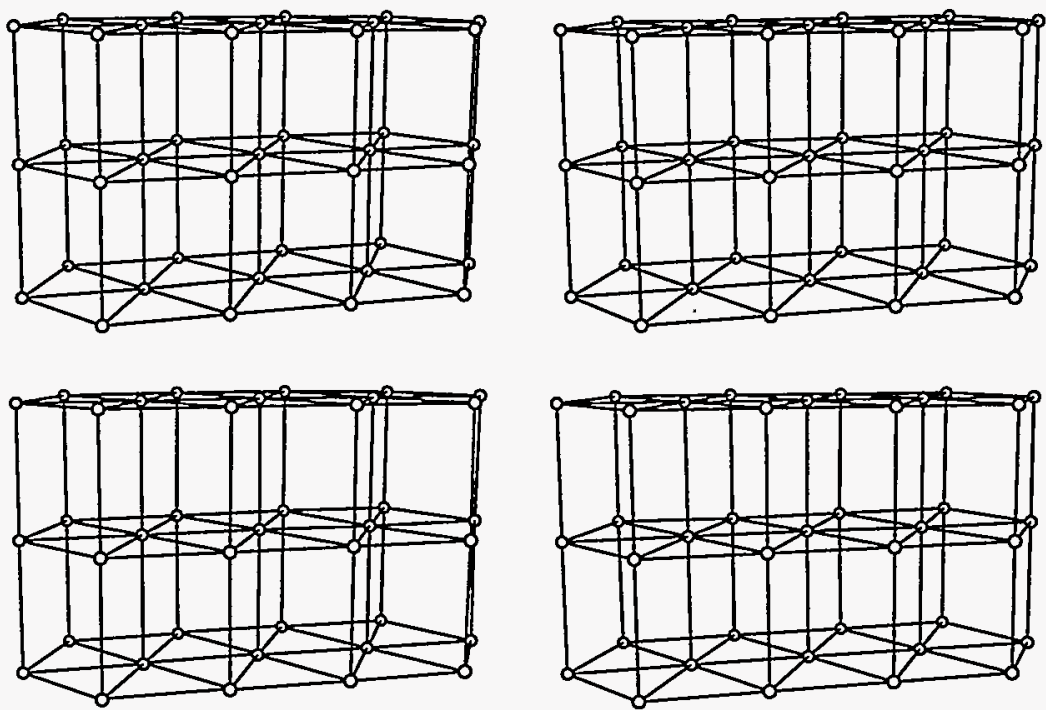

B
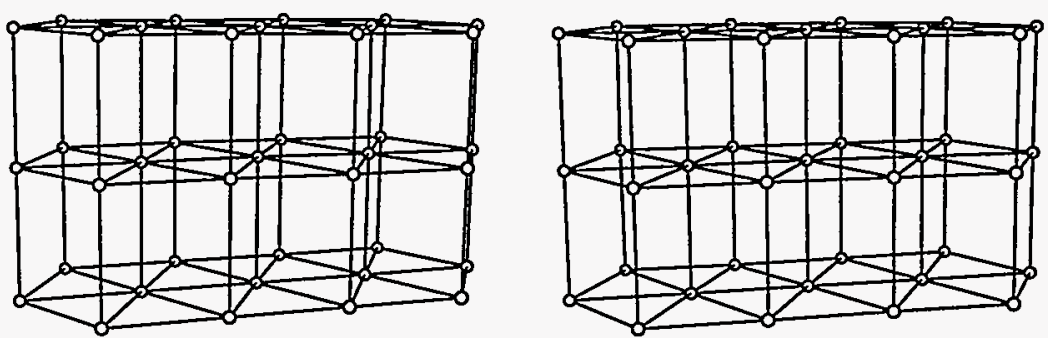

Fig. 5.4. Stereoscopic drawings of a hexagonal lattice with different stereoscopic parameters. (A) Stereo rotation of $4.9^{\circ}$, (B) stereo translation of 2.56 in., and (C) stereo rotation of $6.0^{\circ}$.

The top and middle drawings of Fig. 5.4 utilize the parameters derived in Fig. 5.3 and demonstrate that the differences predicted ${ }^{13}$ for translation and rotation stereo are not discernible 
in practice. The bottom stereo drawing illustrates the slight exaggeration in depth that occurs when a larger stereo rotation angle is used.

Fig. 5.4A was produced with the following stereo-rotation instructions for the left and right eye views,

$\begin{array}{lll}503 & 2 & 2.45 \\ 503 & 2 & -2.45\end{array}$

and stereo translation instructions were used for Fig. 5.4B.

$\begin{array}{rrrr}504 & -1.28 & 0 & 0 \\ 504 & 2.56 & 0 & 0\end{array}$

Remember that the 504 instruction (see 3.3.6.4) changes the origin of the reference Cartesian system while the 503 instruction (see 3.3.6.3) rotates the working Cartesian system. If additional drawings are to be made following a stereo translation, the reference system origin should be returned to its original position in order to prevent confusion.

$\begin{array}{llll}504 & -1.28 & 0 & 0\end{array}$

Also keep in mind that the 504 instruction (see 3.3.6.4) should not be used when the ellipsoids have internal structure because the octants selected for shading may not be the same in both views. 


\section{MATHEMATICS OF THERMAL-MOTION PROBABILITY ELLIPSOIDS}

It is convenient to develop the physical significance of the anisotropic temperature factor with the notation and terminology of probability theory rather than with the more familiar Fourier transform theory. The results are, of course, identical regardless of the terminology used. The reason for this choice is that the literature of mathematical statistics and probability theory is somewhat neater and easier to follow. The texts by Wilks, ${ }^{14}$ Cramer, ${ }^{15}$ Miller, ${ }^{16}$ Hamilton, ${ }^{17}$ and Lukacs and Laha ${ }^{18}$ and the handbooks by Burington and May ${ }^{19}$ and Owen ${ }^{20}$ are found to be particularly useful.

\subsection{PROBABILITY DENSITY FUNCTION OF A TRIVARIATE NORMAL DISTRIBUTION}

Given three chance variables $X_{1}, X_{2}, X_{3}$ and $S$, which is a region in $X_{1}, X_{2}, X_{3}$ space, the probability $P(S)$ that the point $\left(X_{1}, X_{2}, X_{3}\right)$ falls in the region $S$ is given by

$$
P(S)=\iint_{S} \int \phi\left(X_{1}, X_{2}, X_{3}\right) d X_{1} d X_{2} d X_{3}
$$

If the integration is carried over all space, then

$$
\iint_{-\infty}^{\infty} \int \phi\left(X_{1}, X_{2}, X_{3}\right) d X_{1} d X_{2} d X_{3}=1
$$

The function $\phi\left(X_{1}, X_{2}, X_{3}\right)$ is called the probability density function (pdf) for the joint distribution of $X_{1}, X_{2}, X_{3}$. Using vector notation, we can designate the pdf as $\phi(X)$.

When the distribution is the type said to be normal or Gaussian, the pdf is

$$
\phi(X)=\frac{\left[\operatorname{det}\left(M^{-1}\right)\right]^{1 / 2}}{(2 \pi)^{3 / 2}} \exp \left[-\frac{1}{2}\left(\mathbf{X}-\hat{\mathbf{X}}^{\mathrm{T}} \mathbf{M}^{-1}(\mathbf{X}-\hat{\mathbf{X}})\right] .\right.
$$

The matrix $M^{-1}$ is the inverse of the symmetrical dispersion (variance-covariance) matrix $M$, where

$$
\mathbf{M}=\left(\begin{array}{lll}
\sigma_{1}^{2} & \sigma_{1} \sigma_{2} \rho_{12} & \sigma_{1} \sigma_{3} \rho_{13} \\
\sigma_{1} \sigma_{2} \rho_{12} & \sigma_{.2}^{2} & \sigma_{2} \sigma_{3} \rho_{23} \\
\sigma_{1} \sigma_{3} \rho_{13} & \sigma_{2} \sigma_{3} \rho_{23} & \sigma_{3}^{2}
\end{array}\right)
$$

The symbols $\sigma_{i}^{2}$ represent the second moments or variance about the mean position $\hat{\mathbf{X}}$. The symbols $\sigma_{i} \sigma_{j} \rho_{i j}$ are the corresponding covariances and $\rho_{i j}$ are the correlation coefficients. 


\subsection{EQUIPROBABILITY ELLIPSOIDS}

For a proper normal distribution, the quadratic form $(\mathbf{X}-\hat{\mathbf{X}})^{\mathrm{T}} \mathbf{M}^{-1}(\mathbf{X}-\hat{\mathbf{X}})$ is positive definite, and a principal axis transformation (see 6.4) is possible that will make the cross correlation coefficients $\rho_{i j}=0(i \neq j)$. The result of the transformation is the pdf

$$
\phi\left(y_{1}, y_{2}, y_{3}\right)=\frac{1}{(2 \pi)^{3 / 2} \sigma_{y_{1}} \sigma_{y_{2}} \sigma_{y_{3}}} \mathrm{e}^{-Q / 2},
$$

where

$$
Q=\frac{\left(y_{1}-\hat{y}_{1}\right)^{2}}{\sigma_{y_{1}}^{2}}+\frac{\left(y_{2}-\hat{y}_{2}\right)^{2}}{\sigma_{y_{2}}^{2}}+\frac{\left(y_{3}-\hat{y}_{3}\right)^{2}}{\sigma_{y_{3}}^{2}}
$$

The $y_{i}$ are coordinates based on the Cartesian principal axis system and $\sigma_{y_{i}}^{2}$ are the variances
along the principal axes, $i=1,2,3$.

The normal probability density function is constant for points on the ellipsoid $Q=C^{2}$ where $C$ is a constant. The probability that a random point $\left(y_{1}, y_{2}, y_{3}\right)$ in the distribution will fall inside the ellipsoid is

$$
P(C)=(2 / \pi)^{1 / 2} \int_{0}^{C} r^{2} \mathrm{e}^{-r^{2} / 2} d r
$$

This result is derived from Eqs. 6.1.1, 6.2.1, and 6.2.2 by transforming to spherical coordinates.

When $C=1.5382, P=0.5$ and the corresponding ellipsoid is called the $50 \%$ probability ellipsoid. Table 6.1 is a table of $P$ versus $C$ values that were calculated by integrating Eq. 6.2.3 using Gaussian quadrature. Quadruple precision calculations were required to match the values found on page 203 of Owen's handbook. ${ }^{20}$

\subsection{CHARACTERISTIC FUNCTION OF A TRIVARIATE NORMAL DISTRIBUTION}

The characteristic function $\Phi(T)$ corresponding to a trivariate distribution $\phi(X)$ is the expected value of $\mathrm{e}^{i \mathrm{~T}^{\top} \mathrm{x}}$, namely,

$$
\Phi(\mathbf{T})=\int_{-\infty}^{\infty} \phi(\mathbf{X}) \mathrm{e}^{i \mathbf{T}^{\mathbf{T} X}} d \mathbf{X}
$$

For the trivariate normal pdf, Eq. 6.1.3, the corresponding characteristic function is

$$
\Phi(\mathbf{T})=\exp \left[i \mathbf{T}^{\mathrm{T}} \hat{\mathbf{X}}-\frac{1}{2} \mathbf{T}^{\mathrm{T}} \mathbf{M} \mathbf{T}\right]
$$

where $M$ is the variance-covariance dispersion matrix described in Section 6.1 and $\hat{X}$ is the center of mass of the distribution. 
Table 6.1. Critical values for probability ellipsoids of a trivariate normal distribution.

\begin{tabular}{|c|c|c|c|c|c|}
\hline $\mathrm{P}$ & C & $P$ & $C$ & $P$ & C \\
\hline 0.01 & 0.3389 & 0.41 & 1.3842 & 0.81 & 2.1824 \\
\hline 0.02 & 0.4299 & 0.42 & 1.4013 & 0.82 & 2.2114 \\
\hline 0.03 & 0.4951 & 0.43 & 1.4183 & 0.83 & 2.2416 \\
\hline 0.04 & 0.5479 & 0.44 & 1.4354 & 0.84 & 2.2730 \\
\hline 0.05 & 0.5932 & 0.45 & 1.4524 & 0.85 & 2.3059 \\
\hline 0.06 & 0.6334 & 0.46 & 1.4695 & 0.86 & 2.3404 \\
\hline 0.07 & 0.6699 & 0.47 & 1.4866 & 0.87 & 2.3767 \\
\hline 0.08 & 0.7035 & 0.48 & 1.5037 & 0.88 & 2.4153 \\
\hline 0.09 & 0.7349 & 0.49 & 1.5209 & 0.89 & 2.4563 \\
\hline 0.10 & 0.7644 & 0.50 & 1.5382 & 0.90 & 2.5003 \\
\hline 0.11 & 0.7924 & 0.51 & 1.5555 & 0.91 & 2.5478 \\
\hline 0.12 & 0.8192 & 0.52 & 1.5729 & 0.92 & 2.5997 \\
\hline 0.13 & 0.8447 & 0.53 & 1.5904 & 0.93 & 2.6571 \\
\hline 0.14 & 0.8694 & 0.54 & 1.6080 & 0.94 & 2.7216 \\
\hline 0.15 & 0.8932 & 0.55 & 1.6257 & 0.95 & 2.7955 \\
\hline 0.16 & 0.9162 & 0.56 & 1.6436 & 0.96 & 2.8829 \\
\hline 0.17 & 0.9386 & 0.57 & 1.6616 & 0.97 & 2.9912 \\
\hline 0.18 & 0.9605 & 0.58 & 1.6797 & 0.98 & 3.1365 \\
\hline 0.19 & 0.9818 & 0.59 & 1.6980 & 0.99 & 3.3682 \\
\hline 0.20 & 1.0026 & 0.60 & 1.7164 & 0.991 & 3.4019 \\
\hline 0.21 & 1.0230 & 0.61 & 1.7351 & 0.992 & 3.4390 \\
\hline 0.22 & 1.0430 & 0.62 & 1.7540 & 0.993 & 3.4806 \\
\hline 0.23 & 1.0627 & 0.63 & 1.7730 & 0.994 & 3.5280 \\
\hline 0.24 & 1.0821 & 0.64 & 1.7924 & 0.995 & 3.5830 \\
\hline 0.25 & 1.1012 & 0.65 & 1.8119 & 0.996 & 3.6492 \\
\hline 0.26 & 1.1200 & 0.66 & 1.8318 & 0.997 & 3.7325 \\
\hline 0.27 & 1.1386 & 0.67 & 1.8519 & 0.998 & 3.8465 \\
\hline 0.28 & 1.1570 & 0.68 & 1.8724 & 0.999 & 4.0331 \\
\hline 0.29 & 1.1751 & 0.69 & 1.8932 & 0.9991 & 4.0607 \\
\hline 0.30 & 1.1932 & 0.70 & 1.9144 & 0.9992 & 4.0912 \\
\hline 0.31 & 1.2110 & 0.71 & 1.9360 & 0.9993 & 4.1256 \\
\hline 0.32 & 1.2288 & 0.72 & 1.9580 & 0.9994 & 4.1648 \\
\hline 0.33 & 1.2464 & 0.73 & 1.9804 & 0.9995 & 4.2107 \\
\hline 0.34 & 1.2638 & 0.74 & 2.0034 & 0.9996 & 4.2661 \\
\hline 0.35 & 1.2812 & 0.75 & 2.0269 & 0.9997 & 4.3365 \\
\hline 0.36 & 1.2985 & 0.76 & 2.0510 & 0.9998 & 4.4335 \\
\hline 0.37 & 1.3158 & 0.77 & 2.0757 & 0.9999 & 4.5943 \\
\hline 0.38 & 1.3330 & 0.78 & 2.1012 & 0.99999 & 5.0894 \\
\hline 0.39 & 1.3501 & 0.79 & 2.1274 & 0.999999 & 5.5376 \\
\hline 0.40 & 1.3672 & 0.80 & 2.1544 & 0.9999999 & 5.9503 \\
\hline
\end{tabular}


The crystallographic structure factor equation that incorporates general anisotropic temperature factor coefficients is

$$
F(\mathbf{h})=\sum_{n} f_{n}(\mathbf{h}) \exp \left(2 \pi i \mathbf{h}^{\mathrm{T}} \hat{\mathbf{X}}_{n}\right) \exp \left(-\mathbf{h}^{\mathrm{T}} \mathbf{B}_{n} \mathbf{h}\right)
$$

where

h is a vector giving the Miller indices,

$\mathbf{X}_{n}$ is a vector giving the fractional unit cell coordinates of the $n$th atom,

$\mathbf{B}_{n} \quad$ is the anisotropic temperature factor coefficient matrix, and

$f_{n}(h)$ is the atom form factor value for atom $n$.

If a change of variables $T=2 \pi h$ is made, then Eq. 6.3.3 can be rewritten as

$$
F(\mathbf{T})=\sum_{n} f_{n}(\mathbf{T}) \exp \left(i \mathbf{T}^{\mathrm{T}} \hat{\mathbf{X}}_{n}-\frac{1}{2} \mathbf{T}^{\mathrm{T}} \frac{\mathbf{B}}{2 \pi^{2}} \mathbf{T}\right)
$$

The scaled anisotropic temperature factor matrix $\left(1 / 2 \pi^{2}\right) \mathrm{B}$ is seen to be identical with the variance-covariance dispersion matrix $M$ in Eq. 6.3.2.

The corresponding crystal space trivariate normal pdf for any particular atom $n$ is

$$
\phi(\mathbf{X})=\frac{\left[2 \pi^{2} \operatorname{det}\left(\mathbf{B}^{-1}\right)\right]^{1 / 2}}{(2 \pi)^{3 / 2}} \exp \left[-\pi^{2}(\mathbf{X}-\hat{\mathbf{X}})^{\mathrm{T}} \mathbf{B}^{-1}(\mathbf{X}-\hat{\mathbf{X}})\right]
$$

or if $M^{-1}=2 \pi^{2} B^{-1}$, then

$$
\phi(\mathbf{X})=\frac{\operatorname{det}\left(\mathbf{M}^{-1}\right)}{(2 \pi)^{3 / 2}} \exp \left[-\frac{1}{2}(\mathbf{X}-\hat{\mathbf{X}})^{\mathbf{T}} \mathbf{M}^{-1}(\mathbf{X}-\hat{\mathbf{X}})\right]
$$

which is identical to Eq. 6.1.3.

\subsection{PRINCIPAL AXIS TRANSFORMATION}

The transformation of anisotropic temperature factor coefficients (for the general triclinic case) to principal axes of thermal motion is discussed by Waser, ${ }^{21}$ Busing and Levy, ${ }^{22}$ and Cruickshank et al. ${ }^{23}$

The principal axis transformation is necessary to find the thermal-motion probability ellipsoids discussed in Section 6.2. The principal axes of the matrix $\mathrm{M}^{-1}$ in Eq. 6.3.6 are the vectors $y_{1}$, $y_{2}, y_{3}$ for which the inner vector product $\left(y_{i}, y_{i}\right)$ has a stationary value subject to the constraint

$$
\left(\mathbf{y}_{i}, \mathbf{M}^{1} \mathbf{y}_{i}\right)=1, \quad i=1,2,3
$$

For the general triclinic crystal system, this means that the quadratic form $y^{T} G^{-1} y$ has a stationary value subjected to the constraint 


$$
y^{T} G^{-1} M^{-1} y=1
$$

where $G^{-1}$ is the metric tensor with components $a_{i} \cdot a_{j}$, where $a_{i} \cdot a_{j}$ is the scalar vector product of two of the three unit cell vectors. Introducing the Lagrange multiplier $1 / \lambda$ leads to

$$
\left[\mathbf{G}^{-1}-\frac{1}{\lambda_{i}} \mathbf{M}^{-1}\right] \mathbf{y}_{i}=0 \quad(i=1,2,3) ;
$$

premultiplying by $\mathbf{M}$ yields

$$
\left[\mathbf{M G}^{-1}-\frac{1}{\lambda_{i}} \mathbf{I}\right] \mathbf{y}_{i}=0 \quad(i=1,2,3)
$$

Or we can do some additional rearranging and obtain

$$
\left[\mathrm{GM}^{-1}-\lambda_{i} \mathrm{I}\right] \mathrm{y}_{i}=0 \quad(i=1,2,3) .
$$

Eq. 6.4.4 is equivalent to one of the results derived by Busing and Levy, ${ }^{22}$ except the $\lambda_{i}$ obtained here are the reciprocals of their $\lambda_{i}$ because we are doing the principal axis transformation on $M^{-1}$ while their formulation performs the transformation on $\mathbf{M}$. The numerical procedure used in ORTEP finds the eigenvalues and eigenvectors of the unsymmetrical matrix $\mathrm{MG}^{-1}$ in Eq. 6.4.4. 


\section{ORTEP EXAMPLES}

This section includes several example structures that illustrate a number of the capabilities found in ORTEP-III. The ORTEP input file for each example is provided.

\subsection{CELL PACKING - 5-HYDROXY-5-PHENYLNORBORNANONE}

Two illustrations of 5-hydroxy-5-phenylnorbornanone are provided here. The data were obtained from a neutron diffraction study at room temperature. ${ }^{24}$ The first illustration shows one complete molecule, and the second shows the contents of the unit cell.

The input file of the first structure illustrates ORTEP's original format for the symmetry operators. The atom parameter lines were taken directly from the output of a least squares refinement, and information is included there that is not needed by ORTEP. The extra information lies in card fields that are not required for ORTEP's operation and is ignored by the program. Note that the atoms are individually labeled with 901 instructions.

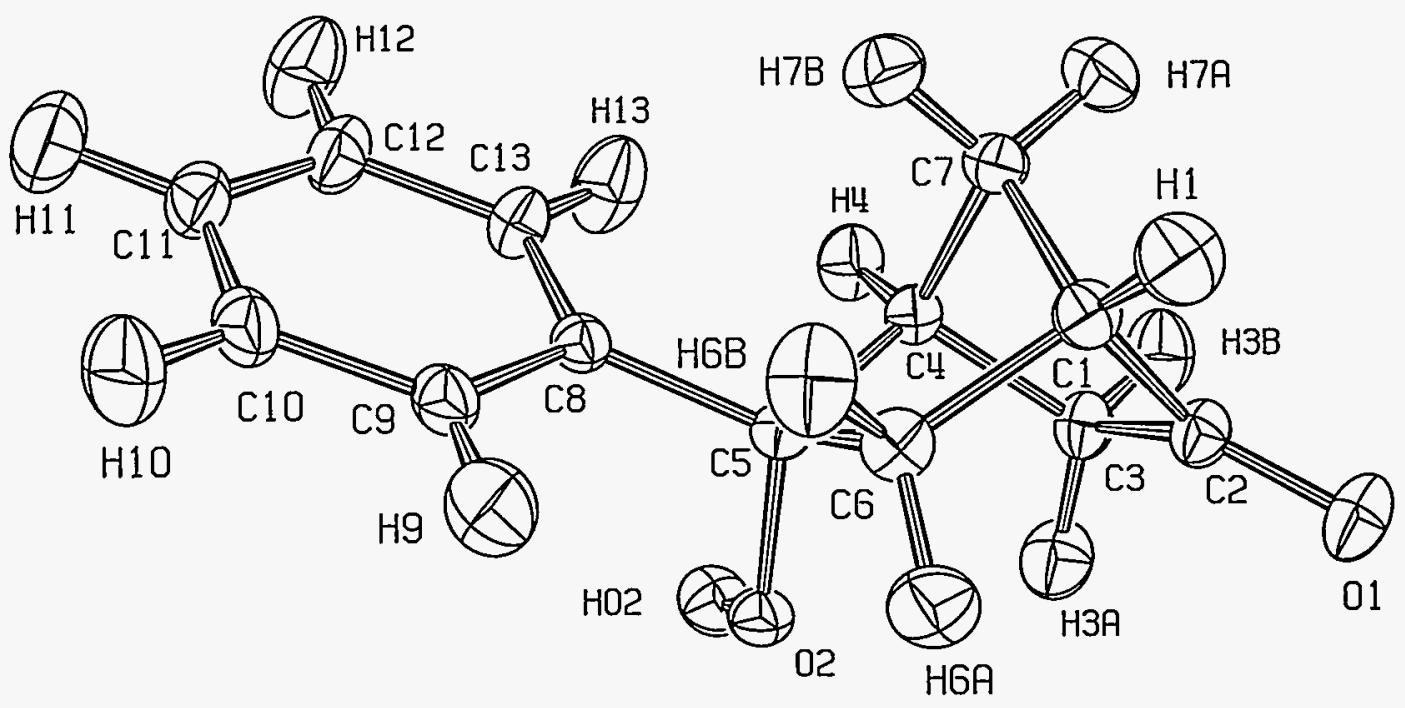

Fig. 7.1. Single molecule of 5-hydroxy-5-phenylnorbornanone.

\begin{tabular}{|c|c|c|c|c|c|c|c|c|c|c|c|c|c|}
\hline 10.331 & 10.64 & & 0.099 & 0.0 & -.28 & 8381 & & 0.0 & & & & & \\
\hline & .0 & 0 & 0 & & .0 & 0 & 1 & 0 & & .0 & 0 & 0 & 1 \\
\hline & $.0-3$ & 0 & 0 & & .0 & $0-7$ & 1 & 0 & & .0 & 0 & 0 & -1 \\
\hline & $.5-7$ & 0 & 0 & & .5 & 0 & 1 & 0 & & .5 & 0 & 0 . & -1 \\
\hline 1 & $.5:$ & 0 & 0 & & .5 & $0-$ & -1 & 0 & & .5 & 0 & 0 & 1 \\
\hline $\mathrm{C} 1$ & 0.66100 & 1. & 00000 & 0.224802 & 0.0 & 0163 & 80 & 0.901515 & 0.0 & & & & \\
\hline 0.011930 & 0.00675 & 0. & 11647 & 0.000642 & 0.0 & 0398 & & 0.000860 & & & 0 & 1 & 0 \\
\hline $\mathrm{C} 2$ & 0.66100 & 1. & 00000 & 0.335043 & 0.0 & 5084 & & 0.844329 & 0.0 & & & & \\
\hline 0.010432 & 0.00783 & 0.8 & 12112 & 0.001274 & 0.0 & $0394^{\prime \prime}$ & $7-0$ & 0.000264 & & & 0 & 1 & 0 \\
\hline C3 & 0.66100 & 1. & 00000 & 0.327553 & 0.1 & 9279 & & 0.850375 & 0.0 & & & & \\
\hline 0.009129 & 0.00788 & 0. & $13469-$ & 0.000262 & 0.0 & 0378 & & 0.000058 & & & 0 & 1 & 0 \\
\hline $\mathrm{C} 4$ & 0.66100 & 1. & 00000 & 0.206609 & 0.2 & 1347 & & 0.908171 & 0.0 & & & & \\
\hline 0.008568 & 0.00681 & 0.1 & $09641-$ & -0.000310 & 0.0 & 0211 & $18-0$ & 0.001032 & & & 0 & 1 & 0 \\
\hline
\end{tabular}




\begin{tabular}{|c|c|c|c|c|c|c|c|}
\hline C5 & 0.661000 & $1.000000 \quad 0.074610$ & 0.177193 & 0.796977 & 0.0 & & \\
\hline 0.008590 & 0.006732 & $0.008260-0.000670$ & 0.002360 & 0.000103 & & 0 & 1 \\
\hline c6 & 0.661000 & $1.000000 \quad 0.091094$ & 0.031659 & 0.790120 & 0.0 & & \\
\hline 0.011368 & 0.006963 & $0.012116-0.001403$ & $0.003143-$ & 0.001297 & & 0 & 1 \\
\hline C7 & 0.661000 & $1.000000 \quad 0.223679$ & 0.102901 & 1.010406 & 0.0 & & \\
\hline 0.011840 & 0.009377 & 0.0089810 .001423 & 0.002835 & 0.000648 & & 0 & 1 \\
\hline $\mathrm{C} 8$ & 0.661000 & $1.000000-0.053735$ & 0.216149 & 0.834431 & 0.0 & & \\
\hline 0.008328 & 0.007955 & $0.008995-0.000100$ & 0.002374 & 0.001216 & & 0 & 1 \\
\hline $\mathrm{Cg}$ & 0.661000 & $1.000000-0.175896$ & 0.157400 & 0.769638 & 0.0 & & \\
\hline 0.008481 & 0.012396 & $0.011267-0.001406$ & 0.001677 & 0.001394 & & 0 & 1 \\
\hline $\mathrm{C} 10$ & 0.661000 & $1.000000-0.296122$ & 0.195813 & 0.795042 & 0.0 & & \\
\hline 0.008526 & 0.016481 & $0.014906-0.000226$ & 0.002743 & 0.004954 & & 0 & 1 \\
\hline $\mathrm{c} 11$ & 0.661000 & $1.000000-0.295600$ & 0.294171 & 0.886394 & 0.0 & & \\
\hline 0.011357 & 0.014259 & $0.016607 \quad 0.003003$ & 0.006602 & 0.005712 & & 0 & 1 \\
\hline $\mathrm{C} 12$ & 0.661000 & $1.000000-0.175266$ & 2517 & 0.950755 & 0.0 & & \\
\hline 0.012936 & 0.012232 & 0.0184910 .002923 & 046 & 0.00 & & 0 & 1 \\
\hline C13 & 0.661000 & $1.000000-0.055392$ & 579 & 0.925104 & 0.0 & & \\
\hline 0.010921 & 0.009871 & $0.014343 \quad 0.000394$ & $5261-$ & 0.001410 & & 0 & 1 \\
\hline 01 & 0.577000 & $1.000000 \quad 0.414647-$ & & 0.801699 & 0.0 & & \\
\hline 0.014915 & 0.010743 & $0.020270 \quad 0.002523$ & $903-$ & 0.001350 & & 0 & 1 \\
\hline $\mathrm{O} 2$ & 0.577000 & $1.000000 \quad 0.068052$ & 607 & 0.664516 & 0.0 & & \\
\hline 0.010744 & 0.010771 & $0.008919-0.000196$ & 0.003244 & 0.001269 & & 0 & 1 \\
\hline $\mathrm{H} 1$ & -0.375000 & $1.000000 \quad 0.236869-$ & 5021 & 0.933996 & 0.0 & & \\
\hline 0.020624 & 0.008781 & 0.0191620 .002373 & 07156 & 0.003208 & & 0 & 1 \\
\hline H3A & -0.375000 & $1.000000 \quad 0.318029$ & 34292 & 0.749440 & 0.0 & & \\
\hline 0.015989 & 0.012191 & $0.019604 \quad 0.000563$ & 0.008507 & 0.003596 & & 0 & 1 \\
\hline $\mathrm{H} 3 \mathrm{~B}$ & -0.375000 & $1.000000 \quad 0.419746$ & 0.227078 & 0.923493 & 0.0 & & \\
\hline 0.011065 & 0.013144 & $0.022925-0.001035$ & 0.003225 & .0 .001808 & & 0 & 1 \\
\hline $\mathrm{H} 4$ & -0.375000 & $1.000000 \quad 0.205933$ & 0.307044 & 0.952030 & 0.0 & & \\
\hline 0.012786 & 0.009537 & $0.016431-0.000243$ & $0.003014-$ & -0.003491 & & 0 & 1 \\
\hline $\mathrm{H} 6 \mathrm{~A}$ & -0.375000 & $1.000000 \quad 0.092339$ & 0.004576 & 0.685888 & 0.0 & & \\
\hline 0.019268 & 0.012340 & $0.014229-0.000125$ & $0.002520-$ & -0.004920 & & 0 & 1 \\
\hline $\mathrm{H} 6 \mathrm{~B}$ & -0.375000 & $1.0000000 .009600-$ & -0.018900 & 0.814691 & 0.0 & & \\
\hline 0.014224 & 0.009647 & $0.026857-0.003061$ & 0.006299 & 0.000168 & & 0 & 1 \\
\hline H7A & -0.375000 & $1.000000 \quad 0.316741$ & 0.107454 & 1.094270 & 0.0 & & \\
\hline 0.016598 & 0.016936 & $0.012393 \quad 0.003607$ & 0.001040 & 0.000321 & & 0 & 1 \\
\hline $\mathrm{H} 7 \mathrm{~B}$ & -0.375000 & $1.000000 \quad 0.138340$ & 0.092052 & 1.052878 & 0.0 & & \\
\hline 0.017074 & 0.014780 & $0.014417 \quad 0.001660$ & 0.007380 & 0.002011 & & 0 & 1 \\
\hline H9 & -0.375000 & $1.000000-0.177376$ & 0.080634 & 0.699229 & 0.0 & & \\
\hline 0.014017 & 0.019179 & $0.019187-0.004154$ & $0.001724=$ & $=0.005322$ & & 0 & 1 \\
\hline $\mathrm{H} 10$ & -0.375000 & $1.000000-0.389010$ & 0.146805 & 0.746257 & 0.0 & & \\
\hline 0.010867 & 0.027857 & $0.025083-0.003438$ & 0.003403 & 0.001062 & & 0 & 1 \\
\hline $\mathrm{H} 11$ & -0.375000 & $1.000000-0.387954$ & 0.322178 & 0.907661 & 0.0 & & \\
\hline 0.015401 & 0.023080 & $0.028789 \quad 0.005406$ & 0.011698 & 0.006558 & & 0 & 1 \\
\hline $\mathrm{H} 12$ & -0.375000 & $1.000000-0.172867$ & 0.427707 & 1.023741 & 0.0 & & \\
\hline 0.021232 & 0.019665 & $0.030399 \quad 0.002995$ & $0.013356-$ & -0.006345 & & 0 & 1 \\
\hline $\mathrm{H} 13$ & -0.375000 & $1.000000 \quad 0.036386$ & 0.362903 & 0.977271 & 0.0 & & \\
\hline 0.015325 & 0.017015 & $0.026705-0.002320$ & $0.007502-$ & -0.010296 & & 0 & 1 \\
\hline $\mathrm{HO} 2$ & -0.375000 & $1.000000 \quad 0.071033$ & 0.317748 & 0.672629 & 0.0 & & \\
\hline 0.013588 & 0.011864 & $0.013832-0.001003$ & 0.004119 & 0.003274 & & 0 & 1 \\
\hline
\end{tabular}




\begin{tabular}{|c|c|c|c|c|c|c|c|c|c|}
\hline \multicolumn{10}{|l|}{201} \\
\hline 301 & 7.2 & \multicolumn{3}{|c|}{5.4} & 12 & 1.0 & & & \\
\hline 401 & 155501 & \multicolumn{3}{|c|}{-2955501} & & & & & \\
\hline 501 & 555501 & \multicolumn{3}{|c|}{655501} & 255501 & 255501 & 355501 & & 0 \\
\hline 502 & \multirow[t]{2}{*}{2} & \multicolumn{3}{|c|}{28} & 1 & 2.7 & & & \\
\hline 604 & & & & & & 1.00 & & & \\
\hline 601 & 4.0 & \multicolumn{3}{|c|}{1.5} & & & & & \\
\hline \multirow{2}{*}{1001} & & & & & & & & & \\
\hline & 129 & 29 & 3 & .80 & 1.6 & .05 & & & \\
\hline \multirow{2}{*}{\multicolumn{10}{|c|}{$\begin{array}{l}716 \\
812\end{array}$}} \\
\hline & & & & & & & & & \\
\hline & $\begin{array}{lll}1 & 29 & 1\end{array}$ & 29 & 3 & .80 & 1.6 & .05 & & & \\
\hline 901 & 155501 & & & & & & .10 & -.05 & -.22 \\
\hline 901 & 255501 & & & & & & .10 & +.10 & -.22 \\
\hline 901 & 355501 & & & & & & .10 & +.17 & -.22 \\
\hline 901 & 455501 & & & & & & .10 & +.05 & -.212 \\
\hline 901 & 555501 & & & & & & .10 & -.20 & -.12 \\
\hline 901 & 655501 & & & & & & .10 & -.17 & -.22 \\
\hline 901 & 755501 & & & & & & .10 & -.25 & -.03 \\
\hline 901 & 855501 & & & & & & .10 & -.063 & -.22 \\
\hline 901 & 955501 & & & & & & .10 & -.27 & -.07 \\
\hline 901 & 1055501 & & & & & & .10 & +.05 & -.30 \\
\hline 901 & 1155501 & & & & & & .10 & -.25 & -.17 \\
\hline 901 & 1255501 & & & & & & .10 & +.27 & +.07 \\
\hline 901 & 1355501 & & & & & & .10 & -.12 & +.27 \\
\hline 901 & 1455501 & & & & & & .10 & +.02 & -.30 \\
\hline 901 & 1555501 & & & & & & .10 & +.22 & -.185 \\
\hline 901 & 1655501 & & & & & & .10 & 0.00 & +.27 \\
\hline 901 & 1755501 & & & & & & .10 & +.10 & -.27 \\
\hline 901 & 1855501 & & & & & & .10 & +.35 & +.05 \\
\hline 901 & 1955501 & & & & & & .10 & 0.00 & +.27 \\
\hline 901 & 2055501 & & & & & & .10 & +.05 & -.265 \\
\hline 901 & 2155501 & & & & & & .10 & -.40 & +.10 \\
\hline 901 & 2255501 & & & & & & .10 & +.35 & 0.00 \\
\hline 901 & 2355501 & & & & & & .10 & -.40 & 0.00 \\
\hline 901 & 2455501 & & & & & & .10 & -.33 & -.07 \\
\hline 901 & 2555501 & & & & & & .10 & 0.00 & -.33 \\
\hline 901 & 2655501 & & & & & & .10 & -.05 & -.30 \\
\hline 901 & 2755501 & & & & & & .10 & +.30 & +.12 \\
\hline 901 & 2855501 & & & & & & .10 & +.012 & +.32 \\
\hline 901 & 2955501 & & & & & & .10 & -.475 & 0.00 \\
\hline 202 & & & & & & & & & \\
\hline
\end{tabular}

This second example shows the packing of the norbornanone molecules in a unit cell. The unit cell contains four molecules, but six have been drawn. The hydrogen atoms have been omitted for clarity, and the carbon and oxygen atoms have been drawn with different representations for easier visual identification. (The oxygen atoms are the ellipsoids with the shaded octant.) The input shows the new format for the symmetry operators that is available in ORTEP-III. In the atom parameters, dummy atoms have been provided for a corner of the unit cell (atom \#16 at $0 ., 0 ., 0$.) and one for its center (atom \#17 at $.5, .5, .5$ ). 

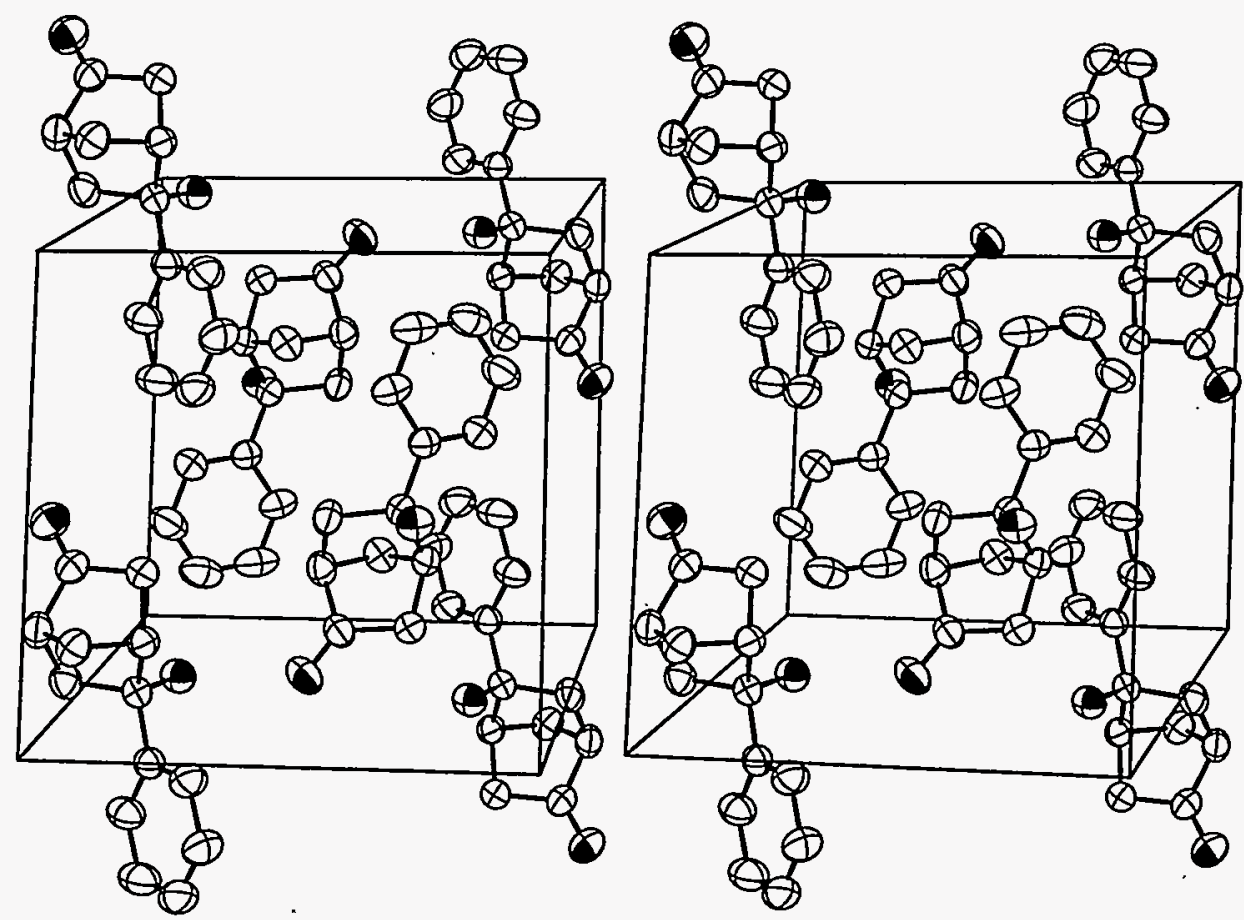

Fig. 7.2. Packing diagram of 5-hydroxy-5-phenylnorbornanone.

PHENYL HYDROXYL NORBORNANONE

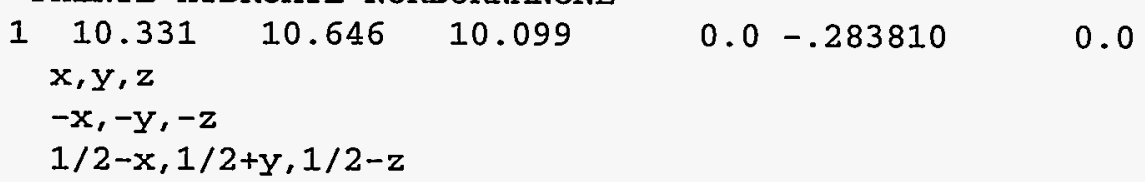

$11 / 2+x, 1 / 2-y, 1 / 2+z$

$\begin{array}{llllllll}\text { C1 } & 0.661000 & 1.000000 & 0.224802 & 0.001638 & 0.901515 & 0.0\end{array}$

$\begin{array}{llllllll}0.011930 & 0.006750 & 0.011647 & 0.000642 & 0.003989 & 0.000860\end{array}$

$\begin{array}{lllllllll}\text { C2 } & 0.661000 & 1.000000 & 0.335043 & 0.050840 & 0.844329 & 0.0\end{array}$

$\begin{array}{lllllll}0.010432 & 0.007831 & 0.012112 & 0.001274 & 0.003947-0.000264\end{array}$

$\begin{array}{lllllllll}\text { C3 } & 0.661000 & 1.000000 & 0.327553 & 0.192798 & 0.850375 & 0.0\end{array}$

$\begin{array}{lllllll}0.009129 & 0.007883 & 0.013469-0.000262 & 0.003784 & 0.000058\end{array}$

$\begin{array}{lllllllll}\mathrm{C} 4 & 0.661000 & 1.000000 & 0.206609 & 0.213473 & 0.908171 & 0.0\end{array}$

$\begin{array}{llllll}0.008568 & 0.006819 & 0.009641-0.000310 & 0.002118-0.001032\end{array}$

$\begin{array}{llllllll}\text { C5 } & 0.661000 & 1.000000 & 0.074610 & 0.177193 & 0.796977 & 0.0\end{array}$

$\begin{array}{lllllll}0.008590 & 0.006732 & 0.008260-0.000670 & 0.002360 & 0.000103\end{array}$

$\begin{array}{lllllllll}\text { C6 } & 0.661000 & 1.000000 & 0.091094 & 0.031659 & 0.790120 & 0.0\end{array}$

$0.011368 \quad 0.006963 \quad 0.012116-0.001403 \quad 0.003143-0.001297$

C7 $\quad 0.661000 \quad 1.000000 \quad 0.2236790 .102901 \quad 1.010406 \quad 0.0$

$\begin{array}{llllllll}0.011840 & 0.009377 & 0.008981 & 0.001423 & 0.002835 & 0.000648\end{array}$

$\begin{array}{llllllllll}\text { C8 } & 0.661000 & 1.000000-0.053735 & 0.216149 & 0.834431 & 0.0\end{array}$

$\begin{array}{lllllll}0.008328 & 0.007955 & 0.008995-0.000100 & 0.002374 & 0.001216\end{array}$

$\begin{array}{llllllll}\text { C9 } & 0.661000 & 1.000000-0.175896 & 0.157400 & 0.769638 & 0.0\end{array}$

$\begin{array}{llllllll}0.008481 & 0.012396 & 0.011267-0.001406 & 0.001677 & 0.001394\end{array}$

$\begin{array}{lllllll}\mathrm{C} 10 & 0.661000 & 1.000000-0.296122 & 0.195813 & 0.795042 & 0.0\end{array}$

$\begin{array}{lllllll}0.008526 & 0.016481 & 0.014906-0.000226 & 0.002743 & 0.004954\end{array}$

$\begin{array}{lllllllll}\text { C11 } & 0.661000 & 1.000000-0.295600 & 0.294171 & 0.886394 & 0.0\end{array}$

$$
\begin{array}{lll}
0 & 1 & 0 \\
0 & 1 & 0 \\
0 & 1 & 0 \\
0 & 1 & 0 \\
0 & 1 & 0 \\
0 & 1 & 0 \\
0 & 1 & 0 \\
0 & 1 & 0 \\
0 & 1 & 0 \\
0 & 1 & 0
\end{array}
$$




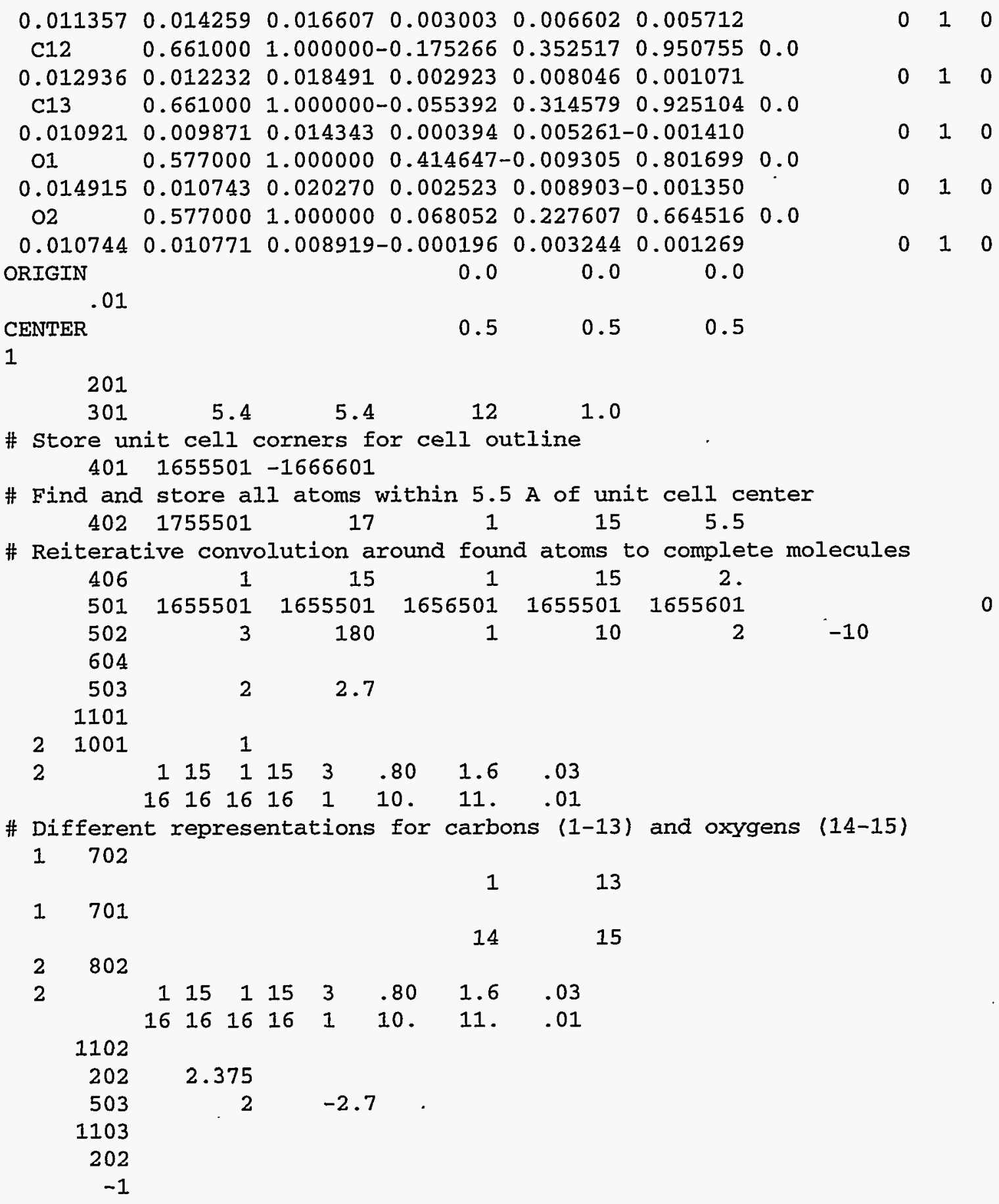

\subsection{HELICAL STRUCTURE - POLY-L-ALANINE}

The structure of poly-L-alanine was published by Elliott and Malcolm in $1959 .^{25}$ The Pauling and Corey right-handed alpha helix repeats after 13 turns and 47 residues and can be represented in ORTEP by 47 symmetry cards with $N=47 ; M=13 ; L=0,1, \ldots, 46 ; T_{1}, T_{2}, T_{3}=0$. The input atom list then contains the contents of one residue. In this example there are 48 symmetry cards with operator 1 and operator 48 related by one cell translation along $c$. 

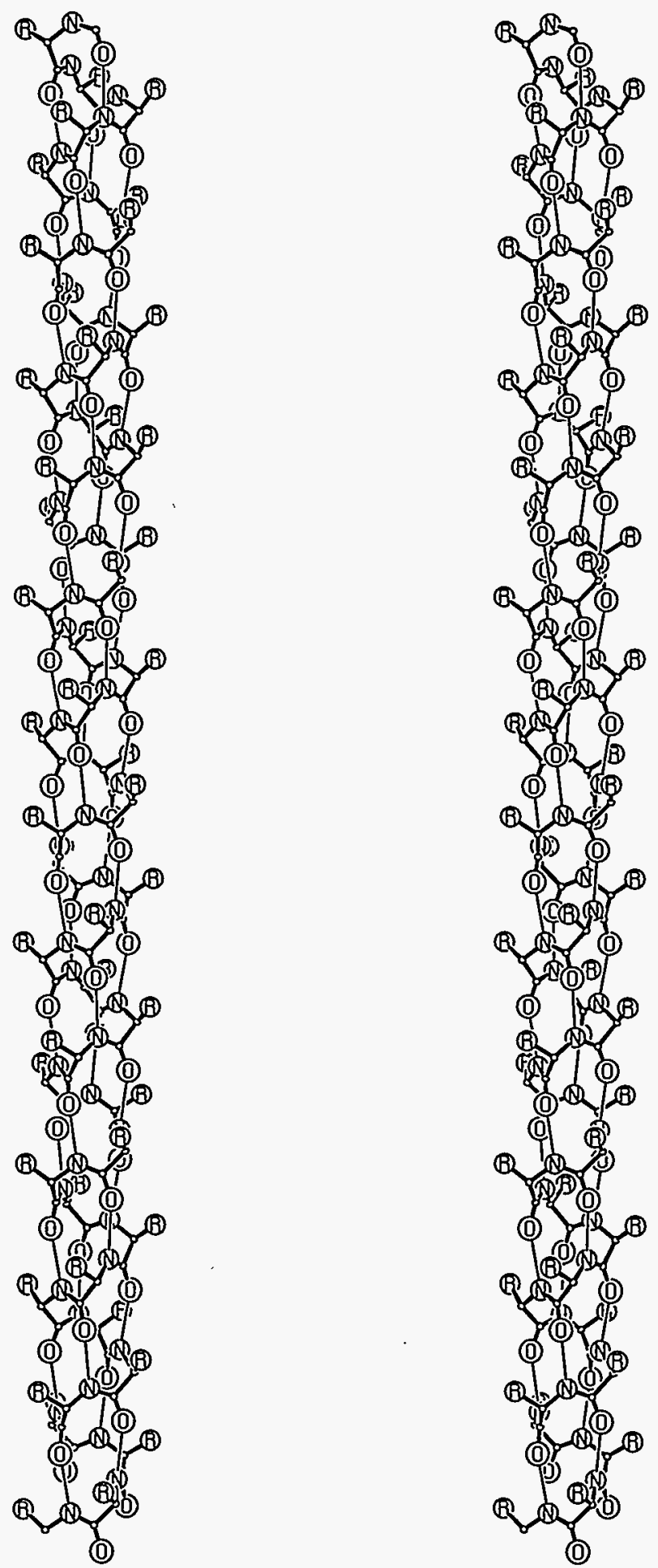

Fig. 7.3. $47 / 13 \alpha$-Helix of poly-L-alanine. The thin "vertical" lines between nitrogen and oxygen atoms indicate a hydrogen bond path. 
POLY-I-ALANINE $47 / 13$ HELIX

8.55

8.55

70.3
ELLIOTT AND MALCOLM (1959)

90 .

90 .

120. $\begin{array}{lll}0 & 13 \quad 47\end{array}$

$\begin{array}{lll}1 & 13 & 47\end{array}$

$\begin{array}{llll}2 & 13 & 47\end{array}$

$\begin{array}{llll}3 & 13 & 47\end{array}$

$\begin{array}{llll}4 & 13 & 47\end{array}$

$\begin{array}{llll}5 & 13 & 47\end{array}$

$\begin{array}{llll}6 & 13 & 47\end{array}$

$\begin{array}{llll}7 & 13 & 47\end{array}$

$\begin{array}{lll}8 & 13 & 47\end{array}$

$\begin{array}{lll}9 & 13 & 47\end{array}$

$\begin{array}{lll}10 & 13 & 47\end{array}$

$\begin{array}{lll}11 & 13 & 47\end{array}$

$\begin{array}{lll}12 & 13 & 47\end{array}$

$\begin{array}{lll}13 & 13 & 47\end{array}$

$\begin{array}{lll}14 & 13 & 47\end{array}$

$\begin{array}{lll}15 & 13 & 47\end{array}$

$\begin{array}{lll}16 & 13 & 47\end{array}$

.17 $13 \quad 47$

$\begin{array}{lll}18 & 13 & 47\end{array}$

$\begin{array}{lll}19 & 13 & 47\end{array}$

$\begin{array}{lll}20 & 13 & 47\end{array}$

$\begin{array}{lll}21 & 13 & 47\end{array}$

$\begin{array}{lll}22 & 13 & 47\end{array}$

$\begin{array}{lll}23 & 13 & 47\end{array}$

$\begin{array}{lll}24 & 13 & 47\end{array}$

$\begin{array}{lll}25 & 13 & 47\end{array}$

$\begin{array}{lll}26 & 13 & 47\end{array}$

$\begin{array}{lll}27 & 13 & 47\end{array}$

$\begin{array}{lll}28 & 13 & 47\end{array}$

$\begin{array}{lll}29 & 13 & 47\end{array}$

$\begin{array}{lll}30 & 13 & 47\end{array}$

$\begin{array}{lll}31 & 13 & 47\end{array}$

$\begin{array}{lll}32 & 13 & 47\end{array}$

$\begin{array}{lll}33 & 13 & 47\end{array}$

$\begin{array}{lll}34 & 13 & 47\end{array}$

$\begin{array}{lll}35 & 13 & 47\end{array}$

$\begin{array}{lll}36 & 13 & 47\end{array}$

$\begin{array}{lll}37 & 13 & 47\end{array}$

$\begin{array}{lll}38 & 13 & 47\end{array}$

$\begin{array}{lll}39 & 13 & 47\end{array}$

$\begin{array}{lll}40 & 13 & 47\end{array}$

$\begin{array}{lll}41 & 13 & 47\end{array}$

$\begin{array}{lll}42 & 13 & 47\end{array}$

$\begin{array}{lll}43 & 13 & 47\end{array}$

$\begin{array}{lll}44 & 13 & 47\end{array}$

$\begin{array}{lll}45 & 13 & 47\end{array}$

$\begin{array}{lll}46 & 13 & 47\end{array}$

$47 \quad 13 \quad 47$

1

$\begin{array}{lll}1.63 & 94.9 & -.40\end{array}$

3
7 


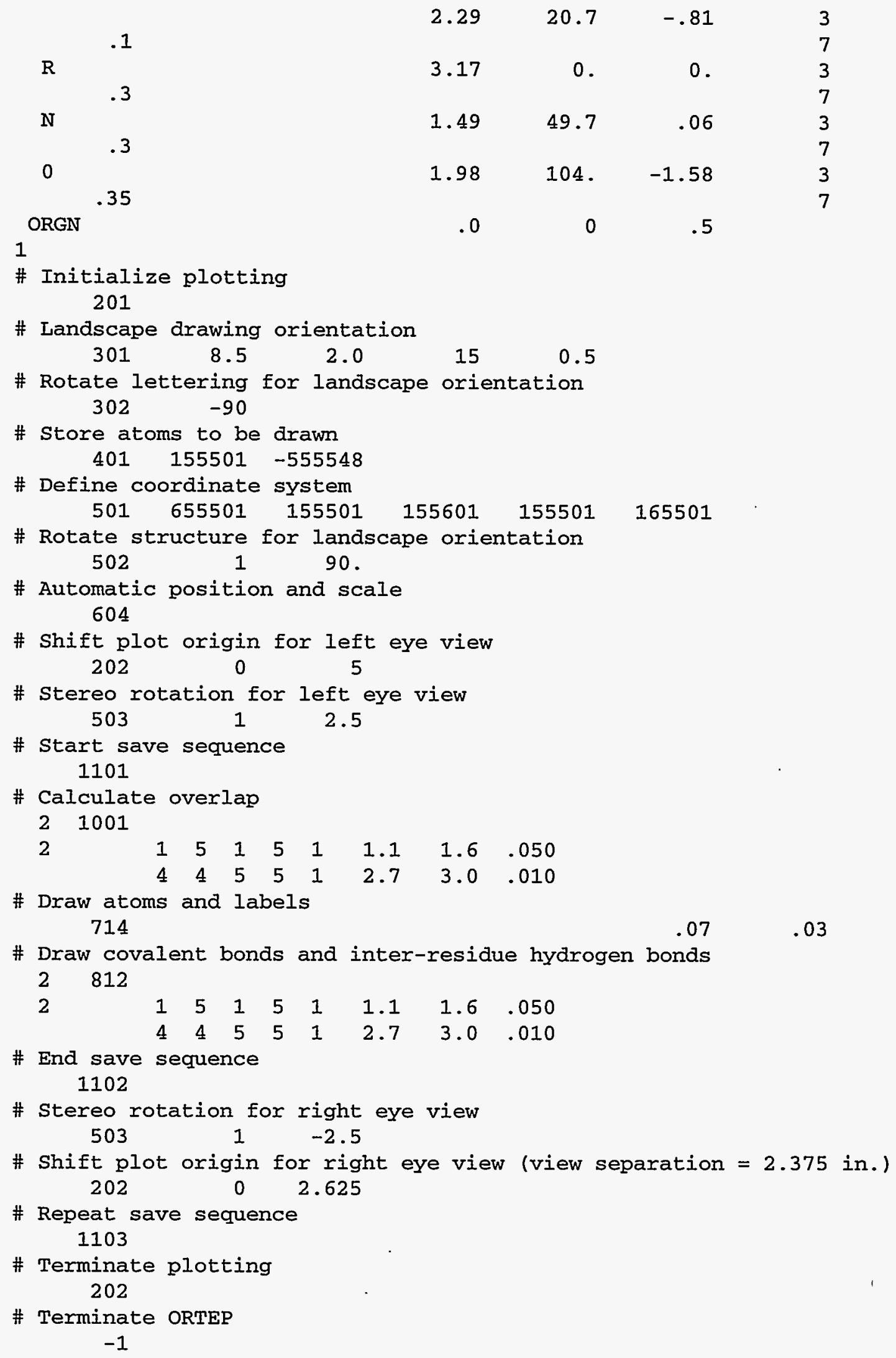




\subsection{COORDINATION POLYHEDRA - POTASSIUM PERXENATE NONAHYDRATE}

The crystal structure of this hydrated ionic material was published by Zalkin et al. in 1964. ${ }^{26}$ The only covalent bonds are between the xenon and oxygen atoms in the perxenate anions (the darker bonds in Fig. 7.4). To see better how the oxygens of the perxenate anions and water molecules coordinate around the potassium and atoms, lines have been drawn from the potassiums to all oxygens within a distance of $3.3 \AA$.
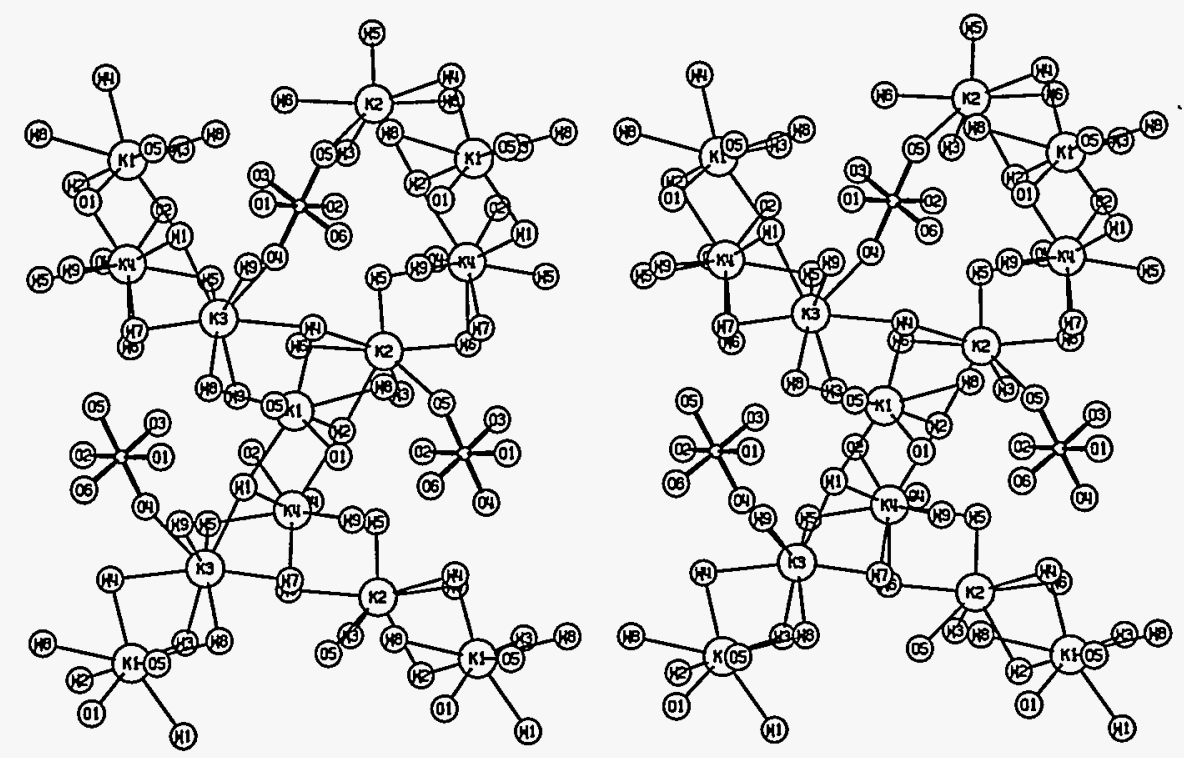

Fig. 7.4. Coordination polyhedra in potassium perxenate nonahydrate.

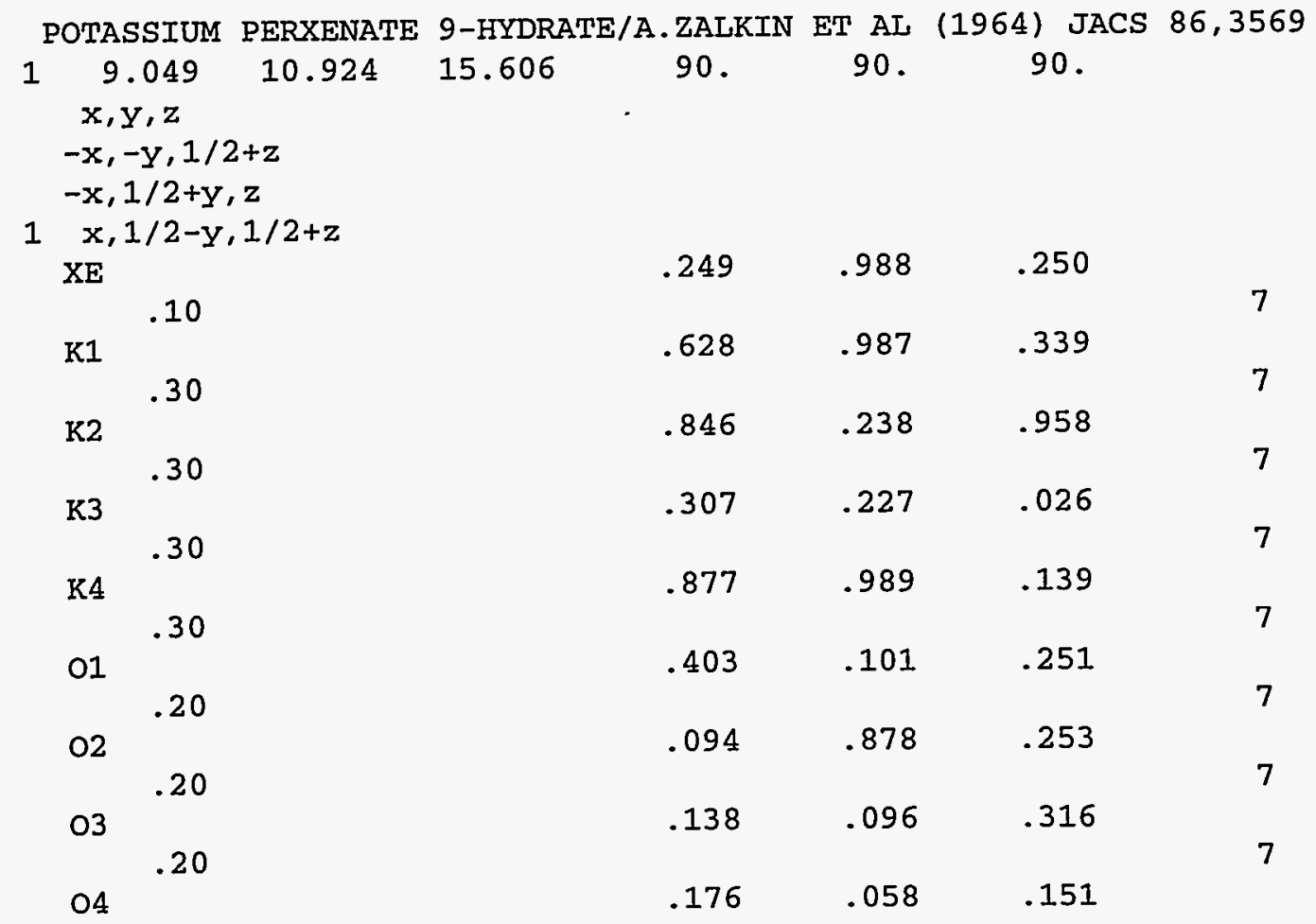




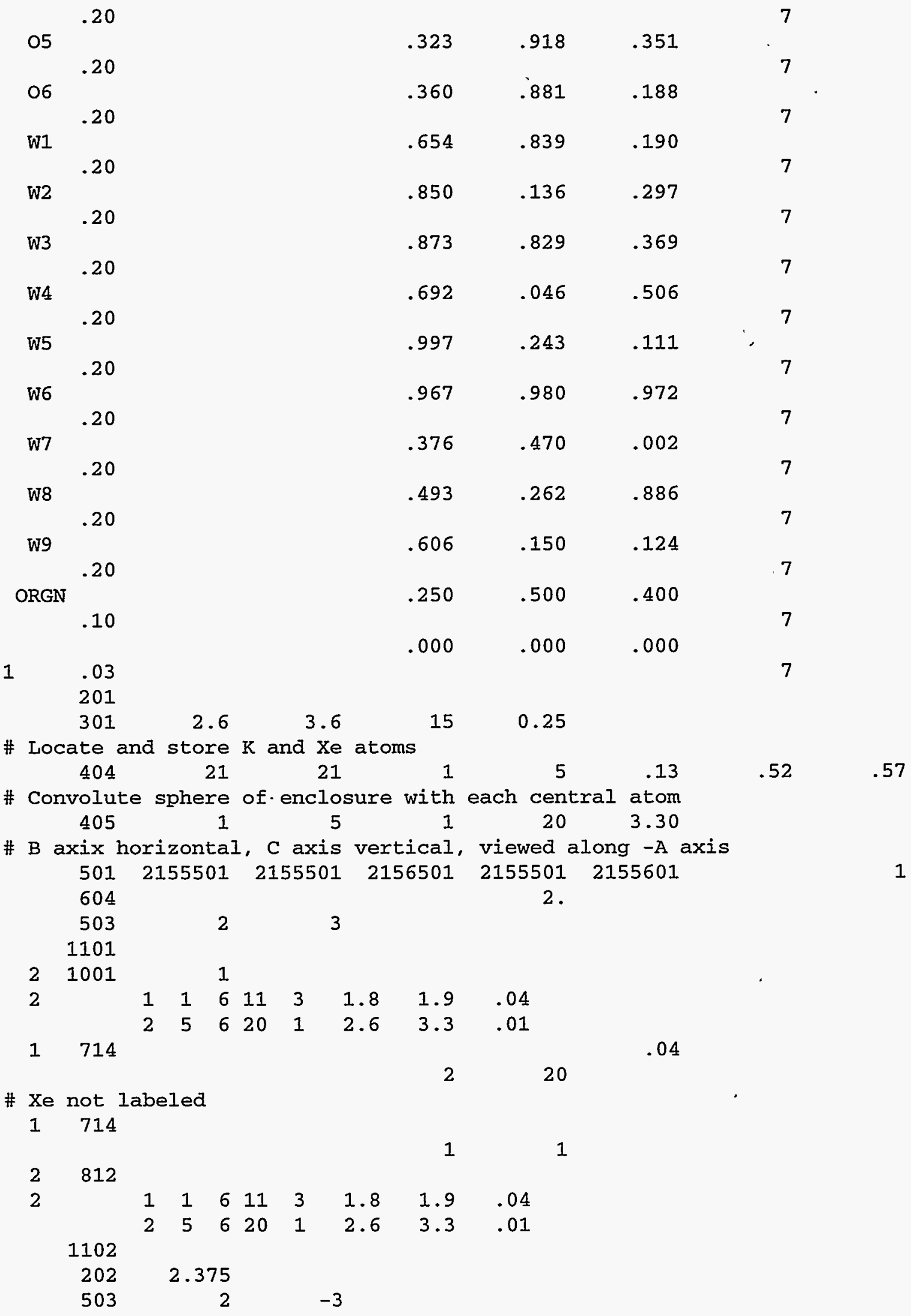


In the following representation of the same structure shown in Fig. 7.4, only the xenon and potassium atoms have been explicitly drawn. (The xenon atoms are the smaller circles.) The oxygen atoms are shown implicitly as the vertices of polyhedra centered on the potassium and xenon atoms. As in the previous case, oxygens within $3.3 \AA$ of the potassium are treated as making up the coordination polyhedron. Only the instruction portion of the input file is provided. The input lines that precede these are the same as those in the previous case.
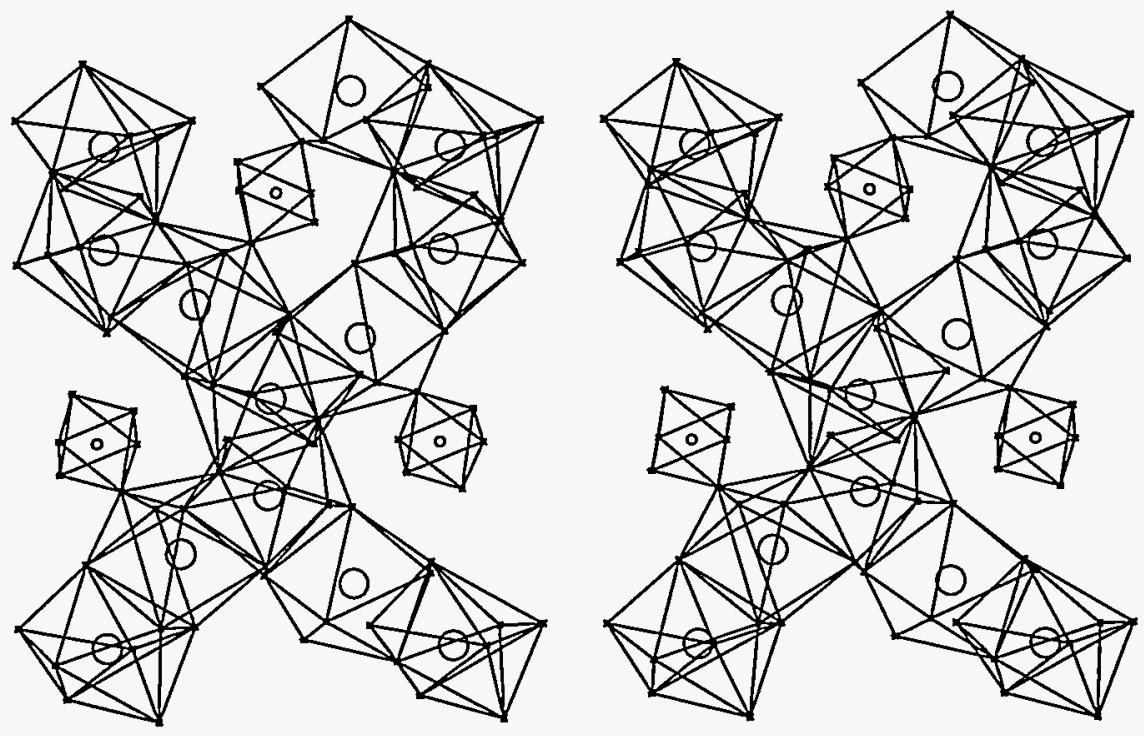

Fig. 7.5. Coordination polyhedra in potassium perxenate nonahydrate.

201

$\begin{array}{lllll}301 & 2.6 & 3.6 & 15 & 0.25\end{array}$

\# Locate and store $K$ and $X e$ atoms

$\begin{array}{llllll}404 & 21 & 21 & 1 & 5 & .13\end{array}$

\# Convolute sphere of enclosure with each central atom
405
1
5
120
3.30

\# B axix horizontal, C axis vertical, viewed along $-A$ axis

$\begin{array}{llllll}501 & 2155501 & 2155501 & 2156501 & 2155501 & 2155601\end{array}$

604

503

2

3

1101

\# Draw xenon and potassium atoms only

1714

1

5

\# Use polygon radii to limit bonds drawn 2813

2

$$
\begin{array}{llll}
6 & 11 & 6 & 11
\end{array}
$$

$620 \quad 6 \quad 20$

$\begin{array}{ll}2.5 & 2.8 \\ 2.6 & 4.6\end{array}$

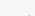




\subsection{ATOM FEATURES - LYSOSOME MUTANT POLYPEPTIEE}

The data for this example were taken from the Protein Data Bank \#216L. The header information from that file is provided below.

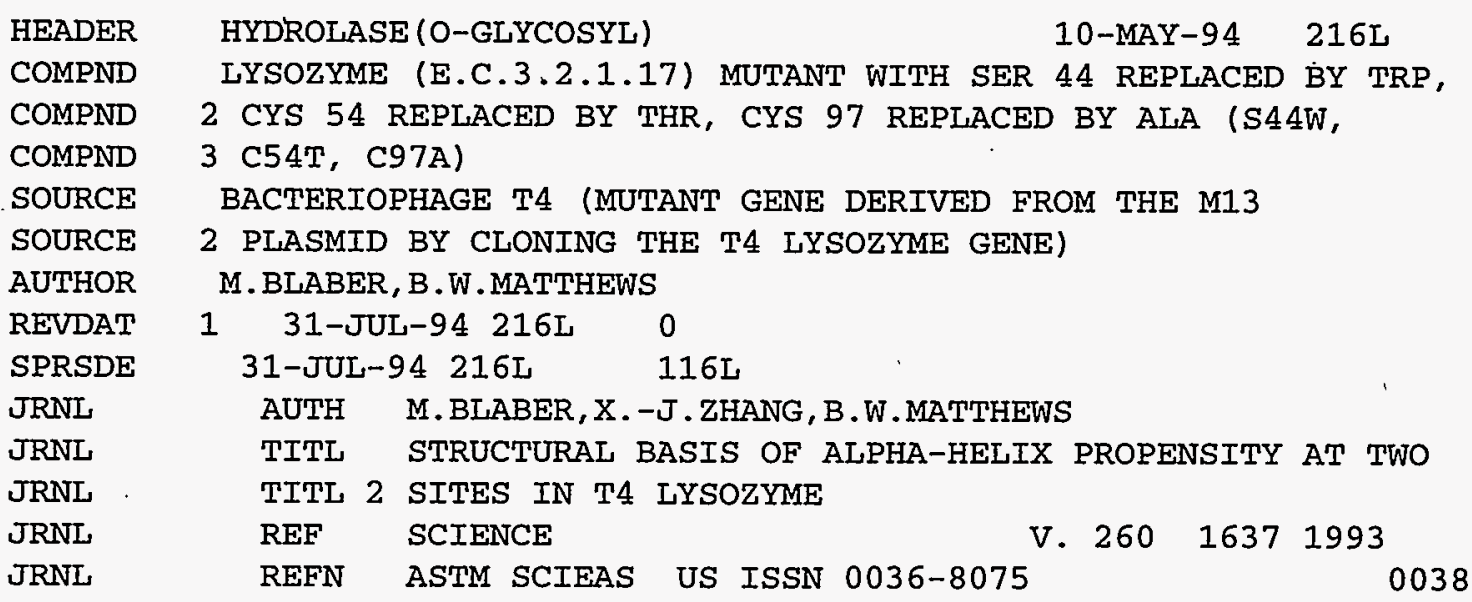

Only the first 63 amino acids (500 atoms) of the protein were used for this example since that is the size used in the dimension statements in ORTEP-III. The first 500 ATOM lines were extracted from the PDB file and placed unaltered in a file named ATOMS.DAT. A few of the lines are shown below.

\begin{tabular}{|c|c|c|c|c|c|c|c|c|c|c|c|}
\hline ATOM & 1 & $\mathrm{~N}$ & MET A & 1 & 82.486 & 23.405 & 25.378 & 1.00 & 29.06 & 216L & 127 \\
\hline ATOM & 2 & $C A$ & MET A & 1 & 81.291 & 22.758 & 24.885 & 1.00 & 15.78 & $216 \mathrm{~L}$ & 128 \\
\hline ATOM & 3 & C & MET A & 1 & 80.495 & 23.789 & 24.150 & 1.00 & 33.32 & $216 \mathrm{~L}$ & 129 \\
\hline ATOM & 4 & 0 & MET A & 1 & 80.951 & 24.925 & 24.017 & 1.00 & 29.09 & $216 \mathrm{~L}$ & 130 \\
\hline ATOM & 5 & $\mathrm{CB}$ & MET A & 1 & 80.556 & 22.168 & 26.090 & 1.00 & 14.87 & $216 \mathrm{~L}$ & 131 \\
\hline ATOM & 6 & CG & MET A & 1 & 79.353 & 21.283 & 25.811 & 1.00 & 44.92 & $216 L$ & 132 \\
\hline ATOM & 7 & SD & MET A & 1 & 78.906 & 20.301 & 27.306 & 1.00 & 34.12 & $216 L$ & 133 \\
\hline ATOM & 8 & $\mathrm{CE}$ & MET A & 1 & 80.536 & 19.686 & 27.844 & 1.00 & 7.96 & $216 \mathrm{~L}$ & 134 \\
\hline ATOM & 9 & $\mathbf{N}$ & ASN A & 2 & 79.348 & 23.416 & 23.650 & 1.00 & 7.39 & $216 \mathrm{~L}$ & 135 \\
\hline ATOM & 10 & $\mathrm{CA}$ & ASN A & 2 & 78.619 & 24.379 & 22.897 & 1.00 & 14.21 & $216 \mathrm{~L}$ & 136 \\
\hline$\cdot$ & & & $\cdot$ & & • & & - & & - & & - \\
\hline • & & & • & & - & & • & & • & & - \\
\hline$\dot{x}$ & & & 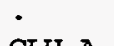 & & ¿0 & & 7 roم & & $\dot{i}$ & & $\dot{i n}$ \\
\hline ATOM & 491 & $\mathrm{CB}$ & GLU A & 62 & 69.880 & 12.430 & 7.589 & 1.00 & 6.64 & $216 \mathrm{~L}$ & 617 \\
\hline ATOM & 492 & $C G$ & GLU A & 62 & 70.251 & 11.994 & 6.135 & 1.00 & 1.34 & $216 \mathrm{~L}$ & 618 \\
\hline ATOM & 493 & $\mathrm{CD}$. & GLU A & 62 & 69.487 & 10.795 & 5.671 & 1.00 & 27.84 & $216 \mathrm{~L}$ & 619 \\
\hline ATOM & 494 & OE1 & GLU A & 62 & 68.805 & 10.091 & 6.416 & 1.00. & 19.47 & $216 L$ & 620 \\
\hline ATOM & 495 & $\mathrm{OE} 2$ & GLU A & 62 & 69.547 & 10.652 & 4.368 & $1.00^{\circ}$ & 34.69 & $216 \mathrm{~L}$ & 621 \\
\hline ATOM & 496 & $\mathrm{~N}$ & $A L A A$ & 63 & 70.531 & 13.275 & 10.600 & 1.00 & 32.33 & $216 \mathrm{~L}$ & 622 \\
\hline ATOM & 497 & $\mathrm{CA}$ & ATAA A & 63 & 70.126 & 13.774 & 11.873 & 1.00 & 6.04 & $216 L$ & 623 \\
\hline ATOM & 498 & $c$ & $A I A A$ & 63 & 70.877 & 15.054 & 12.241 & 1.00 & 55.04 & $216 \mathrm{I}$ & 624 \\
\hline ATOM & 499 & 0 & ALA A & 63 & 70.278 & 16.027 & 12.662 & 1.00 & 13.24 & 216I & 625 \\
\hline ATOM & 500 & $\mathrm{CB}$ & $A L A A$ & 63 & 70.323 & 12.701 & 12.964 & 1.00 & 18.46 & $216 \mathrm{~L}$ & 626 \\
\hline
\end{tabular}


Since these atom data are not in the standard format used by ORTEP, subroutine READIN was written to read this particular format. It is shown below. As each atom is read by READIN, the subroutine sets the value of FEATURE \#2 (id2) for the atom to the sequence number of the amino acid containing the atom. FEATURE \#1 (id1) is set to a value that indicates the type of atom:

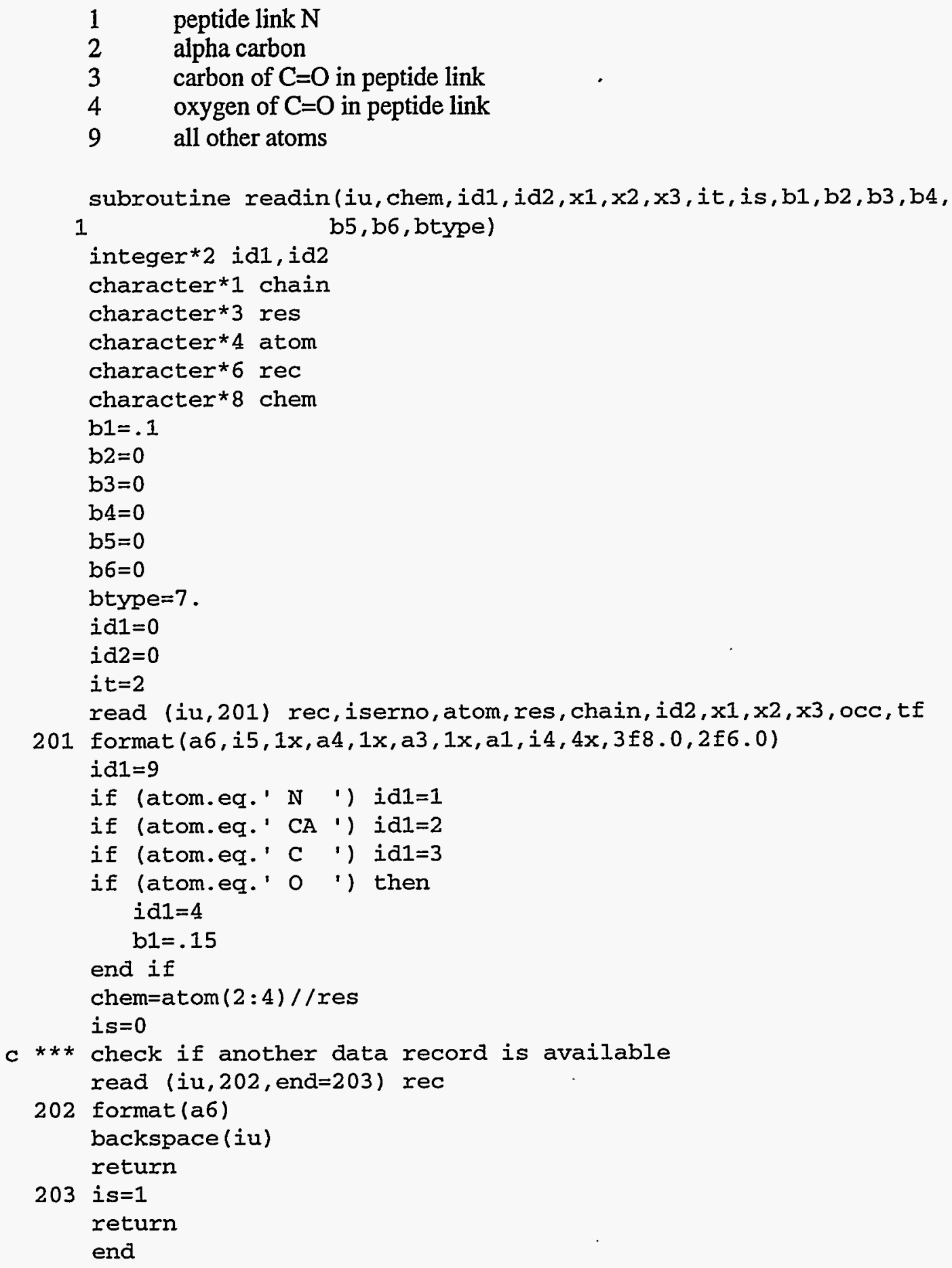


The ORTEP input file contains the instructions for producing three different illustrations. Each begins with a 201 instruction and ends with a 202. The second and third sets make use of the assigned atom features to select particular atoms for drawing. In the input file, a " 2 " in column 1 of the final symmetry card tells ORTEP (1) that the atom data are in a separate file and (2) to use subroutine READIN to read the data.
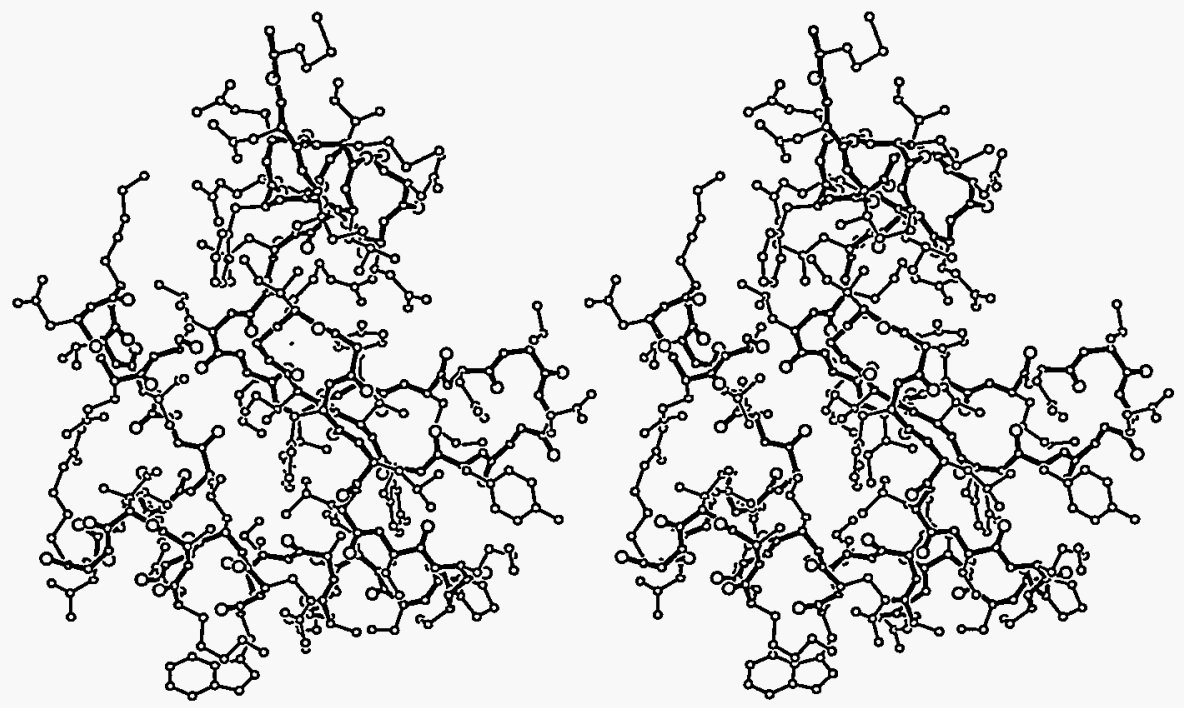

Fig. 7.6. First 63 amino acids of lysosome mutant protein.
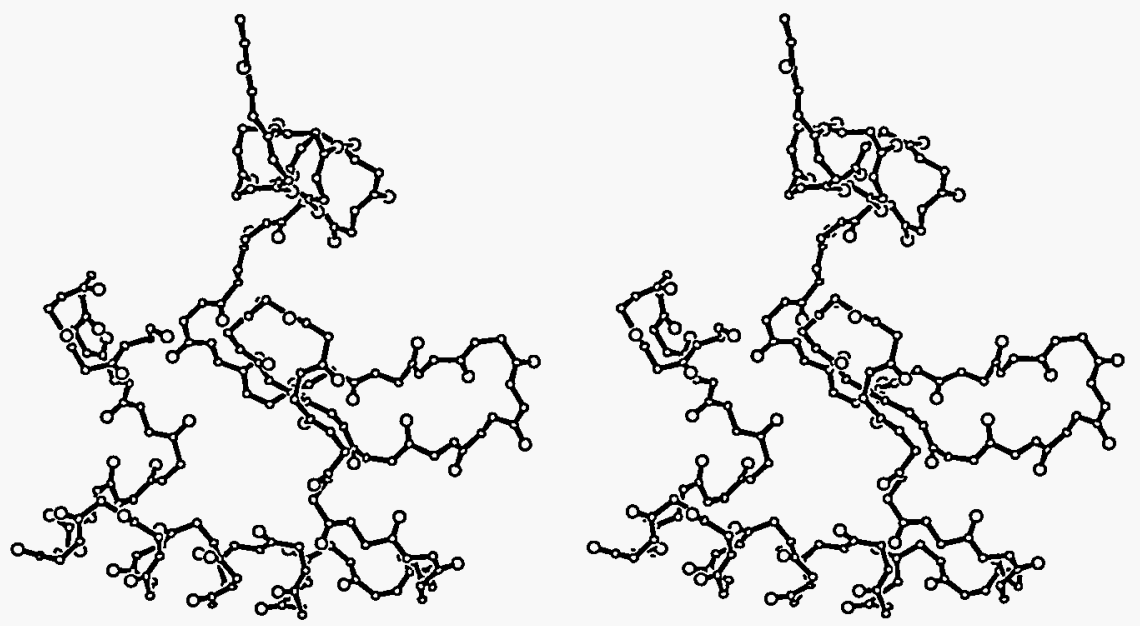

Fig. 7.7. First 63 amino acids of lysosome mutant protein with side chains eliminated. 

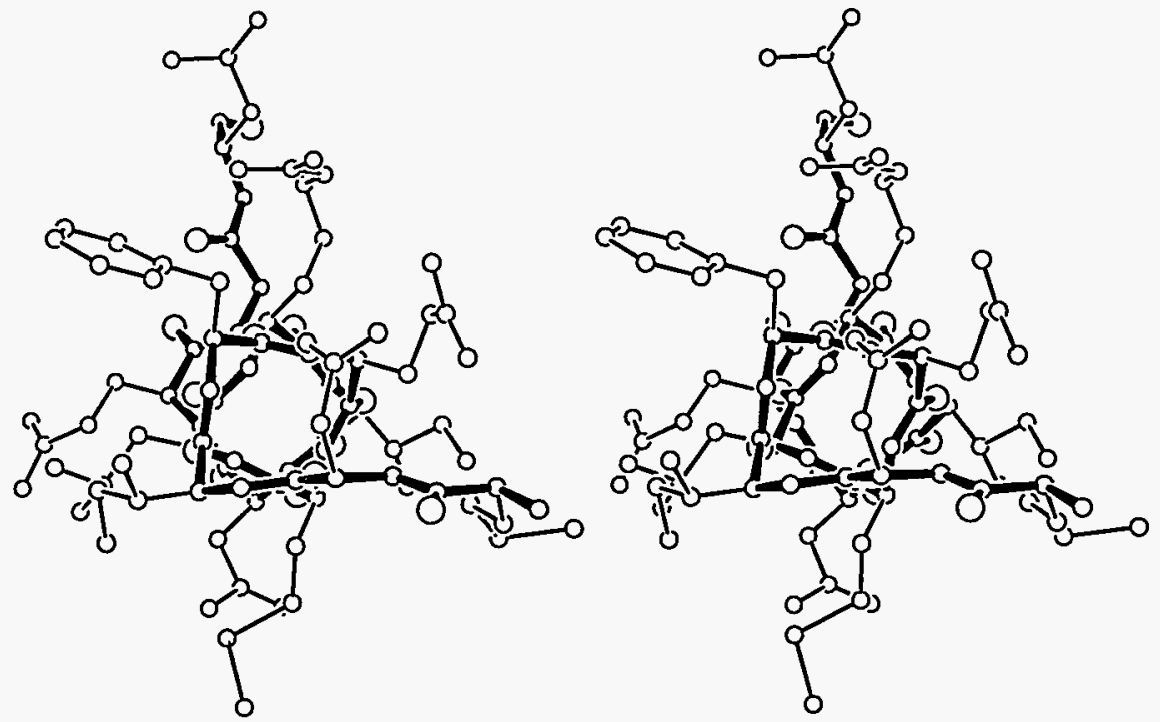

Fig. 7.8. First 13 amino acids of lysosome mutant protein, looking through $\alpha$-helix.

IYSOZYME MUTANT PROTEIN DATA BANK \#216L - BLABER AND MATTHEWS
1116.500
54.400
59.500
90.00
102.30
90.00
$\mathrm{X}, \mathrm{Y}, \mathrm{Z}$
$-X, Y,-Z$
$\mathrm{X}+1 / 2, \mathrm{Y}+1 / 2, \mathrm{Z}$
$2-\mathrm{X}+1 / 2, \mathrm{Y}+1 / 2,-\mathrm{Z}$
\# Polypeptide containing first 63 amino acids of protein.
201
301
401
506
502
604
503
1101
21001
2
$1 \quad 714$
$\begin{array}{rr}5.0 & 3.5 \\ 155501-50055501\end{array}$
3
90
2
2.7

$\begin{array}{rrrrrrrr}1 & 4 & 1 & 4 & 5 & 0.9 & 2.0 & .08 \\ 1 & 4 & 5 & 9 & 1 & 0.9 & 2.0 & .02 \\ 5 & 9 & 5 & 9 & 1 & 0.9 & 2.0 & .02\end{array}$
1714
$\begin{array}{ll}2 & 812 \\ 2 & \\ 2 & \end{array}$
1102
202
503
1103
202
\# 63 amino acid polypeptide with side chains eliminated. 
\# The origin, axes, and scale are unchanged from above.

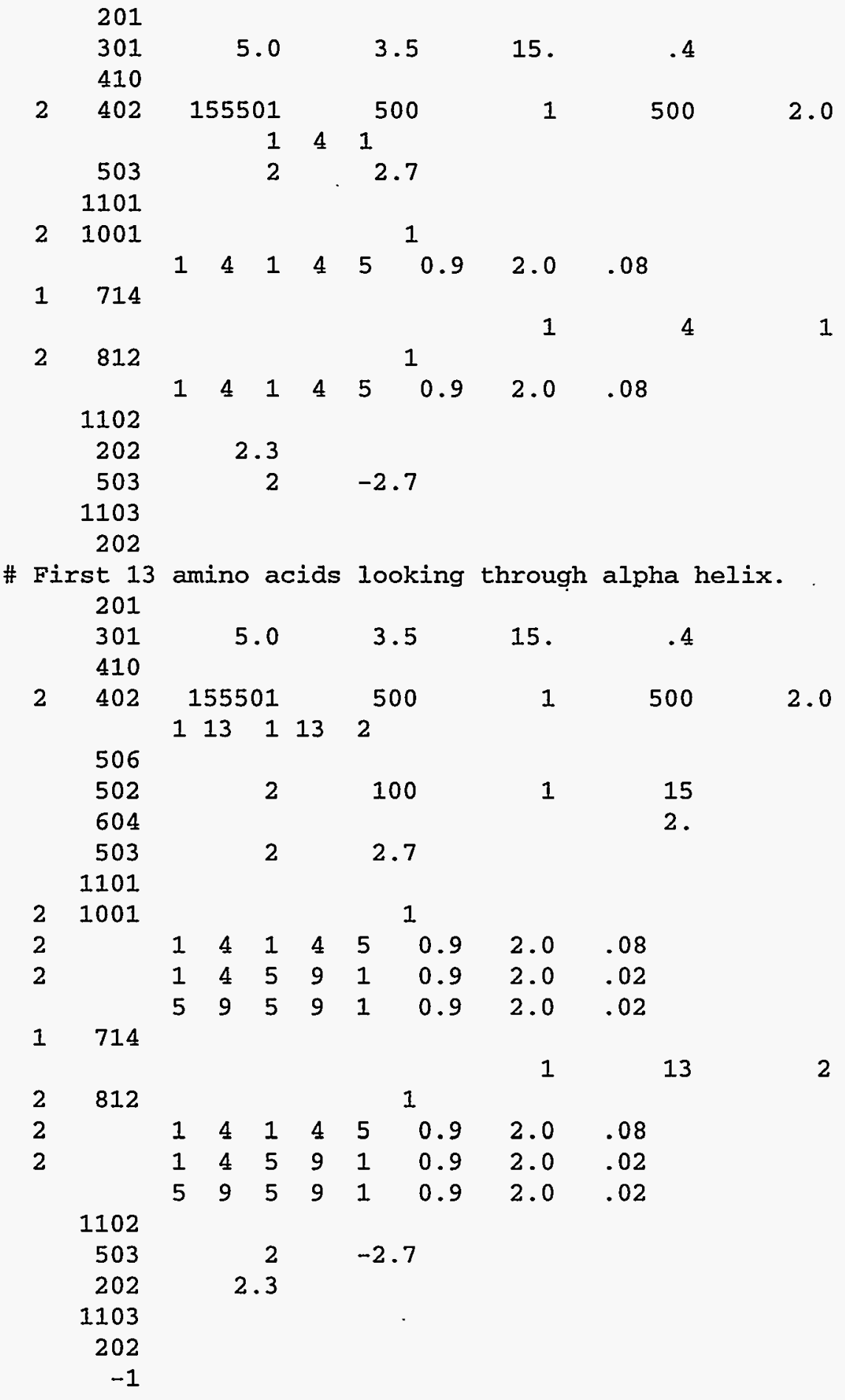

\subsection{CRITICAL NET - SODIUM CHLORDE}

ORTEP-III can produce "critical net" illustrations that depict some canonical topological characteristics of the global ensemble of overlapping atomic-thermal-motion Gaussian density 
functions in a crystal. Non-degenerate critical points occur where the first derivative of the global density is zero and the second derivative is a $3 \times 3$ symmetric matrix with a non-zero determinant. The signs of the three eigenvalues of the second derivative matrix specify the types of critical points, which are termed peak $(-,-,-)$, pass $(+,-,-)$, pale $(+,+,-)$ and pit $(+,+,+)$. Peaks correspond to density maxima, pits to density minima, and passes and pales to saddle points in the density function. The four types of critical points represent 0 (e.g., vertex), 1 (e.g., edge), 2 (e.g., face), and 3 (e.g., body) dimensional cells in the topological Morse function CW complex (i.e., $\mathrm{C}$ for closure finite, $\mathrm{W}$ for weak topology), simply called a critical net, and correspond with the number of + signs in the sign signature for each critical point. The most gradual up-density path from a pit to a peak follows the sequence pit $\rightarrow$ pale $\rightarrow$ pass $\rightarrow$ peak. A discussion of critical nets can be found on the World Wide Web at http://www.ornl.gov/ortep/topology/critnet.html.

Fig. 7.9 illustrates the critical net for $\mathrm{NaCl}$ with the larger corner spheres representing $\mathrm{Cl}$ peaks; the smaller corner spheres, Na peaks; the elongated "cigar-shaped" ellipsoids, passes; the flattened "pancake-shaped" ellipsoids, pales; and the smallest sphere in the center, a pit. The paths connecting the critical points, shown by the connection "bonds" in Fig. 7,9, are topologically unique.

New in ORTEP-III is the method for specifying the orientations and sizes of the elongated and flattened ellipsoids without giving their quadratic form coefficients. The temperature factor card following the atom parameter card for a pass or pale has the format:

\begin{tabular}{cc} 
Columns & \\
\hline 1 & A sentinel $\neq 0$ if last atom \\
$2-9$ & Unique axis length $(\AA)$ \\
$10-18$ & Second (and third) axis length $(\AA)$ \\
$19-27$ & $\mathrm{VDC}_{1}$ (from) \\
$28-36$ & $\mathrm{VDC}_{1}$ (to) \\
$37-45$ & {$\left[\mathrm{VDC}_{2}\right.$ (from) } \\
$46-54$ & $\mathrm{VDC}_{2}$ (to)] \\
$55-63$ & 7
\end{tabular}

$\mathrm{VDC}_{1}$ is a vector parallel with the unique axis of the cigar-shaped pass or pancake-shaped pale and $\mathrm{VDC}_{2}$ is a second vector not parallel with $\mathrm{VDC}_{1}$ such that $\mathrm{VDC}_{1} \times \mathrm{VDC}_{2}$ is a second principal axis of that ellipsoid. If $\mathrm{VDC}_{1}$ and $\mathrm{VDC}_{2}$ are parallel, $\mathrm{VDC}_{2}$ is replaced by a suitable lattice translation vector. $\mathrm{VDC}_{2}$ may be omitted from the input if desired, and the program will choose one of the three lattice vectors for $\mathrm{VDC}_{2}$.

This example illustrates an important point about the relationship between the symmetry operators and atom input data unrelated to the fact that this is a critical net drawing. Sodium chloride crystallizes in space group $\mathrm{Fm} \overline{3} \mathrm{~m}$, which has 192 symmetry operators. Of these, 48 are "unique", and the others may be obtained from these by adding the centering translations. The centering translations in this space group are $(0,0,0),(0, .5, .5),(.5,0, .5)$, and $(.5, .5,0)$. As discussed earlier (see 3.2.3), if all the symmetry operators are not provided in the ORTEP input file, each unique atom will require multiple entries with those centering translations added that are not provided in the symmetry cards. In this case only 48 symmetry operators have been included 
(although ORTEP-III allows a maximum of 96). As a consequence, each atom has four entries, obtained by adding the centering translation values to the atom's positional coordinates. If 96 operators had been included, each atom would have required two entries. The symmetry operators are provided in ORTEP's original format.

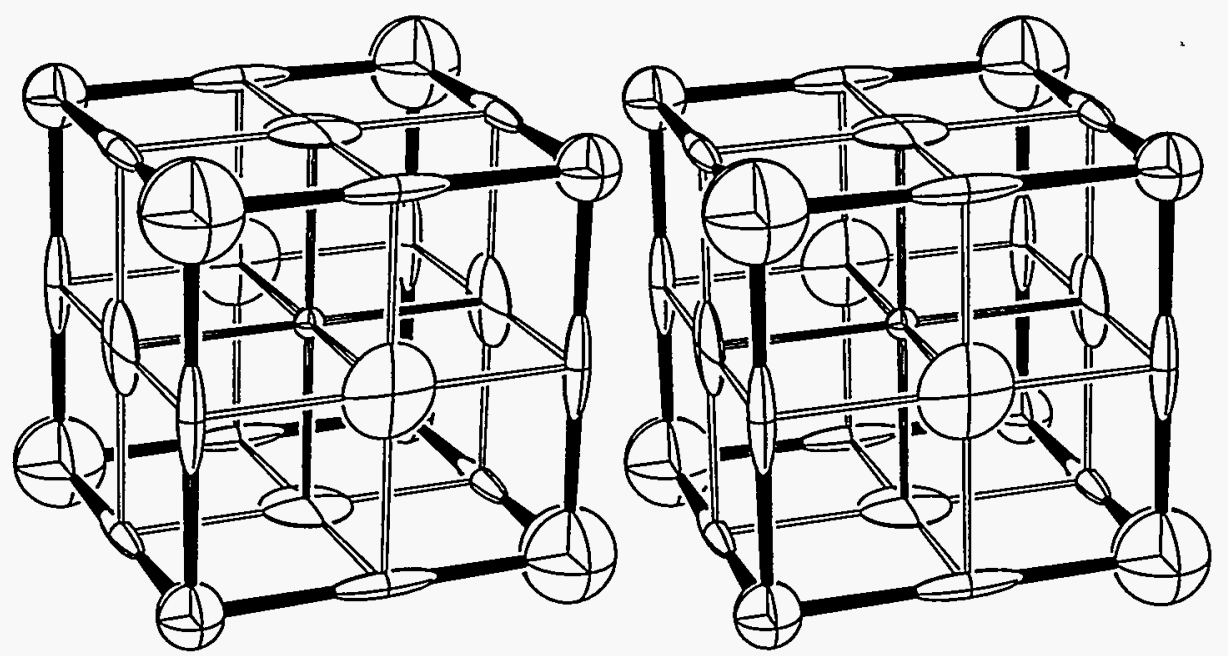

Fig. 7.9. Sodium chloride critical net.

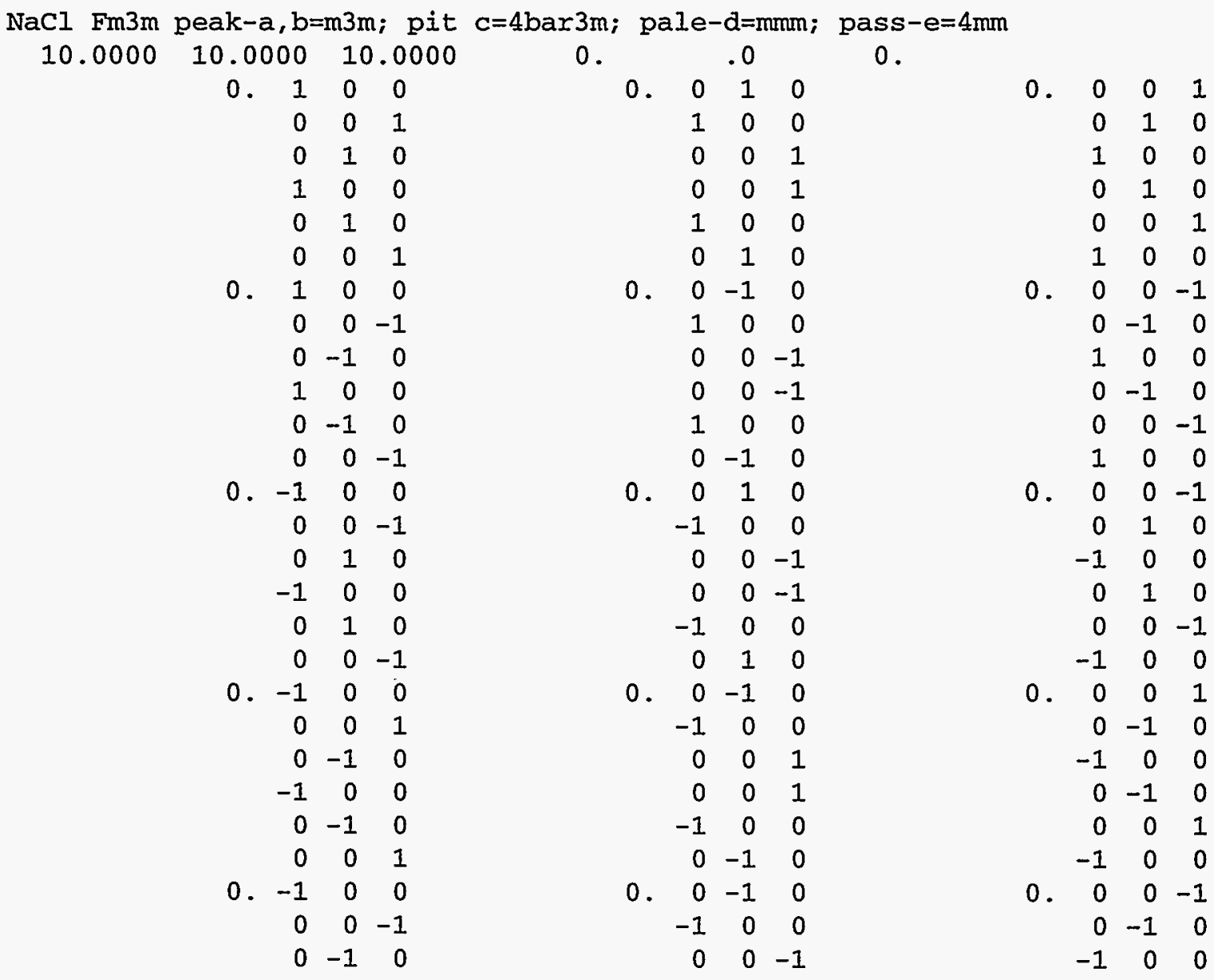


1

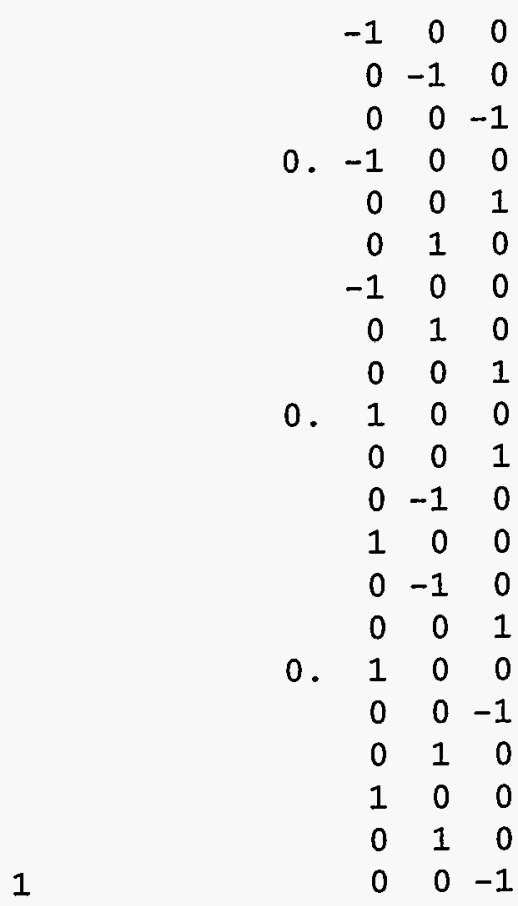

$\mathrm{Na}$

.10

$\mathrm{Na}$

.10

$\mathrm{Na}$

Na $\begin{array}{r}.10 \\ .10\end{array}$

b $\mathrm{Cl}$

b $\mathrm{Cl}$

b CI

b $\mathrm{Cl}$

.15

.05

$c$ Pit

.05

c Pit .05

c Pit

Pass

.18

Pass

Pass

.18

Pass $\begin{array}{lll}0 & 0 & -1\end{array}$

$\begin{array}{lll}-1 & 0 & 0\end{array}$

$0 \begin{array}{lll}0 & -1 & 0\end{array}$

0. $0 \begin{array}{lll}0 & 1 & 0\end{array}$

$\begin{array}{lll}-1 & 0 & 0\end{array}$

$0 \begin{array}{lll}0 & 0 & 1\end{array}$

$\begin{array}{lll}0 & 0 & 1\end{array}$

$\begin{array}{lll}-1 & 0 & 0\end{array}$

$0 \begin{array}{lll}0 & 1 & 0\end{array}$

0. $\quad 0 \begin{array}{rrr} & -1 & 0\end{array}$

$\begin{array}{lll}1 & 0 & 0\end{array}$

$\begin{array}{lll}0 & 0 & 1\end{array}$

$\begin{array}{lll}0 & 0 & 1\end{array}$

$\begin{array}{lll}1 & 0 & 0\end{array}$

$\begin{array}{lll}0 & -1 & 0\end{array}$

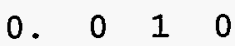

$\begin{array}{lll}1 & 0 & 0\end{array}$

$\begin{array}{lll}0 & 0 & -1\end{array}$

$\begin{array}{llll}0 & 0 & -1\end{array}$

100

010

.0

$\begin{array}{ll}.0 & .0\end{array}$

.0

.5

.0

.5

.5

.5

.5

0

.0

25

.25

.75

.75

.25

$.04 \quad 155501 \quad 555501$

75

$.04 \quad 155501 \quad 555501$

$04 \quad 155501 \quad 555501$

.5

.5

.0

$.0 \quad .0$

$.5 \quad .5$

$.5 \quad .0$

$.0 \quad .5$

$.25 \quad .25$

$.75 \quad .75$

$.25 \quad .75$

$\begin{array}{ll}.75 & .25\end{array}$

$.00 \quad .00$

$.50 \quad .00$

$.00 \quad .50$

.25

$\begin{array}{lll}0 & -1 & 0\end{array}$

$\begin{array}{lll}0 & 0 & -1\end{array}$

$\begin{array}{lll}-1 & 0 & 0\end{array}$

0. 0001

$\begin{array}{lll}0 & 1 & 0\end{array}$

$\begin{array}{lll}-1 & 0 & 0\end{array}$

$\begin{array}{lll}0 & 1 & 0\end{array}$

$\begin{array}{lll}0 & 0 & 1\end{array}$

$\begin{array}{lll}-1 & 0 & 0\end{array}$

0. $0 \begin{array}{lll} & 0 & 1\end{array}$

$\begin{array}{lll}0 & -1 & 0\end{array}$

100

$\begin{array}{lll}0 & -1 & 0\end{array}$

$\begin{array}{lll}0 & 0 & 1\end{array}$

$\begin{array}{lll}1 & 0 & 0\end{array}$

0. $000-1$

$\begin{array}{lll}0 & 1 & 0\end{array}$

$\begin{array}{lll}1 & 0 & 0\end{array}$

$\begin{array}{lll}0 & 1 & 0\end{array}$

$\begin{array}{lll}0 & 0 & -1\end{array}$

100

7

7

7

$\begin{array}{ll}75 & 7\end{array}$

$.50 \quad .50$

7

7

7

7

7

7

7

7

7 
104

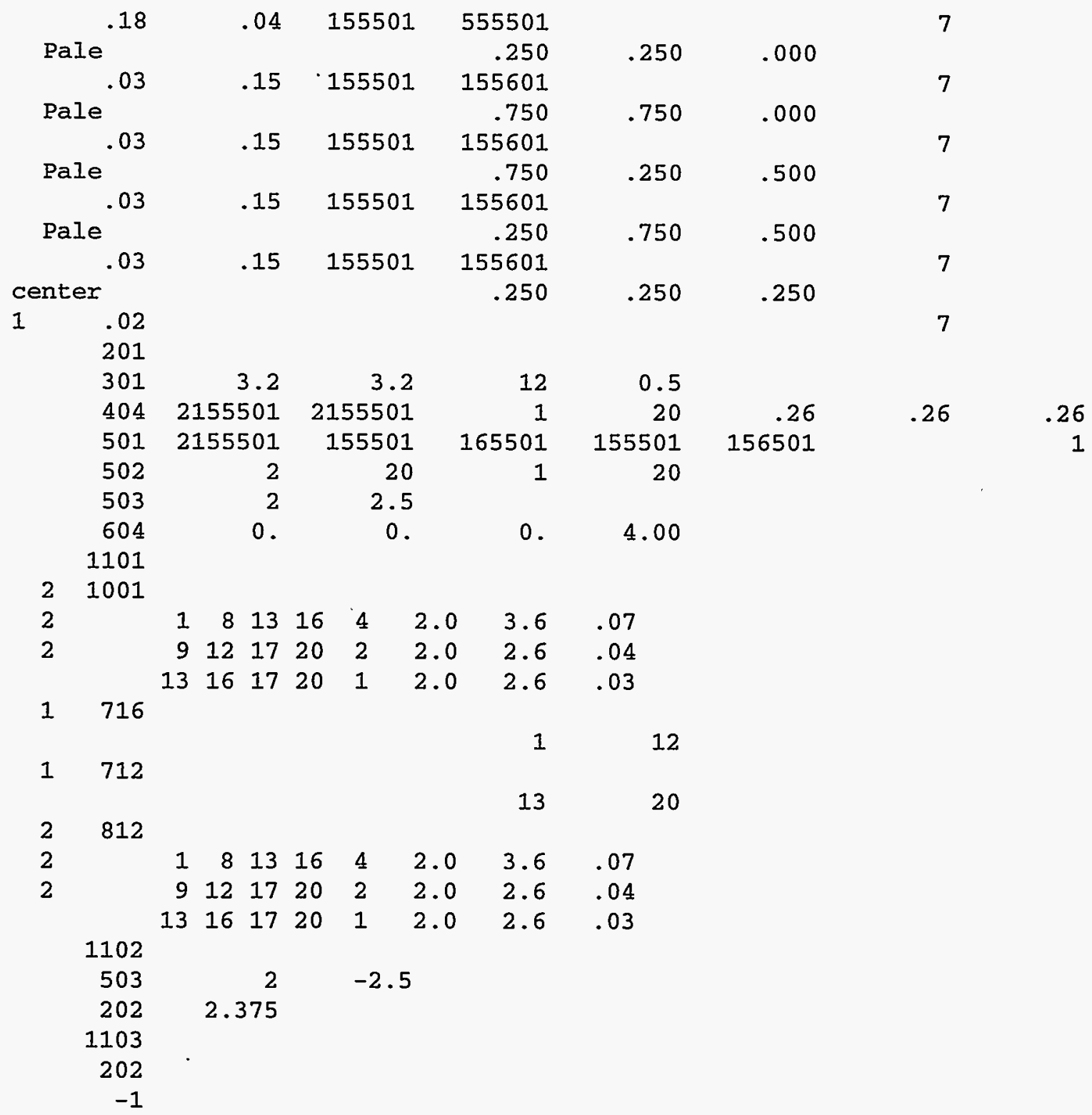




\section{REFERENCES}

1. C. K. Johnson, OR TEP: A FORTRAN Thermal-Ellipsoid Plot Program for Crystal Structure Illustrations, ORNL-3794 (Rev.), Union Carbide Corp., Oak Ridge Natl. Lab., June 1965.

2. C. K. Johnson, OR TEP-II: A FORTRAN Thermal-Ellipsoid Plot Program for Crystal Structure Illustrations, ORNL-5138, Union Carbide Corp., Oak Ridge Natl. Lab., March 1976.

3. Science Citation Index, Institute for Scientific Information, Inc., Philadelphia, 1995.

4. T. Hahn, ed., International Tables for Crystallography A, Kluwer Academic Publishers, Dordrecht, The Netherlands, 1995.

5. E. B. Fleischer, "X-Ray Structure Determination of Cubane," J. Am. Chem. Soc. 86, 38893890 (1964).

6. C. K. Johnson, "Generalized Treatments for Thermal Motion," pp. 132-160 in Thermal Neutron Diffraction, ed. B. T. M. Willis, Oxford University Press, London, 1970.

7. R. R. Holmes and R. M. Deiters, "Anisotropic Thermal Motion of Trigonal Bipyramidal Molecules from Spectroscopic Data. Pentacoordinated Molecules. XIII," J. Chem. Phys. 51, 4043-4054 (1969).

8. J. Heading, Matrix Theory for Physicists, Longmans, Green and Co., London, 81-106, 1958.

9. C. E. Springer, Geometry and Analysis of Projective Spaces, Freeman and Co., San Francisco, 1964.

10. J. A. Todd, Projective and Analytical Geometry, Pitman Pub. Corp., New York, 1946.

11. G. A. Korn and T. M. Korn,Mathematical Handbook for Scientists and Engineers, McGrawHill Book Company, New York, 1961.

12. J. T. Rule, "Stereoscopic Drawings," J. Opt. Soc. Am. 28, 313-322 (1938).

13. B. G. Saunders, "Stereoscopic Drawing by Computer-Is it Orthoscopic?," Appl. Opt. 7, 1499-1504 (1968).

14. S. S. Wilks, Mathematical Statistics, Wiley, New York, 1962.

15. H. Cramer, Random Variables and Probability Distributions, Cambridge University Press, London, 1962.

16. K. S. Miller, Multidimensional Gaussian Distributions, John Wiley \& Sons, New York, 1964.

17. W. C. Hamilton, Statistics in Physical Science, Ronald Press, New York, 1964.

18. E. Lukacs and R. G. Laha, Applications of Characteristic Functions, Hafner Publishing Co., New York, 1964. 
19. R. S. Burington and D. C. May, Handbook of Probability and Statistics with Tables, 2nd ed., McGraw-Hill, New York, 1970.

20. D. B. Owen, Handbook of Statistical Tables, Addison-Wesley, Reading, Mass., 1962.

21. J. Waser, "The Anisotropic Temperature Factor in Triclinic Coordinates," Acta Cryst. 8, 731 (1955).

22. W. R. Busing and H. A. Levy, "Determination of the Principal Axes of the Anisotropic Temperature Factor," Acta Cryst. 11, 450-451 (1958).

23. D. W. J. Cruickshank et al., "Crystallographic Calculations on the Ferranti Pegasus and Mark I Computers," pp. 32-78 in Computing Methods and the Phase Problem in X-Ray Crystal Analysis, ed. R. Pepinsky, J. M. Robertson, and J. C. Speakman, Pergamon Press, New York, 1961.

24. C. K. Johnson, unpublished data.

25. A. Elliott and B. R. Malcolm, "Chain Arrangement and Sense of the $\alpha$-Helix in Poly-LAlanine Fibres," Proc. Roy. Soc. London A249, 30-41 (1959).

26. A. Zalkin, J. D. Forrester, D. H. Templeton, S. M. Williamson, and C. W. Koch, "Potassium Perxenate Nonahydrate," J. Am. Chem. Soc. 86, 3569-3571 (1964). 


\section{APPENDIX A}

\section{ORTEP-III SUBPROGRAMS}

\begin{tabular}{|c|c|}
\hline FUNCTION ARCCOS(X) & $\begin{array}{l}\text { Computes } \theta \text {, the arc cosine of } X \text { in degrees; } 0 \leq \theta \leq \\
180^{\circ} \text {. }\end{array}$ \\
\hline $\operatorname{ATOM}(Q A, Z)$ & $\begin{array}{l}\text { Finds the triclinic coordinates } \mathbf{Z} \text { for the atom } \\
\text { described by the atom designator code QA. }\end{array}$ \\
\hline $\mathrm{AXEQB}(\mathrm{A} 1, \mathrm{X}, \mathrm{B} 1, \mathrm{JJJ})$ & $\begin{array}{l}\text { Solves the matrix equation } \mathbf{A 1} \mathbf{X}=\mathbf{B 1} \text { for } \mathbf{X} \text {. The } \\
\text { matrices } \mathbf{B 1} \text { and } \mathbf{X} \text { are }(3, \mathrm{JJJ}) \text { and } \mathbf{A 1} \text { is always } \\
(3,3) \text {. To invert } \mathbf{A 1} \text {, make } \mathbf{B 1} \text { an identity matrix. }\end{array}$ \\
\hline $\operatorname{AXES}(U, V, X, I T Y P E)$ & $\begin{array}{l}\text { Provides three orthogonal column vectors in } \mathbf{X}, \\
\text { each } 1 \AA \text { long, from the two vectors } \mathbf{U} \text { and } \mathbf{V} . \\
\text { ITYPE }>0: \text { Cartesian system } \\
\text { ITYPE <0: triclinic system } \\
\begin{aligned}|I T Y P E|=1: & \mathbf{X}_{1}=\mathbf{U} ; \mathbf{X}_{2}=(\mathbf{U} \times \mathbf{V}) \\
& \mathbf{X}_{3}=\mathbf{U} \times(\mathbf{U} \times \mathbf{V}) \\
|I T Y P E|=2: & \mathbf{X}_{1}=\mathbf{U} ; \mathbf{X}_{2}=(\mathbf{U} \times \mathbf{V}) \times \mathbf{U} ; \\
& \mathbf{X}_{3}=\mathbf{U} \times \mathbf{V} \\
\text { ITYPE }=0: & \text { same as type } 2 \text { except } \mathbf{U}=a \text { crystal } \\
& \text { axis, } \mathbf{V}=b \text { crystal axis. }\end{aligned}\end{array}$ \\
\hline $\mathrm{BOND}(\mathrm{Z1}, \mathrm{Z2}, \mathrm{NB}, \mathrm{NA} 1, \mathrm{NA} 2)$ & $\begin{array}{l}\text { Draws a bond, described by Format No. } 2 \text { trailer } \\
\text { card number NB, between two atoms. Zn is atom } \\
\text { designator code of atom } n \text {, and NA } n \text { is number of } \\
\text { atom } n \text { in ATOMS array. }\end{array}$ \\
\hline COLRXX (ICOLOR) & Sets plot color on "device" $x x$ to ICOLOR. \\
\hline CURSSC & Identifies atoms selected on screen display. \\
\hline DFLTS & Sets default values for items requested from user. \\
\hline $\operatorname{DIFV}(X, Y, Z)$ & $\begin{array}{l}\text { Performs the vector subtraction } \mathbf{X}-\mathbf{Y}=\mathbf{Z} . \mathbf{Z} \text { may } \\
\text { have the same location as } \mathbf{X} \text { or } \mathbf{Y} \text {. }\end{array}$ \\
\hline $\operatorname{DRAW}(W, \mathrm{DX}, \mathrm{DY}, \mathrm{NPEN})$ & $\begin{array}{l}\text { Interconnects ORTEP and the plot package. It also } \\
\text { prevents the pen from crossing the boundaries. If } \\
\text { the indicator ITLT in COMMON is zero, the array } \\
W \text { contains } x \text { and } y \text { in plotter coordinates. While } \\
\text { perspective lettering is being plotted, ITIIT } \neq 0 \text {; } \\
\text { and W contains } x, y, z \text { in Cartesian coordinates, } \\
\text { which will be rotated and projected by DRAW to } \\
\text { form plotter } x, y \text { coordinates. DX and DY are } \\
\text { added to the plotter } x \text { and } y \text {, respectively, before } \\
\text { the plot package is called. NPEN }=2 \text { for pen down } \\
\text { and } 3 \text { for pen up. }\end{array}$ \\
\hline
\end{tabular}




\begin{tabular}{|c|c|}
\hline EDITR & Controls ORTEP line editor. \\
\hline $\operatorname{EIGEN}(W, V A L U, V E C T)$ & $\begin{array}{l}\text { Determines the three eigenvalues VALU and the } \\
\text { three column eigenvectors VECT of the matrix W. } \\
\text { Indeterminate eigenvectors are replaced by zeros } \\
\text { and the fault indicator NG set to a negative value } \\
\text { (eigenvectors are assigned for the indeterminate } \\
\text { cases by PRELIM). }\end{array}$ \\
\hline END $X X$ & Terminates plotting on "device" $x x$. \\
\hline ERPNT $(T D, N)$ & $\begin{array}{l}\text { Prints error message when a fault is found. The } \\
\text { arguments identify the atom designator code TD } \\
\text { and the instruction } N \text { involved in the fault. The } \\
\text { fault indicator, NG, is in COMMON. }\end{array}$ \\
\hline EXITNG (ING) & $\begin{array}{l}\text { Prints fault indicator ING if abnormal termination } \\
\text { and stops program execution. }\end{array}$ \\
\hline F200 & Executes the 200 series instructions. \\
\hline$F 400$ & Executes the 400 series instructions. \\
\hline F500 & Executes all 500 series instructions. \\
\hline F600 & Executes all 600 series instructions. \\
\hline F700 & Executes all 700 series instructions. \\
\hline F800 & $\begin{array}{l}\text { Executes all } 800 \text { series instructions. Bonds to be } \\
\text { drawn are found by F } 800 \text {, then drawn by } \\
\text { subroutine BOND. }\end{array}$ \\
\hline F900 & Executes all 900 series instructions. \\
\hline F1000 & Executes the 1001 instruction. \\
\hline FUNCTION IEND (STRING) & $\begin{array}{l}\text { Returns the position of the last non-space character } \\
\text { in a character STRING. }\end{array}$ \\
\hline INITXX & Initializes plotting on "device" $x x$. \\
\hline LAP500 (NTYPE) & $\begin{array}{l}\text { Sorts the ATOMS array, then calculates the } \\
\text { projected outline ellipses for all atoms in the } \\
\text { ATOMS array. The ellipses are stored in the } \\
\text { CONIC array along with the minima and maxima } \\
\text { in } x \text { and } y \text { for a rectangle enclosing each ellipse. If } \\
\text { NTYPE < } 0 \text {, previous overlap information is } \\
\text { cleared. }\end{array}$ \\
\hline
\end{tabular}




\begin{tabular}{|c|c|}
\hline LAP700 (NA, ICQ) & $\begin{array}{l}\text { Finds the atoms that overlap a given atom to be } \\
\text { drawn. The routine first checks the bounding } \\
\text { rectangles for intersections, then forms the cubic } \\
\text { discriminant from the quadratic descriptions of the } \\
\text { two projection ellipses. The discriminant provides a } \\
\text { specification for complete overlap, partial overlap, } \\
\text { or no overlap. A list of up to } 20 \text { interfering ellipses } \\
\text { is compiled. NA is the atom to be drawn. ICQ is set } \\
>0 \text { if overlap exists and }=-1 \text { if not. }\end{array}$ \\
\hline LAP800 (NA1, NA2, ICQ) & $\begin{array}{l}\text { Used in the "Projected Outline Storage Step" to } \\
\text { store the projected quadrangles for the bonds } \\
\text { specified by the trailer cards of the 1001, } 821 \text {, and } \\
822 \text { instructions. The routine also is used in the } \\
\text { "Area-Overlap Search Step" to find the projected } \\
\text { bond quadrangles that overlap a given bond to be } \\
\text { drawn. A list containing up to } 30 \text { interfering } \\
\text { quadrangles is compiled. NA1 and NA2 are the } \\
\text { two atoms of the bond. ICQ is set }>0 \text { if overlap } \\
\text { exists and = } 1 \text { if not. }\end{array}$ \\
\hline LAPAB (IQ, IA, ICQ, ITY) & $\begin{array}{l}\text { Finds the bonds that overlap an atom to be drawn } \\
\text { and the atoms that overlap a bond to be drawn. It is } \\
\text { used in the "Area-Overlap Search Step." ITY }>0 \\
\text { checks for atom, IA, over bond, IQ, and ITY }<0 \\
\text { checks for bond over atom. ICQ is set }>0 \text { if } \\
\text { overlap exists, = } 0 \text { for no overlap, and }<0 \text { for } \\
\text { hidden atom or bond. }\end{array}$ \\
\hline LAPCON (CON1, CON , Y, OVMR) & $\begin{array}{l}\text { Transforms conic, CON1, to plotter homogeneous } \\
\text { coordinate system, CON, with center at Y. OVMR } \\
\text { denotes overlap margin. }\end{array}$ \\
\hline LAPDRW ( $Y$, NPEN , NCQ) & $\begin{array}{l}\text { Checks each line segment to be drawn for } \\
\text { intersection with the interfering ellipses and } \\
\text { quadrangles and compiles a list of intersections. } \\
\text { The intersection list is sorted according to distance } \\
\text { along the line segment, and the intersection pattern } \\
\text { is analyzed to determine which subsegments are } \\
\text { visible and which are hidden. The line subsegments } \\
\text { are passed to the SCRIBE routine. Y is pen } \\
\text { position, NPEN denotes if pen is up or down, and } \\
\text { NCQ is set to NCOVER+NQOVER. }\end{array}$ \\
\hline $\begin{array}{l}\text { CHARACTER* }(*) \text { FUNCTION } \\
\text { MAKSYM (GP) }\end{array}$ & $\begin{array}{l}\text { Returns a character string representation (xyz } \\
\text { notation) of a symmetry operator stored in } \\
\text { ORTEP's internal representation in array GP. }\end{array}$ \\
\hline $\operatorname{MM}(X, Y, Z)$ & $\begin{array}{l}\text { Performs the matrix multiplication } \mathbf{X Y}=\mathbf{Z} \text {. The } \\
\text { location of } \mathbf{Z} \text { must be different from } \mathbf{X} \text { and } \mathbf{Y} \text {. }\end{array}$ \\
\hline
\end{tabular}




\begin{tabular}{|c|c|}
\hline $\operatorname{MV}(X, Y, Z)$ & $\begin{array}{l}\text { Performs the matrix-vector multiplication } \mathbf{X Y}=\mathbf{Z} \text {. } \\
\text { The location of } \mathbf{Z} \text { must be different from } \mathbf{X} \text { and } \mathbf{Y} \text {. }\end{array}$ \\
\hline NORM $(\mathrm{X}, \mathrm{Y}, \mathrm{Z}$, ITYPE $)$ & $\begin{array}{l}\text { Stores at } \mathbf{Z} \text { a vector (not necessarily a unit vector) } \\
\text { perpendicular to both } \mathbf{X} \text { and } \mathbf{Y} \text {. The sense of } \mathbf{Z} \text { is } \\
\text { that of the vector product } \mathbf{X} \times \mathbf{Y} \text {. } \\
\text { ITYPE }>0 \text { : Cartesian system } \\
\text { ITYPE } \leq 0 \text { : triclinic system }\end{array}$ \\
\hline NUMBUR (W, W2 , HGT , DIST, THT , ND) & $\begin{array}{l}\text { Converts number to character string for placement } \\
\text { on the drawing. W contains coordinates of the } \\
\text { lower left edge of the first character, W } 2 \text { is unused, } \\
\text { HGT is the height of the characters, DIST is the } \\
\text { number to be drawn, THT is the angle by which } \\
\text { the base line of the characters is to be rotated } \\
\text { counterclockwise from the positive } x \text { axis, and ND } \\
\text { is the number of digits to the right of the decimal } \\
\text { point. }\end{array}$ \\
\hline ORTEP & $\begin{array}{l}\text { ORTEP is the MAIN program and controlling } \\
\text { routine that decodes the ORTEP instructions. It } \\
\text { either executes the command directly or calls the } \\
\text { appropriate subroutine to execute the instruction. }\end{array}$ \\
\hline PAXES (DCODE, ITYPE) & $\begin{array}{l}\text { Stores the covariance (dispersion) matrix for the } \\
\text { thermal ellipsoid or its inverse matrix, which is the } \\
\text { matrix of coefficients in the quadratic form } \\
\text { describing the ellipsoid, in COMMON at } Q \text { for the } \\
\text { atom with atom designator code DCODE. } \\
\text { ITYPE > } 0 \text { for covariance matrix } \\
\text { ITYPE }<0 \text { for ellipsoid quadratic form matrix } \\
\text { IITYPE| = } 1 \text { based on triclinic system } \\
\text { IITYPEI = } 2 \text { based on working Cartesian system } \\
\text { IITYPE| = } 3 \text { based on reference Cartesian system }\end{array}$ \\
\hline $\operatorname{PENXx}(X, Y, I P E N)$ & $\begin{array}{l}\text { Controls pen movement on "device" } x x . X \text { is the } \\
\text { abscissa and } Y \text { is the ordinate expressed in inches. } \\
\text { IPEN=2: pen draws line as it moves } \\
\text { IPEN=3: pen moves without drawing line }\end{array}$ \\
\hline PENWXXX ( PENW) & $\begin{array}{l}\text { Sets pen thickness on "device" } x x \text { to PENW. } \\
\text { PENW is provided in thousandths of an inch. The } \\
\text { default is } 5 .\end{array}$ \\
\hline $\operatorname{PLOT}(X, Y$, IPEN) & $\begin{array}{l}\text { Calls the appropriate PEN } x x \text { routine for drawing } \\
\text { lines on "device" } x x \text {. Parameters are sent to } \\
\text { PENxx. }\end{array}$ \\
\hline $\operatorname{PLTXY}(X, Y)$ & $\begin{array}{l}\text { Calculates the plotter coordinates } Y \text { from the } \\
\text { unscaled Cartesian coordinates X. The distance to } \\
\text { the closest boundary of the plot is stored in the } \\
\text { variable EDGE in COMMON. }\end{array}$ \\
\hline
\end{tabular}




\begin{tabular}{|c|c|}
\hline PRELIM & $\begin{array}{l}\text { Performs all calculations to process (e.g., principal } \\
\text { axis transformations) and store the input } \\
\text { crystallographic parameters. }\end{array}$ \\
\hline PRIME & $\begin{array}{l}\text { "Primes the program" by initializing all the } \\
\text { "primer parameters". }\end{array}$ \\
\hline PROJ (D, DP , X, XO, VIEW, I1 , I2 , I3) & $\begin{array}{l}\text { Used to obtain an array, DP, of plotter coordinates } \\
\text { from a scaled array, D, of points described in } \\
\text { Cartesian coordinates. X, XO, and VIEW are } \\
\text { parameters involved in the projection, and I1, I2, I3 } \\
\text { are DO loop parameters for indexing through the } \\
\text { array. }\end{array}$ \\
\hline RADIAL (ND) & $\begin{array}{l}\text { Generates a "radial" array ( } D \text { in COMMON) of } \\
\text { points lying on an ellipse, given two conjugate } \\
\text { radius vectors of the ellipse in the array DA in } \\
\text { COMMON. From } 8 \text { to } 128 \text { points are generated } \\
\text { depending on the value of ND }(1 \leq N D \leq 5) \text {. }\end{array}$ \\
\hline $\begin{array}{l}\text { READIN (IU, CHEM, ID1, ID2 , X1, } \\
\mathrm{X} 2, \mathrm{X} 3, I T, I S, B 1, \mathrm{~B} 2, \mathrm{~B} 3, \mathrm{~B} 4, \mathrm{~B} 5 \\
\mathrm{~B} 6, \mathrm{BTYPE})\end{array}$ & $\begin{array}{l}\text { Reads atom parameters in any format from a file. } \\
\text { This subroutine may be modified by the user. See } \\
\text { Section } 4.5 \text { for a description of the parameters. }\end{array}$ \\
\hline RECYCLE & $\begin{array}{l}\text { Returns instruction pointer to } 201 \text { instruction and } \\
\text { zeroes ATOMS array. }\end{array}$ \\
\hline $\operatorname{SCRIBE}(Y, N P E N)$ & $\begin{array}{l}\text { Filters out the hidden line segments and passes the } \\
\text { visible line segments to the DRAW routine. }\end{array}$ \\
\hline SEARC & $\begin{array}{l}\text { Conducts an exhaustive (but educated) search to } \\
\text { find all points within a sphere or rectangular box. } \\
\text { Interatomic distances and angles are also calculated } \\
\text { for the } 100 \text { series. }\end{array}$ \\
\hline SIMBOL (W, W2, HGT, ITXT, THT, N) & $\begin{array}{l}\text { Processes character strings for placement on the } \\
\text { drawing. W contains coordinates of the lower left } \\
\text { edge of the first character, W } 2 \text { is unused, HGT is } \\
\text { the height of the characters, ITXT is the string to } \\
\text { be drawn, THT is the angle by which the base line } \\
\text { of the characters is to be rotated counterclockwise } \\
\text { from the positive } x \text { axis, and N is the number of } \\
\text { characters to be drawn. }\end{array}$ \\
\hline SPARE (INST) & $\begin{array}{l}\text { Expands the user supplied instruction set by } \\
\text { responding to any INST } \geq 12 \text {. INST }=\text { instruction } \\
/ 100 \text {. }\end{array}$ \\
\hline STOR (TD1) & $\begin{array}{l}\text { Stores atom with atom designator code TD1 in (or } \\
\text { removes atom from) the ATOMS array. Coordi- } \\
\text { nates in whichever system is in use are communi- } \\
\text { cated to STOR via array V1 of COMMON. }\end{array}$ \\
\hline
\end{tabular}




\begin{tabular}{|c|c|}
\hline TEPSYM (TXT, NUM, KK) & $\begin{array}{l}\text { Parses symmetry operator in character string } \\
\text { representation (xyz notation), TXT, and stores the } \\
\text { information in ORTEP's internal representation. } \\
\text { NUM is symmetry operator number, and KK is the } \\
\text { component number. }\end{array}$ \\
\hline $\operatorname{TMM}(X, Y, Z)$ & $\begin{array}{l}\text { Performs the matrix multiplication }\left(\mathbf{X}^{\mathrm{T}} \mathbf{Y}\right)^{\mathrm{T}}=\mathbf{Z} \text {. } \\
\text { The location of } \mathbf{Z} \text { must be different from } \mathbf{X} \text { and } \mathbf{Y} \text {. }\end{array}$ \\
\hline UINPUT (IN, NOUT) & $\begin{array}{l}\text { Controls user input. IN is input file device number, } \\
\text { and NOUT is output file device number. }\end{array}$ \\
\hline $\operatorname{UNITY}(\mathrm{X}, \mathrm{Z}, \mathrm{ITYPE})$ & $\begin{array}{l}\text { Makes the vector } \mathbf{Z} 1 \AA \text { long and parallel to } \mathbf{X} \text {. } \\
\text { The vectors } \mathbf{X} \text { and } \mathbf{Z} \text { may have the same location. } \\
\text { ITYPE }>0 \text { : Cartesian system } \\
\text { ITYPE < 0: triclinic system }\end{array}$ \\
\hline $\operatorname{VM}(Y, X, Z)$ & $\begin{array}{l}\text { Performs the vector-matrix multiplication } \mathbf{Y}^{\mathrm{T}} \mathbf{X}= \\
\mathbf{Z}^{\mathrm{T}} \text {. The location of } \mathbf{Z} \text { must be different from } \mathbf{Y} \\
\text { and } \mathbf{X} \text {. }\end{array}$ \\
\hline FUNCTION $\operatorname{VMV}(X 1, Q, X 2)$ & $\begin{array}{l}\text { Performs the vector-matrix-vector multiplication } \\
\mathbf{X} 1^{\mathrm{T}} \mathbf{Q} \mathbf{X} \mathbf{2}=\text { scalar. }\end{array}$ \\
\hline FUNCTION VV $(\mathrm{X}, \mathrm{Y})$ & $\begin{array}{l}\text { Performs the vector-vector multiplication } \mathbf{X}^{\mathrm{T}} \mathbf{Y}= \\
\text { scalar. }\end{array}$ \\
\hline$X Y Z$ (DQA , X, ITYPE) & $\begin{array}{l}\text { Returns in } X \text { coordinates for atom with atom } \\
\text { designator code DQA. } \\
\text { ITYPE }=0: \text { triclinic coordinates } \\
\text { ITYPE }=1 \text { or } 2: \text { working Cartesian system } \\
\text { coordinates } \\
\text { ITYPE }=3: \text { reference Cartesian system coordinates }\end{array}$ \\
\hline
\end{tabular}




\section{APPENDIX B}

\section{GLOSSARY OF VARIABLES IN ORTEP-II COMMONS}

\begin{tabular}{|c|c|c|}
\hline$*$ & UNNAMED & Main Common Block \\
\hline & $A(9)$ & $\begin{array}{l}\text { Direct crystal cell parameters, a, b, c, } \cos \alpha, \cos \beta, \cos \gamma \\
\alpha, \beta, \gamma \text {. }\end{array}$ \\
\hline & $A A(3,3)$ & Metric tensor $\mathbf{g}$ where $\mathrm{g}_{i j}=\mathrm{a}_{i} \cdot \mathrm{a}_{\mathrm{j}}$. \\
\hline & $\operatorname{AAREV}(3,3)$ & $\begin{array}{l}\text { Postfactor transformation matrix to convert coordinates } \\
\text { from triclinic to the reference Cartesian system. } \\
\text { AAREV = AA REFV. }\end{array}$ \\
\hline & $\operatorname{AAWRK}(3,3)$ & $\begin{array}{l}\text { Postfactor transformation matrix to convert coordinates } \\
\text { from triclinic to the working Cartesian system. } \\
\text { AAWRK = AA WRKV. }\end{array}$ \\
\hline & $\operatorname{AID}(3,3)$ & Identity matrix. \\
\hline & $R E A L \star 8$ AIN $(140)$ & $\begin{array}{l}\text { Array containing the input parameters of the current } \\
\text { ORTEP instruction. }\end{array}$ \\
\hline & REAL *8 ATOMID $(500)$ & Atom designator codes of atoms in ATOMS array. \\
\hline & ATOMS $(3,500)$ & $\begin{array}{l}\text { Temporary storage of atom coordinates in any of } \\
\text { several coordinate systems. }\end{array}$ \\
\hline & $\mathrm{BB}(3,3)$ & Reciprocal metric tensor. $\mathbf{B B}=\mathbf{A A}^{-1}$. \\
\hline $\mathbf{P}$ & BRDR & $\begin{array}{l}\text { Border (margin) width in inches extending inward } \\
\text { from plot boundary. }\end{array}$ \\
\hline & $C D(8,20)$ & $\begin{array}{l}\text { Holds the real values entered on a Format No. } 2 \text { trailer } \\
\text { card. Used in conjunction with } \mathrm{KD} \text { array. }\end{array}$ \\
\hline $\mathrm{P}$ & $\operatorname{CONT}(5)$ & Constants used in subroutine RADIAL. \\
\hline & $D(3,130)$ & $\begin{array}{l}\text { Array in which three-dimensional points on an ellipse } \\
\text { are stored by RADIAL. }\end{array}$ \\
\hline & $\mathrm{DA}(3,3)$ & $\begin{array}{l}\text { Transmits conjugate vectors to RADIAL. Also used for } \\
\text { temporary storage. }\end{array}$ \\
\hline $\mathbf{P}$ & DISP & Displacement parameter for retracing. \\
\hline & $\mathrm{DP}(2,130)$ & $\begin{array}{l}\text { Array in which two-dimensional points for ellipse are } \\
\text { stored after projection. }\end{array}$ \\
\hline & EDGE & $\begin{array}{l}\text { Distance in inches from a projected point to the closest } \\
\text { boundary. Set in PLTXY. }\end{array}$ \\
\hline
\end{tabular}




\begin{tabular}{|c|c|c|}
\hline $\mathbf{P}$ & FORE & $\begin{array}{l}\text { Cosine of critical angle between bond and Cartesian } z \\
\text { axis vectors for perspective bond distance labels. At } \\
\text { smaller angles, the labels, produced from subroutine } \\
\text { BOND, are drawn without perspective to prevent } \\
\text { excessive foreshortening. }\end{array}$ \\
\hline & $\operatorname{FS}(3,3,96)$ & $\begin{array}{l}\text { Rotation matrices for input symmetry operators based } \\
\text { on triclinic system. Used with TS array. }\end{array}$ \\
\hline & IN & Logical unit number of input file. \\
\hline $\mathrm{P}$ & ITILT & $\begin{array}{l}\text { Indicator used to signal subroutine DRAW, whether or } \\
\text { not to do perspective labeling. }\end{array}$ \\
\hline & $\mathrm{KD}(5,20)$ & $\begin{array}{l}\text { Holds the integer values entered on a Format No. } 2 \\
\text { trailer card. Used in conjunction with CD. }\end{array}$ \\
\hline $\mathbf{P}$ & LATM & Number of entries in ATOMS array. \\
\hline & NATOM & Number of input atoms. \\
\hline $\mathbf{P}$ & NCD & $\begin{array}{l}\text { Number of Format No. } 2 \text { trailer cards for an instruc- } \\
\text { tion. }\end{array}$ \\
\hline $\mathbf{P}$ & NG & Fault Indicator value. \\
\hline & NJ & Instruction number/100. \\
\hline & NJ2 & $\begin{array}{l}\text { Last two decimal digits of the instruction number } \\
\text { (instruction }=\mathrm{NJ} \times 100+\mathrm{NJ} 2 \text { ). }\end{array}$ \\
\hline & NOUT & Logical unit number of text output file. \\
\hline & NSR & Logical unit number of scratch file. \\
\hline & NSYM & Number of input symmetry operators. \\
\hline $\mathrm{P}$ & ORGN (3) & $\begin{array}{l}\text { Triclinic coordinates for the atom that is the origin of } \\
\text { the drawing (i.e., on the optic axis for the projection). }\end{array}$ \\
\hline & $\operatorname{PAC}(3,5)$ & $\begin{array}{l}\text { A } 3 \times 3 \text { matrix produced by subroutine PAXES and } \\
\text { made up of three orthonormal principal axis column } \\
\text { vectors, based on either the working or reference } \\
\text { Cartesian system. Columns } 4 \text { and } 5 \text { are used in } \\
\text { subroutine F700 to duplicate columns } 1 \text { and } 2 \text { for ease } \\
\text { in indexing. }\end{array}$ \\
\hline & $\operatorname{PAT}(3,3)$ & $\begin{array}{l}\text { A matrix produced by subroutine PAXES and } \\
\text { composed of three principal axis column vectors each } \\
1 \AA \text { long, based on the triclinic system. }\end{array}$ \\
\hline & $Q(3,3)$ & $\begin{array}{l}\text { A matrix produced by subroutine PAXES. Contains } \\
\text { either the dispersion matrix or its inverse, based on } \\
\text { either the working or reference Cartesian systems. }\end{array}$ \\
\hline
\end{tabular}




\begin{tabular}{|c|c|c|}
\hline & $\operatorname{REFV}(3,3)$ & $\begin{array}{l}\text { A matrix made up of three orthogonal column vectors, } \\
\text { each } 1 \AA \text { long, based on the triclinic system. This is the } \\
\text { base vector triplet for the reference Cartesian } \\
\text { coordinate system. The transpose is the postfactor } \\
\text { transformation matrix for converting coordinates from } \\
\text { the reference orthogonal system to the triclinic system. } \\
\mathbf{R E F V}^{\mathrm{T}}=\mathbf{A A R E V}{ }^{-1} \text {. }\end{array}$ \\
\hline \multirow[t]{2}{*}{$\mathbf{P}$} & RES (4) & $\begin{array}{l}\text { Regulates the resolution of the plotting of a given } \\
\text { ellipse as a function of the longest principal axis } x \text { in } \\
\text { the given ellipsoid of the scaled model. } \\
\qquad \begin{array}{ll}x \geq \operatorname{RES}(1) & \text { 128-point ellipse } \\
\operatorname{RES}(1)>x \geq \operatorname{RES}(2) & \text { 64-point ellipse } \\
\operatorname{RES}(2)>x \geq \operatorname{RES}(3) & \text { 32-point ellipse } \\
\operatorname{RES}(3)>x & \text { 16-point ellipse } \\
\operatorname{RES}(4) & \text { not used }\end{array}\end{array}$ \\
\hline & RMS (5) & $\begin{array}{l}\text { The rms displacements along the principal axes in } \\
\text { arrays PAC and PAT. }\end{array}$ \\
\hline $\mathrm{P}$ & SCAL1 & $\begin{array}{l}\text { The scale of the model in inches per Angstrom before } \\
\text { projection. }\end{array}$ \\
\hline$P$ & SCAL2 & $\begin{array}{l}\text { The scale factor ratio that sets the ellipsoid scale relative } \\
\text { to SCAL1. }\end{array}$ \\
\hline $\mathrm{P}$ & SCL & SCL $=$ SCAL1 1 SCAL2 \\
\hline $\mathrm{P}$ & $\operatorname{SYMB}(3,3)$ & $\begin{array}{l}\text { A rotation matrix based on the angle THETA, which } \\
\text { is set by instruction } 302 \text {. }\end{array}$ \\
\hline $\mathbf{P}$ & TAPER & $\begin{array}{l}\text { The exaggerated bond taper parameter. The top and } \\
\text { bottom ends of a bond have radii: RADIUS }=1 . \pm \\
\text { TAPER } \times \text { T } 6 \text { where T } 6=\text { lcosine of angle between } \\
\text { bond and } z \text { axis of Cartesian systeml. }\end{array}$ \\
\hline \multirow[t]{4}{*}{$\mathrm{P}$} & THETA & $\begin{array}{l}\text { Angle in degrees between plot } x \text { axis and lettering base- } \\
\text { line vector. }\end{array}$ \\
\hline & CHARACTER*4 TITLE (18) & Alphanumeric job title storage. \\
\hline & CHARACTER*4 TITLE2 (18) & $\begin{array}{l}\text { Alphanumeric information storage for Format No. } 3 \\
\text { trailer card. }\end{array}$ \\
\hline & $\operatorname{TS}(3,96)$ & $\begin{array}{l}\text { Translation vector for each input symmetry operator. } \\
\text { Used with FS array. }\end{array}$ \\
\hline \multirow[t]{3}{*}{$P$} & VIEW & Viewing distance in inches. \\
\hline & $\operatorname{VT}(3,4)$ & $\begin{array}{l}\text { Perspective title rotation matrix and translation vector. } \\
\text { Also used for temporary storage. }\end{array}$ \\
\hline & $\mathrm{V} 1(4)$ & $\begin{array}{l}\text { Array to transfer data to subroutine STORE. Also used } \\
\text { for temporary storage. }\end{array}$ \\
\hline
\end{tabular}




\begin{tabular}{|c|c|c|}
\hline & $\begin{array}{l}\mathrm{V} 2(3), \mathrm{V} 3(3), \mathrm{V} 4(3) \\
\mathrm{V} 5(3), \mathrm{V} 6(3)\end{array}$ & Temporary storage. \\
\hline & $\operatorname{WRKV}(3,3)$ & $\begin{array}{l}\text { Same definition as for REFV except that this one is for } \\
\text { working Cartesian system. } \text { WRKV }^{\mathrm{T}}=\mathbf{A A W K}^{-1} \text {. }\end{array}$ \\
\hline $\mathbf{P}$ & XLNG (3) & $\begin{array}{l}\text { Elements } 1 \text { and } 2 \text { are } x \text { and } y \text { plot dimensions. Element } \\
3 \text { is not used. }\end{array}$ \\
\hline $\mathbf{P}$ & xo(3) & $\begin{array}{l}\text { Elements } 1 \text { and } 2 \text { denote the position in plotter } \\
\text { coordinates (in inches) where ORGN is placed. Element } \\
3 \text { is used to transfer } z \text { coordinates to subroutine DRAW } \\
\text { when perspective lettering is used. }\end{array}$ \\
\hline & $\mathrm{XT}(3)$ & $\begin{array}{l}\text { Triclinic coordinates for an atom position are placed } \\
\text { here by subroutine XYZ. }\end{array}$ \\
\hline & DFL & Default Values for User Input \\
\hline & CHARACTER *60 ATOMFI & $\begin{array}{l}\text { Default name of file containing atom parameters used. } \\
\text { by subroutine READIN. }\end{array}$ \\
\hline & CHARACTER $* 4$ EXT & Default filename extension for ORTEP output. \\
\hline & FPAPLEN & Default page length for drawing \\
\hline & IDRAW & Default drawing destination indicator. \\
\hline & CHARACTER *60 INFILE & Default input file name. \\
\hline & IORIENT & Default orientation of drawing \\
\hline & IOUT & Default ORTEP text output logical unit number. \\
\hline & NS & Output Drawing Parameters \\
\hline & NDRAW & $\begin{array}{l}\text { ORTEP drawing destination indicator. } \\
\text { NDRAW=0: none } \\
\text { NDRAW=1: screen } \\
\text { NDRAW=2: Postscript file } \\
\text { NDRAW=3: HPGL file } \\
\text { NDRAW=9: Reserved for future use }\end{array}$ \\
\hline & NORIENT & Orientation of drawing. \\
\hline & NPF & Logical unit number of drawing output file. \\
\hline & NVAR & Temporary storage. \\
\hline & OLAP & Overlap Correction Variables \\
\hline & $\operatorname{CONIC}(7,500)$ & $\begin{array}{l}\text { Overlap correction ellipses describing intersection of } \\
\text { enveloping cones with drawing plane. }\end{array}$ \\
\hline
\end{tabular}




\begin{tabular}{|c|c|c|}
\hline & $\operatorname{COVER}(6,20)$ & $\begin{array}{l}\text { Stores up to } 20 \text { overlapping ellipses for an atom or } \\
\text { bond being drawn. }\end{array}$ \\
\hline & $\mathrm{KC}(20)$ & Which ellipses overlap the atom or bond being drawn. \\
\hline & $\mathrm{KQ}(30)$ & $\begin{array}{l}\text { Which quadrangles overlap the atom or bond being } \\
\text { drawn. }\end{array}$ \\
\hline & NCONIC & $\begin{array}{l}\text { Total number of projected ellipses stored for overlap } \\
\text { calculations. }\end{array}$ \\
\hline & NCOVER & $\begin{array}{l}\text { Number of projected ellipses over an atom or bond to } \\
\text { be drawn. }\end{array}$ \\
\hline & NQOVER & $\begin{array}{l}\text { Number of quadrangles over an atom or bond to be } \\
\text { drawn. }\end{array}$ \\
\hline & NQUAD & $\begin{array}{l}\text { Total number or projected bond quadrangles for } \\
\text { overlap calculations. }\end{array}$ \\
\hline & OVMRGN & Overlapping element margin. \\
\hline & $\operatorname{QOVER}(3,4,30)$ & $\begin{array}{l}\text { Stores up to } 30 \text { overlapping bond quadrangles for } \\
\text { atom or bond being drawn. }\end{array}$ \\
\hline & $\operatorname{QUAD}(9,600)$ & $\begin{array}{l}\text { Overlap correction bond quadrangles projected onto } \\
\text { drawing plane. }\end{array}$ \\
\hline & $\operatorname{SEGM}(50,2)$ & $\begin{array}{l}\text { Visible segments of an ellipsoid or bond element to be } \\
\text { drawn. }\end{array}$ \\
\hline & PARMS & Input Atom Parameters \\
\hline & CHARACTER *8 CHEM (505) & Names for input atoms. \\
\hline & $\mathrm{EV}(3,505)$ & $\begin{array}{l}\text { Root-mean-square displacements for each principal } \\
\text { axis of each input atom. }\end{array}$ \\
\hline & INTEGER*2 IDENT $(2,505)$ & Two feature identifiers for each input atom. \\
\hline $\mathrm{P}$ & MAXATM & Array size for input atoms. (Currently 505.) \\
\hline & $P(3,505)$ & Triclinic positional coordinates for the input atoms. \\
\hline & $\mathrm{PA}(3,3,505)$ & $\begin{array}{l}\text { Matrices for each input atom made up of three } \\
\text { orthogonal column eigenvectors each } 1 \AA \text { long, based } \\
\text { on the triclinic system (principal axis vectors). }\end{array}$ \\
\hline & PS & Encapsulated Postscript Output Parameters \\
\hline & IXMIN & Minimum $x$ value of illustration. \\
\hline & IXMAX & Maximum $x$ value of illustration. \\
\hline
\end{tabular}




\begin{tabular}{|l|l|l|}
\hline IYMIN & Minimum $y$ value of illustration. \\
\hline IYMAX & Maximum $y$ value of illustration. \\
\hline IXT & Page translation along $x$. \\
\hline QUEUE & Page translation along $y$. \\
\hline CHARACTER 73 HQUE (96) & Original instruction set as read from input file. \\
\hline CHARACTER*73 INQ & Next instruction held in memory to be processed. \\
\hline NBACK & $\begin{array}{l}\text { Number of lines in original instruction set as read from } \\
\text { input file. }\end{array}$ \\
\hline & NED & Logical unit number of temporary file used by editor. \\
\hline NEXT & $\begin{array}{l}\text { Line number of next instruction held in memory to be } \\
\text { processed. }\end{array}$ \\
\hline & NQUE & Current number of instruction lines held in memory. \\
\hline CHARACTER 73 QUE $(96)$ & Instruction lines held in memory. \\
\hline TRFAC & Plot Translation Factors \\
\hline XTRANS & Shift of plot origin along $x$-axis. \\
\hline YTRANS & Shift of plot origin along $y$-axis. \\
\hline
\end{tabular}

*Letter "P" indicates Prime Parameter (i.e., initialized in subroutine PRIME). 
APPENDIX C

ORTEP-III FORTRAN SOURCE CODE LISTING 


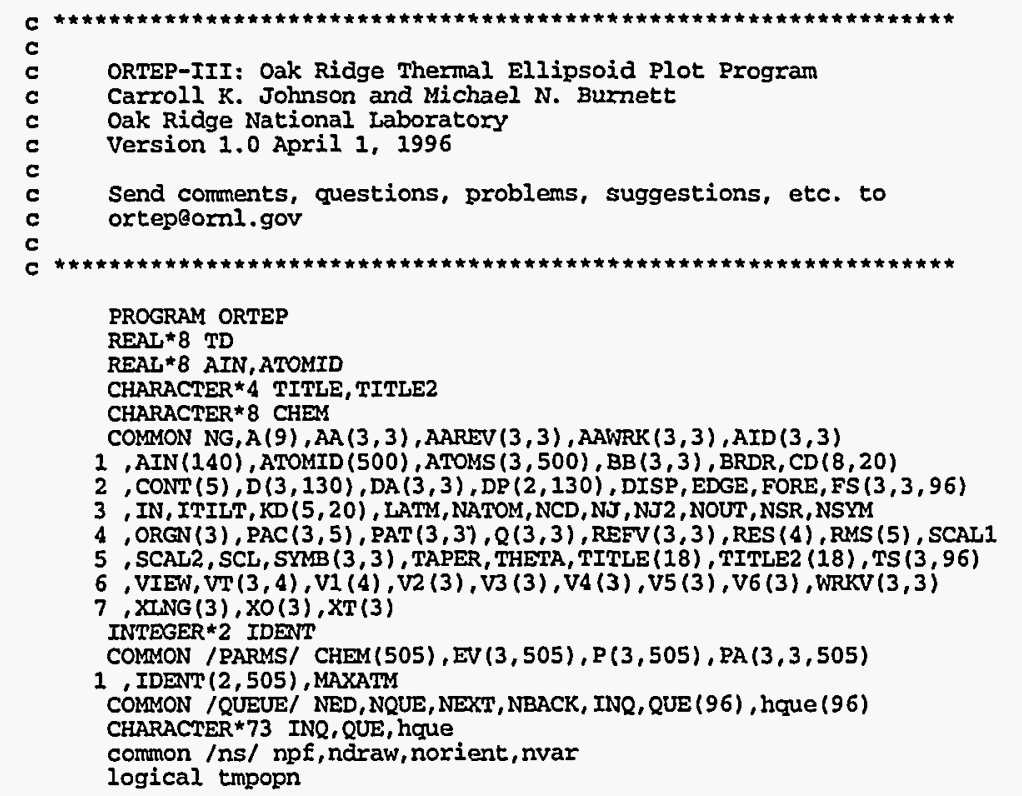

c $* \star \star$ Drawing Output Options

c $* * *$ ndraw $=0$ : no drawing output

c $* * *$ ndraw $=1$ : Screen output

$c * * *$ ndraw $=2$ : Postscript file
$c^{* * *}$ ndraw 3 : HPGL file output

$c * * \star$ Logical Unit Numbers $* * \star$

$c^{* * *} 15,16$ are used in subroutine EDITR

in) is used in subroutine PRELIM

*** NouT is set in subrouthe UTNPT

IN $=3$
NED $=7$
NSR $=8$
NPE $=10$

call uinput(in, nout)

2 CALL PRIME

$c$ *k* open ORTEP scratch file $* * *$

if already open, close it first $\star \star \star$

inquire (NSR, open

if (tmpopn) close(NSR)

open (NSR, status=' scratch' , form= ' unformatted' )

c *** open a temporary file - needed by the editor *** inquire (NED, opened=tmpopn) if (tmpopn) close(NED)

open (NED, status=' scratch')

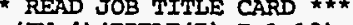

FORMAT (18A4)

IF (NOUT.GE. O)

$\operatorname{TITLE}(I), I=1,18$

(NOUT, 6 ) (TITLE(I) , I=1, 18)

FORMAT (1HO, 10X,18A4)

CALI PRELIM

IF (NOUT.GE.0)

\&WRITE (NOUT, 6) (TITLE(I), I=1, 18)

READ (IN, 2012, END=2015, ERR=3000) QUE (NOUE)

if (que (nque) $(1: 1)$. eq. '\#') go to 2010

hque (nque) =que (nque)

if (que (nque) $(4: 9)$. eq.

2012 FORMAT (A72)

IF (NQUE. LT . 96) GO TO 2005

2015 NQUE $=$ NQUE-

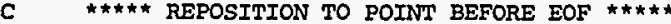
BACKSPACE IN

2020 NBACK=NQUE

NEXT $=1$

GO TO 507

ISAVE $=0$

8 DO $10 \mathrm{~J}=1,140$

11 FIN(J) $=0$.

FORMAT (13, 15,7515

4 FORMAT (1H 9X,7D15.7)

C $* \star \star \star \star *$ READ NEW INSTRUCTION CARD $* * \star \star * *$ $\mathrm{NCD}=0$

$16 \begin{aligned} & \mathrm{N} 1=-6 \\ & \mathrm{~N} 1=\mathrm{N} 1+7\end{aligned}$

$16 \mathrm{~N} 1=\mathrm{N} 1+7$

IF (ISAVE) $22,18,18$

18 INQ $=$ QUE (NEXT)

NEXT $=$ NEXT +1 READ (INQ, 12) IIC, NF, (AIN (I), I=N1,N2)

20 WFISE (NSR) IIC, NF, (AIN (I) , I=N1, N2)

2 READ (NSR) IIC, NF, ( $(A I N(I), I=N 2, N 2)$

IF (IIC) $7,24,24$

26 IF (NOUT.GE.0)

SWWRITE (NOUT, 11) NE

NE1 $=$ NF

c***

** Iun editor?

if (next. It.nque) go to 8 
$\operatorname{IF}(\mathrm{NF} 1+2) 2,2,3000$

CCC IF (NOUT.GE.0)

CCC EWRITE (NOUT, 14) (AIN (I), I=N1, N2)

32 IIC $=$ IIC+1

Go To $(90,16,38,50)$, IIC

33 FORMAT (I3, 6X,5I3,8

34 FORMAT $(613,8 \mathrm{E} 12.5)$ (2)

c $* \pi \star \star \star$ READ FORMAT 2 TRAILER CARDS $* \star \star \star * *$

$38 \mathrm{NCD}=\mathrm{NCD}+1$

IF (ISAVE) $44,40,40$

40 INQ $=$ QUE (NEXT)

NEXT $=$ NEXT+1

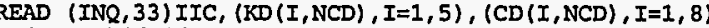

IF (ISAVE) $46,46,42$

KD (I,NCD) , I=1,5) , (CD (I,NCD) , I=1, 8$)$

44 READ (NSR) IIC, (KD (I, NCD) , $I=1,5),(C D(I, N C D), I=1,8$ )

IF (NOUT.GE.0)

(NOUT, 35) $(K D(I, N C D), I=1,5),(C D(I, N C D), I=1,8)$

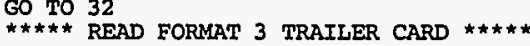

50 IF (ISAVE) $52,54,54$

LE2 (I), I=1, 18

GO TO 55

NEXT $=$ NEXT +1

READ (INQ, 4) (TITLE2 (I), I=1, 18)

55 IF (NOUT. GE. O)

SWRITE (NOUT, 5 ) (MITLE2 (I), I=1, 18

6 WRITE (NSR) (TTTLE2 (I

C $* \star \star \star \star *$ EXECUTE INSTRUCT) $1=1,18$ )

$90 \mathrm{NJ}=\mathrm{N} F 1 / 100$

$N J 2=N F 1-N N^{\star} 100$

NJ3 $=$ MOD $(N J 2,10)$

92 IF (NJ-12) $98,92,92$

CALI SPARE (NE

4 CALL ERPNT $(0 . D 0, N F 1)$

Go To 8

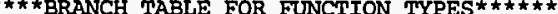

$98 \mathrm{GO} T \mathrm{TO}(100,200,300,400,500,600,700,800,900,1000,1100)$, NJ

C

100 GO TO $(101,101,104,104,101,101,94)$, NJ2

$$
\text { GO TO } 8
$$

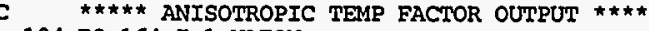

104 DO 164 I=1, NATOM

1F (MOD $(x, 14)-1) 134,114,134$

14 IF (NOUT.GE. 0 )

WRITE (NOUT, 6) (TITLE (J), J=1, 18)

IF (NOUT.GE.0)

129 FORMAT (1H010X, 4HATOM3X, 16HRMS DISPLACEMENT3X, 31HROW VECTORS, BASED 1 ON REFERENCE17X, 29HPROBABILITY COVARIANCE MATRIX)

$134 \mathrm{TD}=55501 .+\mathrm{FLOAT}(\mathrm{I}) \star 100000$

$$
\text { CALL PAXES (TD, - } 3 \text { ) }
$$

144 CALL ERPNT (TD, 104
149 FORMAT (1HO, 10X, A6, F10.6, 6X, 3F12.7, 10X, 3F12.7)

IF (NOUT.GE. G)

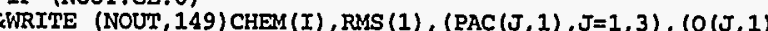

$1, J=1,3$

QWRITE (NOUT, 159) (RMS (K), (PAC $(J, K), J=1,3),(Q(J, K), J=1,3)$ $1, K=2,3$ )

59 FORMAT (1H , 16X, F10.6, 6X, 3F12.7, 10X, 3F12.7)

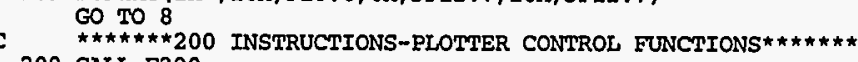
CALL F200

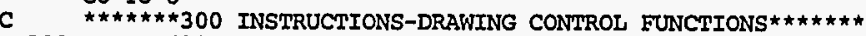

300 GO TO $(301,302,303,304,94)$, NJ2

301 IF (ATN (1) 321 DIMENSION

301 IF (AIN (1)) 321,

$321 \operatorname{IF}(A \operatorname{IN}(2)) 341,341,331$

331 XI (AIN) (2) 341,3

341 IF (AIN (3) ) 361,351,351

351 VIEW=AIN(3)

361 IF (AIN (4)) 381, 381, 371

371 BRDR=AIN $(4)$

81 IF (NOUT.GE.0)

1) XING (2) BRDR

作 $112 \mathrm{H}$ IN. MARGIN)

IF (NOUT'.GE.0)

99 FORMAT (1H , 10X, 13HVIEW DISTANCE, F7,3,7H INCHES)

T1 $=$ THETA ${ }^{\star}$. 01745329252

$\operatorname{SINTH}=\operatorname{SIN}(\mathrm{TI})$

DO $312 \quad J=1,9$

$312 \operatorname{SYMB}(J, 1)=0$.

STMB $(1,1)=$ COSTH

$\operatorname{STMB}(3,3)=1$.

$\operatorname{SYMB}(2,1)=\operatorname{STNTH}$

$\operatorname{SYMB}(1,2)=-S I N T H$

IF (NOUT.GE.0)

319 FORMAT (1HO10X, 44HREGULAR TITLE AND SYMBOL ROTATION IN DEGREESF8.2) GO TO 8 .

303 DISP=AIN (1)
IF (NOUT.GE.

\&WRITE (NOUT, 313) DISP

313 FORMAT (1H0, 10X, 22HRETRACE DISPLACEMENT $=$, F7 .4, 5H INCH) GO TO 8

304 $\star \star * \star *$ change resolution (smoothness) of ellipses $\star \star \star \star * *$ res $(1)=A I N(1)$. 75

res $(3)=.25^{*}$ res $(2)$

60 TO 8.20

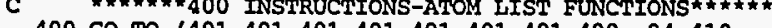

1
1 $\quad \begin{aligned} & 401,401,401,401,401,401,401,490,94,410 \\ & 401,401,401,401,401,94), N^{2}\end{aligned}$ 
401 CALI F400

GO TO 490

LO $420 \quad I=1,500$

$\operatorname{ATOMID}(I)=0$.

DTOMS ( $\mathrm{J}, \mathrm{I})=0$

490 IF (LATM) $8,8,491$

491 IF (NOUT.GE.0)

499 FORMAT (1HO, 10X, 23HCONTENTS OF ATOMS ARRAY/(15X, 10F10.0))

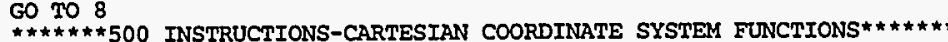

$500 * \hbar \pi \hbar * 2500$

IF (NOUT, GE 0 )

STRITE (NOUT, 503) (ORGN $(J), J=1,3$,

503 FORMAT(1H0,10X, 44HORIGIN FOR PROTECTION AXIS IN CRYSTAL COORD.

1,3 F15.6)

IF (NJ3 -3) 507, 539, 504

504 IF (NJ3-6) 601,5

SWRITE (NOUT, 529$)$

IF (NOUT, GE,

$\operatorname{QWRITE}(\operatorname{NOUT}, 519)((\operatorname{REFV}(J, I), I=1,3),(\operatorname{AAREV}(J, I), I=1,3), J$

$1=1,3)$

509 FORMAT (1HO10X, 49HORTHONORMAL WORKING VECTORS RASED ON CRYSTAL AXE 1S18X, 33HPOST-FACTOR TRANSFORMATION MATRIX/16X, 8HXX VECTOR8X, 8HY VEC 2TOR $8 X, 8 \mathrm{HZ}$ VECTOR)

19 FORMAT (1H 10X, 3E16.7,8X, 3E16,7)

529 FORMAT (1H010X, 51HORTHONORMAL REFERENCE VECTORS BASED ON CRYSTAL AX OR TRANSFORMATION MATRIX/16X, $8 \mathrm{HX}$ VECTOR8X, 8HY VE 2CTOR8X, 8HZ VECTOR)

539 IF (NOUT.GE.0)

TF (NOUT GE, (N)

EWRITE (NOUT, 519) ( (WRKV (J,I) ,I=1,3), (AAWRK $(J, I), I=1,3), J$ $1=1,3)$

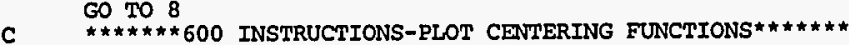
600 CALL F600

\&WRITE (NOUT, 609) XO(1), XO(2), SCAL1, SCAL2

609 FORMAT (1H010X, 31HORIGIN POINT IN PLOTTER COORD. (F6.2, $2 \mathrm{H}, \mathrm{F} 6.2,8 \mathrm{H})$ 1 IN. / $11 X, 15 \mathrm{HOVERALL}$ SCALE $=F 6.3,32 \mathrm{H}$ INCH/ANGSTROM ELLIPSOID SCA $2 \mathrm{LE}=\mathrm{F} 6.31$

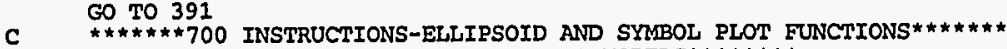
$\star * \star * \star * \star * *$ FILL OUT DETAILS FOR SPECIAL MODELS

$7006 \operatorname{AIN}(3)=1$.
$G 0$ TO 703

GO TO 703
$\operatorname{AIN}(3)=8$.

GO TO 703

$702 \operatorname{AIN}(3)=0$.

$03 \operatorname{AIN}(1)=4$.

$\operatorname{AIN}(2)=0$.

$\operatorname{AIN}(4)=0$.

$704 \operatorname{AIN}(1)=3$.
Go TO 706

$05 \operatorname{AIN}(1)=1$.

$706 \operatorname{AIN}(2)=0$

$709 \operatorname{AIN}(4)=5$

GO TO 8

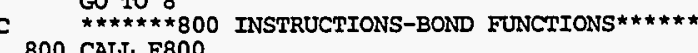

800 CALL F800

60 TO 8

900 CALL F900

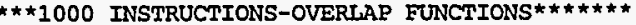

1000 CALL F1000

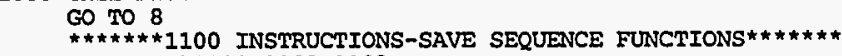

1100 IF (NJ2-2) 1101,1102,1103

GO TO 1104

1102 ISAVE $=0$

CCC $\quad \mathrm{END}$

END FILE NSR

GO TO 1104

1103 ISAVE $=-1$

1104 RTIND

3000 CALL EXITNG (NG)

EUND

C ARCCOS $(x)$ IN DEGREES

$\mathrm{X}=\operatorname{SIGN}(1.0, \mathrm{X})$

$1 \mathrm{X}=\operatorname{SIGN}(1.0, \mathrm{X})$

3 ARCCOS $=180.0+$ ATAN $(S Q R T(1.0-X \star X) / X) \star 57.29577951$

GO TO 6

4 ARCCOS $=90.0$

GO TO 6

ARCCOS=ATAN $(\operatorname{SQRT}(1.0-X * X) / X) * 57.29577951$

RETURN

ENDROUTINE ATOM (QA, $Z$ )

C ATOM COORDTNATE SUBROUTINE

$R E A L \star 8$ QA, TA, D100K

DIMENSION $X(3), Z(3)$

REAL* 8 AIN, ATOMID

CHARACTER 44 TITLE, TITLE2

CHARACTER $* 8$ CHEM

, A

(500), ATOMS $(3,500), \mathrm{BB}(3,3), \mathrm{BRDR}, \mathrm{CD}(8,20)$

CONT (5), D $(3,130), D A(3,3), D P(2,130)$, DISP, EDGE, FORE, ES $(3,3,96)$

, IN, ITILT, KD $(5,20)$, LATM, NATOM, NCD, NJ, NJ2, NOUT, NSR, NSYM

, SCAL2 SCL, SYMB $(3,3)$, TAPER, THETA, TITLE (18), TITLE2 (18), TS $(3,96)$

, VIEW VT (3, 4) V1 (4), V2 (3),V3 (3), V4 (3), V5 (3), V6 (3), WRKV (3,3)

, XING $(3)$, XÓ(3), XT(3)

INTEGER *2 IDENT

源

1, IDENT $(2,505)$, MAXATM

$\mathrm{K}=0 \mathrm{~A} / \mathrm{D} 100 \mathrm{~K}$ 
$\operatorname{IF}(\mathrm{K}) 109,109,117$

$X(1)=0.0$

$x(2)=0.0$

$x(3)=0.0$

117 IF(K-NATOM) $119,119,503$

503 NG=5

$$
\text { GO TO } 325
$$

$119 \mathrm{DO} 123 \mathrm{~J}=1$,

$123 \quad X(J)=P(J, K)$
125 TA $=D A B S(Q A)$

KSYM=DMOD (TA, D100K)

$\mathrm{Kr}=\mathrm{KSYM} / 100$

IF (KS-NSYM) 203,203,403

$\mathrm{NG}=4$

GO TO 325
203 IF (KS) 403,205, 213

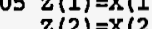

$\mathrm{z}(3)=\mathrm{x}(3)$

213 DO $223 \mathrm{~K}=1$,

$\mathrm{Z}(\mathrm{K})=\mathrm{TS}(\mathrm{K}, \mathrm{KS})$
$\mathrm{DO} 223 \mathrm{~J}=1,3$

$\mathrm{D} 223 \mathrm{~J}=1,3$
$\mathrm{Z}(\mathrm{K})=\mathrm{Z}(\mathrm{K})+\mathrm{FS}(\mathrm{J}, \mathrm{K}, \mathrm{KS}) * \mathrm{X}(\mathrm{J})$

$311 \mathrm{IF}(\mathrm{KT}) 403,325,313$

313 IF (KT

GO TO 325

$17 \mathrm{K1}=\mathrm{KT} / 100$

$\mathrm{K}=\mathrm{KT}-100^{\star} \mathrm{K}$

$\mathrm{K} 2=\mathrm{K} / 10$
$\mathrm{~K} 3=\mathrm{K}-10^{*} \mathrm{~K}$

$K(1)=Z(1)+F L O A T$
$Z(K 1-5)$

$Z(2)=Z(2)+$ FLOAT $(K 2-5)$

25 RETURN

SUBROUTINE AXEQB (AI, X, B1, JJJ)

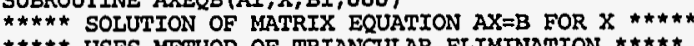

C $* \star \star \star *$ B AND $X$ HAVE DIMENSIONS (3,JJJ), A IS ALWAYS $(3,3)$

***** TO INVERT A MAKE B 3 BY 3 IDJJ),

DIMENSTON $A 1(3,3), A(3,3), B(3,3), B 1(3,3), X(3,3)$

C NV=JJJ

DO 2 I=1,3

IF (NV-J) $2,1,1$

$1 B(I, J)=B 1(I, J)$

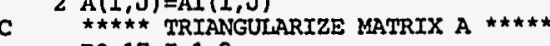

Do $17 \mathrm{I}=1,2$

$S=0.0$

$R=A B S(A)(J, I)$
IF $(R-S) 4,3,3$

$3 \quad S=R$

$4 \stackrel{L}{\mathrm{C}=\mathrm{J}}$
$\operatorname{IF}(L-I) 5,10,5$

$D O 6$ S $J=I, 3$

$S=A(I, J)$
$A(I, J)=A(I, J)$

$A(L, J)=S$
$A(I, U)$

$S=B(I, J)$

$B(I, J)=B(I, J)$

$8 \quad B(L, J)=S$

IF (TEM) $11,17,11$

$11 \mathrm{TPO}=\mathrm{T}+1$

DO $16 \mathrm{~J}=\mathrm{IPO}, 3$

$\operatorname{IF}(A(\mathrm{~J}, I)) 12,16,12$

$12 \mathrm{~S}=\mathrm{A}(\mathrm{J}, \mathrm{I}) / \mathrm{TEM}$

$A(J, I)=0.0$

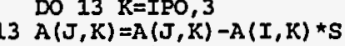

$15 B(J, K)=B(J, K)-B(I, K) * S$

16 CONTINUE

17 CONTINUE DO $20 \mathrm{I}=1,3$

I $=\operatorname{AMAX} 1(1, E-25, \operatorname{AMaX} 1(A(1,1), A(2,2), A(3,3)) * 1, E-15)$

DO $24 \quad K=1, N$

$N=4-I$
$M=N+1$

$\mathrm{TEM}=\mathrm{B}(\mathrm{N}, \mathrm{K})$

$\operatorname{IF}(3-M) 23,21,21$

DO $22 \mathrm{~J}=\mathrm{M}, 3$

22 TEM $=T E M-A(N, J) * B(J, K)$

$\mathrm{B}(\mathrm{N}, \mathrm{K})=\mathrm{TEM} / \mathrm{A}(\mathrm{N}, \mathrm{N})$

$24 \mathrm{X}(\mathrm{N}, \mathrm{K})=\mathrm{B}(\mathrm{N}, \mathrm{K})$

RETURN

SUBROUTINE AXES $(U, V, X$, ITTYPE)

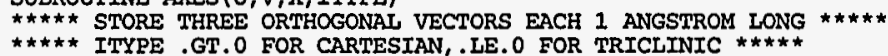

$* * * * *$ IABS (ITYPE) $=1$ W(1) $=0, W(2)=(U X V), W(3)=\operatorname{UX}($ UXV) $* * * * * *$

$\star \star \star \star \star$ ITYPE $=0$ W(1) $=A, W(2)=(A X B) X A, W(3)=(A X B), A B C=C E L L$ VECTORS $* \star *$ DIMENSION $U(3), V(3), W(3,3), X(3,3)$

IF(T) 115,10

$U(2)=0$

$U(3)=0$.

$V(1)=0$.

$V(2)=1$.

$115 \mathrm{DO} 125 \mathrm{~J}=1,3$

IF(IABS (IT) - 1) 145,135,145

35 CALL NORM(U, $V, W(1,2), I T)$

CALL NORM(U, $W(1,2), W(1,3), I T)$

$145 \operatorname{CALL} \operatorname{NORM}(U, V, W(1,3), I T)$ 
155 DO 195 I=1,3

195 CALI UNITY $(W(1, I), X(1, I), I C)$

RETURN

END

SUBROUTINE BOND (Z1, $\mathrm{Z2}, \mathrm{NB}, \mathrm{NA1}, \mathrm{NA} 2)$

REAL *8 $\mathrm{Z1}, \mathrm{Z2}$, WD (2), TD, D100,D1000,D100

$U(3,3), \operatorname{VUE}(3)$

DIMENSION V7 (3),W(13,2),z(3), RESB (2)

*8 ATN, ATOMID

CHARACTER*4 TITLE, TITLE2

COMMON NG, A (9), $\operatorname{AA}(3,3), \operatorname{AAREV}(3,3), \operatorname{AAWRK}(3,3), \operatorname{AID}(3,3)$

1 , AIN (140), ATOMIDD (500), ATOMS $(3,500), \mathrm{BB}(3,3), \mathrm{BRDR}, \mathrm{CD}(8,20)$

2 'CN (5), D (3,

4 , ORGN $(3), \operatorname{PAC}(3,5), \operatorname{PAT}(3,3), Q(3,3), \operatorname{REFV}(3,3), \operatorname{RES}(4), \operatorname{RMS}(5), \operatorname{SCAL} 1$

5 , SCAL2, SCL, SXMB $(3,3)$, TAPER, THETA, TITLE (18), TITLE2 (18), TS $(3,96)$

6 , VIEW, VT $(3,4), \mathrm{V} 1(4), \mathrm{V} 2(3$

, XING (3), XO (3), XT (3)

INTEGER $2^{2}$ IDEN

(3)

DATA RESB $/ .2, .08$

D100 $=100$.

D1000 $=1000$.

$\mathrm{D} 100 \mathrm{~K}=100$

NO $105 \mathrm{~J}=1,26$

$105 \mathrm{~W}(\mathrm{~J}, 1)=0$

$W D(1)=21$

WD $(2)=Z 2$

Do $135 \quad I=1,2$

CALL XXZ (WD(I), W(4,I),2)

10 DO

$\begin{array}{ll}110 & D O 115 \quad J=1,3 \\ 115 & W(J+6, I)=X T(J)\end{array}$

$K=W D(I) / D 100 K$

$\mathrm{L} 1=\mathrm{DMOD}(\mathrm{WD}(\mathrm{I}), \mathrm{D} 100)$

$\operatorname{CALL} \operatorname{PLTXX}(W(4, I), W(2, I))$

$120 \mathrm{NG}=10$

IF (NOUT.GE.0)

NF (NOUT.GE. 0)

CALL ERPNT' (WD (I), 800)

GO TO 134

128 IF (NJ2-10) 130, 134, 13

30 IF (NOUT.GE. )

作

134 continue

136 FORMAT (1H 10X, A6, 3H (I3,1H, I3, I2, 4H) 2F8.2, 5X,3F8.3,13X, 3F8.4) IF (NG1) $999,137,999$

$137 \operatorname{CALI} \operatorname{DIFV}(W(7,1), W(7,2), V 7)$
DIST $=S R R T$ (VMV (V7, AA, V7)

143

C $\star \star \star \star \star$ LINE BONDS AND CENTERED SYMBOLS $(803,813)$

HGT $=S C L * .12$

c *** $\operatorname{CRTEP} \operatorname{CIMBOL}(\mathrm{W}(2,1), W(3,1), \mathrm{HGT}, \operatorname{MOD}(\operatorname{IDINT}(W D(1) / D 100 K), 10), 0 .$, CALL SIMBOL $(W(2,2), W(3,2), \operatorname{HGT}, \operatorname{MOD}(\operatorname{IDINT}(W D(2) / D 100 K), 10), 0, .-$ c 12)

(*) is available in ORTEP-III.

$c * \star *$ It is triggered by the negative value for argument 6 .

ent 4 is ignored by SIMBOL.

CALL SIMBOL $\left(W(2,1), W(3,1), H_{G T}, \cdots, 0,-1\right)$

GO TO 570

C $\star * \star * \star$ STICK BONDS FOR $801,802,811,812 * \star \star \star \star *$

IF (KODE) $145,144,146$

144 NBND=0

$\mathrm{GO} T \mathrm{TO} 148$
$\mathrm{KODE}=-\mathrm{KODE}$

$146 \mathrm{NBND}=128 / 2$ * $\mathrm{KODE}$

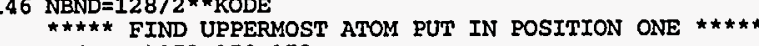

$W(12,2)=1$

$\operatorname{IF}(W(6,1)-W(6,2)) 165,175,175$

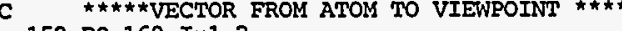

$\begin{array}{ll}152 \text { DO } & 160 \\ \text { DO } & 155 \quad \mathrm{~J}=1,2 \\ \end{array}$

$155 \mathrm{~W}(\mathrm{~J}, \mathrm{I})=-W(\mathrm{~J}-6, \mathrm{I})$

$155 W(12, I)=W(22, I)+V I E W$

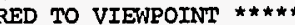

W(13,I) $=\mathrm{WV}(W(10, I) ; W(10, I))$

C ${ }_{165} \mathrm{DO} 170 \mathrm{~J}=1,13$

$W(J, 1)=W(J, 2)$

$170 \mathrm{~W}(\mathrm{~J}, 2)=\mathrm{TI}$

$W D(1)=W D(2)$

$W D(2)=T D$

C 175 DO*

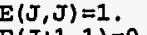

$180 \mathrm{E}(\mathrm{J}+5,1)=0$

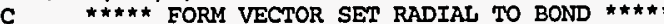

CALL DIFV(W(4,2), W(4,1), DA $(1,3))$

CALL UNITY (DA $(1,3)$, V3,1) DO $183 \quad I=1,3$ V2 $(x)=0.0$

$181 \mathrm{~V} 2(I)=\mathrm{V} 2(I)+\operatorname{AAREV}(J, 3) * \operatorname{WRKV}(J, I)$

IF (VIEW) $183,183,182$

$182 \mathrm{~V} 2(\mathrm{I})=\mathrm{V} 2(I) * \mathrm{VIEW}-0.5^{*}(\mathrm{~W}(I+3,1)+W(I+3,2)$

83 CONTINUE

CALL UNITY (V2, V2,1) 


\section{$\mathrm{T} 6=\mathrm{ABS}(\mathrm{VV}(\mathrm{V} 3, \mathrm{~V} 2))$}

C $\star \star \star \star \star$ ALTERNATE CAIC IF BOND IS ALONG REFERENCE VIEW DIRECTION $\star \star \star$ 185 DO $186 \quad J=1,3$

$\mathrm{V} 2(\mathrm{~J})=W(\mathrm{~J}+9,1)+W(\mathrm{~J}+9,2)$

CALL UNITY (V2, V2, 1$)$

TF (. 9994-T6) 390,390,187

187 CALL AXES (V3, V2, B1,1)

作 $190 \mathrm{~J}=1,3$

$D A(J, 1)=-B 1(J, 2) * T 1$

$90 \mathrm{DA}(\mathrm{J}, 2)=-\mathrm{B} 1(\mathrm{~J}, 3) \star \mathrm{T} 1$

IF (NBND) $500,500,195$

*

$195 \mathrm{~T} 1=\mathrm{CD}(3, \mathrm{NB}) * \mathrm{SCL}$

$$
\text { NRESOL }=4
$$

DO $200 \mathrm{~J}=1,2$
IF (TI.GE.RESB(J)) GO TO 202

IF (NBND. LE.NRESOL) GO TO 202

200 NRESOL $=$ NRESOL

C CALL RADIAL (NBIS)

DO $380 \mathrm{III}=1,2$

CALL PAXES (WD (II) , 2 )

IF (NG) $205,210,205$

205 CALI ERPN

GO TO 999 DOES BOND GO TO

C $210 \mathrm{~T} 1=3-\mathrm{II} * 2$

$D 212 \mathrm{~J}=1,3$

$212 \operatorname{VUE}(\mathrm{J})=0$.

$215 \operatorname{IF}(\mathrm{VMV}(\mathrm{V} 3, Q, \mathrm{~W}(10, \mathrm{II}))) 220,260,260$

IF (VIEW) $240,240,225$

C IF $* \star \star \star$ DERIVE TANGENT CONE DIRECTLY WITHOUT ROTATING COORDINATES ** T2 $=-(\operatorname{SCAL} 2 * \operatorname{RMS}(1) * \mathrm{RMS}(2) * \operatorname{RMS}(3)) * \star 2$

DO $230 \mathrm{~J}=1,3$

V1 (J) $=-W(J+9$, II $) / S C A L$

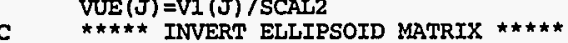

DO $230 \mathrm{~K}=\mathrm{J}, 3$

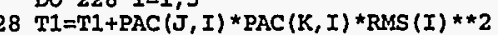

$\mathrm{U}(\mathrm{J}, \mathrm{K})=\mathrm{TI}$

$230 \underset{\star \star \star \pi, J \hbar}{U(K, J)=T}$

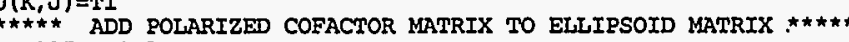

DO $235 \mathrm{~J}=1,3$

$V J 1=V 1(J i)$

$\mathrm{J} 2=\mathrm{MOD}(\mathrm{J}+1,3)+1$

VJ2 $=\mathrm{V} 1$ ( $\mathrm{J} 2$ )

DO $235 \mathrm{~K}=\mathrm{J}, 3$

$\mathrm{K} 1=\mathrm{MOD}(\mathrm{K}, 3)+1$
$\mathrm{S}(\mathrm{J}, \mathrm{K})=\mathrm{T} 2 * \mathrm{Q}(\mathrm{J}, \mathrm{K})+(\mathrm{VJ} 2 *(\mathrm{U}(\mathrm{J} 1, \mathrm{~K} 1) * \mathrm{~V} 1(\mathrm{~K} 2)-\mathrm{U}(\mathrm{JI}, \mathrm{K} 2) * \mathrm{VI}(\mathrm{K} 1))$

$235 S(K, J)=S(J, K)$

$T 5=0.0$

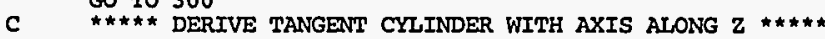

$240 \mathrm{~T} 1=-1.0 / \mathrm{Q}(3,3)$

DO $250 \quad J=1,2$

$245 S(K, J)=0(K, J)+Q(K, 3) * Q(J, 3) * T 1$

$250 S(J, J)=0.0$

$S(3,3)=0.0$

GO TO 270

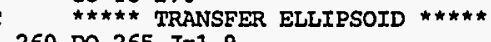

260 DO $265 \mathrm{~J}=1,9$

IBND $=I I$

270 T5 $=1$.

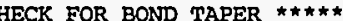

$300 \mathrm{IF}$ (II-2) 305, 310,310

305 RADIUS $=1 .+T 6 *$ IAPER

RO 120

320 CALL MV $(S, V 3, V 4)$

C T2 $=$ VV $(\mathrm{V} 3, \mathrm{~V} 4)$ V

$\mathrm{KL}=5-\mathrm{II}-\mathrm{II}$

IF (NJ2-21) 324, 322, 322

322 KSTP $=32$

DO $335 \mathrm{~K}=1,65, \mathrm{KSTP}$

(J) $*$ RADIUS

25 V5 $(J)=$ V $6(\mathrm{~J})+\mathrm{VUE}(\mathrm{J})$

$\mathrm{T} 3=\mathrm{VV}(\mathrm{V} 5, \mathrm{~V} 4)$

$T 4=T 3 * T 3-T 2 *(V M V(V 5, S, V 5)-T 5)$

330 IF (T4) $345,330,330$

$\mathrm{T} 1=(\mathrm{T} 4-\mathrm{T} 3) / \mathrm{T} 2$

$\mathrm{T} 1=(\mathrm{T} 4-\mathrm{T} 3) / \mathrm{T} 2$
$\mathrm{~T} 3=(-\mathrm{T} 4-\mathrm{T} 3) / \mathrm{T} 2$

$\mathrm{L}=\mathrm{K}+\mathrm{KL}-1$

DO $335 \mathrm{~J}=1,3$

D $(J, L)=(\mathrm{V} 6(\mathrm{~J})+\mathrm{T} 1 * \mathrm{~V} 3(\mathrm{~J}))$ *SCL

$\mathrm{D}(\mathrm{J}, \mathrm{L}+1)=(-\mathrm{V} 6(\mathrm{~J})-\mathrm{T} 3 * \mathrm{~V} 3(\mathrm{~J})) * \mathrm{SC}$

IF (IBND+21-NJ2) $360,338,360$

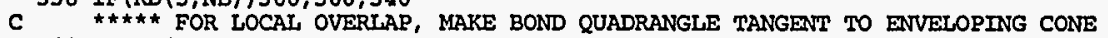
T3=VW(VUE, V4)

$\mathrm{T} 4=\mathrm{T} 3 \star \star 2-\mathrm{T} 2 *(\mathrm{VMV}$ (VUSE, S, VUE) $-\mathrm{T} 5)$

345 NG=13

CALL ERPNT (WD (II) , 800

$350 \mathrm{~T} 1=($ SQRT $(\mathrm{T} 4)-\mathrm{T} 3) / \mathrm{T} 2$

$\mathrm{TA}=(\mathrm{T} 1 * \mathrm{~V} 3(J) * \mathrm{SCL}-0.5 *(\mathrm{D}(\mathrm{J}, \mathrm{KL})+\mathrm{D}(\mathrm{J}, \mathrm{KL}+64))) * 1.001$

$\mathrm{D}(\mathrm{J}, \mathrm{KL})=\mathrm{D}(\mathrm{J}, \mathrm{KL})+\mathrm{T} 4$

$355 \mathrm{D}(\mathrm{J}, \mathrm{KL}+64)=\mathrm{D}(\mathrm{J}, \mathrm{KL}+64)+\mathrm{T} 4$

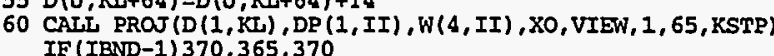


365 CALL PROT (D $(1, K L+K S T P+1), D P(1, I I+64+K S T P), W(4, I I), X O, V I E W, 1$, 65-KSTP, KSTP

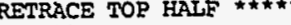

(II-1)*KSTP

KTP, KK, KSTP

$\mathrm{L}=\mathrm{K}+\mathrm{II}$

$M=L+64$

$375 \operatorname{DP}(1, M)=D P(1, N)$

380 CONTINUE

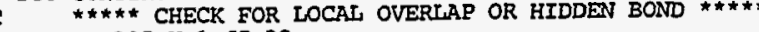
DO $395 \mathrm{~K}=1,65,32$

$T 1=0$.

DO $385 \mathrm{~J}=1,2$

$T 1=T 1+(D P(J, K)-W(J+1,1)) \star \star \star 2$

$385 \mathrm{~T} 2=\mathrm{T} 2+(\mathrm{DP}(\mathrm{J}, \mathrm{K}+1)-\mathrm{W}(\mathrm{J}+1,1)) * * 2$

IF (T2-T1) $390,390,395$

$C^{395 \underset{* \star * \star *}{\text { CONTINUE }} \text { CALI GLOBAL OVERLAP ROUTINE } * \star * \star \star *}$ $I C Q=0$

CALI LAPBOO (NA1, NA2, ICO)

IF (NJ2-21) $400,999,999$

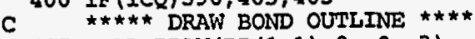

405 CALL DRAW (DP $(1,1), 0,0,3)$

DO $415 \mathrm{~K}=$ NRES1, 129 ,NRESOL

415 CALU DRAN(DS1, $(1, x), 0 ., 0 ., 21$

CALL DRAW (DP $(1, K), 0,0,2)$

CALI DRAW (DP $(1,65), 0,0,2)$

$25 \mathrm{~K}=65$

$30 \mathrm{~K}=\mathrm{K}-\mathrm{NBND}$

IF (X-1) $500,500,435$

CALI DRAW (DP (1, $K, 1), 0 ., 3\}$ $\mathrm{K}=\mathrm{K}-\mathrm{NBND}$

440 CALL DRAW $(\mathrm{DP}(1, \mathrm{~K}+1), 0,0,3)$

CALL DRAW (DP $(1, K), 0,, 0,2)$

GO TO 430

$O F F=C D(5, N B)$

IF (HGT) $570,570,510$

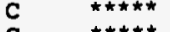

$510 \quad K=0 \quad \operatorname{DIFV}(W(7,2), W(7,1), V 7)$

CALL VM (V7, AAREV, V1)

CAII AXES (V1, $E(1,3), U, 1)$

DO $535 \quad I=1,3$

$T 1=1$

IF (I-2) 515, 515, 520

$515 \operatorname{IF}(\mathrm{VV}(U(1, I), \operatorname{SYMB}(1, I))) 525,530,530$

$K=K+1$

$530 \quad \mathrm{DO} 535 \mathrm{~J}=1,3$
$U(J, I)=U(J, I) * T 1$

$\operatorname{VT}(J, I)=B 1(J, I)$

540 VT $(J, 4)=.5^{\star}$ (W

**** CHECK FOR EXCESS FORESHORTENING ****

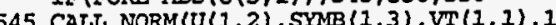

$\operatorname{VT}(1,3)=\operatorname{SYMB}(1,3$

$\operatorname{VT}(2,3)=\operatorname{STMB}(2,3)$

$\mathrm{HGT}=\mathrm{CD}(6, \mathrm{NB})$

$\mathrm{OFF}=\mathrm{CD}(7, \mathrm{NB})$

IF(

(1) $=\mathrm{VT}(1,4)-\mathrm{HGT}(11,+3, * \mathrm{~T} 1) / 7$

(1) $=\operatorname{VT}(2,4)+O F F-H G T * 5$

$z(3)=\operatorname{VT}(3,4)$

ITILT $=1$

$\mathrm{T} 9=10, \star \star I 9$

(1)

570 ITILT $=0$

IF (NJ2-10) 580,999,999

IF (NOC.Ge.

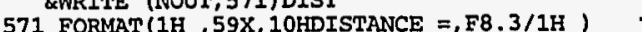

GO TO 999

CALL ERPNT (WD (2), 800)

999 RETURN

SUBROUTINE DIFV $(X, Y, Z)$

C $\quad$ VECTOR - VECTOR

DIMENSION $X(3), Y(3), Z(3)$

$Z(1)=X(1)-Y(1)$

$Z(2)=X(2)-Y(2)$
$Z(3)=X(3)-Y(3)$

$z(3)=X(3)$
RETURN

SUBROUTINE DRAW (W, DX, DY, NPEN)

REAL *8 AIN ATOMID

CHARACTER $\star 4$ TITLE, TITLE2

COMMON NG, A $(9), \operatorname{AA}(3,3), \operatorname{AAREV}(3,3), \operatorname{AANRK}(3,3), \operatorname{AID}(3,3)$

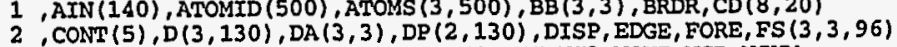

3 . IN, ITILT, KD $(5,20)$, LATM, NATOM, NCD, NJ, NJ2, NOUT, NSR, NSMM

4 ,ORGN $(3), \operatorname{PAC}(3,5), \operatorname{PAT}(3,3), Q(3,3), \operatorname{REFV}(3,3), \operatorname{RES}(4), \operatorname{RMS}(5), \operatorname{SCAL} 1$

5 , SCAL2, SCL, SYMB $(3,3)$, TAPER, THETA, TITLE (18), TITLE2 (18), TS $(3,96)$

6 , VIEN, VT $(3,4), \operatorname{V1}(4), \operatorname{V2}(3), \mathrm{V} 3(3), \mathrm{V} 4(3), \mathrm{V} 5(3), \mathrm{V} 6(3), \mathrm{WRKV}(3,3)$

,XING (3), XO(3),XT(3)

$Y(1)=W(1)+D X$

IF (ITILT) 115, 140,115

C 115 Y

DO 120 I=1,3

$120 Z(I)=Y(I)-V T(I, 4)$ 
DO $130 \quad I=1,3$

$130 x(1)=V T(I, 1) \star Z(1)+V T(I, 2) \star Z(2)+V T(I, 3) \star Z(3)+V T(I, 4)$

cati

IF $160 \quad J=1,2$

$150 \operatorname{IF}(Y(J)-.1) 155,160,160$

$155 \mathrm{Y}(\mathrm{J})=.1$

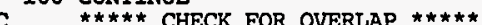

\section{CALL LAPDRW (Y, NPEN, NCO)}

IF (NCQ) $165,165,170$

C ${ }^{* * * * \star}$ CALI PLOTTING ROUTINE IF NO OVERLAPPING ELEMENTS ARE STORED 165 CALL SCRIBE (Y, NPEN)

170 RETU

\section{SUBROUTINE EDITR}

SUBR

CHARACTER $* 8$ CHEN

COMMON NG, A $(9), \operatorname{AA}(3,3), \operatorname{AAREV}(3,3), \operatorname{AANRK}(3,3), \operatorname{AID}(3,3)$

$1, \operatorname{AIN}(140), \operatorname{ATOMID}(500), \operatorname{ATOMS}(3,500), \mathrm{BB}(3,3), \operatorname{BRDR}, \mathrm{CD}(8,20)$

, $\operatorname{CONT}(5), \mathrm{D}(3,130), \mathrm{DA}(3,3), \mathrm{DP}(2,130), \mathrm{DISP}, \mathrm{EDGE}, \mathrm{FORE}, \mathrm{FS}(3,3,96)$

4 , ORGN (3), PAC $(3,5), \operatorname{PAT}(3,3), Q(3,3), \operatorname{REFV}(3,3), \operatorname{RES}(4), \operatorname{RMS}(5), \operatorname{SCAL} 1$

5 , SCAL2, SCL, SMMB $(3,3), \operatorname{TAPER}, \operatorname{THETA}, \operatorname{TITLE}(18), \operatorname{TITLE} 2(18), \operatorname{TS}(3,96)$

, VIEW, VT $(3,4), \operatorname{V1}(4), \operatorname{V2}(3), \mathrm{V} 3(3), \mathrm{V} 4(3), \mathrm{V} 5(3), \mathrm{V} 6(3), \operatorname{VRKV}(3,3)$ , XING (3), XO (3), XTT (3)

INTEGER $2_{2}$ IDENT

COMDA

com

, INQ, OUE (96), hque (96)

CHARACTAR*73 npe, ndraw, norient, nva

character $\star 80$ answer, card

CHARACTER $\star 1$ CH

$11111111112222222222333333333334444444445555^{\prime}$, 111111111122222222233333333334444444444555',
*'55555556666666666777', ', 1X,'LINE', 1x,'1234567890123456789012345',
*'67890123456789012345678901234567890123456789012')

NUM=1

PRINT PART OR ALL OF COMMAND QUE ***

NUM1=MAXO $(1, N U M-2)$

100 WRITE $(*, 103)$

FORMAT (1X)

(, OUE (J), $J=$ NOM1, NQUE)

111 FORMAT (1X, I3,2X,A72)

115 DISPLAY PRO $(*, 121)$

121 FORMAT $\left(/{ }^{\circ}, \mathrm{C}=\mathrm{C}\right.$ ange 1 ine

\&) \# [\#] $, 1,1$, I=Insert line before \#

\&) $[\#][\#]$

modified instruction set

$\&, 1, \cdot$ P=Save drawing as Postscript

Delete line $(s$
$T=$ Type line(s

one arawing a

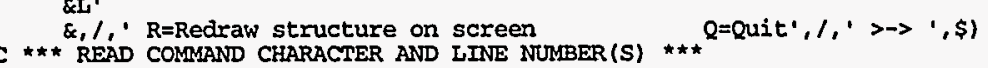

131 format (a) if (answer (1:1) .eq. ' ') go to 133

If (answer (1:1).ge. ' 1 ' . and. answer(1:1).1e.'9') go to 133

last $=1$ end (answer)

132 i=2, 1 sest

if ( ch.ge. ' $a$ ' and.ch.le. ' $z$ ') or.

' (ch.ge. 'A' and.ch.le. ' $Z$ ')) go to 133

$i c h=i$ char $(\mathrm{ch})-48$

132 continue

go to 135

134 format $(/, \cdots * \star$ INVALID INPUT! Enter 1 letter and 0,1 , or 2 integer "s separated by spaces. $\star \star \star 1$ )

go to 115

135 answer=answer (1: 1ast) // 00

read (answer, $*$ ) ch, num, num2

write $(15,136)$ answer $(1: 1)$, answer $(2: 75)$

136 format $(., 13$, a a $\cdots, \cdot 1,1 x, a)$

rewind (15)

read $(15, *) \mathrm{ch}$, num, num2

close (15)

$\mathrm{NUM}=\mathrm{MAXO}(1, \mathrm{NOM})$

if (num2. gt.nque) then

137 format $(1,1 \star \star \star$ value out of range $\star \star \star 1)$ go to 115

end if

IF (NUMA. GT. NUM2) NUM2=NU1

write $6, *)$ '

(d') GO TO 210

IF (CH.EQ.' 'D'.or.ch.eq. 'd') GO TO 240

IF (CH.EQ. ' $C$ '.or.ch. eq. ' $c$ ') GO TO 270

IF (CH.EQ. 'I'.Or.ch.eq. ' $i$ ') GO TO 310

IF (CH.EQ.' 'S'Or. Ch. eq. ' $O$ ') GO TO 410

IF (CH.EQ. 'R'. or.ch.eq. ' $r$ ') GO TO 54

IF CH. ' 2 'or.ch.eq.' ' ' ' GO To 590

IF (CH.EQ.' 'H'. Or.ch.eq. ' $h$ ') GO TO 520

GO TO 115

$* \star \star$
210 if if (numz. eq. 0 ) num2=nque

if (numz, eg,

URTTE $\left(*^{*}, 111\right)$ (J, OUE (J) J J=NUM, NUM2)

GO TO 115

(numz.eq.0) then supply line number(s) with command $\star \star \star$.

go to 115

end if

$I=N U M, N U M 2$

NQUE $=$ NQUE-1

DO $250 \quad J=N U M, N O U E$

250 QUE $(J)=\operatorname{QUE}(\mathrm{J}+1)$

$260 \mathrm{QUE}(\mathrm{NQUE}+1)$

$C *$ * GO TO 100 
270 if (numz.eq.0) then write $(6, *)$
go to 115$$
\text { end if }
$$

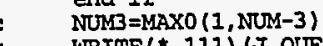

WRITE $(*, 111)$ (J, OUE (J), J=NUN3, NUM)

271 format $\left(i^{27 \pi \star}\right.$ NOTE: Type a to substitute a space in the original li cne. $\star * * 1,1$

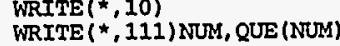

WRITE $(*, 276)$ NUI

276 FORMAT (iX, I3, ', \$)

read $(*, 281)$ tline

281 FORMAT (A72)

do $282 \quad i=1,72$

if (tline (i:i).ne.' ") then

.eq. 'Q') then

que (num) $(i: i)=$

$$
\text { que }
$$

282 continue

write $(*, 283)$

tine now reads:

RRITE $(*, 284)$ QUE (NUM)

284 format $(6 x, a)$

285 format $(/, \cdot$ Hit ENTER or RETURN key $'$, $\$$ read $(*, 131) \mathrm{CH}$

* $* *$ INSERT A NEW LINE ***

write $(6, *), * * \star$ Supply line number with command $* * *$ 1 go to 115

IF (NQUE+1 GT. 96) GO TO 115

NQUE $=$ NQUE +1

$\mathrm{NN}=\mathrm{NQUE}$

$N=$ NQUE-NUM

QUE (NN) $=$ QUE (NN -1

$320 \mathrm{NN}=\mathrm{NN}-1$

MUMA $=M A \times O(1, N U M-$

NUM1 $=\operatorname{MAXO}(1, N U M-1)$

write $(*, 10)$

11) (J, QUE (J) , J=NOMA , NOM1)

WRITE $(*, 276)$ NUM

NEXT $=$ NEXT +1

$C * \star$ RETRIEVE OID SET OF INSTRUCTIONS ***

410 DO $415 \mathrm{~J}=1$, NBACK

15 que $(j)=$ hque $(j)$

NUM=1

NUM1 $=1$

NQUE $=$ NBACK
NUM $2=$ NOUE

GO TO 100

c *** SAVE CURRENT SET OF INSTRUCTIONS ***
420 CONTINUE

421 write $(*, 422)$

22 format ( Enter file name: $\quad, \$$

read $(\star, 131)$ answer

rewind (NED)

read (NED, 131, end $=440)$ card

write (16,131) card(1: iend(card))

40 WRITE $(16,450)$ ( QUE (I) , I=1, NQUE)

450 FORMAT(A73)

close (16)
GO TO 115

460 write $(6, *)$ 'File already exists. Choose a different name.'

*** SO to 421 SAVE PICTURE AS POSTSCRIPT***

510 ndraw $=2$

go to 541

PICTURE AS HPGL***

520 ndraw $=3$

*** REDRAW PICTURE ***

540 ndraw $=1$

(1) save

541 open (16, file= 'TEP. NEW' , status= ' unknown')

read (NED, 131, end $=543$ ) card write $(16,131)$ card(1:iend(card))

go to 542
WRITE $(16,450)$ (QUE (I), I=1, NQUE)

call recycle

C SUBROUTINE EIGEN (W, VALU, VECT)

DIMENSION $W(3,3), \operatorname{VALU}(3), \operatorname{VECT}(3,3), A(3,3), B(3,3), V(3), U(3)$

C $\star * \star * *$ STATEMENT FUNCTION $\star * \star \star \star *$

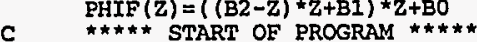

ERRND $=5 . E-7$

SIGMA $=0$.

Do $115 \quad J=1,3$

TE $115=W(I, J)$

$A(I, J)=T E M$

115 SIGM

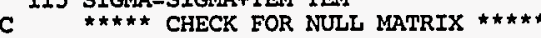
IF (SIGMA) $230,230,120$

120 SIGMA $=$ SQRT (SIGMA)

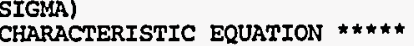

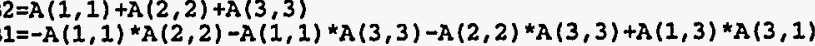
$B O=A(1,1) * A(2,2) * A(3,3)+A(1,2) * A(2,3) * A(3,1)+A(1,3) * A(3,2) * A(2,1)-$ $1 A(1,3) \star A(3,1) * A(2,2)-A(1,1) \star A(2,3) * A(3,2)-A(1,2) \star_{A}(2,1) \star A(3,3)$ $\mathrm{X}=0$.

TEMM=PHIF (SIGMA) 
VNEW $=0.0$

140 IF (TEM) $140,140,165$

GO TO 165

$145 Y=0$.

TF (TEM) $165,165,150$

$150 \mathrm{X}=-\mathrm{x}$

C

65 VNEW $=(X+Y) * .5$

75 IF (PHIF (VNEW) ) 180,250,185

$180 \mathrm{X}=\mathrm{VNEW}$

GO TO 200

$185 \mathrm{Y}=\mathrm{VNEW}$

VOLD $=$ VNEW
VNEW $=(X+Y) *$

TEM=ABS (VOLD-VNEW)

IF (TEM-ERRND) 250, 250, 205

$210 \mathrm{IF}$ (ABS (TEM/VOLD) -ERRND) 250,250,225

225 CONTINUE

C $\star \star \star \star \star \star$ DID

230 NG $=6$
GO TO 400

C $\star \star \star \star \star \star$ STORE FIRST ROOT $\star \star \star \star \star *$

C 250 U(3)=VNEW

$* \star \star \star \star *$
$\mathrm{C}=\mathrm{B} 2-\mathrm{VEFLA}$

$\mathrm{C} 0=\mathrm{B} 1+\mathrm{C} 1 * \mathrm{VNEW}$

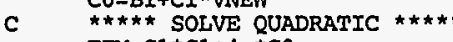

$T E M=C 1 * C 1+4,{ }^{*} \mathrm{CO} 0$

C $* \star \star \star \star$ IGNORE IMAGINARY COMPONENT OF COMPLEX ROOT $\star * \star * \star$ 5 TEM $=0$. GO TO 265

260 TEM $=S Q R T$ (TEM)
$265 \mathrm{U}(1)=.5^{\star}$ (C1-TEM)

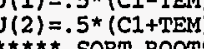

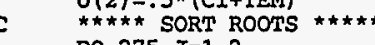

DO $275 J=1,2$

$\mathrm{U}(\mathrm{J})=\mathrm{U}(3)$

$\mathrm{U}(\mathrm{J})=\mathrm{TEM}$

275 CONTINU

DLI 375 III $=1,2$

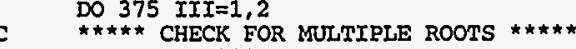

$T E M=E R R N D * 100$.

$\mathrm{NG}=0$

Do $305 \quad I=1,2$

IF (U(I+1)-U(I) -TEM) $300,300,290$

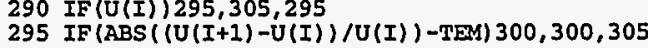

$300 \mathrm{~L}=\mathrm{L}-1$

305 CONTTNUE

IF (LLL-L) $308,400,400$
308 LLL=I

Do 375 II $=1,3$

IF (L) $315,310,322$

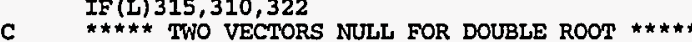

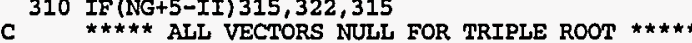

315 DO $320 \quad J=1,3$

VECT $(J, I I)$
GO TO 375

322 D $325 \mathrm{~J}=1,3$

$325 \mathrm{~A}(\mathrm{~J}, \mathrm{~J})=\mathrm{W}(\mathrm{J}, \mathrm{J})-\mathrm{T}$

SMAX $=0.0$

IF (I-2) $335,335,340$

$335 \mathrm{I} 1=\mathrm{I}+1$

$B(I, 1)=A(I, 2) *_{A}(I 1,3)-A(I, 3) \star_{A}(I 1,2)$

$B(I, 2)=A(I, 3) \star_{A}(I 1,1)-A(I, 1) \star_{A}(I I, 3)$
$B(I, 3)=A(I, 1) \star_{A}(I 1,2)-A(I, 2) \star_{A}(I I, 1)$

$T E M=B(I, I) \star \star 2+B(I, 2) \star \star 2+B(I, 3) \star \star 2$

IF (TEM-SMAX) $355,355,350$

SMAX $=$ TEM

IMAX $=I$

355 CONTINUE

1F (SMAX) 353,353,360

$G O$ TO 375

360 SMAX $=$ SORT (SMAX)

DO $365 \mathrm{~J}=1,3$

$365 \mathrm{~V}(\mathrm{~J})=\mathrm{B}(\mathrm{IMAX}, \mathrm{J})$ /SMAX

(

CALL $\operatorname{AXEQB}(A, V, V, 1), \operatorname{ABS}(V(2)), \operatorname{ABS}(V(3))$

DO $370 \mathrm{~J}=1,3$

$370 \mathrm{~V}(\mathrm{~J})=\mathrm{V}(\mathrm{J}) / \mathrm{TEM}$

C CALL UNITY (V, VECT $(1$, II), 1$)$

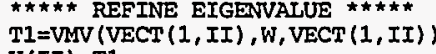

(II)

400 RETURN

END

REAL^8 TD

REAL*8 AIN, ATOMID

CHARACTER*4 TITLE, TITLE2

COMMON NG, $A(9), \operatorname{AA}(3,3), \operatorname{AAREV}(3,3), \operatorname{AANRK}(3,3), \operatorname{AID}(3,3)$

2 , CONT $(5), \mathrm{D}(3,130), \mathrm{DA}(3,3), \mathrm{DP}(2,130)$, DISP, EDGE, FORE, FS $(3,3,96)$

3 , IN, ITILT, KD $(5,20)$, LATM, NATOM, NCD, NJ, NJ2, NOUT, NSR, NSYM

, ORGN (3), PAC $(3,5), \operatorname{PAT}(3,3), Q(3,3), \operatorname{REFV}(3,3), \operatorname{RES}(4), \operatorname{RMS}(5), \operatorname{SCAT} 1$

, SCAL2, SCL, SYMB $(3,3)$, TAPER, THETA, TITLE (18), TITLE2 (18), TS $(3,96)$

6 , VIEW, VT $(3,4), \operatorname{V1}(4), \operatorname{V2}(3), \operatorname{V3}(3), \mathrm{V} 4(3), \mathrm{V} 5(3), \mathrm{V} 6(3), \operatorname{WRKV}(3,3)$

,XING(3),Xo(3),XT(3) character $\star 63$

1 'No sentinel found after reading 96 symmetry cards'.

'No sentinel found after reading parameter cards for 100 atoms',

3 'Aniso temp factor coefs form non-positive definite matrix', 
'Symmetry operation no. is higher than no. of input operations', 5 'Atom number is higher than the number of input atoms'.'

6 'Null temp factor matrix or failure in bisection routine',

7 'Eigenvector routine failure due to null vector',

'Error initializing screen driver'

'Anidentified instruct

'No vector search codes'.

'Insufficient number of atoms in ATOMS list.

'Imaginary bond intersection (i.e., bond larger than atom)'

e 'Hidden (end-on) bond',

$\mathrm{g}$ 'ATOMS array is full'.

h 'Maximum number of overlapping atoms (20) exceeded'.

IF (NOUT GE 0$)$ then

WRITE (NOUT, 115) NG, TD,

115 FORMAT (1H, 10X, 1OHFAULT NG $=, I 3, F 10,0, I 6)$

write (nout, 116) msg(ng)

end if

NG $=0$

RETD

\section{SUBROUTINE EXITNG(ING)}

REAL *8 AIN, ATOMID

CTARACTER TITLE, TITLE

COMMON NG, A $(9), \operatorname{AA}(3,3), \operatorname{AAREV}(3,3), \operatorname{AANRK}(3,3), \operatorname{AID}(3,3)$

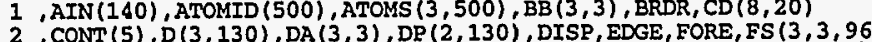

IN, ITILT, KD $(5,20)$, LATM, NATOM, NCD, NJ, NJ2, NOUT, NSR, NSYM

, ORGN (3), PAC $(3,5), \operatorname{PAT}(3,3), Q(3,3), \operatorname{REFV}(3,3), \operatorname{RES}(4), \operatorname{RMS}(5)$, SCAL

5 , SCAL2, SCL, SYMB $(3,3), \operatorname{TAPER}, \operatorname{THETA}, \operatorname{TITLE}(18), \operatorname{TITLE} 2(18), \operatorname{TS}(3,96)$

6 , VIEW, VT $(3,4), \operatorname{V1}(4), \operatorname{V2}(3), \operatorname{V3}(3), \operatorname{VA}(3), \operatorname{VS}(3), \operatorname{V6}(3), \operatorname{VRKV}(3,3)$

,XING (3), XO(3),XT(3)

character 12 xoutin $(18)$

data routin

1 'PRELIM', 'PRELIM' 'PRELIM', 'ATOM, PAXES', 'ATOM, PAXES',

'EIGEN', 'BREEN', 'INITSC ', 'MAIN, SPARE', 'BOMT,

'F500', 'F600, SEARCH', 'BOND', 'BOND', 'F900', 'STORE',

4 'IAP700, LAPAB', 'LAP800, LAPAB'

data msg !

'No sentinel found after reading 96 symmetry cards'

'No sentinel found after reading parameter cards for 100 atoms'

4 'Symmetry operation no. is higher than no. of input operations',

5 'Atom number is higher than the number of input atoms'

6 'Null temp factor matrix or failure in bisection routine'

Eigenvector routine failure due to null vector

. Error inition instion nurber.

a 'Atom out of bounds'.

$b$ 'No vector search codes',

ther than atom).

e 'Hidden (end-on) bond

'Null vector as base line',

h 'Maximum number of overlapping atoms $(20)$ exceeded', if (ng.gt. o) then

f (nout.gt.0) then

write (nouc, 101) ing

write (nout, 102) routin(ing)

write $(*, 101)$ ing

write $(*, 102)$ routin(ing)

end if

,103) msg (ing)

format ('Tau) CLOSE (NOUR, STATUS= ' KEEP'

103

('Subroutine (s) Involved: ',a)

format ('Fault: ', a)

STOP

\section{SUBROUTINE F200}

CHARACTER 4 TITLE, TITLE

COMMON NG, A $(9), \operatorname{AA}(3,3), \operatorname{AAREV}(3,3), \operatorname{AANRK}(3,3), \operatorname{AID}(3,3)$

, CONT (5), D $(3,130), \mathrm{DA}(3,3), \mathrm{DP}(2,130)$, DISP, EDEE, FORE, FS $(3,3,96)$

3 , IN, ITILT, KD $(5,20)$, LATM, NATOM, NCD, NJ, NJ2 , NOUT, NSR, NSYM

$4, \operatorname{ORGN}(3), \operatorname{PAC}(3,5), \operatorname{PAT}(3,3), Q(3,3), \operatorname{REFV}(3,3), \operatorname{RES}(4), \operatorname{RMS}(5)$, SCALI

5 , SCAL2, SCL, SYMB $(3,3)$, TAPER, THETA, TITLE (18), TITLE2 (18), TS $(3,96)$

, VIEW, VT $(3,4), V 1(4), V 2($

,

ent, nvar

$* \hbar \star$
if
if (ndraw.eq. 0$)$ return

To to $(201,202,201,204,205), n j 2$

*** initialize plotting (201 or 203 inst) $* * *$

201 xtrans $=0$.

if (ndraw .eq. 1) call initsc

if ndraw

if (ndraw .eq. 3) call inithp

(naraw

' TEP.EDT' , status = ' unknown' end if end if

$c * \star *$ change origin of plotting area or terminate (202 inst) $* \star \star$

202 if (ain(1) .eq. 0 , and ain(2) .eq. 0.) then

if (ndraw .eq. 2) call endp

if (ndraw ieg. 3) call endhp

if (ndraw .eq. 9) close (npf)

$x$ trans $=$ ain $(1)$
ytrans=ain $(2)$

if (ndraw .eq. 9) write (npE, 203) xtrans, ytrans

203 end if

c $* * *$ change plot color $(204$ inst $) * * *$
204 icolor=ain(1)

if (ndraw .eq. 1) call colrsc(icolor) 
if (ndraw .eq. 2) call colrps (icolor if (ndraw eq. 3) call colrhp (icolor)

return

$c * * *$ parameter width $(205$ inst)

205 penw=ain (1)

if (ndraw .eq. 1) call penwsc (penw)

if (ndraw .eq. 2) call penwps (penw)

if (naraw .eq. 3) call penwhp (penw)

return

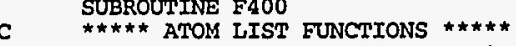

$\mathrm{REAL} \star 8 \mathrm{D} 100, \mathrm{D} 1000, \mathrm{D} 100 \mathrm{~K}, \mathrm{TD}, \mathrm{TD} 1, \mathrm{TD} 2$

COMMON NG A (9) AA $(3,3), \operatorname{AAREV}(3,3)$, AAWRK $(3,3), A T D(3,3)$

, AIN (140), ATOMID (500), ATOMS $(3,500), \operatorname{BB}(3,3), \operatorname{BRDR}, \operatorname{CD}(8,20)$

$, \operatorname{CONT}(5), D(3,130), D A(3,3), \operatorname{DP}(2,130), D I S P, E D G E, \operatorname{FORE}, \operatorname{FS}(3,3,96)$

, IN, ITILT, KD $(5,20)$, LATM, NATOM, NCD, NJ, NJ2, NOUT, NSR, NSYM

, ORGN (3), PAC (3,5), PAT (3, 3$), Q(3,3), \operatorname{RENV}(3,3), \operatorname{RES}(4), \operatorname{RMS}(5)$, SCAL 1

5 , SCAL2, SCL, SYMB (3,3), TAPER, THETA, TITLE (18), TITLE2 (18), TS (3,96)

, XING (3), XO (3), XT (3)

INTEGER $2_{2}$ IDEN

COMMON /PARMS/ CHEM $(505), \operatorname{EV}(3,505), P(3,505), \operatorname{PA}(3,3,505)$

1, IDENT $(2,505)$, MAXATM

D100 $=100$.

$\mathrm{D} 100 \mathrm{~K}=100000$.

$$
\text { IF (LATM) } 402,402,400
$$

400 DO 401 I=1, LATM

CALL ATOM(ATOMID (I), ATOMS $(1, I))$

IF (NOD (NJ2,10)-1) $499,404,403$

$$
\text { GO TO } 499
$$

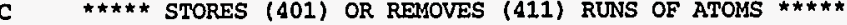

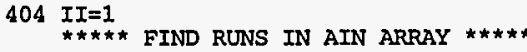

C 405 TD1 $=$ ATN(II) 110,420

$10 \mathrm{IF}$ (TD1) $\mathrm{II}=\mathrm{II}+1$

410 IF $(140-I T) 499,405,405$

$420 \mathrm{JJ}=\mathrm{II}$

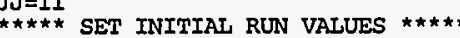

$M 1=T D 1 / D 100 K$

$M 2=D M O D(T D 1, D 100)$

$422 \mathrm{MS}=555$

$M 4=M O D(M 5 / 10,10)$

$M S=M O D(M 5,10)$

$425 \mathrm{JJ}=\mathrm{JJ}+1$

IF $(140-\mathrm{JJ}) 435,430,430$
430 TD2 $=-$ AIN (JJ)

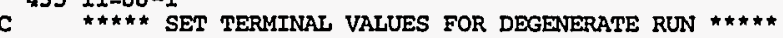

$\mathrm{N} 1=\mathrm{M} 1$

$N 4=M 4$

NO TO 450

$440 I$

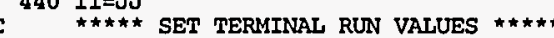

$\mathrm{N} 1=\mathrm{TD} 2 / \mathrm{D} 100 \mathrm{~K}$

$\mathrm{N2}=\mathrm{DMOD}(\mathrm{TD} 2, \mathrm{D} 100)$

NS=DMOD (TD2 $/ D 100, \mathrm{D} 1000$

$445 \mathrm{~N} 5=555$

N5 $=$ MOD $(N 5,10)$

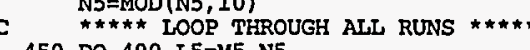

450 DO 490 L5=M5, N5

DO 490 is $=14, \mathrm{ML}$

DO 490 I $=\mathrm{M} 2, \mathrm{Mr}$

DO $490 \mathrm{~L} 1=\mathrm{M1}, \mathrm{N1}$

$T D=D B L E(L 1) * D 100 K+D B L E(L 3 * 10000+L 4 * 1000+L 5 * 100+L 2)$

CALI ATOM(TD, V1 (1)

455 CALL ERPNT (TD, 401)

CO 000

458 IF (MOD (NJ2, 10) -7) 475,460,490

460 IR1=IDENT $(1, \mathrm{~L} 1)$

ID2 =IDENT $(2, L 1)$

45 IF (NCD) $490,490,465$

DO $470 \mathrm{~J}=1, \mathrm{NCD}$

if $(k d(1, j)$.gt. 0 , and. $k d(3, j)$.gt. 0, then

\& (id2.ge.kd $(3, j)$ and. id1.le.kd $(2, j))$.and.

else if (ka(1,j).gt.0.) then 1 the $k d(2, j))$ go to 475 if (idl.ge.kd $(1, j)$ and.

else if $(\mathrm{kd}(3, j), \mathrm{gt}, 0$.$) then \mathrm{k}$. $\mathrm{kg}(4, j))$ go to 475 end if

c $\operatorname{IF}(\operatorname{ID1}-\mathrm{KD}(1, \mathrm{~J})) 470,467,467$

468 IF (ID2-KD (3,J) $470,469,469$

$469 \operatorname{IF}(\mathrm{KD}(4, J)$-ID2) $470,475,475$

470 CONTINUE

GO TO 490

475 CALL STOR(TD)

GO TO 410

499 RETURN

SUBROUTINE F500

DIMENSION RM $(3,3), \mathrm{V}(3,4)$
REA *8 TD, D100, D1000, D100

作 
COMMON NG, $A(9), \operatorname{AA}(3,3), \operatorname{AAREV}(3,3), \operatorname{AANRK}(3,3), \operatorname{AID}(3,3)$

, AIN 140), ATOMID $(500), \operatorname{ATOMS}(3,500), B B(3,3), \operatorname{BRDR}, \mathrm{CD}(8,20)$

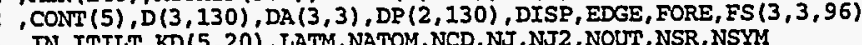

, ORGN (3), PAC $(3,5), \operatorname{PAT}(3,3), Q(3,3), \operatorname{REFV}(3,3), \operatorname{RES}(4), \operatorname{RMS}(5), \operatorname{SCALI}$

,

VTEN, $(3,4)$ V1 (4) V2 (3),V3(3),V4(3), V5 (3), V6 (3), WRKV $(3,3)$

$, X I N G(3), X O(3), X T(3)$

INTEGER 2 IDENT

$(505), \operatorname{EV}(3,505), P(3,505), \mathrm{PA}(3,3,505)$

$\mathrm{NG}=0$

$D 1000=1000$

$100 \mathrm{~K}=100000$.

IF (NJ2-11) 500,

500 IF (NJ2-1)

CAT ATOM(TD,ORGN)

CALI ATS $502,504,502$

CALI ERPIT (TD, 501

CALI EXITNG (NG)

504 DO $506 \mathrm{~K}=1$,

$T D=A I N(K+1), V(1, K)$

CALL ATOM(TD, $V(1, K)$

506 CONTINUE

DO $507 \mathrm{~J}=1,3$

$\begin{array}{rl}\text { V1 }(J) & =V(J, 2)-V(J, 1) \\ 507 & V 2(J)=V(J, 4)-V(J, 3)\end{array}$

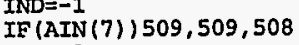

508 IND $=-2$

GO TO 670

C $\star \star \star \star \star$ SHIFT ORIGIN FOR PROJECTION AXIS (IN INCHES) $\star \star \star \star \star ~$

511 DO $513 \mathrm{~J}=1,3$ DO $512 \mathrm{~K}=1$

512 ORGN $(J)=O R G N(J)+R E F V(J, K) * T 1 / S C A L$

$T 2=A \operatorname{IN}(\mathrm{J})$

GO TO 675

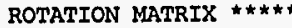

515 DO $514 \mathrm{~J}=1$, 3

$514 \mathrm{~V}(\mathrm{~J}, \mathrm{X})=\mathrm{REFV}(\mathrm{J}, \mathrm{K})$

DO $517 \quad \mathrm{~L}=1,139,2$

I=AIN(L)

IF (I) $532,519,516$

$516 \mathrm{X}=\mathrm{AIN}(\mathrm{L}+1) * 1.7453293 \mathrm{D}-2$

$T 1=\cos (x)$

$T 2=\operatorname{SIN}(X)$

$I 3=\operatorname{MOD}(I+2,3)+1$
$I 1=\operatorname{MOD}(I 3,3)+1$

$I 2=\operatorname{MOD}(I 1,3)+1$

RM(II II) $=\mathrm{T} 1$

$\mathrm{RM}(\mathrm{I1}, \mathrm{I2})=\mathrm{T2}$
$\mathrm{RM}(\mathrm{I} 1, \mathrm{I3})=0.0$
$R M(I 2, I 1)=-T^{2}$

$R M(I 2, I 2)=T 1$

$\mathrm{RM}(\mathrm{I3}, I 2)=0.0$

$R M(I 3, I 3)=1.0$

517 CALI MM(V, RM, V)
519 IF (NJ2-3) 518, 525, 599

519 IF (NJ2-3) 518 DO $522 \mathrm{~J}=1,3$

$522 \operatorname{REFV}(I, J)=V(I, J)$

GO TO 552

525 DO $528 \quad J=1,3$

DO $528 \quad I=1,3$

WRKV $(I, J)=V(I, J)$

GO TO 552

535,552,599

DO $542 \mathrm{~J}=1$,

DO $542 \mathrm{~K}=1,3$

$T 1=R E F V(K, 3)$

$\operatorname{REFV}(\mathrm{K}, 3)=\operatorname{REFV}(\mathrm{K}, 2)$

$\operatorname{REFV}(K, 2)=\operatorname{REFV}(K, 1)$

$542 \operatorname{REFV}(K, 1)=$

IF (NJ2-3) 670,582,599

52 CALL MM (AA, WRKV, AANRK)

GO TO 699

599 IF (NJ2-5) 699,600,607

$600 \operatorname{IF}(\operatorname{LATM}-1) 605,610,610$

606 CALL ERPNT (0.DO,506)

CALI EXITNG (NG)

IF (NJ2-6) 699, 608,710

IF (LATM-3) 605, 610, 610

DO $612 \mathrm{~J}=1$,

DO $612 \quad I=1,3$

DO $620 \mathrm{~K}=1$, LATM

$$
\mathrm{T} 2=1.0
$$

MID $(K), \operatorname{ATOMS}(1, K)$

IF (NCD) $618,618,613$

作 $616 \mathrm{~J}=1, \mathrm{NCD}$

if $(\operatorname{kd}(5, j)$. eq. 1$)$ i1 $=i \operatorname{den} t(1, k)$

if $(\mathrm{kd}(5, j), \mathrm{eg}, 2)$ i1=ident $\langle 2, k)$

$=\operatorname{CD}(1, \mathrm{~J})$

$\operatorname{IF}(\operatorname{II}-\mathrm{KD}(1, J)) 616,614,614$

616 CONTINUE

18 TO 620

DO $619 \mathrm{~J}=13$

19 V2 $(\mathrm{J})=\mathrm{V} 2(\mathrm{~J})+\mathrm{ATOMS}(\mathrm{J}, \mathrm{K}) * \mathrm{~T} 2$

620 CONTINUE

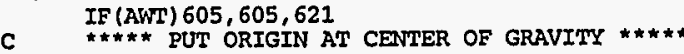

621 DO $622 \mathrm{~J}=1,3$ 
IF $(\mathrm{NJ2} 2-6) 699,624,710$

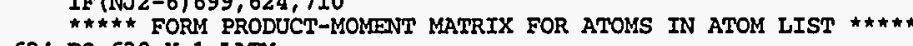

$$
\mathrm{T} 2=1.0
$$

IF(NCD) $628,628,625$

II=ATOMID (K) $/ \mathrm{DIOOK}$

if $(k a, 5, j)$ eq. 1$)$ il=ident $(I, k)$

if $(\mathrm{kd}(5, \mathrm{j})$.eq. 2$)$ i1=ident $(2, \mathrm{k})$

(I)

626 IF (KD $(2, J)-I 1) 627,626,62$

627 CONTINUE

GO TO 630

DO $629 \mathrm{~J}=1,3$

$\mathrm{T} 1=(\operatorname{ATOMS}(\mathrm{J}, \mathrm{K})-\mathrm{ORGN}(\mathrm{J})) * \mathrm{~T} 2$

DO

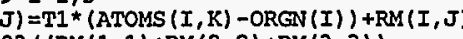

630 CONTINUE

$$
\text { DO } 632 \quad J=1,3
$$

$632 \underset{* \star \star \pi *}{\operatorname{RM}(I, J)=R M(I, J) * I I}$ TRR

CALL MM (RM, AA, DA)

IF (RMS (2)) 605,605,635)

635 IF (NG) $640,633,606$

633 $* * * *$ MAKE SURE VECTORS ARE ORTHOGONAL $\rightarrow>$ NEW REFERENCE VECTORS

33 CALL AXES (PAT $(1,3), \operatorname{PAT}(1,1), \operatorname{REFV},-1)$

Go To 665

G

640 IF (NG+6)

CAIL UNITY (PAT $(1, N), V 1,-1)$

DO $650 \mathrm{~K}=1,3$

IF (ABS (VMV (V1, AA, REEV (1,K))) - .58) 655, 650, 650

650 CONTINUE

$\operatorname{XES}(V 1, \operatorname{REFV}(1, K), D A,-1)$

$\mathrm{D} 660 \mathrm{~K}=1,3$

DO $660 \mathrm{~J}=1,3$

$660 \operatorname{REEV}(\mathrm{J}, \mathrm{L})=\mathrm{DA}(\mathrm{J}, \mathrm{K})$

670 CALI MM (AA, REFV, AAREV)

675 DO $680 \quad \mathrm{~J}=1,3$

$$
\text { WRKV }(I, J)=\operatorname{REFV}(I, J)
$$

$680 \operatorname{AAWRK}(I, J)=\operatorname{AAREV}(I, J)$

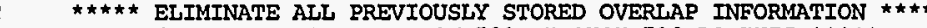
699 CALL LAPSOO (0)

GO TO 710

700 CALL LAPSO0 (1)

710 RETURN

c SUBROUTINE F600

DIMENSION MAX (3), SCAI (4),X (3), XMAXX (3), XMIN (3)
CHARACTER*4 TITLE, TITLE2

COMMON NG, A $(9), \operatorname{AA}(3,3), \operatorname{AAREV}(3,3), \operatorname{AAWRK}(3,3), \operatorname{AID}(3,3)$

(

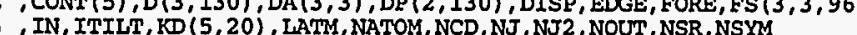

$, \operatorname{ORGN}(3), \operatorname{PAC}(3,5), \operatorname{PAT}(3,3), Q(3,3), \operatorname{REFV}(3,3), \operatorname{RES}(4), \operatorname{RMS}(5), \operatorname{SCAL} 1$

5 , SCAL2, SCL, SYMB $(3,3)$, TAPER, THETA, TITLE (18), TITLE2 $(18), \operatorname{TS}(3,96)$

6 , VIEN, VT (3, 4), V1 (4),V2 (3),V3 (3),V4 (3),V5 (3),V6 (3), WRKV $(3,3)$ , Xeing (3), xo(3), XT(3)

dimension crtval $(99)$

$10.3389,0.4299,0.4951,0.5479,0.5932,0.6334,0.6699,0.7035$ $0.7349,0.7644,0.7924,0.8192,0.8447,0.8594,0.8932,0.9162$ $30.9386,0.9605,0.9818,1.0026,1.0230,1.0430,1.0627,1.0821$ $1.1012,1.1200,1.1386,1.1570,1.1751,1.1932,1.2110,1.2288$ $1.2464,1.2638,1.2812,1.2985,1.3158,1.3330,1.3501,1.3672$ 1.3842, 1.4013, 1.4183, 1.4354, 1.4524, 1.4695, 1.4866, 1.5037 $1.6616,1.6797,1.6980,1.7164,1.7351,1.7540,1.7730,1.7924$ $1.8119,1.8318,1.8519,1.8724,1.8932,1.9144,1.9360,1.9580$ $1.9804,2.0034,2.0269,2.0510,2.0757,2.1012,2.1274,2.1544$ b $2.1824,2.2114,2.2416,2.2730,2.3059,2.3404,2.3767,2.4153$,

FOR RECUMENTING FUNCTIONS *t**

DEL $=$ FLOAT (MOD (NJ2/10,2))

NI2 $=$ MOD (NJ2,10) $T 1=\operatorname{ArN}(1)$

IF (T1) $602,604,602$

$602 \quad \mathrm{XO}(1)=\mathrm{T} 1+\mathrm{XO}(1) \star \mathrm{DEL}$
$604 \mathrm{~T} 2=\mathrm{AIN}(2)$ IF (T2) 606, 608, 606

$606 \mathrm{XO}(2)=\mathrm{T} 2+\mathrm{XO}(2) \star \mathrm{DEL}$

$608 \mathrm{~T} 3=\operatorname{AIN}(3)$

609 IF (T3) $612,612,609$

609 IF (DEL) $611,611,610$

GO TO 612

611 SCAL1 $=T 3$

IF (T4) $615,616,614$

C 614 SCAL2=T4 ELLIPSOID SCALE EACTOR $\star * * * * *$

615 go to 616

$$
\text { if }(t 4 \text {. }
$$

AND/OR SCALE $* * * * *$

616 IF (NJ2-2) 790,622, 62

XO(1) =XING (1) *.

$x=250$

625 SCAL $1=1$.

(1) $635,635,640$

635 NG=12

CALL ERPNT $(0$. D0, 602)

640 DO $650 \mathrm{~J}=1,3$ 
$\operatorname{XMAX}(J)=-1 . E 5$

C $\star \star \star \star \star \star$ FIT BOX AROUND SET OF ATOMS $\star \star \star \star \star *$

DO $670 \quad I=1$, LATTM

CALL XYZ (ATOMID (I), ATOMS $(1, I), 3)$

652 CALI ERPNT (ATOMID (I), 600)

653 DO $668 \mathrm{~J}=1$,

DO $668 \mathrm{~J}=1,3$

D.

$655 \operatorname{XMAX}(J)=T 1$

$\operatorname{MAx}(J)=I$

$660 \operatorname{IF}$ (T1-XMIN (J) ) 665, 668,668

(

668 CONTIN

670 CONTINUE

$\mathrm{KMM}=\operatorname{MAX}(3)$

DO $780 \quad M=1,5$

$\operatorname{IF}(M-2) 740,675,678$

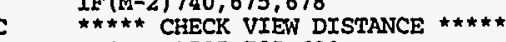

675 IF (VIEW) 785, 785, 680

678 IF (NJ2-3) $680,785,680$

IF (VIEN* .5-T1) $685,690,690$

C $\star * \star \star \star$ INCREASE VIEW DISTANCE $* \star \star \star * *$

685 VIEW $=2$. *T1

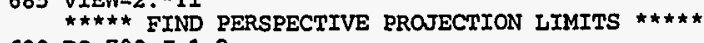

XMAX $(J)=-1,2$

$700 \operatorname{XMIN}(J)=1 . E 5$

DO $725 I=1$, LATM

DO $705 \mathrm{~J}=1,3$

$705 \mathrm{X}(\mathrm{J})=\mathrm{ATOMS}(\mathrm{J}, \mathrm{I}) *$ SMUL

IIEW/ (VIEW-X(3)

$\mathrm{T} 1=\mathrm{X}(\mathrm{J}) \times \mathrm{T} 2$

IF $(\operatorname{MMAX}(J)-T 1) 710,715,715$

$710 \operatorname{XMAX}(J)=T 1$

$715 \mathrm{IF}$ (T1-XMIN (J) ) 720,725,725

$720 \operatorname{XMIN}(J)=T 1$

C $4 * \star \star \star$ REFINE PARAMETERS $\star * \star \star \star *$

740 IF (NJ2 -3) 745, 742, 755

42 SMULL $2=1$.

$$
\text { GO TO } 765
$$

C

75 DO $750 \mathrm{~J}=1,2$

$\mathrm{T} 2=\mathrm{XO}(\mathrm{J})$

(T)

R-T2)/XIAAX (J) , SCAL (2), SCAL (3), SCAL (4)) GO TO 78

C $\star \star \star \star \star$ AUTOMATIC SCALE AND POSITION $* \star \star \star \star *$

755 DO $760 \mathrm{~J}=1,2$

$60 \operatorname{SCAL}(J)=\left(X \mathrm{XING}(J)-\operatorname{BRDR} \mathbf{R}^{\star 2}.\right) /(\operatorname{XMAX}(J)-\operatorname{XMIN}(J))$ SMUL2 $=$ AMIN1 ( SCAL (1), SCAL (2))

765 D $770 \mathrm{~J}=1,2$
$770 \times O(J)=.5 *(X T N G(J)-\operatorname{SMIR} 2 *(\operatorname{XMAX}(\mathrm{J})+\operatorname{XMTN}(J)))$

SMULT=SMULT*SMUL2

VIEN=VIEN*SMUL2 2

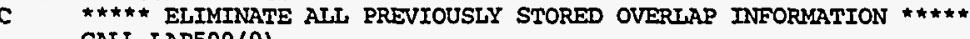
CALL LAP500(0)

RETURN

SUBROUTTNE F700

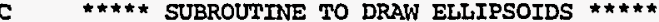

DIMENSION EYE (3), VIEWV (3), X(3), Z(3)

REAL*8 TD, TD2, D100, D1000, D100K

REAL*8 AIN, ATOMID

CHARACTER $* 8$ CHEM

CHARACTER * 8 CHEM $(3,3), \operatorname{AAREV}(3,3), \operatorname{AANRK}(3,3), \operatorname{AID}(3,3)$

1, AIN (140), ATOMID (500), ATOMS $(3,500), \mathrm{BB}(3,3), \mathrm{BRDR}, \mathrm{CD}(8,20)$

$, \operatorname{CONT}(5), \mathrm{D}(3,130), \mathrm{DA}(3,3), \mathrm{DP}(2,130), \mathrm{DISP}, \operatorname{EDGE}, \operatorname{FORE}, \operatorname{FS}(3,3,96)$

3 , IN, ITILT, KD $(5,20)$, LATM, NATOM, NCD, NJ, NJ2 2, NOUT, NSR, NSYM

, ORGN (3), PAC (3, 5), PAT (3, 3), Q (3, 3), REFV (3, 3), RES (4), RNS (5), SCAL1

'SCAL2, SCL, SYMB (3,3), TAPER, THETA, TITLE (18), TITLE2 (18), TS (3,96) Xo(3), XT(3)

CONMON /PARMS/ CHEM(505), EV $(3,505), P(3,505), \operatorname{PA}(3,3,505)$

, IDENT $(2,505)$, MAXATM

common /ns/ npt, ndraw, norient, nvar

c common 7 trfac/ xtrans, ytrans

$D 100=100$.

D100K $=100000$.

ITILT $=0$

NG $=0$

NFIRST $=1$

IF (NPLANE-1) $720,715,720$

715 NFIRST $=4$

720 NSOLID $=$ AIN (2)

NDOT $=64 / 2 \star \star$ (IABS (NSOLID) )

LINES $=A I N(3)$

CHSYM $=$ AIN (5)

$T 6=\operatorname{AIN}(6)$

$\mathrm{DH}=\mathrm{T} 6-\mathrm{CH}$

(7)

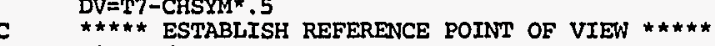

$\mathrm{T} 1=1$, E6

(TEN) $740,740,735$

$735 \mathrm{~T} 1=\mathrm{VIEN} / \mathrm{SCAL}$

$741 \operatorname{EYE}(J)=\operatorname{REFV}(J, 3)$ *T1+ORGN (J)

C LNS $\star-1$ LOOP THROUGH ATOM LIST $\star \star \star \star * \star *$

DO 1105 ITOM $=1$, LATM

TD $=$ ATOMID (ITOM)

IF (AIN (10) ) 744, 744, 7412 
$7412 \operatorname{TF}$ (AIN(12)-1.0DO) 742,7414, 7416

7414 TD2 $=$ IDENT $(1, K)$

7416 TD2 $=$ IDENT $(2, K)$

742 TD2 $2=\mathrm{DINT}$ (TD/D100K)

7422 IF (TD2-AIN (10)) 1105,743,

743 IF(AIN (11)-TD2) 1105, 744,74

IF

746 CALI PLTYY (X

$L=D M O D(T D / D 100, D 1000)$

$L 1=D M O D(T D, D 100)$

if (ndraw.eq.1) WRITE (npf, 750) CHEM(K),K,L,L1

\& $\operatorname{IF}(\mathrm{NJ} 2-10) 747,754,754$

$z(1)+x \operatorname{trans}, z(2)+y$ trans

47 LNS=MOD (LNS +1, 18)

748 IF (NOUT.GE.0)

ShRITE (NOUT, 751) (TITLE (I), I=1, 18)

IF (NOUT.GE.0)

\&WRITE (NOUT, 752)

GWRITE (NOUT, 750) CHEM(K), K, L, L1, Z (1), Z (2)

(3, $1 \mathrm{H}, \mathrm{I3}, \mathrm{I2}, 4 \mathrm{H}) \quad 2 \mathrm{~F} 8.2,3 \mathrm{X}, 3 \mathrm{~F} 8.3,11 \mathrm{X}, 3 \mathrm{~F} 8.4$ )

FORMAT (1H 10X, A6, 3H

$2 \mathrm{~F} 8,2,3 \mathrm{X}, 3 \mathrm{~F} 8.3,11 \mathrm{X}, 3 \mathrm{~F} 8.4$ 1RTESIAN $X, Y, Z$ (IN, ) $15 X, 20 \mathrm{HCRYSTAL}$ SYSTEM $X, Y, Z / 1 \mathrm{H}$ 19X, $45 \mathrm{H}$ (DIRECTIO 3 D ON WORKING SYSTEM/1H)

$55,760,760$

758 CALL ERPNT (TD, 700)

GO TO 1105

ICQ $=0$
CALI LAP700 (ITOM, ICQ)

IF (ICQ) 762,764,764

ATOM $* * * * *$

GO TO 758

764 IF (CHSYM) 775, 775, 765

C

IF (VIEW) 767, 767,766

766 T4=VIEW/ (VIEW-X (3)

(

$\mathrm{V} 1(1)=\mathrm{X}(1)+\mathrm{DH} * \mathrm{SYMB}(1,1)+\mathrm{DV} * \operatorname{SYMB}(1,2)$

$V 1(2)=X(2)+D H * \operatorname{SYMB}(2,1)+D V^{*} \operatorname{SYMB}(2,2)$

V1 $(3)=\mathrm{X}(3)$

CALL $\operatorname{PLTXY}(v 1, \sqrt{ } 3)$

768 V2 $(3)=0$.
$\mathrm{V} 2(1)=\mathrm{V} 3(1)+$ FLOAT $(I-2) \star T 4$
$\mathrm{V} 2(2)=\mathrm{V} 3(2)+\mathrm{FLOAT}(\mathrm{J}-2) \star \mathrm{T} A$
CALL SIMBOL (V2 (1), V2 (2), T3, CHEM (K) , THETA, 6)
IF (T4) 775, 775, 770

770 CONTINUE

$1105,1105,780$

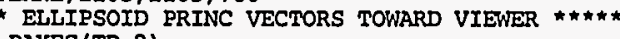

CALL PAXES (TD, 2)

783 CALL DIFV (EYE, XT, VIEWU)

CALL UNITY (VIEW, VIEWV, -1 )

CALL VM(VIEWV, AA, V2)

TF(VV(V2, PAT (1,I))) 785, 795,795

785 DO $790 \mathrm{~J}=1,3$

$\operatorname{PAC}(J, I)=-\operatorname{PAC}(J, I)$

$790 \operatorname{PAT}(J, I)=-\operatorname{PAT}(J, I)$

795 CONTINUE

$$
D 0800 \mathrm{~J}=1,3
$$

000 PAC $(U, 4)=\operatorname{saC}(U, 1)$

IFE(NT2-10)802,803,803

801 FORMAT (1H , 13X, 3(3X, 3F8.4, F8.5)/1H $)$

SWRITE (NOUT, 801) ( $P A C(J, K), J=1,3)$, RMS (K) $, K=1,3$ )

C $803 * \pi * *$ V4

continue
CALI VM(VIEWV, AAWRK, V6)

CALL UNITY (V6, V6, 1 ,

C CALL UNITY (V4, V4, 1)

t3a=sqrt (rms (3)* $\operatorname{rms}(2))$

t3b=sqre (rms $(2) * 2 \mathrm{~ms}$ (1)

t3c=sqxt (2ms $(3) * \mathrm{rms}(1))$

(t3c) $/ 3.0) * S C L$

NO $805 \mathrm{~J}=1,3$

IF (T3-RES (J) ) 804, 810, 810

804 NBIS $=$ NBIS -1

805 NRESOL $=$ NRESOL $* 2$

810 NRES1=NRESOL+1

DO 1100 II =NFIRST, NPLANE

II $1=$ MOD $(I I, 3)+1$

II $2=\operatorname{MOD}(\operatorname{II}+1,3)+1$

+1

$\operatorname{IF}(.99938-\mathrm{ABS}(\mathrm{VV}(\mathrm{V} 4, \mathrm{PAC}(1, \mathrm{II} 2)))) 820,820,830$

820 (T)

$$
\text { DO } 825 \mathrm{~J}=1,3
$$

$D A(J, 1)=P A C(J, I T O) *$

$825 \mathrm{DA}(\mathrm{J}, 2)=\operatorname{PAC}(\mathrm{J}$, III $) \star T 2$

GO TO 850

830 CALL NORM(PAC $(1$, IIO $), \operatorname{PAC}(1$, III), V1, 1)

CALL NORM(V1, V4, V2,

CALL NNTHY (V2,V2,1)

835 CALL NORM(V3, V1, V5, 1)

GO TO 843

840 CALL NORM(V3,V4,V5,1)
843 CALL UNITY (V5, V5, 1$)$ 
$\mathrm{T} 1=\mathrm{SCL} / \mathrm{SQRT}(\mathrm{VMV}(\mathrm{V} 2, \mathrm{Q}, \mathrm{V} 2))$

DO $845 \mathrm{~J}=1,3$

$\mathrm{DA}(\mathrm{J}, 1)=\mathrm{V} 2(\mathrm{~J}) * \mathrm{~T}$

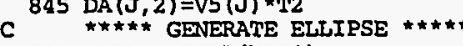

850 CALL RADIAL (NBIS)

851 IF(NSOLID) $859,859,852$

C $\star \star \star \star \star$ PIOT DOTTED BOUNDARY ELTTPSE *****

52 IF (NDOT-NRESOL) 853, 855, 855

853 CALL RADIAL (NSOLID-1)

55 CALI PROJ (D,DP, X,XO,VIEW, 1, 129, NDOT)

CALI DRAW(DP (1, J), DISP, DISP, 3)

DO $856 \quad I=1,3,2$

DO $856 \mathrm{~K}=1,3,2$

T2 $=$ FLOAT $(\mathrm{K}-2) * \mathrm{DISP}$

CALL DRAW (DP $(1, \mathrm{~J})$

856 CONTINUE

\section{GO TO 1100}

C $\star * \star \star \star *$ PLOT SOLID BOUNDARY ELLIPSE $\star \star \star \star \star * *$

859 CALL PROJ (D, DP, X, XO, VIEW, 1, 129, NRESOL)

CALL DRAW (DP, $0 ., 0 ., 3)$

860 CALL DRAW(DP $(1, J), 0,0,2$, NRESOI

IF (DISP)

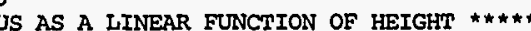

T5 =WV (V1, AREV $(1,3)) * S C A T$

$T 8=A I N(8)$

$T 9=A I N(9)$

NCYCLE $=.5+(T 8+15 * T 9) / D I S P$

$70 \mathrm{~T}^{3}=(2 *$ *ISP) $/(\mathrm{T} 1+\mathrm{T} 2)$

C70 $* \star \star \star \star$ INCREASE ANNULAR THICKNESS $\star \star \star \star \star *$

DO $875 \quad I=1$, NCYCLE

$T 4=T 3^{*} F L O A T$ (I)

875 CALL DRAW $(D P(1, J), D(1, J) * T 4, D(2, J) * T 4,2)$

900 CALL PROJ (D, DP, $X, X O$, VIEW, 1,65 , NRESOL)

C 900 CALL PROJ(D, DP,, X, XO, VIEW, 1,65, NRE

CALL DRAW (DP, $0 . .0 ., 3)$

DO 905 J=NRES1, 65, NRESOL

905 CALL DRAW(DP $(1, J), 0 ., 0,2$ )

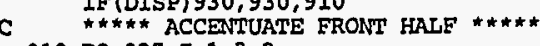

10 DO $925 \quad I=1,3,2$

DO $915 \mathrm{~J}=1,65, \mathrm{NRESOL}$

$\mathrm{K}=66-\mathrm{J}$

915 CALL DRAW (DP (1,K),DISP, T2, 2)

DO $925 \mathrm{~K}=1,65$, NRESOL

925 CALL DRAW (DP (1, K), -DISP, -T2,2)

930 IF (NSOLID) $940,967,935$

IF(NDOT-NRESOL) $938,945,940$
938 CALL RADTAL (NSOLID-1)

GO TO 945

945 CALL PROJ $(D(1,65), D P(1,65), X, X 0, V I E W, 1,65, \mathrm{~L})$

IF (NSOLTD) $960,967,950$

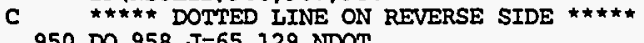

50 DO $958 \mathrm{~J}=65,129$, NDO

CALI DRAW(DP $(1, J), D I S P, D I S P, 3)$

DO $955 I=1,3,2$

DO $955 \mathrm{~K}=1,3,2$

T2 $=$ FLOAT $(K-2) * D I S P$

CALL DRAW(DP $(1, J), T 1, T 2,2)$

955 CONTINUE

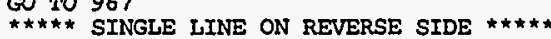

$960 \mathrm{DO} 965 \mathrm{~J}=65,129$, NRESOL

965 CALL DRAW (DP $(1, J), 0, \ldots 0,2)$

$967 \mathrm{T2}=\mathrm{NDASH}{ }^{*}$

DO $975 \mathrm{~J}=1,3$

$\mathrm{DA}(\mathrm{J}, 2)=\mathrm{PAC}(\mathrm{J}, \mathrm{II} 1) * \mathrm{RMS}$ (III) *SCL

$\mathrm{DA}(\mathrm{J}, 3)=0$.

970 V1 (J) $=-175,975,970$

970 V1 $(J)=-T 1$

975 CONIINUE

IF (NDASH) $987,987,980$

DOR REVERSE AXIS $\star \star \star \star * *$

DO $985 \mathrm{~J}=1$, NDASH

$D=4-K \quad K=1,2$

CALL PROJ (V1, DP, X, XO, VIEW $, 1,1,1$ )

CALL DRAW (DP, $0,0, L$ )

$985 \quad I=1,3$

C $* \star \star * \star$ SOLID LINE FOR FORWARD AXIS $\star \star \star * *$

987 IF (LINES) $1100,1100,988$

988 CALL PROJ (DA, DP, $X, X 0, V I E W, 1,3,1$

TI $=$ DISP* .5

T2=ELOAT $\left(2, I^{2}\right.$

CALL DRAW(DR, T1, T2, 3)

CALL DRAW (DP $(1,3), \mathrm{T} 1, \mathrm{~T} 2,2)$

IF (DISP) 1000,1000,989

(1)

990 CALL DRAW (DP, TI, T 2 ;

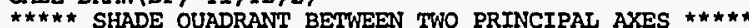

L=LINES-I 1100,1005

1005 T2=LINES

DO $1025 I=1, L$

T1=FLOAT (I) $/ \mathrm{T} 2$

$M=M-1$ 
GO TO 1020

$1015 \mathrm{~N}=\mathrm{T} * 2$

1020 DO $1025 \mathrm{~J}=1,3$

$T A=D A(J, 1) * T 1$

$1025 \mathrm{D}(\mathrm{J}, \mathrm{N})=\mathrm{DA}(\mathrm{J}, 2) * \mathrm{~T} 3+\mathrm{T} 4$

$\mathrm{L}=\mathrm{L} \star 2$

CALI PROJ (D, DP, X, XO, VIEW, $1, L, 1$

CAI $103012,1,2$

CALI DRAW(DP $(1, I-1), 0,0,3^{3}$

1100 CONTINUE

1105 CONTINUE

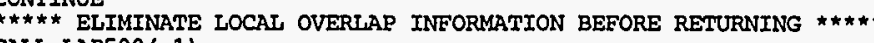
CALL, LAP500(-1)

$$
\text { CAD }
$$

\section{SUBROUTTNE FB00}

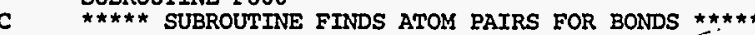

DIMENSION IA (3), W1 (6)

REAL 8 AIN, ATI

CHARACTER 4 ATITIE,

CHARACTER*8 CHEM

COMMON NG, $A(9), \operatorname{AA}(3,3), \operatorname{AAREV}(3,3), \operatorname{AANRK}(3,3), \operatorname{AID}(3,3)$

(3)

D $(3,130), D A(3,3), D P(2,130)$, DISP, EDGE, FORE, FS $(3,3,96)$

, IN, ITILT, KD (5, 20), LATM, NATOM, NCD, NJ, NJ2, NOUT, NSR, NSYM

, 'SCAL2, SCL, SKMB (3,3) TAPER, THETA, TITLE (18), TITLE2 (18), TS $(3,96)$

6 , VIEW, VT $(3,4), \mathrm{VI}(4), \mathrm{V} 2(3), \mathrm{V} 3(3), \mathrm{V} 4(3), \mathrm{V} 5(3), \mathrm{V} 6(3), \operatorname{VRKV}(3,3)$

,XLNG (3), XO(3), XT(3)

,

$1, \operatorname{IDENT}(2,505)$, MAXATM

COMMON/OLAP/CONIC $(7,500), \operatorname{COVER}(6,20), \mathrm{KC}(20), \mathrm{KO}(30), \mathrm{NCONIC}, \mathrm{NCOVER}$

1 NQOVER, NQUAD, OVMRGN, QOVER $(3,4,30)$, QUAD $(9,600), \operatorname{SEGM}(50,2)$

$c * * *$ old

MOD $(N J 2,10)-$

$\operatorname{IAN}=\mathrm{AIN}(2)$

NJ4 $=($ MOD $(N J 2,10)-4)+($ IAN $\star 2)$

if (nj.eq.10) $n j 4=\left(\tan { }^{\star} 2\right)-2$

INS $=-4$ (MOD $\left.\left.(N)^{2}, 10\right)-2\right) 805,848,848$

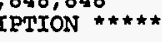

805 II =0

810 NG=11

CALL ERPNT $(0 . \mathrm{DO}, \mathrm{NJ} \star 100+\mathrm{NJ} 2)$

GO TO 980

IF $(140-$ II $) 980,980,820$

820 ID1=ATN(IT)

IF (TD1) 815, 815, 825$$
\text { TD2 }=\text { ATN }(I)
$$

(II) 815,830

$830 \operatorname{IF}($ (NT2 $2-10), 832,838,838$
832 LNS=MOD(LNS+4, 56)

834 IF (LNS) 838,834,8

IF (NOUT.GE.0)

(I) , I=1, 18

(1H1, 10X, 18A4)

IF (NOUT.GE.0)

837 FORMAT (1H010X, 18HSXMBOL ATOM CODE6X, 16HPLOTTER X, $Y$ (IN.) 6X, 21HCA 1RTESIAN $X, Y, Z$ (IN.) 17X, 2OHCRXSIAL SYSTEM $X, X, 2 / 1 H$ )

(330 NAT CHECK IF BOND ATOMS ARE IN ATOMS LIST (FOR OVERLAP CALC) *** $N A 1=0$
$N A 2=0$

IF (LATM-2) 845, 839, 839

$839 \mathrm{~N} 2=2$

DO $844 \mathrm{~K}=1$, LATM

TD3 $=$ ATOMID $(K)$

IF (TD3-TD1) 841, 840,841

840 NA1=K

841 TF (TD3-TD2) $844,842,844$

$842 \mathrm{NA} 2=\mathrm{K}$

$843 \mathrm{~N} 2=\mathrm{N} 2-1$

$\operatorname{IF}(\mathrm{N} 2) 845,845,844$

845 IF (NA2-NA1) $846,847,847$

$\mathrm{NA} 3=\mathrm{NA1}$
$\mathrm{NA} 1=\mathrm{NA2}$

$\mathrm{NA2}=\mathrm{NA3}$

TD3=TD1

$T D 1=T D 2$

847 CALL BOND (TD1, TD2 2, 1, NA1, NA2)

(1)

848 IF (LATM-2) $810,850,850$

DO $855 \dot{I}=1$, LATM

855 CALL XYZ (ATOMID (I), ATOMS $(1, I), 2)$ SCAL1=SCAL

860 IF (NOUT $810,810,860$

SWRTIE (N.GE.0)

861 FORMAT (1H010X, 20HBOND SELECTION CODES//11X, 94H (SEQUENCE (A)) (SEQUEN (BD) (BOND) (DISTANCES) ( BOND ) (RERSP. - LAAEELS) (NORMAL-- (SERUAN 2DIGITS) /11X,93H( MIN MAX / MIN MAX) (TYPE) (MIN MAX) (RAD (HES) (HEIGT

DO $870^{\circ} I=1, N C D$
IF (DMAX-CD $(2, I)) 865,866,866$

865 DMAX $=C D(2, I)$

66 IF (NOUT.GE.0)

70 COTE (NOU

871 FORMAT (1H , 10X, I6, I5, I8, I5, I8, 2F6,2, 5F8.3, F7.0)

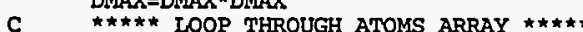

DO $977 \mathrm{M}=1$, IATM

$\mathrm{NA} 1=\mathrm{M}$

TD1 $=$ ATOMID $(M)$

$M I=T D 1 / D 100 K$ 
IF (NJ4) 8722, 8724, 8718

8718 IF (NJ4-2) 8724, 8726, 8720

8724 IA (1) $=$ TDE

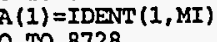

(1) $=\operatorname{IDENT}(2, \mathrm{MI})$

$8728 \operatorname{IA}(3)=\operatorname{IA}(1)$

W1 $(1)=\operatorname{ATOMS}(1, M)$

WI (2) $=\operatorname{ATOMS}(2, M)$

872 DO 975 N=L, LATM

$N A 2=N$

$\operatorname{DIST}=(\operatorname{ATOMS}(1, \mathrm{~N})-\mathrm{WI}(1)) *{ }^{2}$

IF (DMAX-DIST) $975,873,873$

873 DIST $=$ DIST+(ATOMS $(2$, N) $-W 1(2)) \star \star 2$

074 DIST $=D I S T+($ ATOMS $(3, N)-W 1(3)) * \star 2$

IF (DMAX-DIST) 975, 875, 875

DIST $=S Q R T$ (DIST)

TD2 $=A T O M I D(N)$

$\mathrm{NI}=\mathrm{TD} 2 / \mathrm{D} 100 \mathrm{~K}$

C 876 IF (NJ4) 876, 877,878

C 876 IA (2) =TD2

c 877 IA (2) $170 \operatorname{IDENT}(1, \mathrm{NI})$

c 60 TO 879

$\operatorname{IA}(2)=\operatorname{IDENT}(2, \mathrm{NI})$

IF (NJ4.EQ.0.OR. NJ4.EQ.1) IA (2) $=\operatorname{IDENT}(1, \mathrm{NI})$

IF (NJ4.GT.1) IA(2) $=$ IDENT $(2$, NI)

879 DO 950 J $J=1, N C D$

$J B=J$

$880 \operatorname{IF}(C D(2, J)$-DIST) $\quad 950,881,881$

881 DO $885 \mathrm{~K}=1$,

$\operatorname{IF}(\operatorname{IA}(K)-K D(1, J)) \quad 885,882,882$

883 IF (II (KI)

$884 \operatorname{IF}(\mathrm{KD}(4, \mathrm{~J})-\operatorname{IA}(\mathrm{K}+1)) 885,890,890$

5 CONTINUE

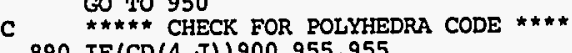

$890 \operatorname{IF}(\operatorname{CD}(4, J)) 900,955,955$

W1 $(4)=$ ATOMS $(1, N)$

W1 $(6)=\operatorname{ATOMS}(3, N)$

$\mathrm{KM}=\operatorname{ABS}(\mathrm{CD}(4, \mathrm{~J})$

$\mathrm{KM} 2=\mathrm{ABS}(\mathrm{CD}(5, \mathrm{~J}))$

$\operatorname{DSQ1}=(C D(6, J))^{* \star 2}$

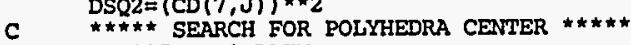

DO 935 IM=1, LATM

if (ian.eq. 1) $\mathrm{k3}=$ ident $(1, \mathrm{im})$
if (ian.eq. 2) $\mathrm{k} 3=$ ident $(2, \mathrm{im})$

IF (K3-KM1) 935, 905, 905

905 IF (KM2-K3) 935, 910,910

DISTANCE RANGE $* * * * *$

$=1,4,3$

DSO=(ATOYS (1, IM) -W1 (J1)) **2

IF (DSQ2-DSQ) 935, 915,915

$915 D S Q=D S Q+(\operatorname{ATOMS}(2, I M)-W 1(J 1+1)) * \star 2$

20 DSQ $=D S 2+$ (ATOMS $(3$, IM) $-W 1(J 1+2)) * \star 2$

IF (DSQ2-DSQ) 935,925,925

GO TO 955

935 CONTINUE

C $\star$,

950 CONTINUE

$$
\text { GO TO } 975
$$

PREPARE TO DRAW BOND $* \star \star \star * *$

LNS $=$ MOD (LNS $+4,56$ )

965 IF (NOUT.GE. 0)

SWRITE (NOUT, 835) (TITLE (I) , I=1, 18)

IF (NOUT.GE. 0)

970 CALL BOND (TD1, TD2, JB, NA1, NA2)

975 CONTINUE

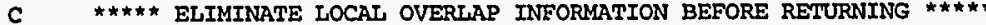

$980 \mathrm{IF}(\mathrm{NJ2} 2-21) 985,990,990$

985 CALI LAPSOO ( -1$)$

990 if (nj2.eq. 22) then

IF (NQUAD)

$\mathrm{C}$

991 IF (NOUT.GE. 0)

992 FORMAT (1H0, 10X, 27HBOND OVERLAP ARRAY CONTAINS, I4, 23H BONDS (MAXIMU

end if

93 RET

SUBROUTINE F900

DIMENSION $X(3), X W(3,5), Y(3), z(3)$

REAL, $* 8$ AIN, ATOMID
CHARACTER $* 4$ TITLE, TITLE2

COMMON NG, A $(9), \operatorname{AA}(3,3), \operatorname{AAREV}(3,3), \operatorname{AAWRK}(3,3), \operatorname{AID}(3,3)$

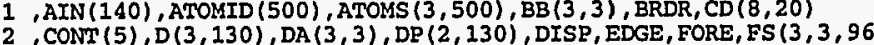

, IN, ITILT, KD $(5,20)$, LATM, NATOM, NCD, NJ, NJ2, NOUT, NSR, NSYM

, ORGN (3), PAC (3,5),

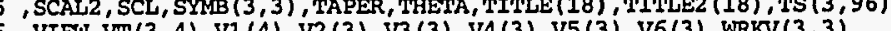

7 XING(3), XÓ(3), XT(3)

COMMON PARMS/ $\operatorname{CHEM}(505), \operatorname{EV}(3,505), \mathrm{P}(3,505), \mathrm{PA}(3,3,505)$

$1, \operatorname{IDENT}(2,505)$, MAXATM

character 72 trmpti, tmpti2 
$\mathrm{D} 100 \mathrm{~K}=100000$.

ITILT $=0$

NJ3=MOD (NJ2,10)

SINTH $=$ SYMB $(2,1)$

ILAST $=1$

$T 2=A I N(2)$

905 ILAST $=2$

910 DO 925 II=1, ILAST

COORDTHATES thtta

CALI XXZ (AIN (II), XW(1,II) ,2)

CALL XYZ (AIN (II), XW(1,II+3), 3 )

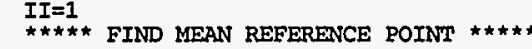

DO $930 \mathrm{~J}=1,3$

$$
T 1=X W(J, 1)
$$

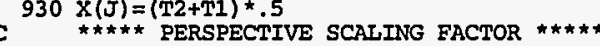
$S C A L=1$.

935 (

$\mathrm{HGT}=\mathrm{SCAL} \star T$

IF (NJ2-3) $960,950,945$

$945 \operatorname{IF}(\mathrm{NJ} 2-6) 950,950,960$

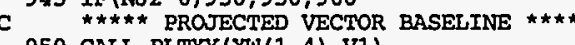

950 CALL PLTXY (XW $(1,4), V 1)$

$\mathrm{CAEL} 1=\mathrm{V} 2(1)-\mathrm{V} 1(1)$
$\mathrm{T} 2=\mathrm{V} 2(2)-\mathrm{V} 1(2)$

$T 3=S R R T(T 1 * T 1+T 2 * T 2)$

IF(T3) $912,912,955$

COSTH $=T 1 / T 3$
STNTH $=$ T2 T T3

SINTH $=T 2 / \mathrm{T} 3$

TH=ARCCOS (COSTH)

$958 \mathrm{TH}=-\mathrm{TH}$

960 IF (NJ2-13

$965 \stackrel{\pi=A \operatorname{TN}(6)}{\pi}$

$T 7=\operatorname{AIN}(7)$

$Y(1)=\mathrm{SCAL}^{*}\left(\mathrm{X}(1)+\mathrm{T} 66^{*} \mathrm{COSTH}-\mathrm{T} 77^{\star} \mathrm{SINTH}\right)+\mathrm{XO}(1)$

$+\mathrm{T} 7 \star \cos T H)+\mathrm{XO}(2)$

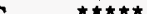

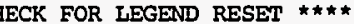

$\mathrm{D} O \mathrm{~T}$
$980 \mathrm{~J}=1,2$

IF (T1) $975,980,970$

$970 \quad Y(J)=T 1$

5 Y T $=$ XING (J) + T 1

980 CONTINU

SER PAPAMETERS FOR TNDTVTDUAL EUNCTTONS *****

985 GO TO $(990,995,995,1000,1000,1000,915,1105,1105,915,915,915,1005,10$ $105,1005,1005,915)$, NJ2

$990 \mathrm{~T} 6=17$.
$\mathrm{L}=\mathrm{AIN}(1) / \mathrm{D} 100 \mathrm{~K}$

IIAST $=1$
DXW $=0$.

DXW=0.

DO 1030

$995 \mathrm{~T} 6=215$.

IIAAST $=18$

$\mathrm{TI}=\mathrm{HGT} \approx 24.17$.

$\mathrm{DXW}=\operatorname{COSTH} * \mathrm{~T} 1$

$D Y W=S I N T H * T 1$

1000 T6 $=10+3 *(\mathrm{NJ} 3-4)$

DIST $=\operatorname{SORT}(V V(X W(1,3), X W(1,3))) / \operatorname{SCAL} 1$

GO TO 1030

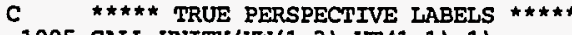

1005 CALL UNITY (XW $(1,3), \operatorname{VT}(1,1), 1)$

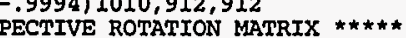

CATL NORM (ATD (1 3) VT(1,1) VT $(1,2)$, 1)

CALL UNTTY (VTT (1, 1 ) VT(1,2), 11

CALL $\operatorname{NORM}(\operatorname{VT}(1,1), \operatorname{VT}(1,2), \mathrm{VT}(1,3), 1)$

$1015 \operatorname{VT}(J, A)=X(J)$

ITILT $=1$

HGT $=A I N(5)$

$\mathrm{T}(3)=\mathrm{X}(3)$
$\mathrm{TT} 7=\mathrm{AIN}(7)$

$Y(2)=X(2)+T T 7-H G T * .5$

$T T 6=A \operatorname{IN}(6)$

IF (NU2-13) $1030,1025,1020$

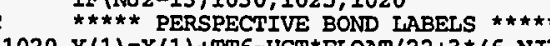

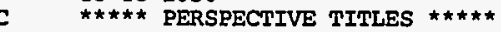

$1025 Y(1)=X(1)+T T 6-H G T * 215 . / 7$.

ILAST $=18$
$D X W=H G T * 24 . / 7$.

1030 GO TO 1050

$D V=H G T * .5$

$\mathrm{Y}(1)=\mathrm{Y}(1)-\mathrm{D} \mathrm{H}^{*} \mathrm{COSTH}+\mathrm{DV} * \mathrm{STNTH}$

$\mathrm{Y}(2)=\mathrm{Y}(2)-\mathrm{DH} \star \mathrm{SINTH}-\mathrm{DV} * \mathrm{COSTH}$

$Y(3)=0$.

$1050 \mathrm{Z}(3)=\mathrm{Y}(3)$

GO TO $(1060,1060,1060,1090,1090,1090,915,1105,1105)$, NJ3

1060 if (nj3 .eq. 1) go to 1061

$c^{* \star *}$ if title begins in column 1 , center it

if (title2(1)(1:1) .ne. 'i then

do $101 i=1,72=$.

101 continue

do $102 i=1,18$

tmpti $(i * 4-3: i * 4)=\operatorname{title} 2(i)$

102

do $103 \quad i=72,1,-1$ 


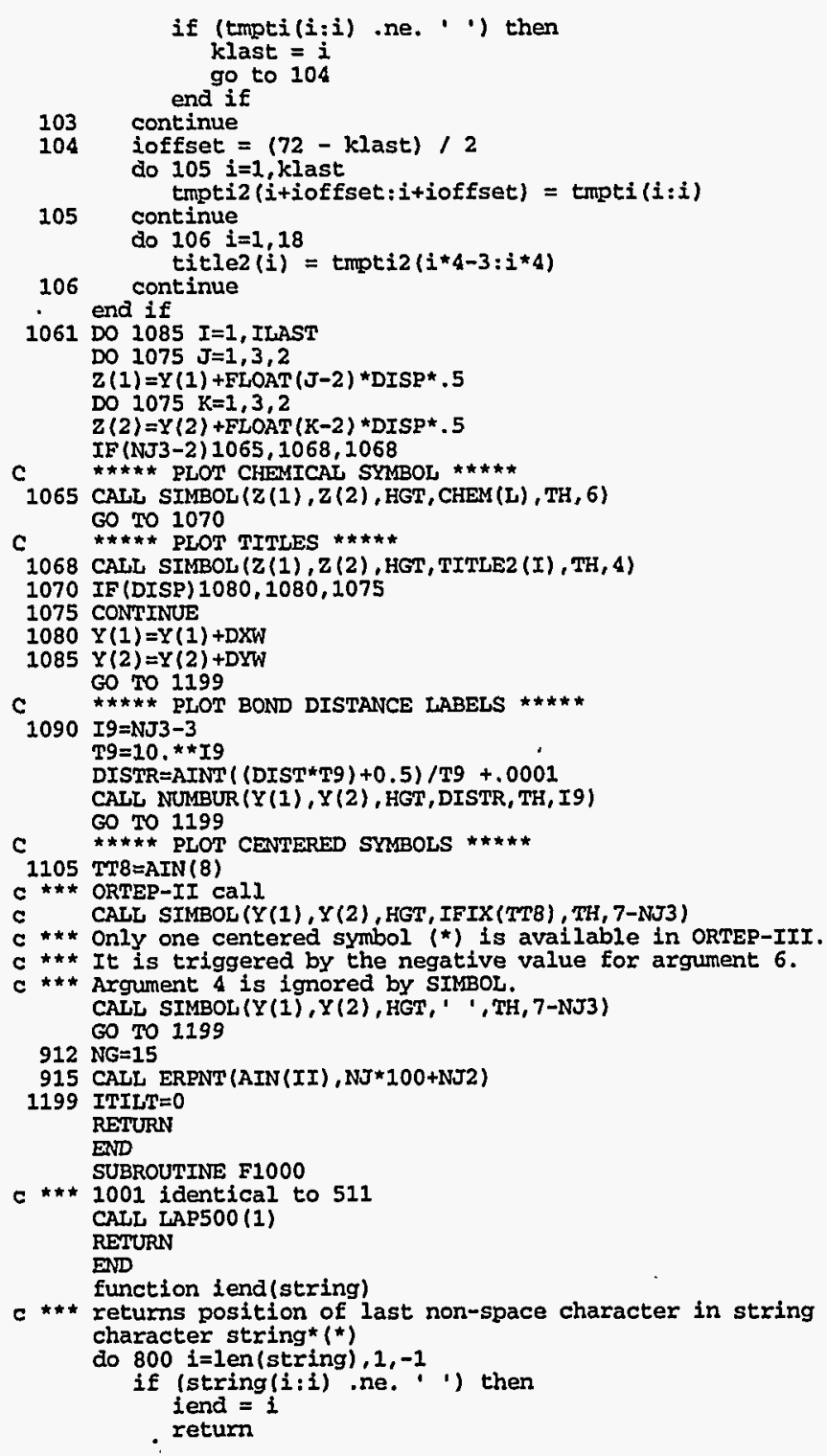

***** STORE PROTECTED ATOM CONICS AND BOND QUADRANGLES ***** DIMENSION $Q C(3,3), Q D(3,3), \operatorname{VD} 1(3), \operatorname{VD2}(3)$

COMMON/OLAP/CONIC (7, 500$),$ COVER $(6,20), \mathrm{KC}(20), \mathrm{KQ}(30)$, NCONIC, NCOVER COMMON/OLAP/CONIMT, REA $\star 8, A N U A D$, , ONAD CHARACTER * 4 TITLE, TITLEP COMMON NG, A $(9), \operatorname{AA}(3,3), \operatorname{AAREV}(3,3), \operatorname{AANRK}(3,3), \operatorname{AID}(3,3)$

$1, \operatorname{AIN}(140), \operatorname{ATOMID}(500), \operatorname{ATOMS}(3,500), \mathrm{BB}(3,3), \operatorname{BRDR}, \mathrm{CD}(8,20)$ $2, \operatorname{CONT}(5), \mathrm{D}(3,130), \mathrm{DA}(3,3), \mathrm{DP}(2,230), \mathrm{DISP}, \operatorname{EDGE}, \operatorname{FORE}, \operatorname{FS}(3,3,96)$

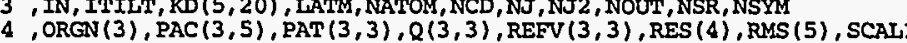
. 6 , VIEW, VT $(3,4), \mathrm{V1}(4), \mathrm{V} 2(3), \mathrm{V} 3(3), \mathrm{V} 4(3), \mathrm{V} 5(3), \mathrm{V} 6(3), \operatorname{WRKV}(3,3)$

$c$ * **** ELTMINATE ALI PREVIOUSLY STORED LOCAL OVERLAP INFORMATION ** NCOVER $=0$
NQOVVRR $=0$

IF (NTYPE) 420

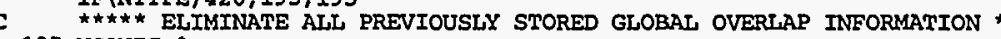
NCONIC $=0$

C IF (NTTYPE) 420,420, 200 .

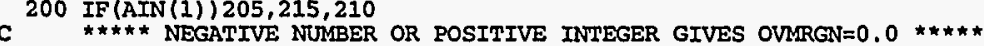

205 OVMRGN $=0.0$

GO TO 220

C 210 OVMRGN=AIN(1)-DINT(AIN(1))

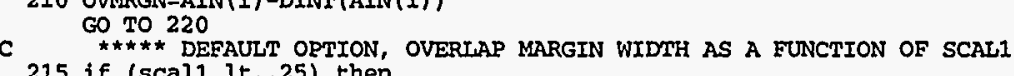
5 if (scall.1t..25) the

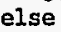

C GO TO 260 CARTESIAN COORDINATES IF VIEW.EQ.2ERO $* \star \star \star \star *$ 250 DO 255 I=1, LATI 
C ${ }^{* \star \star \star \star *}$ SORTING PROCEDURE BY SHELL, COMM ACM 2,30 (1959) $\star \star \star \star * *$ 260 M=LATM

IF (M) $300,300,270$

$70 \mathrm{~K}=\mathrm{LATMM}-\mathrm{M}$

$J=1$

$275 \quad I=J$

$280 \mathrm{IM}=\mathrm{I}+\mathrm{M}$

$\operatorname{IF}(\operatorname{ATOMS}(3, I)-\operatorname{ATOMS}(3, \operatorname{IM}))$ 295, 295, 285

285 TD=ATOMID $(I)$

ATOMID $(I)=$ ATOMID $(I M)$

ATOMID (IM) $=T$

$\operatorname{ATOMS}(3, I)=\operatorname{ATOMS}(3, I M)$

$\operatorname{ATOMS}(3, \mathrm{IM})=\mathrm{T} 1$

$I=I-M$

295 J $J+5+1295,295,280$

$\operatorname{IF}(J-K) 275,275,265$

C $\begin{gathered}\text { IF }(\mathrm{J}-\mathrm{K}) 275,275,265 \\ 300 \text { DO } 405 \text { LOP TARO THRGH ALI ATOMS IN SORTED ATOMS LIST } \star \star \star \star \star \star\end{gathered}$

CALLL XYZ (ATOMID (IA), ATOMS $(1, I A), 2)$

DO $305 \mathrm{~J}=1,3$

V1 (J) $=\operatorname{ATOMS}(J$, IA

DO $305 \mathrm{~K}=1,3$

$305 \mathrm{QD}(\mathrm{J}, \mathrm{K})=\mathrm{Q}(\mathrm{J}, \mathrm{K})$

IF (VIEW) $340,340,310$

CONE WITH ORIGIN AT VIEWPOINT

$310 \mathrm{~V} 1(3)=\mathrm{V} 1(3)-\mathrm{VIEW}$

(

$\mathrm{DO} 315 \mathrm{~J}=1,3$

$J 2=\operatorname{MOD}(\mathrm{J}+1,3)+1$

DO $315 \mathrm{~K}=\mathrm{J}, 3$

$\mathrm{K} 1=\mathrm{MOD}(\mathrm{K}, 3)+1$

$K 2=M O D(K+1,3)+1$
$Q C(J, K)=Q D(J 1, K 1) * Q D(J 2, K 2)-Q D(J 1, K 2) * Q D(J 2, K 1)$

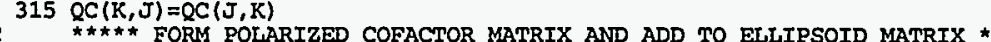

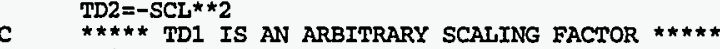

TD1 $=\mathrm{VMV}(\mathrm{V} 1, Q, \mathrm{~V} 1)$

DO $325 \mathrm{~J}=1,3$

$\mathrm{J} 2=\operatorname{MOD}(\mathrm{J}+1,3)+1$

$00320 \mathrm{~K}=\mathrm{J}, 3$

$\mathrm{K} 1=\operatorname{MOD}(\mathrm{K}, 3)+1$

$\mathrm{K} 2=\mathrm{MOD}(\mathrm{K}+1,3)+1$

$Q D(J, K)=((V D 1(J) *(0 C(J 1, K 1) *$

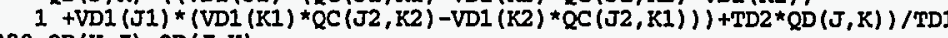

$320 \mathrm{QD}(\mathrm{K}, \mathrm{J})=Q D(\mathrm{~J}, \mathrm{~K})$

DITTPSE IN HOMOGENEOUS COORD OF WORKING SYSTEM ***

$\mathrm{QD}(\mathrm{J}, 3)=-\mathrm{QD}(\mathrm{J}, 3)$ *VIEW
$325 \mathrm{QD}(3, \mathrm{~J})=-\mathrm{QD}(3, \mathrm{~J}) * \mathrm{VIEW}$

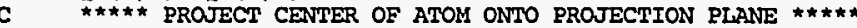

TD1 $=-V I E W / V D 1(3)$

$\mathrm{VD2}(1)=\operatorname{VD1}(1) * \mathrm{TD1}$
$\mathrm{VD2} 2(2)=\operatorname{VD} 1(2) * \mathrm{TD} 1$

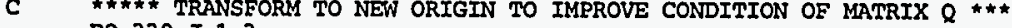
DO $330 \quad \mathrm{~J}=1,3$

$330 \mathrm{QD}(\mathrm{J}, 3)=\mathrm{QD}(\mathrm{J}, 3)+\mathrm{QD}(\mathrm{J}, \mathrm{K}) * \mathrm{VD} 2(\mathrm{~K})$

$\mathrm{D}(\mathrm{J}, 3)=Q D(\mathrm{~J}, 3)$
$\mathrm{D}=1,3$

DO $335 \mathrm{~K}=1,2$

$335 \mathrm{OD}(3, \mathrm{~J})=\mathrm{OD}(3, \mathrm{~J})+\mathrm{VD} 2(\mathrm{~K}) * \mathrm{OD}(\mathrm{K}, \mathrm{J})$

$\mathrm{V} 6(1)=\mathrm{XO}(1)+\mathrm{VD} 2(1)$
$\mathrm{V} 6(2)=\mathrm{XO}(2)+\mathrm{VD} 2(2)$

$\mathrm{V} 6(2)=\mathrm{XO}(2)+\mathrm{V}$
$\mathrm{GO}$ TO 355

***** CRICULATE ENVELOPING CYLINDER ALONG $\mathrm{z}$ OF WORKING SÝSTEM $* * *$

$340 \mathrm{DO} 345 \mathrm{J=1}, 2$

$345 \mathrm{QD}(\mathrm{J}, \mathrm{K})=\mathrm{QD}(\mathrm{J}, \mathrm{K})-\mathrm{QD}(\mathrm{J}, 3) * \mathrm{QD}(\mathrm{K}, 3) / \mathrm{QD}(3,3)$

DO $350 \mathrm{~J}=1,2$

$Q D(J, 3)=0.0$

$350 \mathrm{~V} 6(\mathrm{~J})=\mathrm{TO}(\mathrm{J})+$ ATOKS $(\mathrm{J}, \mathrm{TA}$

C $\$ \pi \hbar \star \star \pi$ PROJECTED ELIIPSE IN HOMOGENEOUS COORD ABOUT CENTER OF ATOM

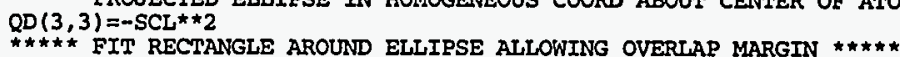

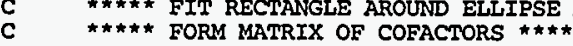

$355 \mathrm{DO} 360 \mathrm{~J}=1,3$

$\mathrm{J} 1=\operatorname{MOD}(\mathrm{J}, 3)+1$
$\mathrm{~J} 2=\operatorname{MOD}(\mathrm{J}+1,3)+1$

DO $360 \mathrm{~K}=\mathrm{J}, 3$

$360 \mathrm{QC}(\mathrm{J}, \mathrm{K})=\mathrm{QD}(\mathrm{J} 1, \mathrm{~K} 1) * \mathrm{OD}(\mathrm{J} 2, \mathrm{~K} 2)-\mathrm{OD}(\mathrm{J} 1, \mathrm{~K} 2) * \mathrm{OD}(\mathrm{J} 2, \mathrm{~K} 1)$

C DO $365 \mathrm{~J}=1,3$
DO $365 \mathrm{~K}=\mathrm{J}, 3$

$Q C(\mathrm{~J}, \mathrm{~K})=Q C(\mathrm{~J}, \mathrm{~K}) / Q \mathrm{C}(3,3)$

$365 \mathrm{QC}(\mathrm{K}, \mathrm{J})=\mathrm{QC}(\mathrm{J}, \mathrm{K})$

TD2 $=Q D(3)$

C DO $385 \mathrm{~J}=1,2$ 2 (

$\mathrm{T} 1=\mathrm{QC}(3, \mathrm{~J}) \star \star 2-\mathrm{QC}(\mathrm{J}, \mathrm{J})$

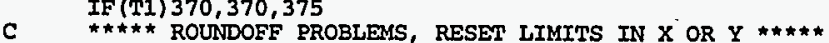

NDG=1

GO TO 380

375 V5 $(J)=\operatorname{SQRT}(T 1)+$ OVMRGN $\mathrm{V} 6(\mathrm{~J})=\mathrm{V} 6(\mathrm{~J})+\mathrm{QC}(3, \mathrm{~J})$

$380 \operatorname{CONIC}(2 * \mathrm{~J}-1, \mathrm{IA})=\mathrm{V} 6(\mathrm{~J})-\mathrm{V} 5(\mathrm{~J})$

380
$\operatorname{con}(\mathrm{N})$

IF (NDG) $390,390,395$

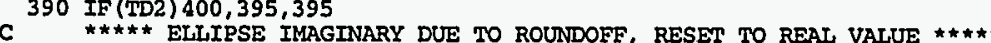

$95 \operatorname{CONIC}(5, I A)=1.0 /\left(\left(\operatorname{CONIC}\left(2, I_{A}\right)-\operatorname{CONIC}(1, I A)\right) * 0.5\right) \star \star 2$ $\operatorname{CONIC}(6, I A)=0.0$
$\operatorname{CONIC}(7, I A)=1.0 /(\operatorname{CONIC}(4, I A)-\operatorname{CONIC}(3, I A)) \star 0.5) * \star 2$ (1)

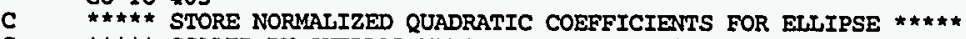

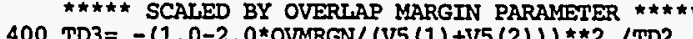
$\operatorname{CONIC~}(5, I A)=Q D(1,1) * T D 3$
$\operatorname{CONIC}(6, I A)=Q D(1,2) * T D 3$ 
$\operatorname{CONIC}(7, I A)=O D(2,2) \star T D 3$

CONTINUE

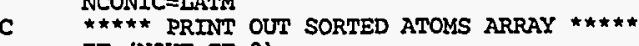

IF (NOUT.GE.0)

TOMTD ( $J), J=1$, LATM)

(1H0, 10X, 30HCONTENTS OF SORTED ATOMS ARRAY/(15X, 10F10.0))

C $\star * \star * *$ STORE BOND QUADRANGLS IF SEARCH CODES ARE GTVE *****

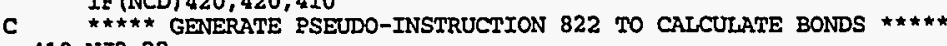

410 NJ2 $=22$

c *** the lines below have been moved to the end of F800

c c $\star * \star \star \star *$ PRINT OUT NUMMBER OF BOND OUADRANGLES STORED $* * \star * *$

c C $\star * \star \star *$ PRINT OUT QUADRANGLE IDENTIFICATION ARRAY $\star * \star * * *$

415 IF (NOUT.GE.0)

EWRITE (NOUT, 6) NQUAD, (QUAD $(9, J), J=1$, NQUAD)

6 FORMAT (1H0,10X,27HBOND OVERLAP ARRAY CONTAINS, I4, 23H BONDS (MAXIMU IM IS 599)/ 11X, 66HATOM-PAIR NUMBERS IN ARRAY REFER TO SEQUENCE

c 2TN 50 Th

$\star \star \star$ the lines above have been moved to the end of F800

20 RETUR

DIMENSION DETER $(2), Q A(3,3,2), Q C(3,3,2), \operatorname{V12}(3,2), \operatorname{VMIN}(2), \operatorname{MMAX}(2)$

REAI *8 AOV3, AOV3SQ, BOV3, DETER, PI, PHI, POV3, POV3CU, QA, QC, QOV2, QOV2SQ

, NCONIC, NCOVER,

NEAL*B AIN, ATOMID

CHARACTER * TITLE, TITLE2

COMMON NG, A $(9), \operatorname{AA}(3,3), \operatorname{AAREV}(3,3), \operatorname{AANRK}(3,3), \operatorname{AID}(3,3)$

1 ,AIN (140), ATO

(1)

, ORGN (3), PAC (3,5), PAT $(3,3), 0(3,3), \operatorname{REFV}(3,3), \operatorname{RES}(4), \operatorname{RMS}(5), \operatorname{SCAL}$

, SCAL2, SCL, SYMB $(3,3)$, TAPER, THETA, TITLE (18), TITLE2 $(18)$, TS $(3,96)$

6 , VIEW, VT $(3,4), \mathrm{V1}(4), \mathrm{V} 2(3), \mathrm{V} 3(3), \mathrm{V} 4(3), \mathrm{V} 5(3), \mathrm{V} 6(3), \operatorname{WRKV}(3,3)$

XING (3), Xo (3),XT(3)

$\mathrm{PI}=3.1415$
$\mathrm{ICQ}=0$

NCOVER $=0$

IF (NCONIC-NA) $200,200,20$

200 RETURN

ROUGH CHECK FOR OVERLAPPING ATOMS *****

DO $210 \mathrm{~J}=1,2$

YMAX (J) $=\operatorname{coNIC}(2 * J-1, N A)$

DO 420 IA $=\mathrm{NA}, \mathrm{NCONIC}$

IF (IA-NA) 230, 230,215

215 DO $225 \mathrm{~J}=1,2$

-CONTC $(2 * J-1, T A)) 420,420,220$

$220 \operatorname{IF}($ YMIN $(J)-\operatorname{CONIC}(2 * J, I A)) 225,420,420$

C 22 *
230 IF (L-1) 235, 235, 240

240 CALI LAPCON (CONIC (1, IA), DA, V12 $(1, \mathrm{~L})$, OVMR (L)

DO $245 \mathrm{~J}=1,3$

$245 Q A(U, K, L)=D A(J, k$

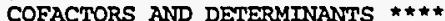

$\operatorname{DETER}(L)=0.0$

$\mathrm{JI}=\mathrm{MOD}(\mathrm{J}+3,3)+1$

$J 2=\operatorname{MOD}(\mathrm{J}+1,3)+1$

DO $250 \mathrm{~K}=1,3$

$\mathrm{KI}=\mathrm{MOD}(\mathrm{K}+3,3)+$

$\mathrm{TD}=\mathrm{QA}(\mathrm{J} 1, \mathrm{~K} 1, \mathrm{~L}) * \mathrm{QA}(\mathrm{J} 2, \mathrm{~K} 2, \mathrm{~L})-\mathrm{QA}(\mathrm{J} 1, \mathrm{~K} 2, \mathrm{~L}) * \mathrm{QA}(\mathrm{J} 2, \mathrm{~K} 1, \mathrm{~L})$

DETER $(L)=D E T E R(L)+T D * Q A(J, K, L)$

$250 Q C(J, K, \mathrm{I})=T D$

IS THE DETERMINANT TIMES $3 \star \star \star \star \star *$

IF (I, -1 ) $420,420,255$

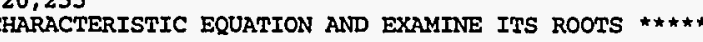

AOV $3=0.0$

DO $260 \quad \mathrm{~J}=1,3$

AOV $3=\mathrm{AOV} 3+\mathrm{QC}(\mathrm{J}, \mathrm{K}, \mathrm{S}) * \mathrm{QA}\left(\mathrm{J}, \mathrm{x}_{1}\right.$

260 BOV $3=B O V 3+Q C(J, K, 1) * Q A(J, K, 2)$

AOV $3=A O V 3 /$ DETER $(2)$

BOV $=$ BOV 3 / DETER

POV $3=$ BOV $3-2 O V 3 S P(2)$

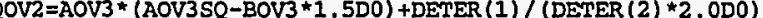

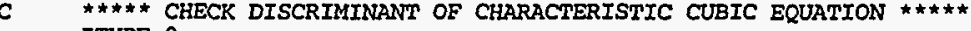

ITYPE $=0$

POV3CU $=$ POV $3 * * 3$

IF (POV3CU+QOV2SQ) 270,310,265

265 IF (POV3CU*1.00001 +QOV2SQ) 310,310,400

270 IF (POV3CU+1.00001 *COV2SQ) 275,310,310

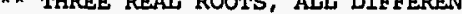

275 ITYPE $=1$

$\star \star \star \star \star$ NO INTERSECTION IF $A / 3$ AND B/3 INVARIANTS ARE NDGATIVE $* \star \star \star *$ IF (AOV3) $280,285,285$

C $* * \star * \star$ CALCULATE ONE ROOT OF CHARACTERISTIC CUBIC EQUATION $* * * * *$

285 IF (QOV2) $295,290,295$

$290 \mathrm{PHI}=\mathrm{PI} / 2 . \mathrm{OD}$

295 PHI=DATAN (-DSQRT (-POV3CU-QOV2SQ) /QOV2)

IF (PHI) 300, 305, 305

300 PHI $=$ PHI + PI

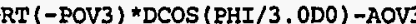

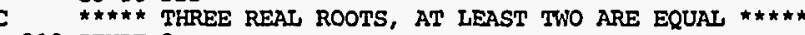

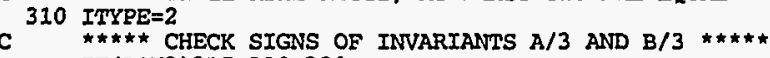

315 IF (AOV) $315,320,320$

C $* \star * \star *$ CALCULATE REPEATED ROOT OF CUBIC EQUATION $* \star \star * *$

320 ROOT=DSIGN (DSQRT (-POV3), OOV2) -AOV3

C $3 * * *$ FORM DEGENERATE CONIC (LINE PAIR WHICH MAY BE COINCIDENT) * 
DO $330 \mathrm{~K}=1,3$

(3)

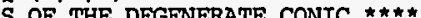
$T 6=\mathrm{DA}(1,1) * \mathrm{DA}(2,2)$

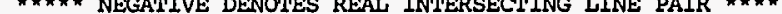

***** POSITIVE DENOTES IMAGINARY LINES INTERSECTING AT REAL POINT IF (T6-T7) $335,345,340$

335 IF $(26 * 1.0011-T 7) 400,345,345$

345 TF=DA $(3,3) *(2,1,1)+0,342,365$

$T 9=D A(1,3) \star \star 2+D A(2,3) \star \star 2$

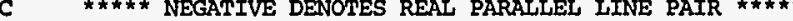

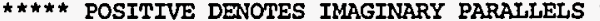

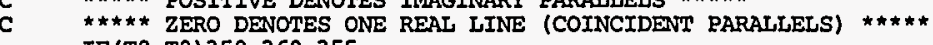
IF (T8-T9) $350,360,355$

$350 \mathrm{IF}(\mathrm{T} 8 * 1.0001-\mathrm{T} 9) 400,360,360$

c $\star \star \star \star \star$ COINCIDENT LINE PAIR FOUND FOR THE REPEATED ROOT $\star \star \star \star \star ~$

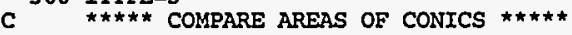

$365 \mathrm{KA}=1$

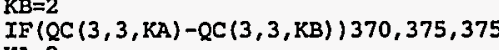

370 KA

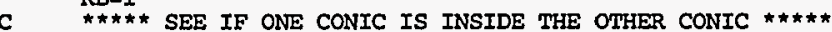

$375 \mathrm{~T} 1=0.0$

DO $385 \mathrm{~J}=1,3$

$\mathrm{T} 2=\mathrm{QA}(\mathrm{J}, 3, \mathrm{~KB})$

$\mathrm{D} 380 \mathrm{~K}=1,2$

$\star V 12(K, K A)$

C $* \star \star \star \star$ DISCARD IF KA IS OUTSIDE KB $* \star * * *$

IF (T1) $390,390,420$

(

395 ICQ $=-1$

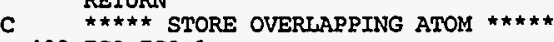

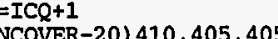

$405 \mathrm{NG}=17$

CALL ERENT (ATOMID (IA), 700)

410 NCOVER $=$ NCOVER +1

DO $415 \quad I=1,3$

COVER (IJ, NCOVER) $=Q A(I, J, 2)$

$415 \mathrm{IJ}=\mathrm{IJ}+1$

KC (NCOVER) $=$ IA

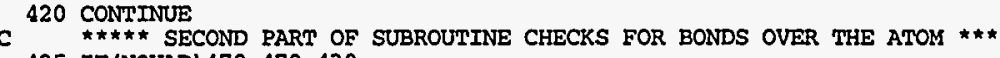
425 IF (NQUAD) $470,470,430$

C 430 ITY=0

DO 465 IQ $=1$, NQUAD
TID $=Q U A D(9, I Q)$

TIDD $=$ TID

$\mathrm{NA} 1=\mathrm{TID} / 1000$.
NA2 $=$ AMOD (TID, 1000.)

135 IF (NA-NA2) 435, 435, 465

IF (YMAX (J)-AMTN1 (QUAD (J,IQ), QUAD (J+2,IQ), QUAD (J+4,IQ), QUAD (J+6, IQ) (J) - MMAX1 (QUAD (J,IQ), QUAD (J+2,IQ), QUAD (J+4,IO), OUAD (J+6, IQ

$1) 445,465,465$

445 CONTINUE

(1)

$I Q Q=0$

CALL LAPAB (IOR, NA, IOQ, ITY)

$I C Q=-1$
RETURN

460 ICQ $=$ ICQ +1

$-30) 465,470,470$

465 CONTINUE
470 RETURN

END

SUBROUTTNE LAP800 (NA1, NA2, ICQ)

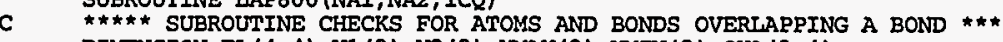
DIMENSION FL $(4,4), Y 1(2), Y 2(2), \operatorname{YMAX}(2), \operatorname{YMIN}(2), Q U A(3,4)$

DIMENSION VUE (3)

COMMON/OLAP/CONIC $(7,500)$, COVER $(6,20), \mathrm{KC}(20), \mathrm{KO}(30)$, NCONIC, NCOVER,

1 NOOVER, NOUAD, OVMRGN, QOVER $(3,4,30)$, OUAD $(9,600)$, SEGM $(50,2)$

REAL $\star 8$ ATN, ATOMID

CHARACTER * TITLE, TITLE2

COMMON NG, A (9), AA $(3,3), \operatorname{AAREV}(3,3), \operatorname{AAWRK}(3,3), \operatorname{AID}(3,3)$

$1, \operatorname{ArN}(140), \operatorname{ATOMID}(500), \operatorname{ATOMS}(3,500), \mathrm{BB}(3,3), \operatorname{BRDR}, \mathrm{CD}(8,20)$

E, ES $(3,3,96)$

$4, \operatorname{ORGN}(3), \operatorname{PAC}(3,5), \operatorname{PAT}(3,3), 0(3,3), \operatorname{REFV}(3,3), \operatorname{RES}(4), \operatorname{RMS}(5), \mathrm{SCAL} 1$

5 , SCAL2, SCL, SXMB $(3,3)$, TAPER, THETA, TITLE (18), TITLE2 (18) TS $(3,96)$

6 , VIEW, VT $(3,4), \mathrm{V} 1(4), \mathrm{V} 2(3), \mathrm{V} 3(3), \mathrm{V} 4(3), \mathrm{V} 5(3), \mathrm{V} 6(3), \mathrm{WRKV}(3,3)$

$7, X \mathrm{IING}(3), \mathrm{XO}(3), \mathrm{XT}(3)$

$I Q=0$

IF (NA1*NA2) 245, 245, 195

195 TID1 $=$ FLOAT (NA1) *1000.+FLOAT (NA2)

IF (NCONIC) 245, 245, 200

C 200 IF (NJ2 - 21) 250,205,205 205 IF (NQUAD-599) 215, 210,210

ERPNT (ATOMID (NA1) , 822)

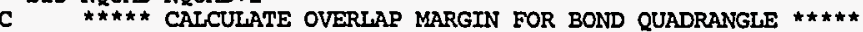

$\mathrm{T} 1=0.0$

$\mathrm{T} 2=0.0$

$D 0220 \mathrm{~J}=1,2$

$Y 1(J)=D P(J, 1)-D P(J, 65)$

$\mathrm{T} 1=\mathrm{T} 1+\mathrm{Y} 1(\mathrm{~J}) * \star 2$

$220 \mathrm{~T} 2=\mathrm{T} 2+\mathrm{Y} 2(\mathrm{~J}) * * 2$

$\operatorname{IF}(T 1 * T 2) 225,225,230$

$\begin{aligned} \mathrm{T} 1 & =0.0 \\ \mathrm{~T} 2 & =0.0\end{aligned}$ 
GO TO 235

$T 1=O$ YMRGN $/$ SQRT (TI)

D**** STORE BOND OUADRANGLE *****

235 DO 240 J $=1,2$

$\mathrm{YI}(\mathrm{J})=\mathrm{Y1}(\mathrm{J}) * \mathrm{~T}_{1}$

QUAD $(J, N Q U A D)=D P(J, 1)+\mathrm{Y} 1(\mathrm{~J})$

QUAD $(J+5, N Q U A D)=D P(J, 5)+Y 5(J)$

QUAD $(J+4, N Q U A D)=D P(J, 66)-Y 2(J)$

QUAD $(J+4, N, N)=D P(J, 6)$

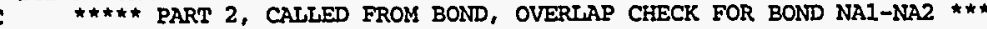

250 NCOVER $=0$

NQOVER $=0$

(1)

( $* * \star \star \star$ SAVE QUADRANGLE TEMPORARILY $* * * \star *$

255 IQ $=$ NQUAD +1

$\operatorname{QUAD}(J, I Q)=D P(J, 1)$

$\operatorname{QUAD}(J+2, I Q)=D P(J, 2)$

$(\mathrm{J}, 65$

QUAD $(9, I Q)=T$ T

265 DO $270 \mathrm{~J}=1,2$

$770 \operatorname{YMIN}(J)=\operatorname{AMINI}(\mathrm{DP}(J, 1), \mathrm{DP}(J, 2), \mathrm{DP}(J, 66), \mathrm{DP}(J, 65))$

$270 \operatorname{YMAX}(J)=A M A X 1$ (DP $(J, 1), D P(J, 2), D P(J, 66), D P(J, 65))$ NAI $\mathrm{P} 1=\mathrm{NA1}+1$

DO 305 IA=NA1P1, NCONIC

IF (IA-NA2) 275, 305, 275

(

285 CONTINUE

CONTINUE

ITY $=$ ITYY

CALL IAPAB (IO, IAQ, IQQ, ITY)

IF (IQQ) 290, 305, 300

$300 \mathrm{ICQ}=I \mathrm{CQ}+1$

IF (NCOVER-20) 305, 310,310

IF (NQUAD) 295, 295, 315

290 ICQ $=-1$

295 RETURN

315 CALL DIFV (ATOMS (1, NA2), ATOMS (1, NA1), V1)

CALL UNITY (V1,V1,1)

$\operatorname{VUE}(1)=\operatorname{ATOMS}(1, \mathrm{NA} 1)$

VUE $(3)=$ ATOMS $(3$, NA1) -VIEN

DO 495 IB $=1$, NQUAD

$\operatorname{TID} 2=$ QUAD $(9$, IB $)$

5,320

320 NB2=AMOD (TID2, 1000.)
NB1 $=$ TID $2 / 1000$

IP(4)

IF (YMAXX (J) -AMTN1 (QUAD (J, IB), QUAD (J+2, IB), QUAD (J+4, IB), QUAD (J+6, IB) $1) 495,495,330$ (Q $1) 1335,495,495$

335 CONTINUS

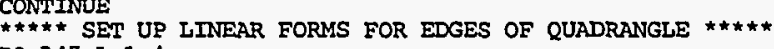

DO $345 \mathrm{~L}=1,4$

$\mathrm{K} 1=\mathrm{MOD}(\mathrm{K}, 8)+2$

OUA $(1, L)=$ OUAD $(K, I B)$ - OUAD (K1, IB)

QUA $(2, \mathrm{~L})=\mathrm{QUAD}(\mathrm{K} 1-1, \mathrm{IB})-\mathrm{QUAD}(\mathrm{K}-1, \mathrm{IB})$

QUA $(3, L)=Q U A D(K-1, I B) * Q U A D(K 1, I B)-Q U A D(K, I B) * Q U A D(K 1-1, I B)$

C $* \star * \star *$ NORMALIZE LINE EQUATION COEFFICIENTS $* \star \star \star * *$

$\mathrm{T} 1=\mathrm{SQRT}$ (QUA (1,

$340 \mathrm{DO} 345 \mathrm{~J}=1,3$

345 OU 10

C $* \star \star \star *$ EVALUATE LINEAR FORMS AND SIGNATURES FOR QUADRANGLE $\star \star \star * \star *$

$\mathrm{T} 3=3.0$

DO $365 \mathrm{~K}=1,4$

$\mathrm{T} 2=3.0$

Do $355 \quad L=1,4$

T1 $=$ QUAD $(J-1, I Q) * Q U A(1, L)+Q U A D(J, I O) * Q U A(2, L)+Q U A(3, L)$

(2) $=12-1.0$

IF (T2) $360,365,365$

365 CONTINUE

c ***** CHECK FOR 4 POINTS INSIDE QUADRANGLE *****

IF (T3) $370,375,375$

370 ITYPE $=-1$

C $* * \star \star \star$ CHECK FOR 2 TO 3 POINTS INSIDE QUADRANGLE ****

$375 \operatorname{IF}(T 3-3.0) 380,385,385$

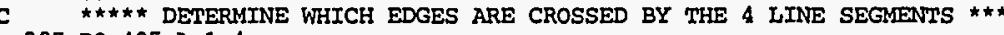

385 DO $405 \mathrm{~L}=1,4$

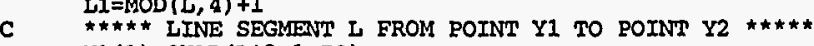

$Y 1(1)=Q U A D(L \star 2-1, I Q)$

Y $2(1)=Q U A D(L 1 * 2-1, T Q)$

$\mathrm{Y} 2(2)=\mathrm{QUAD}(\mathrm{L} 1 * 2, \mathrm{IO})$

DO $405 \mathrm{~K}=1,4$

$\mathrm{T} 1=\mathrm{FL}(\mathrm{K}, \mathrm{L})$

$\mathrm{T} 2=\mathrm{FL}(\mathrm{K}, \mathrm{L}, \mathrm{L})$

C $* \star * \star$ T1 AND T2 MUST HAVE OPPOSITE SIGNS FOR INTERSECTION TO $\propto$ CCUR IF (T1*T2) 390,390,405

$390 \mathrm{IF}(\mathrm{ABS}(\mathrm{T3})-1 . \mathrm{E}-5) 405,405,395$

$395 T 4=(T 1 * Y 2(1)-T 2 * Y 1$ (1) ) /T3

$T 5=(T 1 * 22$
$K 0=2 \star K$ 
$K 1=2 *(M O D(K, 4)+1)$

$\begin{array}{ll} & \\ & \end{array}$

$T 6=(T 4-Q U A D(K O-1, I B))$ * $(Q U A D(K 1-1, I B)-T 4)+(T 5-Q U A D(K O, I B))$ *

(QUAD (K1, IB) $-\mathrm{T} 5$ )

作

400 IF (T6) 405,410,410

GO TO 495

410 ITYPE=1

$415 \operatorname{IF}((\mathrm{NA1}-\mathrm{NB1}) *(\mathrm{NA2} 2 \mathrm{NB2}) *(\mathrm{NA2}-\mathrm{NB1})) 425,420,425$

420 IF (NA1+NA2-NB1-NB2) 465,495,495

425 CALL DIFV (ATOMS $(1, \mathrm{NB} 2)$, ATOMS $(1, \mathrm{NB} 1), \mathrm{V} 2)$

CALL UNITY (V2, V2, 1 )

CALL UNAT (VI, $V 4,1)$

$\operatorname{IF}(\mathrm{VW}$ (V3,V3)-TOL) $430,430,435$

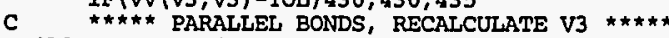

30 CALL NORM (V1, V4, V5, 1)

C $* \star * * \star$ CHECK FOR COLLINEAR BONDS $* * \star * \star *$

IF (VV (V3,V3) $-\mathrm{TOL}) 465,465,450$

440 DO $445 \mathrm{~J}=1,3$

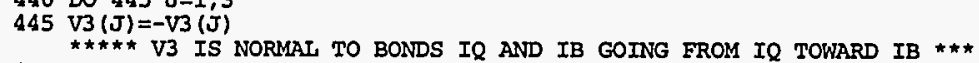

$450 \operatorname{IF}$ (VIEW) $455,455,460$

455 IF (V3 (3)) $495,495,465$

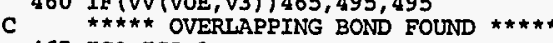

$465 I C Q=I C Q+1$

IF (ITYPE) 470, 475, 475

470 ICQ $=-1$

$$
\text { C RETURN }
$$

475 IF (NQOVER - 30) $485,480,480$

$$
\text { TIDD }=\text { TID2 }
$$

$$
\text { TIDD=TID2 }
$$

RETURN

$$
\text { NQOVER }=\text { NQOVER+1 }
$$

DO $490 \mathrm{~K}=1,4$

$490 \operatorname{COVER}(J, K, N Q O V E R)=Q U A(J, K)$

KQ (NQOVER) $=I B$

495 CONTINUE

500 REIURA

SUBROUTINE LAPAB (IQ, IA, ICQ, ITTY)

C $\star \star \star \star \star$ SUBROUTINE CHECKS FOR OVERLAP BETWEEN ATOMS AND BONDS $* \star \star * \star$ DIMENSION BF (4), CON $(3,3), O F(5)$, OUA $(3,4)$

COMMON/OLAP/CONIC $(7,500)$, COVER $(6,20), K C(20), K O(30)$, NCONIC, NCOVER 1 NQOVER, NQUAD, OVMRGN, $\operatorname{CVER}(3,4,30)$, QUAD $(9,600), S D G M(50,2)$ REAL*8 TIDD

REAL*8 AIN, ATOMID

COMMON NG, $A(9), \operatorname{AA}(3,3), \operatorname{AAREV}(3,3), \operatorname{AAWRK}(3,3), \operatorname{AID}(3,3)$
$1, \operatorname{AIN}(140), \operatorname{ATOMID}(500), \operatorname{ATOMS}(3,500), \operatorname{BB}(3,3), \operatorname{BRDR}, \operatorname{CD}(8,20)$

$2, \operatorname{CONT}(5), \mathrm{D}(3,130), \mathrm{DA}(3,3), \mathrm{DP}(2,130), \mathrm{DISP}, \operatorname{EDGE}, \operatorname{FORE}, \operatorname{FS}(3,3,96)$

$4, \operatorname{ORGN}(3), \operatorname{PAC}(3,5), \operatorname{PAT}(3,3), Q(3,3), \operatorname{REFV}(3,3), \operatorname{RES}(4), \operatorname{RMS}(5), \operatorname{SCAT}$

5 SCAL2, SCL, SYMB (3,3), TAPER, THETA TTTLE (18) TITI (18) (18) TS $(3,96)$

$6, \operatorname{VIEN}, \operatorname{VT}(3,4), \mathrm{V} 1(4), \mathrm{V} 2(3), \mathrm{V} 3(3), \mathrm{V} 4(3), \mathrm{V} 5(3), \mathrm{V} 6(3), \mathrm{WRKV}(3,3)$

TID $=$ QUAD $(9, \mathrm{IQ})$

$\mathrm{NA} 1=\mathrm{TID} / 1000$.

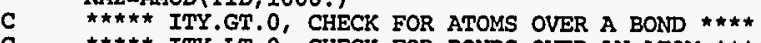

$$
I C Q=0
$$

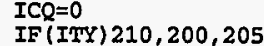

205 CALL LAPCON (CONIC (1, IA), CON, V1, 0.0)

IF (ITY-2) 220,240,240

215 CALI LAPCON (CONIC ( 1 , IA), CON, V1, OVMRGN)

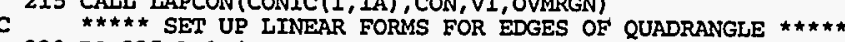

220 DO $235 \mathrm{~L}=1,4$

$\mathrm{K}=2 \star \mathrm{L}$
$\mathrm{K} 1=\mathrm{MOD}(K, 8)+2$

QUA $(1, L)=$ QUAD $(K, I Q)$-QUAD (K1, IQ

QUA $(2, I)=$ QUAD $(K 1-1, I Q)-$ QUAD $(K-1, I Q)$

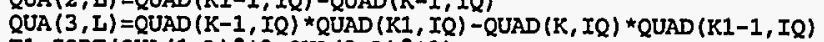

IF (TI) $225,225,230$

225 ITY $=0$

ICQ $=0$

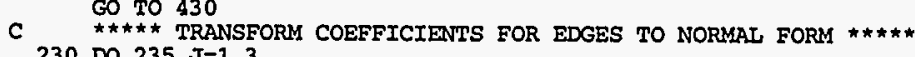

$230 \mathrm{DO} 235 \mathrm{~J}=1,3$

***** EVALUATE 4 QUADRATIC AND 4 BILINEAR FORMS $* \star \star * *$

V2 $(3)=1.0$
V3 $(3)=1.0$

$\mathrm{T} 2=3.0$

DO $265 \mathrm{~L}=1,4$

$\mathrm{LI}=(\operatorname{MOD}(\mathrm{L}, 4)+1) \star 2$

$\mathrm{V} 2(1)=Q U A D(2 * \mathrm{~L}-1, \mathrm{IQ})$

V $3(1)=$ OUAD $(L 1-1, I Q)$

V3 $(2)=Q U A D(L 1, I Q)$

$Q E(L)=0.0$

$\mathrm{BF}(\mathrm{L})=0.0$

DO $250 \mathrm{~K}=1,3$

$T=\operatorname{CON}(3, K)$

$245 \mathrm{~T} 1=\mathrm{T} 1+\mathrm{V} 2(\mathrm{~J}) * \operatorname{CON}(\mathrm{J}, \mathrm{K})$

$250 \mathrm{QF}(\mathrm{L})=\mathrm{QF}(\mathrm{L})+\mathrm{T} 1 * \mathrm{~V} 2(\mathrm{~K})$

$\mathrm{BF}(\mathrm{L})=\mathrm{BF}(\mathrm{L})+\mathrm{T} 1 * \mathrm{~V} 3(\mathrm{~K})$
$\mathrm{IF}(\mathrm{QF}(\mathrm{L})) 260,255,265$

$255 \mathrm{~T} 2=\mathrm{T} 2-0.8$

$60 \mathrm{~T} 2=\mathrm{T} 2-1.0$

$260 \mathrm{~T} 2=\mathrm{T} 2-1.0$

C $\star \star \star \star \star$ CHECK FOR 4 POINTS OF QUADRANGLE INSIDE OR ON ELLIPSE $\star \star \star \star \star ~$ IF (T2) $270,275,275$ 
$\quad$ GO TO 330

FOR 1 TO 3 POINTS OF QUADRANGLE INSIDE THE ELLIPSE $* \star \star$ 275 IF (T2-2.2)

IF (NA2-IA) $340,375,335$

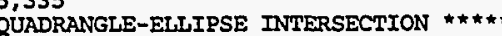

C 285 DO 305 K=1,4

$T 1=B F(K) * * 2-Q F(K) * Q F(K+1)$

IF (T1) $305,305,290$

$290 \mathrm{~T} 1=\mathrm{SQRT}(\mathrm{TI})$

C $\star \star \star \star \star$ IS INTERSECTION WITHIN BOUNDS OF QUADRANGLE $* \star \star \star *$ $T 3=Q E(K)-B F(K)$

$T=T B(T)-1 . E-5) 305,305,295$

295 T5=(T3-T1)/T

IF (T5) $305,280,300$

$300 \operatorname{IF}(1.0-T 5) 305,305,280$

305 CONTINUE

SECTION FOIND $* * * * *$

a

$$
T 3=3.0
$$$$
\text { DO } 320 K=1,4
$$

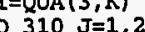

$310 \mathrm{Tl}=\mathrm{T} 1+\mathrm{V} 1(\mathrm{~J}) * \mathrm{QUA}(\mathrm{J}, \mathrm{K})$

IF (T1) $315,320,320$

$315 T 3=T 3-1.0$

IF (T3) $325,370,370$

325 ITYPE $=1$

法

330 IF (NA2-IA) $375,375,335$

335 IF (IA-NA1) $375,375,340$ (1)

CALL DIFV ATOMS $(1$, NA2), ATOMS (1, NA1), V2)

CALL DUNTY (V2, V2, IA)

CALL UNITYY (V3, $V 3,1$

CALL NORM(V2, V3, V4 1 )

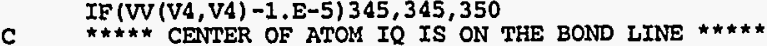

345 IF(ITY) $370,370,385$ IS

350 CALS NORM (V4, V2, V5, 1 )

$\mathrm{T} 1=-\mathrm{V} 5(3)$

$355 \mathrm{TI}=\mathrm{VS}(3) \star(A T O M S(3, \mathrm{IA})-\mathrm{VIEW})$

DO $360 \mathrm{~J}=1,2$

$360 \mathrm{~T} 1=\mathrm{T} 1+\mathrm{V} 5$ (J) *ATOMS (J, IA)

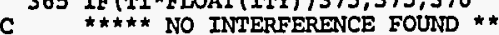

GO TO 430

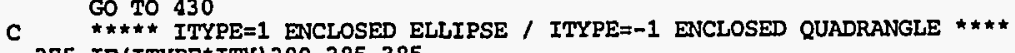

375 IF (ITYPE*ITY) $380,385,385$

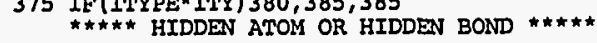

GO TO 430

385 ICQ $=1$

C IF(ITY) $410,390,390$
390 IF (NCOVER-20) 400,395,395

395 NG $=17$

CALI ERPNT (ATOMID (IA), 800)

NCOVER $=$ NCOVER -1

DO $405 \quad I=1,3$

COVER $(I J, N C O V E R)=\operatorname{CON}(I, J)$

405 IJ $=I J+1$

CO TO 430 .

410 IF (NOOVER-30) 420,415, 415

$\mathrm{NG}=18$

$$
\text { TIDD = TID }
$$

NQOVER $=$ NQOVER -1

420 NQOVER $=$ NQOVER

DO $425 \mathrm{~K}=1,4$

$425 \operatorname{QOVER}(J, K$, NQOVER $)=Q U A(J, K)$

$\mathrm{KQ}$ (NQOVER) $=$ IQ

430 RETURN
SUBROUTINE LAPCON (CON1, CON, Y, OVMR)

HOMOGENEOUS COORDINATE SYSTEM $\star \star \star ~$ DIMENSION CONI (7), CON $(3,3), Y(3)$

$\mathrm{Y}(1)=(\operatorname{CON} 1(1)+\operatorname{CON} 1(2)) * 0.5$

$x(2)=(\operatorname{CoN} 1(3)+\operatorname{CoN} 1(4)) * 0$.

$Y(3)=1.0$

$\operatorname{CoN}(1,1)=\operatorname{CON} 1(5)$

$\operatorname{CON}(1,2)=\operatorname{CON}(6)$

$\operatorname{CoN}(2,2)=\operatorname{CON} 1(7)$

$T 1=(\operatorname{CoN} 1(2)-\operatorname{CoN} 1(1)+\operatorname{CON} 1(4)-\operatorname{CoN} 1(3)) * 0.25$

$\operatorname{CON}(3,3)=-((T 1-O V M R) / T 1) \star \star 2$

DO $205 \mathrm{~K}=1,2$

$\cos (K, 3)=0.0$

$200 \operatorname{CON}(K, 3)=\operatorname{CON}(K, 3)-Y(J) * \operatorname{CON}(J, K)$

$205 \operatorname{CoN}(3, K)=\operatorname{CON}(K, 3)-Y(J) * \operatorname{con}(J, K)$

REIURN

END

SUBROUTINE LAAPDRW (Y, NPEN, NCQ)

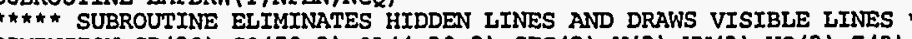
DIMENSTON

1 NQOVER, NQUAD, OVMRGN, QOVER $(3,4,30), \operatorname{QUAD}(9,600), \operatorname{SEGM}(50,2)$

REAL $* 8$ AIN, ATOMID

CHARACTER $* 4$ TITLE, TITLE2

COMMON NG, $A(9), \operatorname{AA}(3,3), \operatorname{AAREV}(3,3), \operatorname{AANRK}(3,3), \operatorname{AID}(3,3)$

2 AIN (140), ATOMID (500), ATOMS $(3,500), \operatorname{BB}(3,3), \operatorname{BRDR}, \operatorname{CD}(8,20)$

3 ,'IN, ITILT, KD $(5,20)$, LATM, NATOM, NCD, NJ, NJ2, NOUT, NSR, NSYM 3,96$)$

4 , ORGN (3), PAC (3,5), PAT $(3,3), Q(3,3), \operatorname{REEV}(3,3), \operatorname{RES}(4), \operatorname{RMS}(5)$, SCAL,

$5, \operatorname{SCAL} 2$ SCL, SXMB $(3,3)$, TAPER, THETA, TITLE (18), TITLE2 (18) TS $(3,96)$

$6, \operatorname{VIEW}, \operatorname{VT}(3,4), \mathrm{V} 2(4), \operatorname{V2}(3), \operatorname{V3}(3), \mathrm{V} 4(3), \mathrm{V} 5(3), \mathrm{V} 6(3), \operatorname{WRKV}(3,3)$

7 ,XING (3), XO(3),XT(3) 
$\mathrm{NCQ}=\mathrm{NCOVER}+\mathrm{NQOVER}$

IF (NCQ) 200,200,205

(200 IF(NPM3) $210,230,230$

C

10 YO (1) $=$ YN (1)

$\mathrm{YO}(2)=\mathrm{YN}(2)$
$\mathrm{YO}(3)=1.0$

$Y O(3)=1$.
NPO $=$ NPN

DO $215 \mathrm{~K}=1, \mathrm{NCO}$

IF (NOOVER) $230,230,220$

220 DO $225 \mathrm{~K}=1$, NQOVER

$225 \mathrm{QL}(\mathrm{J}, \mathrm{K}, 1)=\mathrm{QL}(\mathrm{J}, \mathrm{K}, 2)$

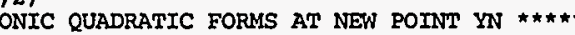

YN(1) $=\mathrm{Y}(1)$

YN $(2)=Y(2)$
YN $(3)=1.0$

NPN $=$ NPEN

IF (NCOVER) 250,250,235

235 DO $245 \mathrm{~K}=1$, NCOVER

(1) $=\mathrm{YN}(1) * \operatorname{COVER}(1, \mathrm{~K})+\mathrm{YN}(2) * \operatorname{COVER}(2, K)+\operatorname{COVER}(3, \mathrm{~K})$

$2(2)=$ N $(1) * C O V E R(1, K)+Y N(2) * C O V E R(2, K)+C O V E R(3, x)$

$C O(K, 2)=Z(1) * Y N(1)+Z(2) *$ KN $(2)+Z(3)$

c $\star \star \star \star \star$ EVALUATE CONIC BILINEAR FORM IF PEN IS DOWN $* * \star * *$ IF (NPM3) 240,245,245

$240 \mathrm{CB}(\mathrm{K})=\mathrm{Z}(1)$ *YO $(1)+\mathrm{Z}(2) * \mathrm{YO}(2)+\mathrm{Z}(3)$

245 CONTINUE

250 IF (NOOVER) 275, 275,255

NOEOVER

$\mathrm{T} 2=3.0$

DO $265 \mathrm{~J}=1,4$

(1, J,K)+YN $(2) * Q$ OVER $(2, J, K)+Q$ OVER $(3, J, K)$

IF (T1) $260,265,265$

$260 \mathrm{~T} 2=\mathrm{T} 2-1.0$

$\mathrm{KCQ}=\mathrm{KCQ}+1$

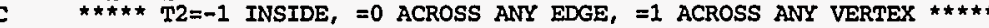

$270 \mathrm{CQ}(\mathrm{KCQ}, 2)=\mathrm{T2}$

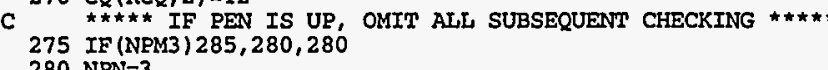

CALI SCRIBE (YN, NPN)

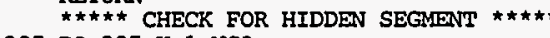

$285 \mathrm{DO} 295 \mathrm{~K}=1$, NCO

$290 \operatorname{IF}(C Q(K, 1)) 290,295,295$

CONTINUE

$N I N T=0$

IF (NCOVER) $330,330,300$

$300 \mathrm{DO} 325 \mathrm{~K}=1$, NCOVER

$T 1=C B(K) \star \star 2-C Q(K, 1) * C Q(K, 2)$
IF (T1) $325,325,305$

$305 \mathrm{~T} 1=\mathrm{SQRT}$ (TI)

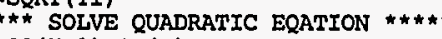

$\mathrm{T} 2=\mathrm{CQ}(\mathrm{K}, 1)-\mathrm{CB}(\mathrm{K})$

TF(ABS (T3) $-1, E-5) 325,325,310$

$310 T 4=(T 2-T 1) / T 3$

$\mathrm{T} 5=(\mathrm{T} 2+\mathrm{T} 1) / \mathrm{T} 3$

**** VALID INTERSECTION IF T4.LT.1 AND T5.GT.0 *****

IF (T4-1.0) $315,325,32$

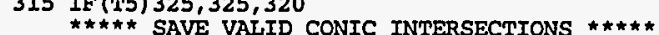

SEGM (NINT, 1) $=$ T4

5 CONIINUE

330 IF (NQOVER) $425,425,335$ TO DO $420 \mathrm{~K}=1, \mathrm{NQOVER}$

$\mathrm{KCO}=\mathrm{NCOVER}+\mathrm{K}$

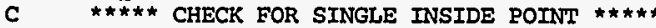

$\operatorname{SEG}(1)=\mathrm{CQ}(\mathrm{KCQ}, 1)$

340 SEG (1) $=1$ (1) $0-10,340,340$

IF (SEG $(1)-1,0) 350,350,345$

C $\star \star \star \star \star$ INSIDE POINT FOUND, ONLY ONE INTERSECTION POSSTBLE $\star \star \star \star \star *$

345 T12=1

$350 \mathrm{DO} 410 \mathrm{~J}=1,4$

$\mathrm{T} 1=\mathrm{QL}(\mathrm{J}, \mathrm{K}, 1)$
$\mathrm{T} 2=\mathrm{QL}(\mathrm{J}, \mathrm{K}, 2)$

OF INTERSECTION $\star \star \star \star \star ~$

$\mathrm{T} 4=(\mathrm{T} 1 * \mathrm{YN}(1)-\mathrm{T} 2 \star \mathrm{YO}(1)) / \mathrm{T} 3$

$\mathrm{T} 5=(\mathrm{T} 1 \star \mathrm{YN}(2)-\mathrm{T} 2 \star \mathrm{Y}$
$\mathrm{J} 1=2 *(\mathrm{MOD}(\mathrm{J}, 4)+1)$

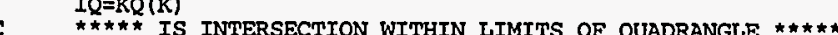
$T 6=\left(T 4-Q U A D\left(2 * J-1, I_{Q}\right)\right) *(Q U A D(J 1-1, I Q)-T 4)+(T 5-Q U A D(2 * J, I Q))$ * (QUAD (J1, IO) -T5)

1 IF (ABS (T6) $-1 . \mathrm{E}-4) 370,370,365$

365 IF T** CALCULATE FRACTION PARAMETER AND STORE IT $* * * * *$

380,395

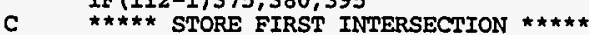

GO TO 390

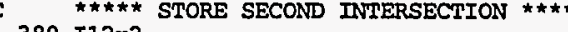

$\mathrm{I12}=2$
$\mathrm{IF}(\mathrm{T} 1-\mathrm{SEG}(1)) 385,405,405$

385 SEG $(2)=\operatorname{SEG}(1$

GO TO 410

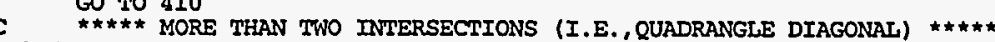
395 IF (T1-SEG (1)) $390,410,400$ 


\section{SEG $(2)=\mathrm{T}$}

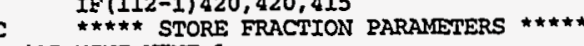

NINT $=$ NINT +1

$\operatorname{SEGM}(\mathrm{NINT}, 1)=\operatorname{SEG}(1)$

420 CONTINUE

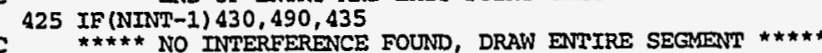

C $\star * \star \star \star \star$ NO INTEREERE

430 CALL $\operatorname{SCRIBE}(\mathrm{YN}, 2)$

C $435 \mathrm{M}=\mathrm{NINT}$
$440 \mathrm{M}=\mathrm{M} / 2$

$445 \mathrm{IF}=\mathrm{NINT}-\mathrm{M}$

$J=1$
$I=J$

$455 \mathrm{IM}=\mathrm{I}+\mathrm{M}$

$\operatorname{IF}(\operatorname{SEGM}(I, 1)) 460,470,470$

460 IF (SEGM (IM, 1)) $465,465,485$

465 IF (SEGM (I, 2)-SEGM(IM, 2)) 485, 485, 475

475 DO $480 \mathrm{~L}=1,2$

$$
\operatorname{SEGM}(I, L)=\operatorname{SEGM}(I M, L)
$$

IF (I) $485,485,455$

$85 \mathrm{~J}=\mathrm{J}+1$

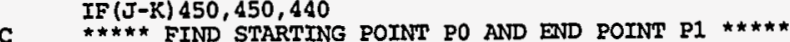

$490 \mathrm{P} 0=0.0$

IF (K-NINT) $500,500,515$

$500 \mathrm{P} 1=\operatorname{SEGM}(\mathrm{K}, 1)$

IF(P1) $510,505,505$

510 P0=

IF $(\mathrm{PO}-1,0) 495,530,525$

$515 \mathrm{P} 1=1.0$

政 IF (P0) 535, 535, 530

$525 \mathrm{PO}=1.0$

$\mathrm{Z}(1)=\mathrm{YO}(1) *(1 .-\mathrm{PO})+\mathrm{YN}(1) * \mathrm{PO}$

$z(2)=\mathrm{YO}(2) *(1,-\mathrm{PO})+\mathrm{YN}(2) * \mathrm{~B}$

CRIBE ( $Z, N P N)$

IF (PO-1.0) $535,540,540$

$\begin{aligned} 535 \mathrm{Z}(1) & =\mathrm{YO}(1) *(1 .-\mathrm{P} 1)+\mathrm{YN}(1) * \mathrm{P} 1 \\ \mathrm{Z}(2) & =\mathrm{YO}(2) *(1 .-\mathrm{P} 1)+\mathrm{YN}(2) * \mathrm{P} 1\end{aligned}$

540 RETURN

END

character* $(*)$ function maksym $(k, g p)$ $c * * *$ returns character string representation of symmetry operator dimension $\mathrm{gp}(3,4,192)$

character 1 xyz (3)

chact (23)

data fract $/ 1 / 24^{\prime}, 1 / 122^{\prime}, 1 / 8^{\prime}, 1 / 6^{\prime}, 5 / 24^{\prime}, 1^{\prime} 1 / 4^{\prime}, 7 / 24^{\prime}, 1 / 3^{\prime}$,

$3 / 8^{\prime}, 5 / 12,11 / 24^{\prime}, 1 / 2,13 / 24{ }^{\prime}, 7 / 121^{\prime}, 5 / 8^{\prime}, 2 / 3{ }^{\prime}$

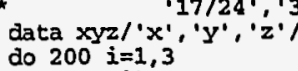

$200 i=1,3$.

iff $=0$

do $300 j=1,3$

fifix (gp $(i, j, k))$,ne. 0$)$ then

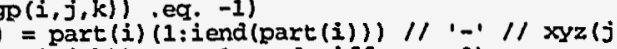

if part (if) $=$ part $(i)(1: i$ iend (part (i)f .eq. 0) $1 /$ xyz $(j)$

part (i) $=\operatorname{part}(i)(1: 2$

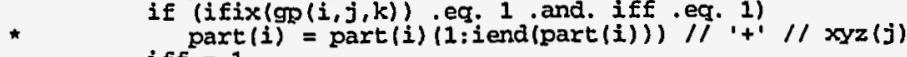
iff $=$

continue

grinue $9 p(i, 4, k)$

opval. 1t. -.01) then

(gaval it.0.) then . At. -01) then

$\operatorname{part}(i)=\operatorname{part}(i)(1:$ iend $(\operatorname{part}(i))) / /{ }^{-1}$

$\operatorname{part}(i)=\operatorname{part}(i)(1:$ iend $(\operatorname{part}(i))) / /++$

end if

gpval=abs (gpval)

do $301 \mathrm{~mm}=1$.

tf=float $(\mathrm{mm})$ *tfour

301

if (gpval.gt. (tf-.01) and. gpval.1t. (tf+.01)) iw=rm

end

$\operatorname{part}(i)=\operatorname{part}(i)(1: i e n d(\operatorname{part}(i))) / /$ fract(iw)

200 continue

= part(1) // part(2) // part(3)

return

SUBROUTINE MM $(X, Y, Z)$

C MUTIPY TWO MATRICE

DIMENSION $X(3,3), Y(3,3), z(3,3)$

$\mathrm{XIN} 1=\mathrm{X}(1,1)$

$\mathrm{x} 13=\mathrm{x}(1,3)$

$\mathrm{x} 21=\mathrm{x}(2,1)$

$\mathrm{X} 22=\mathrm{x}(2,2)$

$\mathrm{X} 23=\mathrm{x}(2,3)$

, $31=x(3,1)$

$x 33=x(3,3)$

$\mathrm{Y} 11=\mathrm{Y}(1,1)$

$\mathrm{Y} 12=\mathrm{Y}(1,2)$

$\mathrm{Y} 13=\mathrm{Y}(1,3)$

$Y 21=Y(2,1)$

$\mathrm{Y} 22=\mathrm{Y}(2,2)$ 
$Y 31=Y(3,1)$

$\mathrm{Y} 32=\mathrm{Y}(3,2)$

$Z(1,1)=X 11 * Y 11+X 12 * Y 21+X 13 * Y 31$

$2(3,1)=\times 31 * Y 11+\times 32 * Y 21+x 23 * \times 31$

$2(3,1)=x 31 * Y 11+x 32 * Y 21+x 33 \times Y 31$

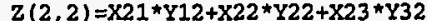

$Z(3,2)=X 31 * Y 12+X 32 * Y 22+X 33 \star Y 32$

$\mathrm{Z}(1,3)=\mathrm{X} 11 * \mathrm{X} 13+\mathrm{X} 12 * \mathrm{Y} 23+\mathrm{X} 13 * \mathrm{Y} 33$

$\mathrm{Z}(2,3)=\mathrm{X} 21 * \mathrm{Y} 13+\mathrm{X} 22 * \mathrm{Y} 23+\mathrm{X} 23 * \mathrm{Y} 33$
$\mathrm{Z}(3,3)=\mathrm{X} 31 * \mathrm{Y} 13+\mathrm{X} 32 * \mathrm{Y} 23+\mathrm{X} 33 * \mathrm{Y} 33$

RETURN

SUBROUTINE MV $(X, Y, z)$

C MATRIX * VECTOR

$Z(3)=X(3,3) * Y(3), Y(3), Z(3)$

$\mathrm{Y} 1=Y(1)$

\section{$Y 2=Y(2)$
$Y 3=Y(3)$}

$\mathrm{Z}(1)=\mathrm{X}(1,1) \star \mathrm{Y} 1+\mathrm{X}(1,2) * \mathrm{Y} 2+\mathrm{X}(1,3) * \mathrm{Y} 3$
$\mathrm{Z}(2)=\mathrm{X}(2,1) \star \mathrm{Y} 1+\mathrm{X}(2,2) \star \mathrm{Y} 2+\mathrm{X}(2,3) \star \mathrm{Y} 3$

$\mathrm{Z}(3)=\mathrm{X}(3,1) * \mathrm{Y} 1+\mathrm{X}(3,2) * \mathrm{Y} 2+\mathrm{X}(3,3) * \mathrm{Y} 3$

RETURN

SUBROUTINE NORM $(X, Y, Z$, ITYPE)

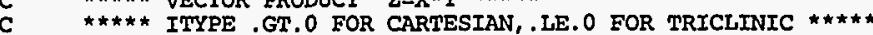
DIMENSION $X(3), Y(3), Z(3), Z 1(3)$

REAL $* 8$ AIN, ATOMID

CHARACTER * TITLE, TITLE2

COMMON NG, $A(9), A A(3,3), \operatorname{AAREV}(3,3), \operatorname{AANRK}(3,3), \operatorname{AID}(3,3)$

1 , AIN (140), ATOMID $(500)$, ATOMS $(3,500), \operatorname{BB}(3,3), \operatorname{BRDR}, \operatorname{CD}(8,20)$

2

$4, \operatorname{ORGN}(3), \operatorname{PAC}(3,5), \operatorname{PAT}(3,3), Q(3,3), \operatorname{REFV}(3,3), \operatorname{RES}(4), \operatorname{RMS}(5), \operatorname{SCALI}$

5 , SCAL2, SCL, SYMB (3,3), TAPER, THETA, TITLE (18), TITLE2 (18), TS $(3,96)$

6 , VIEN, VT $(3,4), \operatorname{V1}(4)$

DO $125 I=1,3$

$I 1=M O D(I+3,3)+1$

$\mathrm{T} 1=\mathrm{X}(\mathrm{I} 1) * \mathrm{Y}(\mathrm{I} 2)-\mathrm{X}(\mathrm{I} 2) * \mathrm{Y}(\mathrm{II})$

05 IF (ITYPE) $115,115,105$

$z(T)=T 1$

$115 \mathrm{Z1}(\mathrm{I})=\mathrm{T} 1$

$115 \mathrm{Z1}(\mathrm{I})=\mathrm{T} 1$

IF (ITYPE) $135,135,300$

135 CALI MV (BB, 21, 2$)$

300 RETURN

END

SUBROUTINE NUMBUR (W, W2 , HGT, DIST, THT, ND)

CONVERT BOND DISTANCE FOR PLOTTING IN ORTEP

CHARACTER *1 ITEX ITX

EQUIVALENCE (ITEX (1) ITTX)

C----COMPUTE NUMBER OF CHARACTERS FOR OUTPUT $\mathrm{NC}=\mathrm{ND}+1$
$\mathrm{XD}=\mathrm{DIST}$

IF (XD.LT.1.0) GO TO 20

IF (NC.GE. 9 ) 60 TO 30

$\mathrm{XD}=\mathrm{XD} / 10.0$

GO TO 10.0

TORMAT STATEMENT

WRITE (IFMT, 25) NC, ND

25 FORMAT (' (F', II, ' ' ' 'II, ')' ')

WRITE (ITXT, IFMT) DIST

CALL SIMBOL (W, W2, HGT, ITEX, THT, NC)

30 RETURN

SUBROUTTNE PAXES (DCODE, ITTPE)

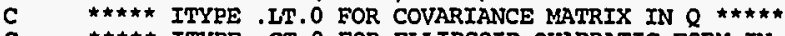

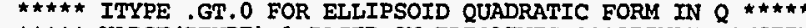

***** XABSF (ITYPE) $=1$ BASED ON TRICLINIC COORDINATE SYSTEM $* \star \star * *$

* $* * * *=2$ OR 3 FOR WORRING OR REFERENCE CARTESIAN SYSTEMS $* * * * *$

DIMENSION $X(3)$

REAL*8 DCODE, D100, D100K

REAL $* 8$ AIN, ATOMID

CHARACTER* 4 TITLE,

CHARACTER $\star 8$ CHEM

COMMON NG, $A(9), \operatorname{AA}(3,3), \operatorname{AAREV}(3,3), \operatorname{AANRK}(3,3), \operatorname{AID}(3,3)$

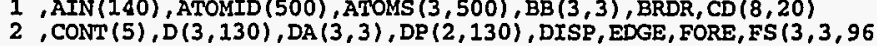

3 , IN, ITILT, KD $(5,20)$, LATM, NATOM, NCD, NJ, NJ2, NOUT, NSR, NSYM

$4, \operatorname{ORGN}(3), \operatorname{PAC}(3,5), \operatorname{PAT}(3,3), 0(3,3), \operatorname{REFV}(3,3), \operatorname{RES}(4), \operatorname{RMS}(5), \operatorname{SCAL} 1$

5 , SCAL2, SCL, SYMB (3,3), TAPER, THETA, TITLE (18), TITLE2 (18), TS $(3,96)$

, VIEN, $(3,4), V 1(4), V 2(3$

COMMON / PARMS/ CHEM (505), EV $(3,505), \mathrm{P}(3,505), \mathrm{BA}(3,3,505)$

1 , IDENT $(2,505)$, MAXATM

$D 100=100$.

$\mathrm{D} 100 \mathrm{~K}=100000$.

$I T=I A B S(I T Y P E)-1$

TF (NSYM-KS) 105,115,115

$105 \mathrm{NG}=4$

$115 I I=D C O D E / D 100 K$

IF (NATOM-II) 125, 130, 13

$\mathrm{NG=5}$

130 IF (II) $125,125,135$

C 130 IF(II) 125,125,135

135 CALL TMAS (PA $(1,1$, II), FS $(1,1$, KS $)$, PAT)

IF (IT-1) 160,145,155

C 145 CALT TRMANSFORM TO CARTESIAN SYSTEMS $* * * \star * *$

GO TO 175

175 (Dam, Aarev,

GO TO 175

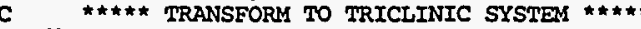

62 Do $165 J=1,9$
$165 \operatorname{PAC}(J, 1)=\operatorname{PAT}(J, 1)$ 
Go TO 175

170 CALI MM(AA, PAT, PAC)

175 DO $205 \mathrm{~J}=1,3$

IF (ITYPE) 195, 195, 185

$185 \times(\mathrm{J})=1 . /(\mathrm{T} 1 * \mathrm{~T} 1)$

GO TO 205

$195 X(\mathrm{~J})=\mathrm{T} 1 * \mathrm{~T} 1$

205 RMS(J) $=$ TI

DO $245 \mathrm{I}=1,3$

$\mathrm{T} 1=0.0$

225 T1=T1 $225 \mathrm{PAC}(\mathrm{I}, \mathrm{K}) * \mathrm{PAC}(\mathrm{J}, \mathrm{K}) * \mathrm{X}(\mathrm{K})$

$Q(J, I)=T I$

300 RETURN

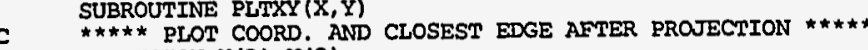
DIMENSION $X(3), Y(2)$

REAL*8 AIN, ATOMID

COMMON NG A (9), AA (3,3) AAREV $(3,3)$ AAWRK $(3,3)$, AID $(3,3)$

CATN (140), ATOMTD (500), ATONS $(3,500), \operatorname{BB}(3,3), \operatorname{BRDR}, \mathrm{CD}(8,20)$

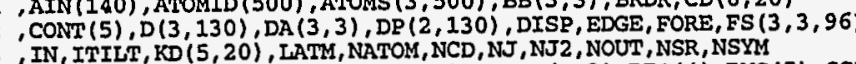

$, \operatorname{ORGN}(3), \operatorname{PAC}(3,5), \operatorname{PAT}(3,3), Q(3,3), \mathrm{REN}(3,3), \mathrm{RES}(4), \operatorname{RMS}(5), \operatorname{SCAL} 1$

, SCAL2, SCL, SYMB (3,3), TAPER, $(3), V A(3), V 5(3), V 6(3), W R V(3,3)$

XING $(3), X 0(3), X T(3)$

Th=1.

IF (VIEW) $125,125,110$

$110 \mathrm{~T} 4=\mathrm{VIEW}-\mathrm{X}(3)$

$115 Y(1)=-99$.

$Y(2)=-99$.

$\mathrm{T} \mathrm{T}=\mathrm{VIEN} / \mathrm{T}$

$125 \begin{aligned} \mathrm{Y}(1) & =\mathrm{X}(1) \star \mathrm{T} 1+\mathrm{XO}(1) \\ \mathrm{Y}(2) & =\mathrm{X}(2) * \mathrm{~T} 1+\mathrm{XO}(2)\end{aligned}$

$Y 1=X I N G(1)-A B S(Y(1) * 2 .-X I N G(1)$

$T 1=X I N G(1)-A B S(Y(1) * 2 .-X L N G(1))$

EDGE $=$ AMTN1 $(T 1, T 2) \star 5$

IF 1 TA $-V I E$
EDGE $=-99$.

300 RETURN

SUBROUTINE PLOT ( $x, y$, ipen)

SUBROUTINE PLOT(x,y,ipen)

if (ndraw eq. 1) cail pensc ( $x, y$, ipen

if (ndraw .eq. 2) call penps $(x, y$, ipen

if (ndraw .eq. 3) call penhp $x, y, 1$ pen

if (ndraw .eq. 9) call pensc $(x, y$, ipen

return

SUBROUTINE PRELIM

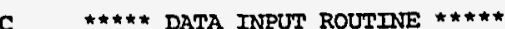

BFAL*8 8 TD

IMENSION $B(9)$

CAL*B AIN, ATOMIO

GHARACTER *4 TITLE, TITLE2

CCOMACTER $\star 8$ CHEM $(9), \operatorname{AAA}(3,3), \operatorname{AAREV}(3,3), \operatorname{AANRK}(3,3), \operatorname{AID}(3,3)$

1 , AIN (140), ATOMID (500), ATONS (3,500), BB (3,3), BRDR, CD $(8,20)$

2 '

$4, \operatorname{ORGN}(3), \operatorname{PAC}(3,5), \operatorname{PAT}(3,3), Q(3,3), \operatorname{REEV}(3,3), \operatorname{RES}(4), \operatorname{RMS}(5), \operatorname{SCAL} 1$

(18), TS $(3,96)$

$6, V I E N, V T(3,4), V 1(4), V 2(3), V 3(3), V 4(3), V 5(3), V 6(3), \operatorname{WRKV}(3,3)$

7 , XLNG (3), XO(3), XT(3)

COMMON /PARMS/ CHEM(505), EV (3, 505), P(3,505), PA $(3,3,505)$

C

CHARACTER*73 INQ, QUE, hque

character*80 card

character $\$ 24$ sympart (3)

dharsion $\star 36$ maks

c MENSIONS $* \star \star \star * *$

106 FORMAT (i1, f8.6,5F9.6)

READ (IN, 107) card

107 format (a)

(NED, 107) card

WEAD (card, 106) iflag, (A(I), I=1,6)

$T 1=\operatorname{ABS}(\mathrm{A}(4))-1$

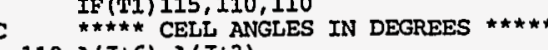

$A(J+6)=A(J+3)$

(J+6)*1.745329E-2)

$115 * \pi * *$ COSINES OF CELL ANGLES $* * \pi * *$

* $*$ *

$120 \operatorname{AID}(J, J)=1$.

$\operatorname{AID}(J+1,1)=0$.

$* \star * * *$ STORE METRIC TENSOR $* * \star \star *$
$125 \mathrm{AA}(\mathrm{J}, \mathrm{J})=\mathrm{A}(\mathrm{J}) \star \star 2$

$A A(1,2)=A(1) * A(2) * A(6)$

$\mathrm{AA}(1,3)=\mathrm{A}(1) * \mathrm{~A}(3) * \mathrm{~A}(5)$
$\mathrm{AA}(2,3)=\mathrm{A}(2) * \mathrm{~A}(3) * \mathrm{~A}(4)$

$A A(2,1)=A A(1,2)$

AA $(3,1)=A A(1,3)$

* $* \star *$ INVERT KETRIC TENSOR $* * * *$

CALL AXEQB (AA, BB, AID , 3)

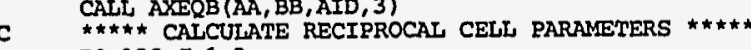

DO $128 J=1,3$

$8 \mathrm{~B}(\mathrm{~J})=\operatorname{SRRT}(\mathrm{BB}(\mathrm{J}, J)$

$B(6)=B B(1,2) /(B(1) \star B(2)$

$B(5)=B B(1,3) /(B(1) * B(3)$
$B(4)=B B(2,3) /(B(2) * B(3)$

DO $130 \mathrm{~J}=1,3$

$130 \quad B(J+6)=\operatorname{ARCCOS}(B(J+3))$ 


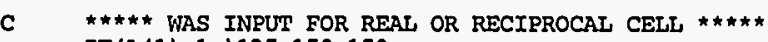
IF (A (1) -1.)135, 150,150

135 DO $140 \mathrm{~J}=1$,

$T 1=A A(J, 1)$
$A A(J, 1)=B B(J, 1)$

$\mathrm{BB}(\mathrm{J}, 1)=\mathrm{T}$

$T 1=A(J)$

$140 \begin{aligned} & A(J)=B(J) \\ & B(J)=T 1\end{aligned}$

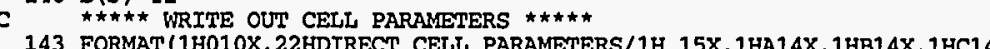
$1 \times$ 5HATPHA $10 x$, FORMAT ( $1 \mathrm{H}, 10 \mathrm{X}$, F9 .5, 2F15.6, 3F15.3/1H , 48X, 6HCOSINE, F12.8, 2F15.8) 147 FORMAT(1H010X, 26HRECIPROCAL CELI PARAMETERS/1H 15X, 2HA $13 \mathrm{X}, 2 \mathrm{HB} \star 13 \mathrm{X}$ $1,2 \mathrm{HC}^{\star} 13 \mathrm{X}, 6 \mathrm{HALPHA} * 9 \mathrm{X}, 5 \mathrm{HBETA} * 10 \mathrm{X}, 6 \mathrm{HGAMMA}$ *

150 IF (NOUT.GE.0)

\&WRITE (NOUT, 143)

(A)

IF (NOUT,GE.0)

\&WRITE (NOUT, 147)

IF (NOUT.GE. 0 )

(NOUT, 145) (B(I), I=1,3), (B(I), I=7,9), (B(I), I=4, 6)

CALT AXES (AID, AID $(1,2)$, REFV, 0 )

CAIL $M M(A A, P E V V, A R P E V)$

DO $160 \quad I=1,3$

DO $160 \mathrm{~J}=1,3$

AAWRK $(J, I)=$ AARE

$160,(J, I)=R E F(I, J)$

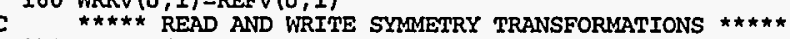

171 FORMAT ( $1 \mathrm{HO}, 10 \mathrm{X}, 24 \mathrm{HSXMMETRY} \mathrm{TRANSFORMATIONS/1H} 14 \mathrm{X}, 3 \mathrm{HNO})$

c 171 FORMAT (1HO10X, 24HSYMMETRY TRANSFORMATIONS/1H 14X, 3ENO.12X, 13HTRANS

1FORMED X18X,13HTRANSFORMED Y18X, 13HTRANSFORMED $\mathrm{Z}$ )

175 FORMAT $(1 \mathrm{H}, 13 \mathrm{X}, \mathrm{I2}, 3(\mathrm{~F} 13.6, \mathrm{~F} 4.0,2 \mathrm{H}$ X, F4.0, $2 \mathrm{H} \mathrm{Y}, \mathrm{F} 4.0,2 \mathrm{H} \mathrm{Z})$

176 FORMAT $(1 \mathrm{H}, 13 \mathrm{X}, \mathrm{I2}, 5 \mathrm{X}, \mathrm{a})$

IF (NOUT.GE.0)

DO $190 \quad I=1,96$

READ (IN, 107) card

if (iflag. eq. 0) READ (card, 173) IS, (TS (J, I) , (FS (K, J, I), K=1, 3) , J=1, 3)

if (iflag.eq. 1)

read (card. 1771) is

1771 format(i1)

ipart $=1$

do $1772 j \mathrm{k}=1,3$

1772

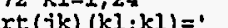

1773

if (card(jk:jk).eq. ' ") go to 1776

do $1774 \mathrm{kl}=\mathrm{jk}, 80$

if (card (kl:kl).eq.' ' or. card $(k l: k l)$.eq. ', ') then go to 1775

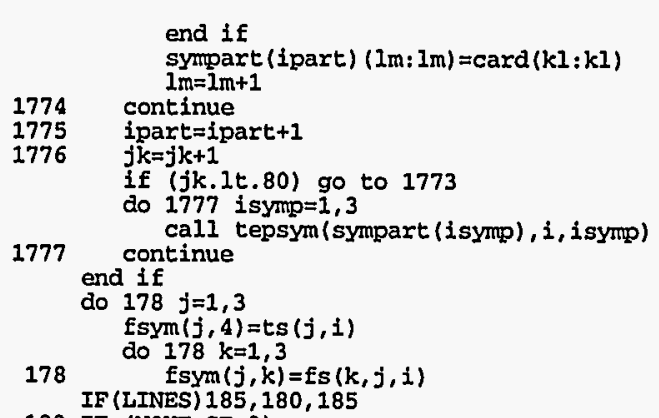

180 IF (LINES) $185,180,185$

(NTRTITE $(J), J=1,18)$

IF (NOUT.GE.0)

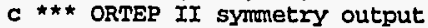

c 185 IF (NOUT. GE. 0)

\&WRTE (NOUT, 175) I, (TS (J, I), (ES (K,J,I), K=1,3), J=1, 3)

185 if (nout.ge. 0) WRITE (NOUT, 176) I, maksym (1, fsym)

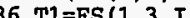

$\mathrm{T} 1=\mathrm{AMOD}(\mathrm{T} 1 * \mathrm{FS}(2,3,1), 1.) \star 6.28318531$

$\mathrm{T} 2=\cos (\mathrm{T} 1)$

$\mathrm{T} 1=\operatorname{SIN}(\mathrm{T} 1)$

$187 \operatorname{VT}(J, 1)=A I D(J, 1)$

$\operatorname{VT}(1,1)=T 2$

$\operatorname{VT}(2,1)=-\mathrm{T} 1$

$\operatorname{VT}(1,2)=T I$

CALL MM (VT, $Q, P A C)$

caris

$\mathrm{NG}=1$

$$
\text { CALL ERPNT }(0 . D 0,0)
$$

195 N=97

$I=96$
$N S Y M=I$
$I S W=I S$

NATOM=0

207 FORMAT (11HO NO. ATOM 8X, 1HXX10X, 1HY10X, 1HZ13X, 3HB118X, 3HB228X, $3 \mathrm{HB} 33$ 8X, 3HB128X, 3HB138X, 10HB23 TYPE)

FORMAT (1H, I3, 1X, A6, 3F11,6,5X,6F11,6, F5,0)

210 FORMAT(1H , I3, 1X,A6, 3F11.6, 5X, 2F11.6, 4F11.0,F5.0)

211 FORMAT (A6, 3X, 6F9.0) 213 FORMAT (I1, F8.0,5F9.0,7X,F2.0

$$
\text { LINES=LINES+2 } 20,215,215
$$

215 LINES $=-1$

220 IF (NOUT.GE. 0)

if (isw.eq. 2) the 


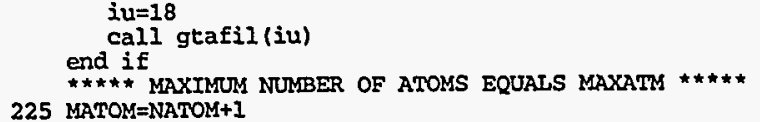

DO 245 I=MATOM, MAXATM

IF (ISW. EO I) GO TO 226

CALE CALU SPECIAL PURPOSE READIN ROUTINE $\star * \star * *$

CALL readin (Iu, CHEM(I), IDENT(1,I),IDENT $(2, I), P(1, I), P(2, I), P(3, I)$

$1, I T 1, I S, \operatorname{PA}(1,1, I), \operatorname{PA}(2,1, I), \operatorname{PA}(3,1, I), \operatorname{PA}(1,2, I), \operatorname{PA}(2,2, I)$

$2, \operatorname{PA}(3,2, I), \operatorname{PA}(1,3, I))$

$K=I T 1+1$

$\mathrm{K}=1 \mathrm{~T} 1+1$

226 continue

READ (IN, 107) card

政

$\operatorname{DENT}(1, I)=\mathrm{V} 1(1)$
$\operatorname{DENT}(2, I)=\mathrm{V} 1(2)$

$\mathrm{K}=1,+\mathrm{T} 1$

$227 \mathrm{~K}=1$

(FLOAT $(K-1)-T 1) 227,228,227$

228 continue

READ (IN, 107) card

RED (card 213 ) IS (PA (J, $1, J=1,7)$

229 IF(LINES) $230,230,232$

230 IF (NOUT.GE.0)

QWRITE (NOUT, 177) (TITLE (J) ,J=1, 18)

IF (NOUT.GE.0)

$232 \operatorname{IF}(P A(3,1, I)-10000) 235,234,$.

作

GO TO 238

235 IF (NOUT.GE. 0 )

\&WRITE (NOUT, 209) I, CHEM(I), ( $(J, I), J=1,3),(\operatorname{PA}(J, 1, I), J=1,7)$

238 GO TO $(244,239,241,242,244)$

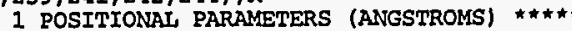

$40 P(J, I)=P(J, I) / A(J)$

GO TO 244

* $* \star \star * \star$ TYPE 2 POSITIONAI PARAMETERS, STANDARD CARTESIAN $* * \star *$

$241 \mathrm{~V} 1(1)=\mathrm{P}(1, I)$

GO TO 243

C $* * * \star *$ TYPE 3 POSITIONAL PARAMETERS $* * * * *$ T

***** CYIINDRICAL COORDINATES REFERRED TO STANDARD CARTESIAN $* * * * *$

$242 \mathrm{~T} 2=P(2, I) * 01745329252$

$\mathrm{V} 1(1)=\mathrm{V} 1(1)+\mathrm{P}(1, I) * \operatorname{CoS}(\mathrm{T} 2)$
$\mathrm{V} 1(2)=\mathrm{V} 1(2)+\mathrm{P}(1, I) * \operatorname{SIN}(\mathrm{T} 2)$

CALL VM(VI,Q,P(1,I)

244 IF (IS) 24

if (isw.eq. 2) close(iu)

CALL ERPNT $(0 . D 0,0)$

I=MAXATM

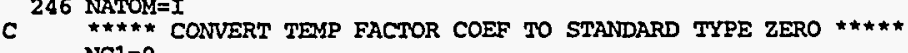

$450 I=1$, NATOM

C interim fix for IBM AIX

$\mathrm{K} 9=$ ?

$\mathrm{K}=1 .+\mathrm{PA}(\mathrm{Kg}, 1, \mathrm{I})$

$250 \mathrm{~T} 1=.1$

GO TO 405

GO TO $(270,260,265,265,270,260,400,405,270,260,270,450), \mathrm{K}$

260 DO $262 \mathrm{~J}=4,6$

$262 \operatorname{PA}(J, 1, I)=\operatorname{PA}(J, 1, I) * .5$

GO TO 270

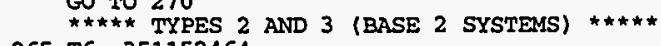

IF $(K-4) 270,260,270$

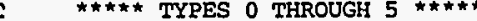

$270 \operatorname{IF}(\operatorname{PA}(2,1, I)) 400,400,272$

$\begin{aligned} 272 & \text { DO } 300 \quad \mathrm{~J}=1,3 \\ \text { DO } 300 \quad \mathrm{~L}=\mathrm{J}, 3 & \end{aligned}$

$D 2=\pi 6$

IF ( $\mathrm{K}-5) 285,275,275$

275 IF $(K-6) 280,280,281$

280 T2 $2=B(\mathrm{~J}) * \mathrm{~B}(\mathrm{I}) * \mathrm{~T} 2 * .25$

GO TO 285
C TY* 8 AND 9 (U(I,J) TENSOR SYSTEMS) $\star * \star \star \star *$

$281 \mathrm{~T} 2=\mathrm{B}(\mathrm{J}) * \mathrm{~B}(\mathrm{~L})$

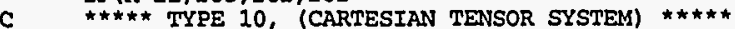

$285 \mathrm{IF}(\mathrm{J}-\mathrm{L}) 290,287,290$

$287 \operatorname{VT}(J, J)=T 2 * \mathrm{PA}(\mathrm{J}, 1, I)$

$290 \mathrm{M}=\mathrm{J}+\mathrm{L}+1$

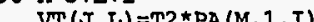

$\operatorname{VT}(J, L)=T 2 * \operatorname{PA}(M, 1$
$\operatorname{VT}(L, J)=V T(J, L)$

300 CONTINUE

C $\star$.

IF (K-11) 310,305,305

305 CAUL MM(VT,Q, PAC)

310 CALL MA (VT, AA, DA)

C CALL EIGEN (DA, RMS, PAT)

IF(RMS (1)) $325,325,320$

$320 \operatorname{IF}$ (NG) $350,360,330$

330 NG1 $=1$

CALL ERPNT (DBLE (I) *D100K+55501.D0,0)

C

40 T3=SIGN (SRRT (ABS (RMS (1) +RMS (2) +RMS (3)) /3.), RMS (1))

$345 \mathrm{~J}=1,3$
$342 \mathrm{~K}=1,3$

$342 \operatorname{PA}(J, K, I)=\operatorname{REFV}(J, K)$

345 EV $(J, I)=T 3$
GO TO 450 
350 IF (NG+6) 340,340,352

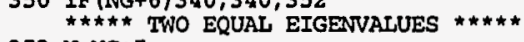

$52 N=N G+5$

CALI UNITY (PAT (1,N),V1, -1)

IF (ABS (VMV

354 CONTINUE

356 CALL MM(AA, DA, VTr)

$\operatorname{CALL} \operatorname{AXES}(\mathrm{V} 1, \mathrm{REFV}(1, \mathrm{~K}), \mathrm{DA},-1)$

DO $359 \mathrm{~K}=1,3$

$4=M O D(N+K-2,3)+1$

$358 \mathrm{PA}(\mathrm{J}, \mathrm{L}, \mathrm{I})=\mathrm{DA}(\mathrm{J}, \mathrm{K})$

$359 \operatorname{EV}(\mathrm{L}, \mathrm{X})=\operatorname{SIGN}(\mathrm{SQRT}$ (ABS (VMV (DA $(1, \mathrm{~K}), \mathrm{VT}, \mathrm{DA}(1, \mathrm{~K}))$ )), RMS (L))

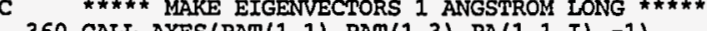

$360 \mathrm{CALL}$ AXES $(\operatorname{PAT}(1,1), \operatorname{PAT}(1,3), \operatorname{PA}(1,1,1),-1)$

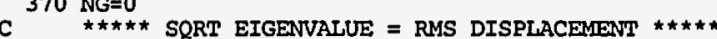
DO $375 \mathrm{~J}=1,3$

$75 \mathrm{EV}(\mathrm{J}, \mathrm{I})=\operatorname{SIGN}(\operatorname{SQRT}(\mathrm{ABS}(\mathrm{T} 2)), \mathrm{T} 2)$$$
\text { GO TO } 450
$$

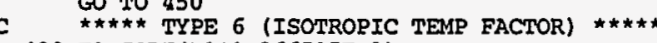

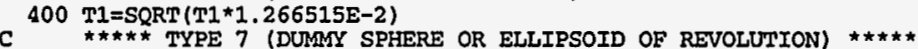

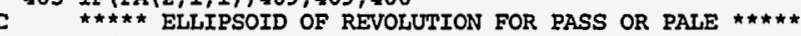

$406 \mathrm{EV}(1, I)=\mathrm{T}$

$$
\begin{aligned}
& \operatorname{EV}(2, I)=\mathrm{PA}(2,1, I) \\
& \operatorname{EV}(3, I)=\mathrm{PA}(2,1, I)
\end{aligned}
$$

GO TO 411

C $\star \star \star \star \star$ SPHERE FOR PEAK OR PIT, OR A GENERAL SPHERE ATOM $\star \star \star \star \star ~$ $409 \mathrm{DO} \quad 410 \mathrm{~J}=1,3$

411 IF (PA (3,1,I) $430,430,415$

C

$415 \mathrm{DO} 417 \quad \mathrm{~J}=1,2$

TD $=\operatorname{PA}(J+2,1 ; I)$
CALL ATOM $(T D, V T(1, J)$

IF (NG) $416,417,416$

416 CALI ERPNT (TD, 0$)$

60 TO 430

417 CONTINU

$$
\text { CALL DIFVIVT }(1,2), \operatorname{VT}(1,1), \mathrm{V} 1
$$$$
\text { T11 }=\operatorname{SQRT}(\mathrm{VMV}(\mathrm{V} 1, \mathrm{AA}, \mathrm{V} 1)
$$

Do $418 \mathrm{~J}=1,3$

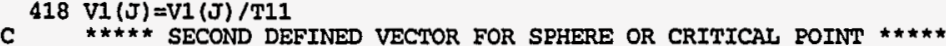

$$
\begin{aligned}
& D O \quad 20 \quad \mathrm{~J}=3,4 \\
& \text { IF(TD.EQ.0.0) GO TO } 422 \\
& \text { CALL ATOM(TD, VT }(1, J))
\end{aligned}
$$

419 CALL ERPN

420 CONTINUS

CALL DIFV (VT $(1,4), \mathrm{VT}(1,3), \mathrm{V} 2)$

$=\operatorname{SQRT}(\mathrm{VMV}(\mathrm{V} 2, \mathrm{AA}, \mathrm{V} 2))$

$421 \mathrm{~V} 2(\mathrm{~J})=\mathrm{V} 2(\mathrm{~J}) / \mathrm{T} 11$

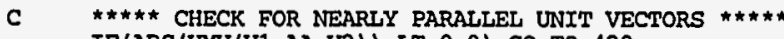
IF (ABS (VNV (V1, AA, V2)) ). LT. 0.9) G0 TO 429

$422 \mathrm{~T} 22=1.0$

DO $424 \mathrm{~J}=1,3$

T11=ABS (VMV (V1, AA, $\operatorname{REFV}(1, J)))$

IF (T22. LE.T11) GO TO 424

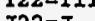

424 CONTINU

$425 \mathrm{~J}=1,3$

425 V2 (J)

CALL AXES (V1, V2, PA $(1,1, I),-1)$

GO TO 450

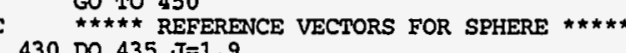

430 DO $435 J=1,9$
35 PA $(J, 1, I)=R E F V(J, 1)$

450 NG=0

NG=0

LINES=LINES+2

455 LINES $=-1$

458 IF (NOUT.GE.0)

EWRITE (NOUT, 461)

DD

IF (LTNES) $465,462,465$

461 FORMAT (10HONO. ATOM 8X, 1HX10X, 1HX10X, 1HZ13X, 7HRMSD 1 4X, 7HRMSD 2 $1 \mathrm{X}, 7 \mathrm{HRMSD} 3{ }^{\prime}$

62 IF (NOUT.GE.0)

(WWITE (NOU, 177) (TITLE $(J), J=1,18$ )

IF (NOUT.GE. 07 )

463 FORMAT (1H , I3, 1X, A6, 3F11.6)

\&WRITE (NOUT, 209) I, CHEM(I), ( $(J, I), J=1,3),(E V(J, I), J=1,3$

IF (NG1) 999, 999,470

99 RETUR

SUBROUTTNE PRIME

C $\star * \star \star$ GENERAL INITIALIZATION OF PRIME PARAMETERS $* \star * *$

REAL*8 AIN, ATOMID

CHARACTER *4 TITLE, TITLE2

COMMON NG, A (9), AA $(3,3), \operatorname{AAREV}(3,3), \operatorname{AAWRK}(3,3), \operatorname{AID}(3,3)$

CONT $(5), \operatorname{ATOMID}(500), \operatorname{ATOMS}(3,500), \mathrm{BB}(3,3), \operatorname{BRDR}, \mathrm{CD}(8,20)$

D

, IN, ITTLT, KD $(5,20)$, LATM, NATOM, NCD, NJ, NJ2, NOUT, NSR, NSYM

, ORGN (3), PAC $(3,5), \operatorname{PAT}(3,3), Q(3,3), \operatorname{REFV}(3,3), \operatorname{RES}(4), \operatorname{RMS}(5), \operatorname{SCAL}$

, SCAL2, SCL, SYMB $(3,3)$, TAPER, THETA, TITLE (18), TITLE2 $(18), \operatorname{TS}(3,96)$

, VIEN $(3)(3,4), V 1(4), V 2(3), V 3(3), V 4(3), V 5(3), V 6(3), \operatorname{VRKV}(3,3)$

,XENG(3),XO(3),XT(3)

COMMON / PARMS/ CHEM(505), EV $(3,505), P(3,505), \mathrm{PA}(3,3,505)$

1 , IDENT $(2,505)$, MAXATM

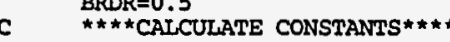


DO $2950 \quad I=1,5$

$2950 \operatorname{coNT}(I)=\operatorname{SORT}(1 . /(2 . \bullet(1 .+\cos (3.141593 \quad / 2 . * \star I))))$

c DISP $=.005$

Fisp $=0.86$

ITILT $=0$

MATM $=0$

$\mathrm{NCD}=0$

3000 ORGN(J) $=0,3$

$\operatorname{RES}(1)=.75$

$\operatorname{RES}(2)=.5^{\star}$ res $(1)$

SCALI $1=1.0$

SCAL2 $=1.54$

SCL $=1.54$

DO $3005 \quad I=1,3$

$\operatorname{SYMB}(I, I)=1$.

$3005 \operatorname{SYMB}(I+5,1)=0$.

TAPER $=.375$

THETA $=0.0$

$\mathrm{VIEW}=0.0$

XING $(1)=10.5$

$\mathrm{XING}(2)=8.0$

$\mathrm{XO}(1)=5.25$

$x O(3)=0.0$

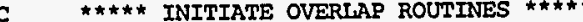

CALL LAP500 (0)

RETURN

END

C DIMENSION D $(3,129), \mathrm{DP}(2,129), \mathrm{X}(3), \mathrm{XO}(3)$

$T 3=V I E N-X(3)$

DO $145 I=I 1, I 2, I 3$

$T 1=D(1, I)+X(1)$

$T 2=D(2, I)+X(2)$

$120 \mathrm{~T} 4=\mathrm{VIEN} /(\mathrm{T} 3-\mathrm{D}(3, \mathrm{I})$

$\mathrm{T} 1=\mathrm{T} 1 * \mathrm{~T} 4$

$135 \mathrm{DP}(1, I)=\mathrm{T} 1+\mathrm{XO}(1)$

$145 \mathrm{DP}(2, I)=\mathrm{T} 2+\mathrm{XO}(2)$

RETURN

END

C SUBROUTINE RADIAL (ND)

FROM TWO CONJUGATE VECTORS $* * \hbar * \star$

***** ORTHONORMAI VECTORS PRODUCE 8-128 SPOKED CIRCLE

REAL $* 8$ AIN, ATOMID

CHARACTER 4 TITLE, TITLE2
COMMON NG, A $(9), A A(3,3)$, AAREV $(3,3), \operatorname{AAWRK}(3,3), \operatorname{AID}(3,3)$

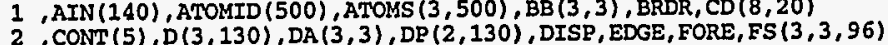

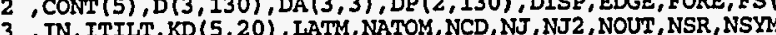

, ORGN(3), PAC $(3,5), \operatorname{PAT}(3,3), O(3,3), \operatorname{REEV}(3,3), \operatorname{RES}(4), \operatorname{RMS}(5), \mathrm{SCAL}^{-1}$

5 , SCAL2, SCL, SYMB $(3,3)$, TAPER, THETA, TITLE (18), TITLE2 (18), TS $(3,96)$
6 , VIEN, VT (3,4), VI (4), V2 (3),V3 (3), V4 (3), V5 (3), V6 (3), WRKV $(3,3)$ ,

T1 $=$ DA $(J, 1)$

$D(J, 1)=T 1$

$D(J, 129)=\mathrm{T} 1$

$\mathrm{D}(\mathrm{J}, 65)=-\mathrm{TI}$

$T I=D A(J, 2)$

$\mathrm{D}(\mathrm{J}, 33)=\mathrm{T} 1$

$115 \mathrm{D}(\mathrm{J}, 97)=-11$

Do $135 \mathrm{~K}=1, \mathrm{in}$

$\mathrm{KDEL}=2 \star \star(6-\mathrm{K})$

KDEL $1=$ KDEL +1

DO $135 \mathrm{~L}=\mathrm{KDEL} 1,65, \mathrm{KDE}$

$\mathrm{J}=\mathrm{L}-\mathrm{KDEL}$

$M=L-K D E L 2$

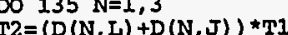

$D(N, M)=T 2$

$135 D(N, M+64)=-T 2$

RETURN

END

utine readin (iu, chem, id1, id2, x1, x2, x3, it, is, b1,b2,b3,b4 b5, b6, btype)

integer $\star 2$ id1, id2

character $* 1$ chain

character $* 3$ res

character $* 4$ atom

character $* 6$ rec

b1 $=.1$

$\mathrm{b} 2=0$

$b 4=0$

$\mathrm{b} 6=0$

btype $=7$

idi $=0$

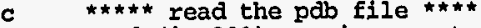

, chain, id2 $, x 1, \times 2, \times 3,0 c c, t f$

201 format $(a 6,15,1 x, a 4,1 x, a 3,1 x, a 1, i 4 ; 4 x, 3 f 8.0,2 f 6.0)$

if (atom. eq. ' $N$ ') $i d 1=1$

if atom. eq.' $C A$, $1 \mathrm{~d} 1=2$

if atom. eq. o if then id1 $=4$

b1 $=.15$

end if

atom $(2: 4) / /$ res

(iu, 202, end=203) rec

backspace (iu)

203 is $\begin{aligned} & \text { return } \\ & \text { istuma }\end{aligned}$

return 
end

SERIOUTINE recycle

CHARACTER 44 TITLE, TITLE

COMMON NG $, A(9), A A(3,3), \operatorname{AAREV}(3,3), \operatorname{AAWRK}(3,3), \operatorname{AID}(3,3)$

(140) ATOMD (500) ATOMS (3,500), BB (3,3), BRDR, CD $(8,20)$

$2, \operatorname{CONT}(5), D(3,130), D A(3,3), D P(2,130)$, DISP, EDGE, FORE, FS $(3,3,96)$

3 , IN, ITILT, KD (5, 20), LATM, NATOM, NCD, NJ, NJ2, NOUT, NSR, NSYM

$4, \operatorname{ORGN}(3), \operatorname{PAC}(3,5), \operatorname{PAT}(3,3), Q(3,3), \operatorname{REFV}(3,3), \operatorname{RES}(4), \operatorname{RMS}(5), \operatorname{SCAI}$

5 , SCAL2, SCL, SXMB $(3,3)$, TAPER, THETA, TITLE (18), TITLE2 $(18), T S(3,96)$

,VIEN, VT (3,4),V1 (4), V2 (3), V3 (3),V4 (3),V5 (3), V6 (3), $\operatorname{VRKV}(3,3)$

,XING (3), Xo(3), XI)

NEXT, NBACK, INQ, QUE (96) , hque(96)

, ndraw, norient, nvar

if (ndraw.eq.2 .or. ndraw. eq.3) call getpap

$c * * *$ find 201 (203) instruction

do 545 i=1, nque
if (que $(i)(7: 9)$.eq. '201' .or. que(i) $(7: 9)$.eq. '203') then next $=1$ go to 570 end if

545 Continue
2E* 570 IATM=0

DO $580 \quad I=1,500$

ATOMID $(I)=0$

$580 \operatorname{ATOMS}(\mathrm{J}, I)=0$

RENT

END
SUBROUTINE SCRIBE (Y, NPEN)
DIMENSION $Y(2)$, YO (2)

C DIMENSION $\star \star \star \star \star$ SUBROUTINE WHICH LINKS WITH THE PLOTTER-SPECIFIC SUBROUTINES

IF (NPEN-3) 210,205,205

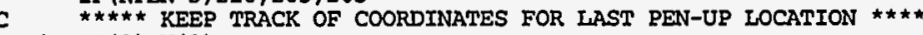

$\begin{array}{rl}205 & Y O(1) \\ \mathrm{XO}(2) & =\mathrm{Y}(1) \\ \mathrm{X}(2)\end{array}$

$\mathrm{YO}(2)=\mathrm{Y}$
$\mathrm{NPO}=0$

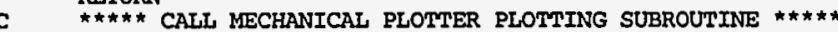

210 IF (NPO) 225, 220, 225

220 CONTINUE

225 CALT PLOT (YO (1), YO(2), 3 )

CALI PLOT (Y(1), Y(2), 2

RETURN

END

SUBROUTINE SEARC

DIMENSION NW(6),DX(3), SID (200), S2 (200), U(3),V(3),W(2,4), WW $(2,3)$

REAL^8 DZMIN, DZMAX, DZSTO, S1D, TD1, TD2, TD3 , TD4, D10K, D100K

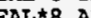

CIARACTER*4 TITLE, TITLE2

CHARACTER $* 8$ CHEM

$1, \operatorname{AIN}(140), \operatorname{ATOMID}(500), \operatorname{ATOMS}(3,500), \mathrm{BB}(3,3), \operatorname{BRDR}, \mathrm{CD}(8,20)$
$2, \operatorname{CONT}(5), \mathrm{D}(3,130), \mathrm{DA}(3,3), \mathrm{DP}(2,130), \mathrm{DISP}, \mathrm{EDGE}, \mathrm{FORE}, \mathrm{FS}(3,3,96)$

3 , IN, ITILT, KD $(5,20)$, LATM, NATOM, NCD, NJ, NJ2, NOUT, NSR, NSYM ،

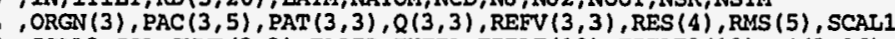

5 , SCAL2, SCL, SW V1 (4)

INTEGER *2 IDENTI

COMMON / PARMS/ CHEM $(505), \operatorname{EV}(3,505), \mathrm{P}(3,505), \mathrm{PA}(3,3,505)$

$1, \operatorname{IDENT}(2,505)$, MAXATM

logical featux

featur=. false

if ((ifeat.eq. 1 .or. ifeat.eq. 2) .and.

$(\bmod (n j 2,10)$.eq. 5 .or. $\bmod (n j 2,10)$

$\star \star \star \star \star$ OBTAIN

$\mathrm{D} 100 \mathrm{~K}=100000$

IF (NOUT. GE. O)

20 FORMAT (1HO $9 \mathrm{X}, 82 \mathrm{H}$

FROM ATOMS TO ATOMS WITH RADIUS OR SEMIDIMENSIONS $/ 11 \mathrm{X}, 46$

$\operatorname{IF}(\operatorname{ATN}(1)-D 10 K) 100,100,101$

100 ITOM1 $=$ AIN $(1)$

SYITOM $=5550$
GO TO 103

101 ITOM1 $=A I N(1) / D 100 \mathrm{~K}$

SYITOM=DMOD (AIN (1) D100K)

102 IF(DABS (AIN (2)) -Diok) 103,103,104

$103 \operatorname{ITOM2}=\operatorname{DABS}(\operatorname{AIN}(2))$

SYITO2 $=$ SYITOM

104 ITOM2=DABS (ATN (2))/D100K

SYITO2=DMOD (DABS (AIN (2)), D100K)

IF (ITAR1) 108, 108,110

08 ITAR1 $=1$

110 ITAR2 $=$ AIN (4)

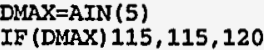

15 DMAX=4.

$\operatorname{ATN}(5)=D M A X$

$120 \mathrm{DMX}=\mathrm{DMAX} * \mathrm{DMAX}$

TEM $=.01$

$K F U N=N J * 100+M O D(N J 2,10)$

$\mathrm{K}=\mathrm{NJ} * 100+\mathrm{NJ} 2$

$I 0=S Y I T M$

LATOM=LATM

21 FORMAT (1H0, 10X, 2I3, I5 , I4 , I5, 2I4, 18X, 3F9.3/1H)

IF (NOUT.GE.0)

SWRITE (NOUT, 121)K, ITOM1, IO, ITOM2, I02

1ITAR1, ITAR2, (AXIN (J), J =5, 7 )

IF (NCD) $130,130,125$

25 IF (NCD) $130,130,1$

\&WRITE (NOUT, 124) ( $(\mathrm{KD}(J, I), J=1,4),(\mathrm{CD}(J, I), J=1,2), I=1$ $1 \mathrm{NCD})$

130 DO $135 \mathrm{~J}=1,4$

$135 \begin{aligned} & W(1, J)=99 \\ & W(2, J)=-99\end{aligned}$ 


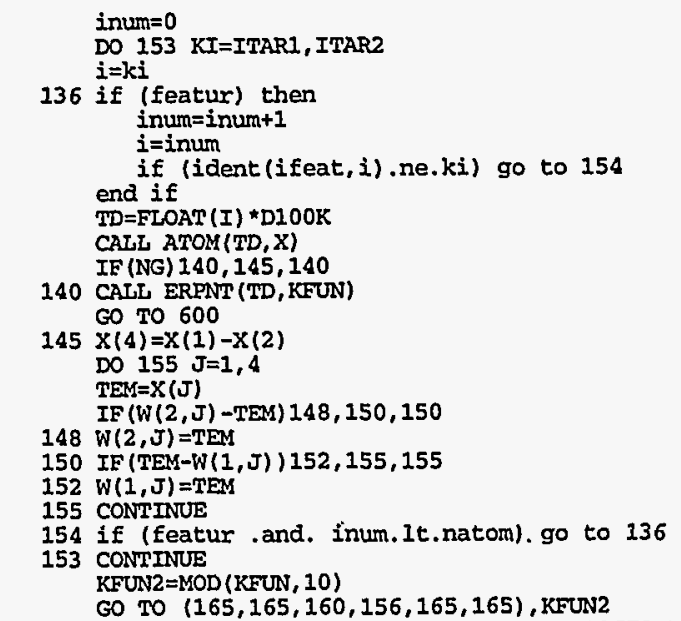

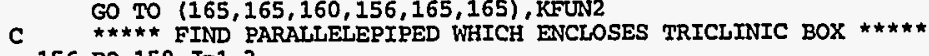
$156 \mathrm{DO} 158 \mathrm{~J}=1,3$
$158 \mathrm{DX}(\mathrm{J})=\operatorname{AIN}(\mathrm{J}+4)$

GO TO 170
$\star \star \star \star *$ FIND PARALLELEPIPED WHICH ENCLOSES RECTANGULAR BOX $\star \star \star \star \star$

160 DO $162 \mathrm{~J}=1,3$

$\operatorname{DX}(J)=0$. $162=1$

$162 \operatorname{TX}(J)=\operatorname{DX}(J)+A B S(R E F V(J, I) * T 9)$

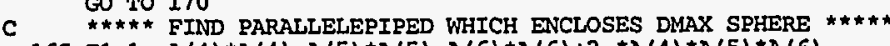

$165 T 1=1 .-A(4) \star_{A}(4)-A(5) \star_{A}(5)-A(6) \star_{A}(6)+2 . \star_{A}(4) \star_{A}(5) \star_{A}(6)$

DO $168 J=1,3$

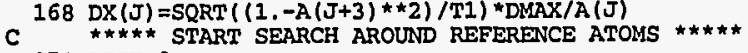

170 LIST $=0$

LAST $=0$
$M 1=I T O M 1$

$\mathrm{N} 1=$ ITOM2

C IF (KFUN2-5) 186, 172,172

C 172 IF (LATM) $174,174,176$

174 NG=12 FAULT, NO ENTRIB

$$
\text { GA TO } 600
$$

c $\begin{gathered}\text { G0 To* } \\ 176 \text { CHECK (LATM-LAST) } 600,600,177\end{gathered}$

177 LIST=LAST

178 LAST $=$ LATM

178 IF (LAST-LIST) $505,180,180$

180 TDI=ATOMID (LIST)

C $\star \star \star \star \star$ FIND SMALIEST ATOM NUMBER IN REMAINDER OF ATOMS LIST $* \star \star \star *$
LISTP1 $=$ LIST+1

DO $182 \mathrm{~J}=\mathrm{LISTP1}$, LAST

J)) GO To 182

Do $181 \quad I=1,3$

$\operatorname{ATOMS}(I, J)=\operatorname{ATOMS}(I$, LIST)

181 ATOMS (I, LIST) $=T 1$

TD1 $=$ ATOMID ( $J$ )

ATOMID (J) $=$ ATOMID (LIST)

ATOMID (LIST) $=$ TDD

182 CONTINUE
184 ITOM=TD1/D100K

if (featur)

if (featur) then

\& ident(ifeat, itom).gt.itom2) go to 178

else (ITOM.LT.ITOM1 .OR. ITOM.GT.ITOM2) GO TO 17

end if

SYITOM $=$ DMOD
SXITO2 $=$ SYITOM

\section{$M 1=I T O M$}

C $4 * \star \star \star$ SET INITIAI RUN PARAMETERS $\star \star \star \star \star *$

186 $M 2=A M O D$ (SYITOM, 100 .

$M 5=A M O D$ (SYITOM/100., 1000.)

$M 3=M 5 / 100$

C $* \star \star \star \star$ SET TERMINAI RUN PARAMETERS $* \star \star \star \star *$

$\mathrm{N} 2=\mathrm{AMOD}(\mathrm{SYITO2}, 100$.)

N5=AMOD (SYITO2/100., 1000.)

$\mathrm{N} 3=\mathrm{N} 5 / 100$

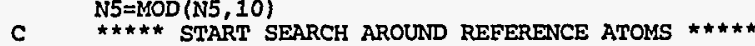

DO 500 L $5=\mathrm{M} 5$, N5

DO $500 \quad \mathrm{~L} 4=\mathrm{M} 4, \mathrm{~N}$

DO $500 \mathrm{~L} 3=\mathrm{M} 3, \mathrm{~N}$

DO 500 L2 $=M 2, N 2$

TD3=DBLE (ITOM) *D100K+DBLE (L3*10000+L4*1000+L5*100+L2)

CALL ATOM (TD3, $Y$ )

IF (NG) $188,190,188$

188 CALL ERPNT (TD3, KFUN)

GO TO 500
$\star \star \star \star \star$
$K=$ SMMAETRY EQUIVALENT POSITION $\star \star \star \star \star$

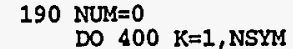

c DO $400 \mathrm{~K}=1$, NSYM DO $192 \mathrm{~J}=1,3$

$192 \mathrm{U}(\mathrm{J})=\mathrm{Y}(\mathrm{J})-\mathrm{TS}(\mathrm{J}, \mathrm{K})$

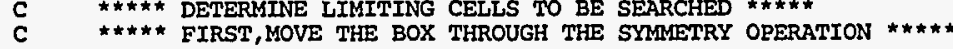
DO $200 \quad J=1,3$

DO $200 \mathrm{~L}=1,2$

$D O 200 \mathrm{I}=1,3$

DO $200 \quad I=1,3$

IF (TEM) $194,200,196$

194 N=MOD $(L, 2)+1$
GO TO 198 
$196 \mathrm{~N}=\mathrm{L}$

$W(L, J)=W W(L, J)+W(N, I) * T E M$

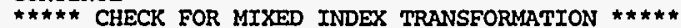

DO $215 \mathrm{~J}=1,2$

TEM $=F S(1, J, K)$

IF (TEM+ES $(2, J, K)) 215,201,215$

201 IF (TEM) $203,215,207$

\section{TW $(2, J)=W(1,4) \times D M$}

GO TO 215

$207 W W(1, J)=W(1,4) * T E N$

$W(2, J)=W(2,4) *$ TEM

c 215 CONTINUE

\#* MOVE 4 CELLS AWAY THEN MOVE BACK UNTTL PARALLELEPIPED AROUND $\mathrm{N}=0$

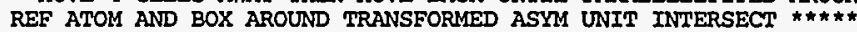

$\mathrm{N}=0$
DO $235 \mathrm{~J}=1,3$
DO $225 \mathrm{I}=1,2$

$\mathrm{N}=\mathrm{N}+1$

$T T=(U(J)-W W(I, J)) * F L O A T(I * 2-3)-D X(J)$

TF (TEM+TT) $225,225,221$

$225 \mathrm{NW}(\mathrm{N})=\mathrm{TEN} * \mathrm{FLOAT}(I \star 2-3)+5$

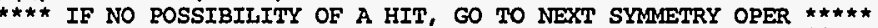
$F(\mathrm{NW}(\mathrm{N})-\mathrm{NW}(\mathrm{N}-1)) 400,235,235$

LL=NW(1)

$\mathrm{LU}=\mathrm{NW}(2)$

$M U=N W(4)$

$N \mathrm{~L}=\mathrm{NW}(5)$

$\mathrm{NU}=\mathrm{NW}(6)$

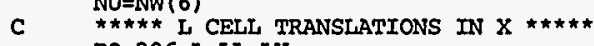

DO $396 \quad L=L L, L U$

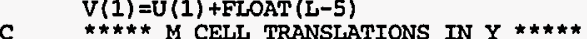

$396 \mathrm{M}=\mathrm{ML}, \mathrm{MU}$

$\mathrm{V}(2)=U(2)+\mathrm{FLOAT}(\mathrm{M}-5)$

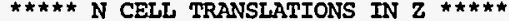

DO $396 \mathrm{NN}=\mathrm{NL}, \mathrm{NU}$

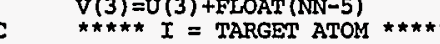

inum=0

$396 \mathrm{KI}=$ ITAR1, ITAR2

244 if (featur) then

inum $=$ inum +1

$i=$ inum

if (ident (ifeat, $i$ ).ne.ki) go to 395

end if

DO $250 \mathrm{~J}=1$,

DO 245 II $=1,3$

245 TEM $=T E M+E S(I I, J, K) \star P(I I, I)$

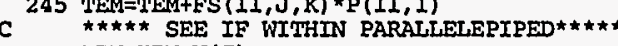
$T E M=T E M-V(J)$

IF (DX(J)-ABS (TEM)) $395,250,250$

TO $(255,255,252,265,255,255)$, KFUN2

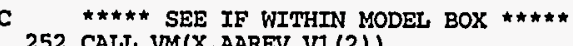

(J $+3 j-A B S(V 1(J))) 395,253,253$

253 CONTINUE

$$
\text { GO TO } 265
$$

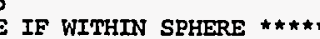

$255 \mathrm{DSQ}=\mathrm{VMV}(\mathrm{X}, \mathrm{AA}, \mathrm{X})$

(D)

(258

260 TEM $=5 O R T$ (DSO)

$\operatorname{IF}(\operatorname{AIN}(8)) 265,265,261$

C ${ }^{\star * \star * * \text { SELECT ONLY FIRST ASYMMETRIC UNIT ENCOUNTERED } * \star \star \star \star}$ 261 IF (LATM) 265, 265, 262

DZMAX

DO $264=1$, LATM

IF (DZSTO-DZMIN) 264, 263,263

263 IF (DZMAX-DZSTO) 264,264,395

264 CONTINUI

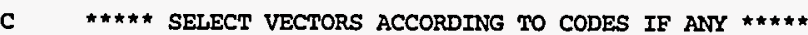

265 if (ncd.le.0) go to 277

c if logc $=0$, screening conditions are oRed

$\log c=\operatorname{ain}(8)$

8 DO $275 \mathrm{~J}=1, \mathrm{~N} C \mathrm{D}$

norg=itom

if $(\mathrm{kd}(5, j)$. eq. 1$)$ the

norg $=$ ident $(1$, itom $)$

ntar=ident $(1, i)$

end if

if $(\mathrm{kd}(5, j)$.eq. 2$)$ then norg=ident $(2$, i tom $)$

end if

flogc.eq. 0) then

$f(k d(2, j) . g t .0)$ then

if (norg.it.ka(1,j) .or. norg.gt.ka(2,j)) go to 275 end if

if $(k d(4, j)$. at 0$)$ then

if (ntar.It.kd $(3, j)$.or. ntar.gt.kd $(4, j))$ go to 275 end if if (tem.it.cd(1,j) .or. tem.gt.cd $(2, j))$ go to 275 end if

end if

(logc.eq.1) then

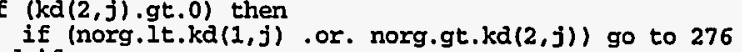
end if

if $(k d(4, j)$.gt 0$)$ then

if (ntar.it.ka $(3, j)$.or. ntar.gt.kd(4,j)) go to 276 end if $(2, j)$.gt. 0. ) then

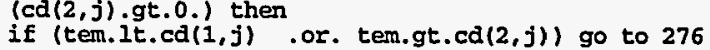




\author{
end if
if (j.eq.ncd) go to 277 \\ 275 CONTINUE
}

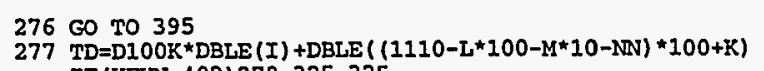

c IF (KFUNN-402) 278, 325, 325

278 IF (NON) 317, 317, 279

TT $=S 2$ (II) $-T E M$
IF (ABS (TT) -0.0001$) 297,297,281$

281 IF (TT) $315,297,283$

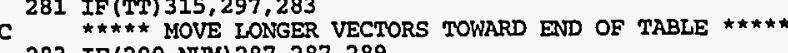

283 IF (200-NUM) 287, 287, 289

287 NUM $=199$
289
I $J=N U M$

DO $295 \mathrm{~J}=\mathrm{II}, \mathrm{NUM}$
$\operatorname{SID}(I J+1)=\operatorname{SID}(\mathrm{IJ})$

$\mathrm{S} 2(I J+1)=\mathrm{S} 2(\mathrm{IJ})$

295 IJ=IJ-1

C 297 CALL ATOM(S1D(II) 7 )

DO $305 \mathrm{~J}=1,3$

305 CONTINUE $D(I I), Z)$

GO TO 395

315 CONTINUE

317 II $I N=N M+1$
319

319 NUM=NOM+1

$$
\begin{aligned}
& \text { S1D (II) }=\text { TD } \\
& \text { S2 (II })=T E M
\end{aligned}
$$

$320 \mathrm{IF}(\mathrm{KFUN}-106) 395,325,325$

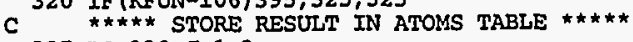

$325 \mathrm{D} 330 \mathrm{~J}=1,3$

V1 $(J)=X(J)+Y(J)$

395 if (featur . and. Inum.1t.natom) go to 244

396 CONTINUE

C 400 CONTINUE

421 FORMAT (1HO10X, 2OHVECTORS FROM ATOM (I3, 1H, I5, 1H) 6X, 8HTO ATOMSI 4 , $18 \mathrm{H}$ THROUGH, I4

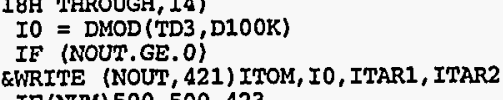

IF (NUM) 500,500 ,

$$
\begin{aligned}
& \text { TD2 }=S 1 D(I) \\
& \text { I1 }=\text { TD2 } / D 100 K \\
& \text { I2=TD2-DBLE (II) *D100K } \\
& \text { CALL ATOM (TD2, Z) } \\
& \text { IF (I-1) } 432,432,434
\end{aligned}
$$

IF(I-1) 432,432,434 $1 \mathrm{X}), 39 \mathrm{X}, 1 \mathrm{H}(\mathrm{I3}, 1 \mathrm{H}, \mathrm{I5}, 1 \mathrm{H}) 3 \mathrm{F7} .4,7 \mathrm{X}, 3 \mathrm{HD}=\mathrm{F} 6.3)$

427 FORMAT $(1 \mathrm{H} 13 \mathrm{X}, 2(\mathrm{AG}, 1 \mathrm{X}), 39 \mathrm{X}, 1 \mathrm{H}(\mathrm{I} 3,1 \mathrm{H}, \mathrm{IS}, 1 \mathrm{H}) 3 \mathrm{FF} .4,7 \mathrm{X}, 3 \mathrm{HD}=\mathrm{F} 6.3)$
429 FORMAT $(1 \mathrm{H}) 13 \mathrm{X}, 2(\mathrm{AG}, 1 \mathrm{X}), 2(3 \mathrm{H}(\mathrm{I3}, 1 \mathrm{H}, \mathrm{I5}, 1 \mathrm{H}) 3 \mathrm{~F} 7.4,3 \mathrm{X}), 4 \mathrm{X}, 3 \mathrm{HD}=\mathrm{F} 6.3)$

432 IF (NOUT.GE.O)
QWRITE (NOUT, 429) CHEX (ITOM), CHEM(II), ITOM, IO, (Y (J) , J=1, 3 1), I1, I2, $(Z(J), J=1,3), S 2(I)$

434 IF (NOUT.GE.0)

\&WRITE (NOUT, 427) CHEM(ITOM), CHEM(I1), I1, I2, (Z (J),J=1,3), $1 S 2$ (I)

c 435 CONTINUE

437 (XFUN-102) $500,451,500$

441 FORMAT (1HO, 10X,18HANGLES AROUND ATOM, I5)

451 IF (NOUT.GE. 0)
SWRITE (NOUT, 441) ITOM $L=N U M-1$

57 (1) $500,500,457$

(5) $465 \quad I=1$,

$T 22=S 1 D(I)$

$\mathrm{II}=\mathrm{TD} 2 / \mathrm{D} 100 \mathrm{~K}$

$I 2=T D 2-D B L E(I 1) * D 100 K$

CALI ATOM (TD2, $X)$

CALI DIFV $(X, Y, U)$
CALL $M V(A A, U, V 2)$

$M=I+1$

$D O \quad 465 J=M, N U M$

$\mathrm{TD} 4=\mathrm{S} 1 \mathrm{D}(\mathrm{J})$

$\mathrm{J} 1=\mathrm{TD} 4 / \mathrm{D} 100 \mathrm{~K}$

$\mathrm{J} 2=T \mathrm{DD} 4-\mathrm{DBLE}(\mathrm{J} 1) * \mathrm{D} 100 \mathrm{~K}$

CALL ATOM(TD4, $Z$ )

$F=\operatorname{ARCCOS}(\mathrm{VV}(\mathrm{V}, \mathrm{V} 2) /(\mathrm{T} 3 * \mathrm{~S} 2(\mathrm{~J})))$

$\operatorname{CALL} \operatorname{DIFV}(x, z, V 3)$

$\mathrm{FI}=\mathrm{SQRT}(\mathrm{VMN}(\mathrm{V} 3, \mathrm{AA}, \mathrm{V} 3))$
$460 \operatorname{FORMAT}(1 \mathrm{H} 13 \mathrm{X}, 3(\mathrm{~A} 6,1 \mathrm{X}), 7 \mathrm{X}, 3(2 \mathrm{H}(\mathrm{I} 3,1 \mathrm{H}, \mathrm{I5}, 1 \mathrm{H})), 12 \mathrm{X}, 3 \mathrm{HD}=\mathrm{F} 6.3,7 \mathrm{X}, 3 \mathrm{HA}$ $1=\mathrm{F} 6.2)$

IF (NOUT.GE.0)

QWRITE (NOUT, 460) CHEM (I1), CHEM (ITOM), CHEM (J1), I1, I2, ITOM

$1, \mathrm{I} 0, \mathrm{~J} 1, \mathrm{~J} 2, \mathrm{~F} 1, \mathrm{~F}$

465 CONTINUE

495 CONTINUE

IF (LAST-LIST) 505, 505, 178

S05 IF (KFUN2-6) 600, 176,600

605 LATM=LATOM

END

SUBROUTINE SIMBOL (W, W2, HGT, ITXT, THT, N)

DIMENSION W(3), ITXT (72),DS(10),DC(10)

DIMENSION IPR (64), Manc(64), 1 ,

CHARACTER*1 ITXT

common /ns/ npf, ndraw, norient, nvar

common /trfac/ xtrans, ytrans

DATA IFTR

$1 / 312,32,41,54,47,20,20,54,64,70,103,108,27,115,118,128$ $2,140,128,140,194,166,102,208,120,27,211,216,0 ， 0 ， 0 ， 0,237$ $3,0,150,328,301,168,223,92,328,88,188,180,1,82,180,82,30$ $5,350,360,370,378,388,398,407,421,428,436,444,450,455,465,472,484$ $6,494,504,510,521,526,533,536,541,546,550$ DATA NKNT 


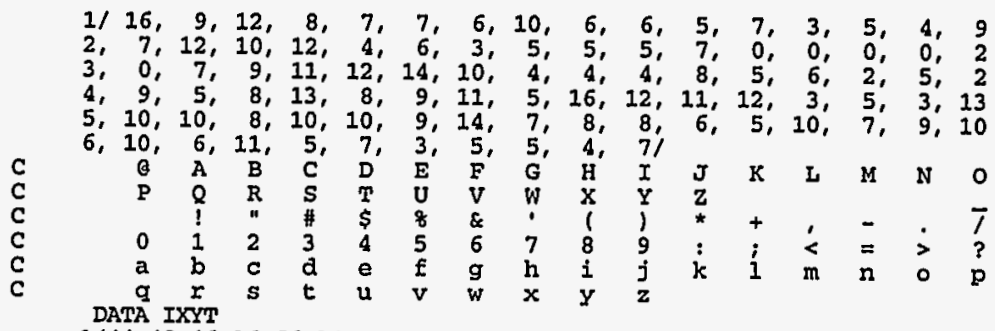

DATA IXYT

$1 / 44,48,46,26,66,24,64,99,66,26,68,26,64,24,66,28,29,22,62,69$ $2,29,26,56,26,22,62 ; 29,62,99,22,69,22,25,65,25,28,39,59,68,62$ $4,63,5655$

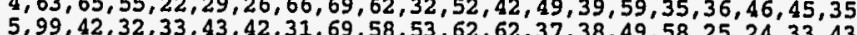
$6,64,29,23,32,52,63,69,29,22,25,69,99,47,62,22,29,45,69,62,49$ $7,22,46,62,69,47,69,99,68,59,39,28,23,32,52,63,68,99,44,62,22$ $8,29,59,68,67,56,26,56,65,62,49,44,99,32,43,52,32,99,44,46,56$ $9,67,68,59,39,28,29,69,49,42,99,23,53,64,65,56,36,27,38,68,25$ A, $C, 36,56,38,28,29,39,38,99,69,22,99,53,63,62,52,53,15,75,32,9$

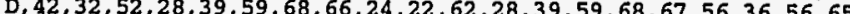
$E, 63,52,32,23,28,39,59,68,29,24,64,54,59,52,42,62,23,32,52,63$ $F, 65,56,25,29,69,68,43,42,23,32,52,63,68,59,39,28,26,35,55,66$ $\mathrm{G}, 24,64,54,53,57,56,66,26,36,37,33,66,57,47,36,35,44,54,65,67$
$\mathrm{H}, 58,38,27,24,33,53,64,57,49,59,57,99,37,29,39,37,22,32,12,22$ c 349

$a, 62,67,66,57,37,26,23,32,52,63$
$b, 22,29,26,37,57,66,63,52,32,23$

$c, 63,52,32,23,26,37,57,66$

e, 63,52,32,23,26,37,57,66,65, 35

c 420

$64,67,66,57,37,26,24,33,53,64,62,51,31,22$

$\mathrm{h}, 22,29,26,37,57,66,62$

$i, 32,52,42,47,37,99,48,49$
$j, 32,41,51,62,67,99,68,69$

$x^{2}, 22,29,24,57,35,62$

$\mathrm{m}, 22,27,26,37,46,42,46,57,66,62$

c 458

$n, 22,27,26,37,57,66,62$
$0,63,52,32,23,26,37,57,66,63,99,67,45$

p, $21,27,26,37,57,66,64,53,33,24$ $\frac{9}{4}, 61,67,66,57,37,26,24,33,53,64$

$5,23,32,52,63,64,55,35,26,37,57,66$

$\mathrm{t}, 42,48,46,36,56$

$u, 67,62,66,52,32,23,27$

C

$w, 27,32,46,52,67$ $y, 27,43,67,31$

$Y, 27,42,31,42,67$

, $22,22,67,27,99,35,55$,

if (ndraw eq.

write (npE, 21) n, nvar, w(1) +xtrans, w(2) +ytrans, 8, *hgt, tht

21 format (' $\left.T X T^{\prime}, 12,1 x, 12,{ }^{\prime} 11^{\prime}, 4(1 x, f 10.6)\right)$

write (npf, 22) (itxt(k), $k=1, n)$

return

end

IF (N.LE.0) GO TO 400

C----SET UP TABLE OF INCREMENTS BASED ON HGT AND THT IF (THT.EQ.0.0) GO TO 120

TH=RAD*THT
ST=SIN (TH)

$\mathrm{CT}=\cos (\mathrm{TH})$

$120 \mathrm{ST}=0.0$

CT $=1.0$

$130 \mathrm{D}=\mathrm{HGT} / 7.0$

$\mathrm{DST}=\mathrm{D} * \mathrm{ST}$

$D C T=D^{*} C T$

$D S(1)=-D S T$
$D C(1)=-D C T$

DO $145 \quad I=2,10$

$D S(I)=D S(I-1)+D S T$

145 CONTINUE

$\mathrm{YO}=0.0$

DO $370 \mathrm{~J}=1, \mathrm{~N}$

IXTU $=$ ICHAR (ITXTT (J)

If (itxtj.ge.97.and.1txtj.1e.122) then ich=it xtj -32
go to 221

and $i$

C-D-MASK IT TO SIX BITS AND ADD ONE. PICK UP POINTER AND COUNTER

IP=IPTR (ICH)

$N K=$ NKNTT (ICH)

C----TEST FOR SPACE OR UNDEFINED CHARACTER

IF (NK.ER.0) GO TO 360

SEGMENTS OF CHARACTER. LIFT PEN INITIALLY

DO $350 \mathrm{~K}=1$, NK

C----LIFT PEN IF SPECIAL INDICATOR IS FOUND

IF (IXY.NE.99) GO TO 300

IPEN $=3$

300 IX $=\mathrm{TXY} 340$

IY $=$ IXY $-10 *$ IX

C (IX) $-D S(I Y)$
DC IY $+D S(I X)$

(IX)

CALL DRAW (W, DX, DY, IPEN)
C---PUT PEN DOWN TO DRAW NEXT SEGMENTS 
340 IP $=\mathrm{IP}+1$

350 CONTINUE

$360 \mathrm{XO}=\mathrm{XO}+\mathrm{DC}(8)$

$\mathrm{YO}=\mathrm{YO}+\mathrm{DS}(8)$

370 CONTINUE

--PLOT ONE SRECIFIC CENTERED SYMBOL. SET UP TABLE OF INCREMENTS DCT=HGT $/ 2.0$

$D C(1)=-D C T$

C----MOVE TO SYMBOL WITH PEN UR OR DOWN, DEPENDING ON$$
\text { IPEN }=3
$$

IF (N. LE. -2) IPEN=2
C-----LOOP THROUGH SEGMENTS OF CENTERED SYMBOL

$\operatorname{IXY}=\operatorname{IXXT}(\mathrm{K})$

$I Y=I X Y-10 * I X$

C-----PUT PEN DOWN TO DRAW REMAINING SEGMENTS

IPEN $=2$

440 CONTINUE

SUBROUTINE SPARE(INST)

AHIS SUBROUTINE MAY BE USED FOR NEW INSTRUCTIONS $* * * *$ RETURN

END

TTER (T⿰亻)

C REAL*8 TDI 1

REAL*8 AIN, ATOMID

CHARACTER *4 TITLE, TITLE

COMMON NG, $A(9), \operatorname{AA}(3,3), \operatorname{AAREV}(3,3), \operatorname{AAWRK}(3,3), \operatorname{AID}(3,3)$

$1, \operatorname{AIN}(140), \operatorname{ATOMID}(500), \operatorname{ATOMS}(3,500), \mathrm{BB}(3,3), \operatorname{BRDR}, \mathrm{CD}(8,20)$

,

,

, SCAL 2 SCL, SYM (3) 3) TAPER THETA, TITLE (18), TITLE2 (18) TS $(3,96)$

$6, \operatorname{VIEN}, \operatorname{VT}(3,4), \operatorname{V1}(4), \operatorname{V2}(3), \operatorname{V3}(3), \operatorname{V4}(3), \mathrm{V} 5(3), \mathrm{V} 6(3), \operatorname{WRKV}(3,3)$

,XLNG (3), XO(3),XT(3)

IF (LATM) 481, 481,450

450 IF (500-LATM) $455,455,460$

$460 \mathrm{~L}=\mathrm{LATM}$

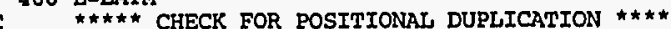

DO $480 \quad K=1, L$

$\operatorname{IF}(A B S(V 1)(J)$-ATOMS $(J, K))-0.001) 465,465,480$

65 CONTINUE

IF (NJ2-10) 490,490,470

***** ATOM REMOVAL BY TABLE PUSHDOWN $* * \star \star *$

LATM=LATM-1

$\operatorname{ATOMID}(I)=\operatorname{ATOMID}(I+1)$

DO $475 \mathrm{~J}=1,3$

475 ATOMS $(\mathrm{J}, \mathrm{I})=$ ATOMS $(\mathrm{J}, \mathrm{I}+1)$
GO TO 490
480 CONTINUE

481,400

482 IF (499-LATM) 490,483,485

CALL ERPNT (TD1, 400 )

485 LATM $=\mathrm{LATM}+1$

DO $486 \quad J=1,3$
ATOMS $(J$, LATM $)=V 1$ (J)

ATOMID (LATM) $=$ TDI

490 RETURN

subroutine tepsym (tot, num, kk)

c $\star * \star$ parses character string representation of symmetry operators

*** and stores information

REAL *8 AIN, ATOMID

CITLE, TITLE2

ATN(140) ATOMrD $(3,3), \operatorname{AAREV}(3,3), \operatorname{AAWRK}(3,3), \operatorname{AID}(3,3)$

$2, \operatorname{CONT}(5), \mathrm{D}(3,130), \mathrm{DA}(3,3), \mathrm{DP}(2,130), \mathrm{DISP}, \operatorname{EDGE}, \operatorname{FORE}, \operatorname{FS}(3,3,96)$

$4, \operatorname{ORGN}(3), \operatorname{PAC}(3,5), \operatorname{PAT}(3,3), Q(3,3), \operatorname{REFV}(3,3), \operatorname{RES}(4), \operatorname{RMS}(5), \operatorname{SCAL} 1$

5 . SCAL2, SCL, SMBB

logical twodig

$c * * * \begin{gathered}\text { convert txt to upper case } \\ \text { do } 101 \text { i } i=1,24\end{gathered}$

iascili=ichar(tot ( $i \cdot i)$

if (iascii.ge.97.and.iascii.le.122) $\operatorname{txt}(i: i)=\operatorname{char}($ iascii-32)

*** default value of ts if no fraction specified

202 ts $(k k$, num $)=0$

$c * \star \star$ look for and interpret $a / b$ style fraction $n=1$ ndex (txt, in

get denominator

$k=1 \operatorname{char}(\operatorname{txt}(n+1: n+1))-48$

m=ichar (txt $(n+2: n+2))-48$ then

if (m.ge. 0 iden $k * 10+m$

else
iden=k
end if

c get numerator

twodig=. false

$k \sin =1$

$k=1 \operatorname{char}(\operatorname{txt}(n-1: n-1))-48$

$m=i \operatorname{char}(\operatorname{txt}(n-2: n-2))-48$

if (m.ge.0 and. m.le.9) twodig=, true.

if $(n-3,(n-2: n-2)$.eq. -1$)$ ksign=-1

if (txt $(n-3: n-3)$.eq. ' -1$)$ ksign=-1 


$$
\begin{aligned}
& \text { end if } \\
& \text { if (twodig) then } \\
& \text { inum=ksign * }(\mathrm{m} \cdot 10+k) \\
& \text { else } \\
& \text { inum=ksign * } k \\
& \text { end if }
\end{aligned}
$$

ts (kk, num)
end if float (inum) /float (iden)

c*** look for and interpret decimal style fraction if (n.gt.0) then

c get post decimal point portion $k=i \operatorname{char}(\operatorname{txt}(n+1: n+1))-48$
$m=i \operatorname{char}(\operatorname{txt}(n+2: n+2))-48$ m=ichar (txt $(n+2: n+2))-48$ if $(m . g e .0$.and. m.le.9) then else $(k k$, num $)=$ float $(k) * .1$

end if

c get sign

$$
\begin{aligned}
& \text { ksign=1 } \\
& \text { if (n-1.ge.1) then } \\
& \text { if (txt(n-1:n-1). eq. '- ') ksign=-1 } \\
& \text { end if } \\
& \text { if (n-2.ge.1) then } \\
& \text { if (txt (n-2:n-2). eq. '- - ) ksign=-1 } \\
& \text { end if } \\
& \text { ts (kk,num)=float (ksign) * ts (kk, num) } \\
& \text { end if }
\end{aligned}
$$

$c * * *$ interpret $x y z$ portion of symmetry operation

$$
\text { do } 303 \quad i=1,24
$$

if $(\operatorname{txt}(i: i)$.eq. ' $x$ ') then fs $(1, k k$, num $)=1$.

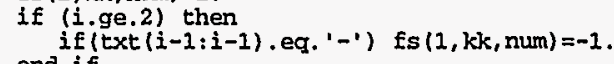
end if

(txt $(i: i)$. eq. ' $Y$ ') then fs $(2, k k$, num $)=1$.

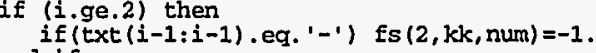
end

if (txt(i:i).eq.' $z$ ') then $f s(3, k k, n u m)=1$. if $(i, g e .2)$ then
if $($ txt $(i-1: i-1)$.eq. '-') fs $(3, k k$, num $)=-1$.
end if

303 end if

\section{return}

c $\quad \mathrm{Z}=$ TRANSPOSED (TRANSPOSE $(\mathrm{X}) \cdot(\mathrm{Y})$ )
C $\quad \mathrm{Z}(3,3)=\mathrm{X}(3,3) * \mathrm{Y}(3,3)$

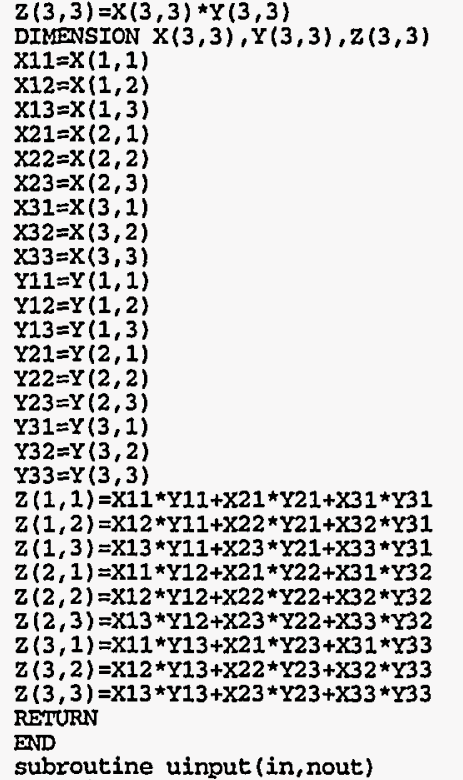

subroutine uinput (in, nout)

$c * * *$ user input routine

ns/ npf ndraw, norient, nvar

comon /dfl/ infile, iäraw, iorient, iout, ext, atomfi, fpaplen character $* 60$ fname, txtans, infile, atomfi

character $* 4$ ext
character*1 answer

call dflts

$c * *$ get the input file name and open the file or "exit" $* \star *$

if (iargc ().eq. 1) then

call getarg (1, fname) open (in, fi
go to 135

end if

110 fname=infile

ipos=index (fname, ' ')
write $(*, 115)$ fname (1:ipos-1)

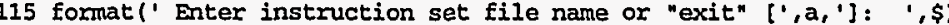
read $(*, 120)$ txtans

120 format (a)

if (txtans (1:4) .eq. 'exit' .or. txtans (1:4) .eq. 'ExIT' ) if (totans (1:1) if (Extans (1:1) . ne. ' ' ') fname=textans

go to 135

125 ipos=index (fname, ' ')

write $(*, 130)$ fname $(1:$ ipos -1$)$ 
130 format (/, " ", $a, "$ " does not exist"/)

go to 110

$c * * *$ determine where oxtep drawing should go ***

135 write $(*, 140)$ idraw *, or (0) Omit $\left[{ }^{1}, 11,1\right]$ : Screen, (2) Postscript file, (3) HPGL file

145 format (a)

if (answer .eq. ' ') then

ndraw=idraw

else

read (answer, 155) ndraw

end if

(ndraw. 1t. 0.or. (ndraw.gt. 3. and.ndraw.ne.9)) then

write $(6 *)$ invalid selection

go to 135

o. or.ndraw. eq.1.or.ndraw. eq.9) go to 149

if (ndraw

$c * * *$ need to get this information if printing from editor entry getpap

$c \star \star \star$ determine orientation of drawing ***

1451 write $(*, 1452)$ iorient

1452 format (" (1) Portrait or (2) Landscape orientation $[1, i 1,1]$ : $1, \$)$

if (answer. eq. "') then

norient=iorient

end if

if (norient.1t.1.or norient.gt. 2) then write $(6, *)$ invalid selection go to 145

$c * \hbar *$ determine paper length for postscript landscape

if (ndraw.eg.2. and.norient. eq.2) then

1453 format (' How tall is printer page in inches? $[1, f 5,2,1]:$, , \$ read ( $\left.{ }^{*}, 120\right)$ txtans $($ txtans $(1: 1)$.ne. I) read (txtans, 1454) fpaplen

1454 format $(\mathrm{E} 10.0)$ end if

$c * * *$ if called from recycle, return there

if (iflag.eq.1) return

$c * * *$ determine where ortep output should go $* *$

149 write $(*, 150)$ lout

format (' Text output to (1) File, (2) Screen, or (0) Omit $[1$, il, (1) : read $(*, 145)$ answer nou=iout

$$
\text { else }
$$

read (answer, 155) nou

end if

$c$ 155 format (i1)

nout $=-4$

if (nou .eg. 1) nout $=4$
if (nou .eq. 2) nout $=6$

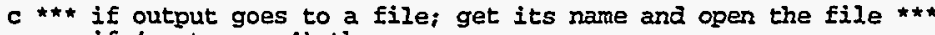

if (nout. eq. 4) then

ipos=index (Ename, ' $'$ ')

fname $=$ fname $(1$ : ipos-1)//ext

go to 160

ipos=index (fname, ' ')

160

ipos=iname (1:ipos-1)//ext

write $(*, 165)$ Ename $(1$ : ipos-1)

format ( Enter output file name $\left[{ }^{\prime}, a,{ }^{\prime}\right]:$, , \$)

if (txtans (1.1) ne.

1) fname=txtans

open (nout, file = fname, status=' old' , exr $=170$ )

170 open if

175 continu

(nile=fname, status= ' new' )

c *** get file name of an external file containing atomic parameters

210 entry gtafil (iul

ipos=index (Ename, ' ')

write $(*, 215)$ fname (1:ipos-1)

215 format (' Enter atom parameter file name or "exit" $\left[{ }^{\prime}, a, l\right]$ :,$\$$ read $(*, 120)$ extans

'exit' .or. txtans (1:4) .eq. 'ExIT')

if (txtans $(1: 1)$.ne. ' ) fname=txtans

225 ipos=index (fname, ' ')

rite $(\star, 130)$ fname (1: ipos-1)

*** ask user about using editor

entry go2eatr

c 303 format $(1,1$ Edit instruction set? (Y)es or (N)० [N]: ', \$)

03 format $(, \cdot$ (1) save drawing as Postscript file, 1 ,

\&' (3) Redraw structure on screen',';

' (4) Edit instruction set', 1

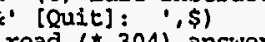

304 format (a)

if (answer, eq. '1') then

naraw $=2$

call recycle

ise

(answer.eq. '2') then ndraw $=3$

call recycle

if (answer. eq. '3') then ndraw $=1$

call recycl

if (answer.eq. '4') call editr

end if 
end if

end

SUBROUTINE UNITY $(X, z$, ITYPE)

REAT*8 ATN

CHARACTER *4 TITIE

COMMON NG, $\operatorname{A}(9), \operatorname{AA}(3,3), \operatorname{AAREV}(3,3), \operatorname{AANRK}(3,3), \operatorname{AID}(3,3)$

, AIN (140), ATOMID (500), ATOMS $(3,500), B B(3,3), \operatorname{BRDR}, \operatorname{CD}(8,20)$

$, \operatorname{CONT}(5), D(3,130), D A(3,3), D P(2,130), D I S P, E D G E, F O R E, F S(3,3,96)$

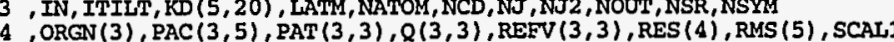

,ORGN (3), DAC ,

6 , VIEN, VT $(3,4), \mathrm{V} 1(4), \mathrm{V} 2(3), \mathrm{V} 3(3), \mathrm{V} 4(3), \mathrm{V} 5(3), \mathrm{V} 6(3), \mathrm{WRKV}(3,3)$

XING (3), XO(3), XT (3)

$\mathrm{Y}(1)=\mathrm{X}(1)$
$\mathrm{Y}(2)=\mathrm{X}(2)$

$\mathrm{Y}(3)=\mathrm{X}(3)$

(ITYPE) $125,125,105$

$05 \mathrm{~T} 1=\operatorname{SQRT}(Y(1) * Y(1)+Y(2) * Y(2)+Y(3) * Y(3))$

T1 $=S O R T(Y(1) *(Y(1) * A A(1,1)+Y(2) *(A A(1,2)+A A(2,1))+Y(3) *(A A(1,3)+A$ $1 A(3,1)))+Y(2) *(Y(2) * A A(2,2)+Y(3) *(A A(2,3)+A A(3,2)))+Y(3) * Y(3) \star A A(3$

145 IF (T1) $155,155,175$

(1) 300

$175 \mathrm{Z}(1)=\mathrm{Y}(1) / \mathrm{T}$

$Z(2)=Y(2) / T 1$

00 RETURN

SUBROUTINE VM $(Y, X, Z)$

ECTOR * MATRIX

$(3)=Y(3) * X(3,3)$

$\mathrm{Y} 2=\mathrm{Y}(2)$

$\mathrm{Y} 3=\mathrm{Y}(3)$

$Z(1)=X(1,1) * Y 1+X(2,1) * Y 2+X(3,1) * Y 3$

$Z(3)=X(1,3) * Y 1+X(2,3) * Y 2+X(3,3) * Y 3$

RETURN

FUNCTION VMV $(\mathrm{X} 1,0, \mathrm{X} 2)$

C TRANSPOSED VECTOR * MATRIX * VECTOR

TO EVALUATE QUADRATIC OR BILINEAR FORM

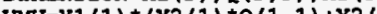

$+\times 1(2) *(\times 2(1) * 0(2,1)+\times 2(2) * Q(1,2)+X 2(3) * Q(1,3))$

$+\mathrm{X} 1(3) *\left(\mathrm{X}_{2}(1) * \mathrm{Q}(3,1)+\mathrm{X}_{2}(2) * \mathrm{Q}(3,2)+\mathrm{X} 2(3) * \mathrm{Q}(3,3)\right.$ RETUR

ENDNCTION $V W(X, Y)$

C $\quad \mathrm{VV}=\mathrm{X}(3) \approx \mathrm{Y}(3)$

DIMETSTON $X(3), y(3)$

$\mathrm{V}=\mathrm{X}(1) \star Y(1)+X(2) \star Y(2)+X(3) \star Y(3)$

RETURN

SUBROUTINE XYZ (DQA, $X$, ITYPE)
C $\star \star \star \star *$ ITYPE GT. G CART. COORD. FROM ATOM CODE WORD $* * \star \star *$

*t*** XABSF (ITYE) .LE.2 FOR WORKNG STSTEM

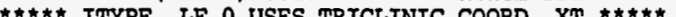

DIMENSION $X(3)$

AL"8 AIN, ATOMID

COMMON NG, A $(9), \operatorname{AA}(3,3), \operatorname{AAREV}(3,3), \operatorname{AANRK}(3,3), \operatorname{AID}(3,3)$

1 , AIN (140), A(

4 ORGN (3), PAC $(3,5), \operatorname{PAT}(3,3), O(3,3)$, REFV $(3,3), \operatorname{RES}(4), \mathrm{RKS}(5)$, SCAL1

5 ,'SCAL2, SCL, SYMB $(3,3)$, TAPER, THETA, TITLE (18), TITLE2 (18), TS $(3,96)$

$6, \operatorname{VIEN,VT}(3,4), \operatorname{V1}(4), \operatorname{V2}(3), \operatorname{V} 3(3), \mathrm{V} 4(3), \mathrm{V} 5(3), \mathrm{V} 6(3), \operatorname{VRKV}(3,3)$

$7, \mathrm{XLNG}(3), \mathrm{XO}(3), \mathrm{XT}(3)$

I $T=$ IABS (ITYPE)$$
\begin{aligned}
& N G 1=N G \\
& N G=0
\end{aligned}
$$

IF (ITYPE) $10,10,5$

CALL ATOM(DQA, XT

$10 \mathrm{~T} 1=0$.

DO $15 \mathrm{~J}=1,3$

T2 $=$ XT (J) $-O R G N(J)$

15 T1=Ti+ABs

IF (T1-.0001)20,20,40

$20 \mathrm{NG}=\mathrm{NG1}$

$30 \mathrm{DO} 35 \mathrm{~J}=1,3$

$35 X(J)=0$.

$40 \mathrm{IF}$ (IT) $45,45,60$

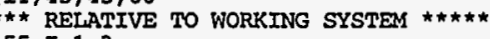

$45 D 5 \quad I=1,3$

$50 \mathrm{~T} 1=\mathrm{T} 1+\mathrm{V} 1(\mathrm{~J}) * \operatorname{AAWRK}(\mathrm{J}, I)$

S5 $X(I)=T I * S C A L$

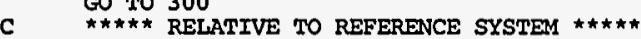

DO $70 \mathrm{I}=1,3$

DO $65 \mathrm{~J}=1,3$

$65 \mathrm{~T} 1=\mathrm{T} 1+\mathrm{V} 1(\mathrm{~J}){ }_{\mathrm{AAREV}}(\mathrm{J}, \mathrm{I})$

$70 X(I)=T 1 * S C A L$

\section{c} c $\star \star \star$ DUMMY SCREEN OUTPUT (MAY BE REPLACED WITH SCREEN DRIVER CODE)

subroutine inits return

subroutine penwsc(penw)

return
end

subroutine colrsc(icolor) 
c return

c subroutine pensc $(x, y$, ipen $)$

return

c subroutine endsc

$$
\text { return }
$$

$c * \star \star$ end of dummy screen output

c

\section{$c * * *$ PGPLOT CODE FOR SCREEN OUTPUT}

$c * \star *$ if PGPLOT is implemented, use the subroutines here

$c * *$ instead of the ones in the DUMMY SCREEN OUTPUT section PGPLOT is a Eree graphics library developed by $T$. J. Pearson at about PGPLOT can be found on the World wide Web a

c or via e-mail to tjpeastro.caltech. edu.

subroutine initsc

character in the

integer pgbeg

xwid $=11$.
yhgt $=8.5$

$c * *$ The following is for PGPLOT on an $\mathrm{X}$-windows system.

$c * \star \star$ The following is for PGPLOT on an MS-DOS system.

c outdev $=1 /$ MS

$c \star \star \star *$ The following is for PGPLOT on a Macintosh system. outdev $=1 /$ MAC

open (npf, status= ' scratch')

if (pgbeg $(0,1,1,1)$.ne. 1) call exitng(8)

c switch black and white

call pgscr $(0,1 ., 1 ., 1)$

c set up drawing window
call pgpage
call pgqch (osize)
call pgsch $(0$.
call pgwnad( 0 , , swid, $0 .$, yhgt
call pgsch (osize)
$0, \cdot \mathrm{BCT} \cdot 1,1,0\rangle$
call pgsfs (2)
call pgrect $(10,4,11,1,8.2,8.5)$
call pgtext $(10.5,8,3, \cdot \operatorname{ExIT} i)$

return

subroutine colrsc(icolor)

*** set plot color

$=0 \Rightarrow$ black

*** PGPLOT is set up for $1=b l a c k$

common /ns/ npf, ndraw, norient, nvar

if (icol.eq. 0 ) $i c 01=1$

if (ndraw. eq.1) call pgsci(icol)

11 if (ndraw.eq.9) write (npf,111) icol return

\section{subroutine penwsc (penw)}

$c * * *$ PGPDOT peasures pen wiath in 200ths of an inch common /ns/ npf, ndraw, norient, nvar

ipenw=nint $(.001 *$ penw*200.

if (ipenw. 2e. 0) ipenw=1

if (ipenw.gt.200) ipenw $=200$

if (ndraw.eq.1) call pgslw(ipenw)

111 format ('WID', 1x, i3)

return
end

subroutine penșc ( $x, y$, ipen)

move the pen

xtrans, ytrans

conmon /ns/ npf, ndraw, norient, nvar

if (ipen.eq. 2) then

if (ndraw.eq.

if (ndraw.eq.9) write (npf,111) $x+x$ trans, $y+y$ trans

111 format ('LIN',2(1x, 110.6$))$

en.eq. 3) then

(ndraw. eq.1) call pgmove ( $x+x$ trans, $y+y t r a n s$ )

112 format ('MOV'.2(1x, f10.6))

retur

subroutine endsc

common /ns/ npf, ndraw, norient, nvar

call curssc
close (npf)

$c \star \star \star$ tell user to hit <enter> key

call pgsci $(0)$

call pgrect $(7,5,11,8,2,8,5)$

call pgsci (1) 
call pgrect $(7.5,11 ., 8.2,8.5)$

call pgsci(1) $6,8,3$, 'Hit <RETURN> or <ENTER> key')

call pgend

return

subroutine curssc

correlate screen cursor position with atom positions and display results character ch

character $\star 21$ st

integer pgcurs

character*6 'label, alabel

common /trfac/ xtrans, ytrans

common /ns/ npf, ndraw, norient, nvar

call pgsfs(1)

call pgsch(1.)

c *k* get cursor position

if (ch.eq. ' $x$ ' .or. ch.eq. ' $X$ ') return

if (ch.eq.' $d$ ' or. ch.eq. ' $D$ ') return

if (x.ge.10.4 and. $x .1 e .11$. and. $y . g e .8 .2$ and. $y .1 e .8 .5)$ return

if (2char(ch).eq.13)

$c \star \star \star$ initial values for variables

$x p t=x$

adiff $x=.0625$

adiffy $=.0625$

odiffx $=$ adiffx

atomid $=$,

alabel $=$.

iflag $=0$

nflag $=0$

rewind (npf)

$2 \operatorname{read}(\mathrm{npf}, 3$, end $=4$ ) label, tomid, $x \times x, y y$

format (11x, a6, 3x, a9, 4x, 2f8.0)

diffx $=$ abs (xx-xpt)

(diffx. le.adiffx .and. diffy.le.adiffy) nflag=nflag+1
(diffx.le.odiffx .and. diffy.le.odiffy) then

if (aiffx.le.adiff

atomid $=$ tomid

alabel = label

odiffy $=$ diffy

end if
go to 2

4 if (nflag.eq.0) write (str, 5

if (nflag.eg.1) write (str, 6) alabel, atomid
5 format ('Not near atom center')

format $(a 6,1 x, a 9,1+? ? \cdot)$

$c$ *** erase rectangle

call pgsci(0)

call pgsfs(1)

call pgrect $(0 ., 2.8,8,2,8.5)$

$c * * *$ redraw empty rectangle

call pgsci

call pgrect $(0 ., 2.8,8.2,8.5)$

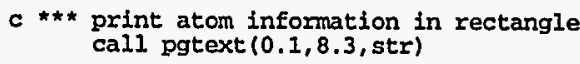

go to 1

end

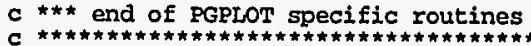

c $\star \star \star$ HPGL FILE OUTPUT

subroutine inithp

common /ns/ npf, ndraw, norient, nvax

character ESC

10 Write (Ename, 10)

$$
\text { open (unit=npf, file }=\text { fname, status= ' old' , er } x=12 \text { ) }
$$

11 continue

open (unit=npf, file= fname, status = ' new'

13 format $(/, \cdot$ ' HPGL file name: $\cdot, a)$

ESC=char (27)

write (npE, 21) ESC

write (npf,22)

if (norient. eq. 2) write (npf, 24)

21 format $\left(a 1, \cdot E^{\prime}\right)$

23 format ('IN;'/'SP1;'/'PW.15;')

format ('RO90., $;$ ')

return

subroutine colrhp(icolor)

common /ns/ npf, ndraw, norient, nvar

c *** in ORTEP icolor $=0 \Rightarrow$ black

plotter pen 1=black 
if (icol.eq.0) icol=1

21 format ('SP', il, $i$, ')

return
end

subroutine penwhp (penw)

rient, nvar

if (penw.eq.0.) then

penw $=.15$

penw=penw*.0252

end if

write (npf, 21) penw

, f5. $\left.2, i^{\prime}\right)$

return

subroutine penhp $(x, y$, ipen $)$

common ins/ npl, ndraw, norlent, nva

common /trfac/ xtrans, ytrans

$i x=\operatorname{nint}((x+x$ trans $) * 1000$.
$i y=\operatorname{nint}((y+y$ trans $) \cdot 1000$.

01 if (ipen.eg. 2) write (npf, 101) ix, iy

102 if (ipen,eq. 3) write (npf, 102) ix, iy

return

return

\section{subroutine endhp}

subroutine endhp

$\mathrm{SC}=\operatorname{char}(27)$

31 format ('PU;',1,'SPO;',1, 'PG;',1, 'IN; ')

write (MPE, 34) ESC

34 format (a1, $80 \mathrm{~A}$ )

write (npf, 35) ESC

close (npf)

return

end

c $\star \star \star \star$ end of HPGL Specific routines

\section{C}

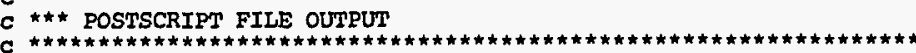

subroutine initps

common /ns/ npf ndraw, norient, nvar

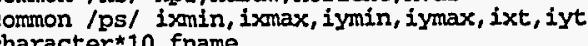

c *** initialize variables to calculate bounding box ixmin $=20000$

iymin $=20000$
iymax $=0$

do $11 i=1,999$

10 write (fname, 10) i

open (unit=npf, file=fname, status= ' old ' , err=12) close (npf)

11 continue

2 open (unit $=$ npf, file=fname, status=' new')

13 format $(\%, \cdot$ - Postscript file name: $\cdot$, a)

ixt $=0$
iyt $=0$

write (npf, 21)

write (npf,22)

write (npf, 23)

(norient.eg. 2) then

iyt=nvar

else

write (npf, 25)

end if

write (npf, 26)

write (npe, 27)

write (npf, 29)

write (npf,30)

write (npf, 31)

if (norient. eq. 2) write (npf, 33)

write (npf, 34)

1 format ( .7 ! PS-Adobe- 3.0 EPSF -3.0.$)$

format ( 1 \% 8 cree

format (' 8 \&BoundingBox: (atend)', $/$ ' $z$ \&pages : 1 ')

format (zzorientation: Landscape'

format ('szorientation: Portrait')

26 format (

(1) format ( 1 l (lineto) def:

format ' 'zzendprolog', /.'szpage: 11 ')

30 format (' 8zBeginPageSetup')

31 format ( 0.0720 .072 scale')

format (16,1x,16, translate'

format ( 90 rotate')

tlinecap 5 setlinewidth')

return

subroutine colrps(icolor) common /ns/ npf, ndraw, norient, nvar write (npf, 101)

101

if (icolor.eq.0) write (npf, 1)

if (icolor.eq.1) write (npf,1) 
if (icolor.eq.3) write (npf, 3

if

if (icolor.eq.6) write (npf, 6 )

if (icolor. eq. 7 ) wr

format (' 100 setrgbcolox')

format ( 010 setrgbcolor

format ( 01 setrgbcolor

format (' 1 1 1 setrgbcolor'

format (' 1 1 0 setrgbcolor')

return

end

subroutine penwps (penw)

npf, ndraw, norient, nvar

01 format ('stroke')

if (penw.eq. 0.) penw=5.

write (npf, 102) penw

102 format(f10.2,1x,' setlinewidth')

retur

subroutine penps $(x, y$, ipen $)$

common /trfac/ xtrans,ytrans

cormon /ns/ npf, ndraw, norient, nvar

common /ps/ ixmin, ixmax, iymin, iymax, ixt, iyt

$i x=\operatorname{nint}((x+x$ trans $) * 1000$.
$i y=\operatorname{nint}((y+y$ trans $) * 1000$.

if (ipen.eq. 2) write (npf, 101) ix, iy

101 format $\left.(i 6,1 x, i 6,1 x, 1)^{\prime}\right)$

102 format('stroke'/i6,1x,i6,1x, 'm')

$c * \star$ variables to calculate bounding box

if (ix. It.ixmin) ixmin=ix

fit.iymin) ixmax $=1 x$

if (iy.gt.iymax) iymax=iy

return

subroutine endps

common /ns/ npf, ndraw, norient, nvar common /ps/ ixmin, ixmax, iymin, iymax, ixt, iyt

write (npE, 25)

('stroke'/'shompage')

$c * \star \star$ calculate bounding box

if (norient.eq.1) then

iymn=Eloat (iymin+iyt)*.072 -

ixmx=float (ixmax+ixt) *.072 + ixmn=float $(i x t+i y \min ) * .072-2$

iymn=float $(i y t-i x \max ) * .072-2$

iymx=float $(i y t-i x \min ) * .072+2$

end if

if (ixmn. 1t. 0) ixmn=0

c *** put bounding box at end of postscript file

write (npf, 26) ixmn, iymn, ixmx, iymx

write (npf, 27)

27 format ('zzTrailer'/'zzEOF')

close (npf)

return

end

c $* \star *$ end of postscript specific routines

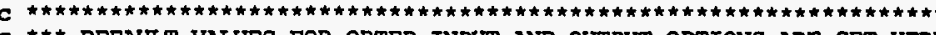
$c * * *$ DEFAULT VALUES FOR ORTEP INPUT AND OUTPUT OPTIONS ARE SET HERE

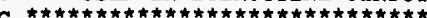

subroutine dflts

common /dfl/ infile, idraw, iorient, iout, ext, atomfi, fpaplen

character $\star 60$ infile, atomfj

$c \star \star \star$ name of default input file

$c * \star *$ where ortep drawing output should go
$c * \star *$ 1: Screen, 2: Postscript file, 3: HPGL file $1:$ sereen,

$c * * *$ orientation of drawing

1: portrait

$c \star * *$ height of page

fpaplen=11.

c *** where ortep text output should go

1: $f$ ill $=0$

$c * \star \star$ text output filename extension ext $=$ ' out

$c \star \star \star$ default name of external atom parameter file atomf $i=$ 'ATOMS.DAT'

return 


\section{INTERNAL DISTRIBUTION}

1. J. C. Bryan

2. G. J. Bunick

3-33. M. N. Burnett

34. P. D. Butler

35. B. C. Chakoumakos

36. P. Dai

37. J. A. Fernandez-Baca

38. A. A. Gakh

39. A. Habenschuss

40. W. A. Hamilton

41. J. B. Hayter

42. C. R. Hubbard

43-73. C. K. Johnson

74. S. A. McLuckey
75. H. A. Mook

76. S. E. Nagler

77. M. L. Poutsma

78. J. M. Ramsey

79. J. L. Robertson

80. S. Spooner

81. G. D. Wignall

82. M. Yethiraj

83. J. L. Zarestky

84. Central Research Library

85. Y-12 Technical Library

86. Laboratory Records - RC

87-686. ORTEP-III Document Distribution

\section{EXTERNAL DISTRIBUTION}

687-688. Office of Scientific and Technical Information, P.O. Box 62, Oak Ridge, TN 37831

689. B. R. Vincent, Molecular Structure Corporation, 3200 Research Forest Drive, The Woodlands, TX 77381

690. G. G. Johnson, Jr., Penn State University

691. A. Linden, Organic Chemistry Institute, University of Zurich, Winterthurerstrasse 190, CH-8057 Zurich, Switzerland

692. J. V. Silverton, NHLBI/LBC, 10/7N-307, 10 Center Dr MSC 1676, Bethesda, MD 20892-1676

693. M. Kroeker, Inst. f. Organ. Chemie, Univ. (TH) Darmstadt, Germany

694. W. Poll, Heinrich-Heine-Universitaet Duessseldorf, Institut fuer Anorganische Chemie und Strukturchemie, Universitaetsstrasse 1, D-40225 Duesseldorf, Germany

695. K A. Byriel, Department of Chemistry, The University of Queensland, Brisbane, QLD. 4072, Australia

696. B. Dalhus, Dept. of Chemistry, University of Oslo, PO Box 1033, Blindern, N0315 Oslo, Norway

697. Bill Harrison, Department of Chemistry, University of Western Australia, Nedlands, WA 6907, Australia

698. J. Bollinger, Department of Chemistry, Molecular Structure Center, Indiana University, Bloomington, IN 47405

699. J. C. Huffman, Department of Chemistry, Molecular Structure Center, Indiana University, Bloomington, IN 47405

700. K. M. Crennell, 'Fortran Friends', P.O.Box 64, Didcot, Oxon OX11 0TH 